\title{
2021 Scientific Session of the Society of American Gastrointestinal and Endoscopic Surgeons (SAGES), Las Vegas, Nevada, 31 August-3 September 2021: Podium Abstracts
}

(C) Springer Science+Business Media, LLC, part of Springer Nature 2021

S001

ROBOTIC VS. 'OPEN' MAJOR HEPATECTOMY: A PROPENSITY SCORE MATCHING STUDY

Iswanto Sucandy, MD, FACS; Kaitlyn Crespo, BS; Cameron Syblis, BS; Valerie Przetocki, BS; Timothy Bourdeau, BS; Sharona Ross, MD, FACS; Alexander Rosemurgy, MD, FACS; AdventHealth

Introduction: The data on major robotic hepatectomy are lacking. This study was undertaken to compare robotic vs. the 'open' major hepatectomy utilizing patient propensity score matching (PSM)

Methods: With IRB approval, we prospectively followed 183 patients who had undergone robotic or 'open' major hepatectomy, defined as the removal of three or more segments. 42 patients who underwent the 'open' approach were matched with 42 who underwent the robotic approach. The criteria for PSM was resection type, tumor size, cell type, and BMI. Survival was stratified for hepatocellular carcinoma (HCC), intrahepatic cholangiocarcinoma (IHCC), and colorectal liver metastases (CLM). The data are presented as median(mean SD).

Results: Operative duration for the robotic approach was $293(302 \pm 131.5)$ vs. $280(300 \pm 115.6)$ minutes for the 'open' approach $(\mathrm{p}=\mathrm{NS})$ (Table); estimated blood loss (EBL) was $200(239 \pm 183.6)$ vs. $300(491 \pm 577.1) \mathrm{mL}(\mathrm{p}=0.01)$. There were zero postoperative complications with a Clavien-Dindo classification $\geq$ III for the robotic approach and three for the 'open' approach $(\mathrm{p}=\mathrm{NS})$. ICU length of stay $(\operatorname{LOS})$ was $1(1 \pm 0)$ vs. $2(3 \pm 2.0)$ days $(\mathrm{p}=0.0001)$ and overall LOS was $4(4 \pm 3.3)$ vs. $6(6 \pm 2.7)$ days $(\mathrm{p}=0.003)$. When utilizing the robotic approach, patients with malignant tumor pathology (i.e., HCC, IHCC, and CLM) lived significantly longer (p $=0.05$ ). Patients with the robotic approach for HCC pathology lived significantly longer, as well $(\mathrm{p}=0.05)$

Conclusion: Utilizing propensity matched patients, the robotic approach led to lower EBL, shorter ICU LOS, and shorter overall LOS while maintaining similar operative duration and promoting survival. We believe that the robotic approach is safe and efficacious and should be the preferred approach for major hepatectomy.

\begin{tabular}{|c|c|c|c|}
\hline \multicolumn{4}{|c|}{ Robotic vs 'Open’ After Propensity Score Matching for Major Hepatectomy } \\
\hline & 'Open' & Robotic & Total/p-value \\
\hline Demographic data & $\mathrm{n}=42$ & $\mathrm{n}=42$ & $\mathrm{n}=84$ \\
\hline Age(years) & $64(64 \pm 12.1)$ & $61(61 \pm 12.5)$ & $\mathrm{p}=\mathrm{NS}$ \\
\hline $\operatorname{Sex}(M / W)$ & $19 \mathrm{M} / 23 \mathrm{~W}$ & $24 \mathrm{M} / 18 \mathrm{~W}$ & $\mathrm{p}=\mathrm{NS}$ \\
\hline BMI $\left(\mathrm{kg} / \mathrm{m}^{2}\right)$ & $27(27 \pm 4.6)$ & $28(28 \pm 5.9)$ & $\mathrm{p}=\mathrm{NS}$ \\
\hline \multicolumn{4}{|l|}{ Perioperative variables } \\
\hline Operative duration(min) & $280(300 \pm 115.6)$ & $293(302 \pm 131.5)$ & $\mathrm{p}=\mathrm{NS}$ \\
\hline Size of lesion $(\mathrm{cm})$ & $7(7 \pm 4.0)$ & $6(6 \pm 3.8)$ & $\mathrm{p}=\mathrm{NS}$ \\
\hline Pathology (HCC $/ \mathrm{HCC} / \mathrm{CLM} / \mathrm{O}$ ther) & $13 / 12 / 6 / 11$ & $13 / 12 / 6 / 11$ & $\mathrm{p}=\mathrm{NS}$ \\
\hline Distance to $\operatorname{margin}(\mathrm{cm})$ & $1(1 \pm 0.9)$ & $1(1 \pm 1.3)$ & $\mathrm{p}=\mathrm{NS}$ \\
\hline Margin status(R0/R1/R2) & $31 / 4 / 0$ & $29 / 6 / 0$ & $\mathrm{p}=\mathrm{NS}$ \\
\hline Estimated blood loss $(\mathrm{mL})$ & $300(491 \pm 577.1)$ & $200(239 \pm 183.6)$ & $\mathrm{p}=0.01^{*}$ \\
\hline Postoperative complications(n) & $\begin{array}{l}\text { UTI(2), Anastomotic } \\
\text { leak(1), Sepsis }(1), \\
\text { Respiratory failure(2), } \\
\text { Systemic inflammatory } \\
\text { response syndrome(1) }\end{array}$ & $\operatorname{Ileus(2)}$ & $\mathrm{p}=\mathrm{NS}$ \\
\hline Clavien-Dindo classification $\geq I I(n)$ & IVa(1), V(3) & None & $\mathrm{p}=\mathrm{NS}$ \\
\hline Length of ICU stay(days) & $2(3 \pm 2.0)$ & $1(1 \pm 0)$ & $\mathrm{p}=0.0001^{*}$ \\
\hline Length of stay(days) & $6(6 \pm 2.7)$ & $4(4 \pm 3.3)$ & $\mathrm{p}=0.003^{*}$ \\
\hline 30-day readmission(n) & 11 & 13 & $\mathrm{p}=\mathrm{NS}$ \\
\hline In-hospital mortality(n) & 3 & 1 & $\mathrm{p}=\mathrm{NS}$ \\
\hline
\end{tabular}

Ns (not significant)

+significance is defined as p-valueso.05 
S002

\section{Liver Regeneration Response after Open and Laparoscopic Left Partial Hepatectomy in an Experimental Porcine Model, Interpreted considering timing}

Constantinos S Mammas, MD, MSc, PhD; Nick Kavantzas, MD, PhD; Spyros Dourakis, MD, PhD; Surgical Laboratory Constantinos Tountas.

Objectives: Liver regeneration in the remnant liver of a porcine experimental model after Open (OLPH) and Laparoscopic Left Partial Hepatectomy (LLPH) is compared.

Materials and Methods: Two pairs of double-series of twenty-nine liver tissues taken from twenty nine porcine livers, after OLPH or LLPH (PD0) and on the 7th postoperative day (PD7), were randomly allocated into two groups: the OLPH $(\mathrm{n} 1=19 \times 2)$ and the LLPH $(\mathrm{n} 2=10 \times 2)$.The liver tissue sections in each group, had been prepared and stained with PCNA, Gaspase-III and anti-Ubiquitin and anti-VEGF stain. A PCNA based proliferative index (LCPI), a Gaspase-III based (Liver Cell Apoptotic Index (LCAI), and an Ubiquitin based intracellular stress index (LISI) were prospectively estimated by microscopic visualization of the aforementioned tissue stained samples. A VEGF based angiogenesis index (LCANGI) was estimated by microscopic visualization (MV) of a sample of twenty eight tissue sections $(\mathrm{n} 1=8 \times 2, \mathrm{n} 2=6 \times 2)$ retrospectively. A PASstain Candida Species RL lobule colonization index (CRLLCI) of a sample of twenty eight tissue sections $(\mathrm{n} 1=7 \times 2, \mathrm{n} 2=7 \times 2)$ estimated by MV. LCPI, LCAI, LISI, LCANGI and CRLLCI compared between OLPH and LLPH on PD0 and on PD7.

Results: There was not any difference in the mean weights of the remnant livers and in the post-surgical complications rates between OLPH and LLPH on PD7. Statistical comparisons of LISI resulted in a statistically significant difference, favoring the OLPH group on PD0 $(p<0.1)$. LCPI was statistically significantly higher after OLPH than LLPH on PD7 $(p=0.002)$. LCANGI OLPH was statistically significantly higher than LCANGILLPH, on PD7 $(p=0.028)$. No difference was found in the apoptotic level between OLPH and LLPH on PD0 (0.926) and on PD7 ( $p>$ $0.313)$. CRLLCI was significantly higher in the OLPH in comparison to the LLPH on PD0 $(\mathrm{p}=0.158)$ an on PD7 $(\mathrm{p}=0.006)$.

Conclusions: Given the lack of difference in the apoptotic level between groups on PD0 and on PD7, increased cellular stress after OLPH and the significantly increased liver proliferation and angiogenesis in the remnant liver after OLPH than after LLPH on PD7, it seems that the regenerative process after OLPH is delayed, while liver regeneration after LLPH on PD7 has been processed according to the expected time depended liver cell proliferation phases. This means that LLPH under indications and safe surgery in experienced hands is indicated, it is safer than OLPH and can have a relatively optimized and on time liver regeneration response, especially for oncologic patients suffering from viral (HBV, HCV, HIV, Covid-19 etc.) and other, crucial for post-PHx liver regeneration process, co-existed diseases.

\section{S003}

\section{Improved Outcomes of Laparoscopic Liver Resection} for Hepatocellular Carcinoma Located in Posterosuperior Segments of the Liver

Yujin Kwon ${ }^{1}$; Jai Young $\mathrm{Cho}^{2}$; Ho-Seong $\mathrm{Han}^{2}$; Yoo-Seok Yoon ${ }^{2}$; Hae Won $\mathrm{Lee}^{2}$; Jun Suh Lee ${ }^{2}$; Boram Lee ${ }^{2}$; Moonwhan Kim ${ }^{2} ;{ }^{1}$ Department of Surgery, Seoul Medical Center; ${ }^{2}$ Department of Surgery, Seoul National University Bundang Hospital.

Background: LLR is widely adapted for HCC, while LLR in PS segments is still challenging. With recent improvement of techniques and accumulation of experiences, LLR in PS segments is feasible, but studies investigating the result after the modifications are lacking.

Methods: In this single center, retrospective study, 149 patients who underwent LLR for HCC located in PS segments from September 2003 to December 2016 were analyzed. The patients were divided into Group $1(n=43)$ and Group $2(n=106)$ who underwent LLR before and after 2012 respectively, when advanced techniques including use of intercostal trocars, Pringle maneuver, and semi-lateral position of patient were introduced. Also, these patients were compared with those who underwent open liver resection (OLR; $n=124)$ for HCC in PS segments during the same period.

Results: Mean operative time ( $394.7 \mathrm{~min}$ vs $331.2 \mathrm{~min} ; \mathrm{P}=0.013$ ), intraoperative blood loss ( $1545.8 \mathrm{ml}$ vs $1208.2 \mathrm{ml} ; \mathrm{P}=0.020$ ), and hospital stay (11.6 days vs 9.2, $\mathrm{P}<0.001)$ were significantly less in Group 2. Postoperative complication rate ( $18.6 \%$ vs $18.9 \% ; \mathrm{P}=0.970)$, open conversion rate $(23 \%$ vs $17 \% ; \mathrm{P}=0.374)$, 5 -year overall $(79 \%$ vs $89 \% ; \mathrm{P}=0.607)$ and 5 -year disease-free $(52 \%$ vs $53 \% ; \mathrm{P}=0.657)$ survival rates were not significantly different between the groups. Compared to the OLR group, complication rate $(40.3 \%$ vs $18.8 \%$; $\mathrm{P}<0.001)$ and hospital stay (17.6 days vs 9.7 days; $\mathrm{P}<0.001$ ) were significantly lower in the LLR group. Conclusion: The complexity of LLR for HCC in PS segments is being gradually overcome by the introduction of advanced techniques.

\section{S004}

\section{ANALYSIS OF SURGICAL APPROACH AND TUMOR DISTANCE TO MARGIN AFTER LIVER RESECTION FOR COLORECTAL LIVER METASTASES}

Iswanto Sucandy, MD, FACS; Emanuel Shapera, MD; Kaitlyn Crespo, BS Cameron Syblis, BS; Timothy Bourdeau, BS; Valerie Przetocki, BS; Sharona Ross, MD, FACS; Alexander Rosemurgy, MD, FACS; AdventHealth Tampa.

Introduction: This study was undertaken to compare tumor distance to margin after robotic vs. 'open' hepatectomy for colorectal liver metastases (CLM) and to determine the effect of preoperative variables on tumor distance to margin and determine the effect of tumor distance to margin on postoperative variables and survival. Methods: With IRB approval, we prospectively followed 56 patients who underwent a robotic or 'open' hepatectomy for treatment of CLM. The relationships between the tumor distance to margin and perioperative data were determined using regression analyses. For illustrative purposes, and by convention, tumor distance to margin is presented in three cohorts, $\leq 1 \mathrm{~mm}, 1.1-9.9 \mathrm{~mm}$, and $\geq 10 \mathrm{~mm}$, and then further stratified by approach (robotic or 'open'). Survival was determined for tumor distance to margin and operative approach. The data are presented as median(mean $\pm \mathrm{SD})$.

Results: There was a significant difference in the frequency of tumor distance to margin for patients who underwent a robotic vs. 'open' hepatectomy; $\leq 1 \mathrm{~mm}(17 \%$ vs. $50 \%), 1.1-9.9 \mathrm{~mm}(52 \%$ vs. $29 \%)$, and $\geq 10 \mathrm{~mm}(31 \%$ vs. $7 \%)(\mathrm{p}=0.04)$. The robotic vs. 'open' approach had an operative duration of $375(358 \pm 130.5)$ vs. $269(279 \pm 113.3)$ minutes $(\mathrm{p}=0.05)$, ICU length of stay (LOS) of $0(0 \pm 0.8)$ vs. $0(1$ $\pm 2.0)$ days $(\mathrm{p}=0.01)$, and hospital LOS of $4(5 \pm 2.6)$ vs. $7(7 \pm 4.0)$ days $(\mathrm{p}=0.04)$. There was a longer ICU LOS in patients who had a tumor distance to margin of $\leq$ $1 \mathrm{~mm}[0(1 \pm 2.0)]$ vs. $1.1-9.9 \mathrm{~mm}[0(0 \pm 1.0)]$ and $\geq 10 \mathrm{~mm}[0(0 \pm 0.2)$ days $](\mathrm{p}=$ 0.03 for each). Patients with a tumor distance to margin of $\leq 1 \mathrm{~mm}$ and $1.1-9.9 \mathrm{~mm}$ had a median survival of 49 months and 24 months, respectively. Median survival for patients with tumor distance to margin of $\geq 10 \mathrm{~mm}$ has not been reached but is $>$ 84 months.

Conclusions: The use of the robotic approach leads to greater tumor distance to margin as well as shorter ICU and hospital LOS, but with longer operations. Shortest tumor distance to margin led to longer ICU LOS. The robotic approach produces better short-term outcomes and greater tumor distance to margin, but the latter has an inconsistent effect on survival.

\begin{tabular}{|l|c|c|c|}
\hline \multicolumn{4}{|c|}{ Robotic vs. 'Open' Colorectal Liver Metastases } \\
\hline & Robotic & 'Open' & $\mathrm{p}$-values \\
\hline Sex(M/W) & $\mathrm{n}=42$ & $\mathrm{n}=14$ & \\
\hline Age(years) & $26 \mathrm{M} / 16 \mathrm{~W}$ & $6 \mathrm{M} / 8 \mathrm{~W}$ & $\mathrm{p}=0.23$ \\
\hline BMI(kg/m²) & $63(61 \pm 13.5)$ & $72(69 \pm 12.3)$ & $\mathrm{p}=0.06$ \\
\hline Size of Lesion(cm) & $28(28 \pm 5.7)$ & $25(27 \pm 6.3)$ & $\mathrm{p}=0.58$ \\
\hline Estimated Blood Loss(mL) & $200(265 \pm 303.8)$ & $200(372 \pm 629.1)$ & $\mathrm{p}=0.40$ \\
\hline Clavien-Dindo Complications(n) & $\mathrm{II}(4), \mathrm{IVa}(1)$ & $\mathrm{II}(1), \mathrm{III}(1), \mathrm{V}(1)$ & $\mathrm{p}=0.15$ \\
\hline 30-Day Readmission(n) & 6 & 2 & $\mathrm{p}=1.00$ \\
\hline In-Hospital Mortality(n) & 0 & 1 & $\mathrm{p}=1.00$ \\
\hline 90-Day Mortality(n) & 2 & 1 & $\mathrm{p}=1.00$ \\
\hline
\end{tabular}


S005

\section{MINIMALLY INVASIVE LIVER RESECTION FOR INTRAHEPATIC CHOLANGIOCARCINOMA-A WORD OF CAUTION}

Omid Salehi, MD; Vera Kazakova, MD; Eduardo A Vega, MD; Onur C Kutlu, MD Sylvia V Alarcon, MD; Claudius Conrad, MD; St Elizabeth's Medical Center.

INTRODUCTION: While minimally invasive liver resection (MILR) vs. open approach (OLR) for various tumor types has been shown to be safe, the perioperative and oncologic safety of MILR for intrahepatic cholangiocarcinoma (ICC) specifically remains ill-defined.

METHODS AND PROCEDURES: The National Cancer Database (NCDB) was queried for patients with ICC undergoing liver resection from 2010-2015. 1:1 Propensity Score Matching (PSM) was used to control for confounders. Binary and logistic regression was applied to compare short-term outcomes. Kaplan-Meier survival analyses were performed to evaluate median survival. Cox multivariable regression was used identify factors associated with survival.

RESULTS: 988 patients matched inclusion criteria: 140 (14.2\%) MILR and 848 (85.8\%) OLR resulting in 115 patients MILR and OLR after 1:1 PSM with c-index of 0.733. MILR had lower unplanned 30-day readmission [OR 0.075, P $=0.014 ; 0.9$ vs $10.4 \%, \mathrm{P}=0.002$ ] and positive margin rates [OR $0.361, \mathrm{P}=0.011 ; 8.7$ vs $20.9 \%$, $\mathrm{P}=0.009$ ] and shorter hospital length of stay (LOS) [OR 0.941, $\mathrm{P}=0.026 ; 6.2 \mathrm{vs} 9.2$ mean days, $\mathrm{P}=0.006]$, but worse lymph node yield [1.52 vs 2.07 mean lymph nodes examined, $\mathrm{P}=0.001]$. No difference was found for 30-day mortality [OR 1.697, $\mathrm{P}=$ $0.476 ; 4.4$ vs $2.6 \%, \mathrm{P}=0.472$ ] and 90 -day mortality [OR $1.426, \mathrm{P}=0.555 ; 6.1$ vs $4.4 \%, \mathrm{P}=0.553$ ]. Receipt of chemotherapy [OR $1.147, \mathrm{P}=0.651 ; 27$ vs $24.4 \%, \mathrm{P}=$ 0.651 ], and specifically adjuvant chemotherapy [OR $0.750, \mathrm{P}=0.399 ; 16.5$ vs $20.9 \%, P=0.398]$, was not affected by surgical approach. Nevertheless, multivariate analysis revealed that MILR was associated with poorer overall survival compared to OLR [HR 2.454, 95\% CI 1.422-4.235, P = 0.001; median OS 40.9 vs 63.8 months, $\mathrm{P}$ $=0.036]$; other factors associated with worse survival included advanced clinical stage, tumor size, length of hospital stay, and positive margins. However, approach did not influence survival when comparing non-PSM MILR and OLR cohorts; subgroup analysis of the PSM cohort revealed that survival differences from approach were dependent on major hepatectomy, tumor size $>4 \mathrm{~cm}$, or negative margins. Additionally, when analyzed separately, robotic approach had comparable survival with OLR, whereas laparoscopic approach continued to fare worse.

CONCLUSION: MILR vs. OLR, in spite of providing improved resection margins, shorter hospital LOS, and reduced unplanned readmission rate, contributes to poorer lymph node yield and therefore understaging, and ultimately worse survival. Hence, ICC should only be approached via MILR if the same quality of oncologic surgery (especially lymphadenectomy) and survival of OLR can be achieved and may be currently best used for minor hepatectomies and smaller tumors.

\section{S006}

The Initiation, Standardization and Proficiency (ISP) Phases of the Learning Curve for Minimally Invasive Liver Resection: comparison of a Fellowship-trained Surgeon with the Pioneers and Early Adopters

Andrew A Gumbs, MD ${ }^{1}$; Mohamed Abu Hilal, $\mathrm{MD}^{2}$; Roland Croner, $\mathrm{MD}^{3}$; Michel Gagner, MD ${ }^{4}$; Brice Gayet ${ }^{5}$; Elie Chouillard, $\mathrm{MD}^{1}$; ${ }^{1}$ Centre Hospitalier Intercommunal de Poissy/Saint-Germain-en-Laye; ${ }^{2}$ Fondazione Poliambulanza Istituto Ospedaliero; ${ }^{3}$ University of Magdeburg; ${ }^{4}$ Hôpital du Sacre Coeur; ${ }^{5}$ Institut Mutualiste Montsouris.

Background: Using the Ideal Development Exploration Assessment and Long-term study (IDEAL) paradigm, Halls et al. created risk-adjusted cumulative sum (RACUSUM) curves concluding that Early Adopters (EA) of minimally invasive (MI) liver resection obtained similar results after fewer cases when compared to Pioneers (P). In this study, we applied this framework to a MI Hepatic-Pancreatic and Biliary fellowship-trained surgeon (FT) in order to assess where along the curves this generation fell.

Methods: The term FT was used to designate surgeons without previous independent operative experience who went from residency directly into fellowship. Three phases of the learning curve were defined using published data on EAs and Ps of M Hepatectomy, including phase 1 (initiation) (i.e. the first 17 or 50), phase 2 (standardization) (i.e. cases $18-46$ or $1-50$ ) and phase 3 (proficiency)(i.e. cases after 46 , 50 or 135). Data analysis was performed using the Social Science Statistics software. Statistical significance was $\mathrm{p}<0.05$.

Results: From November 2007 until April 2018, 95 MI hepatectomies were performed by a FT. During phase 1, the FT approached larger tumors than the EA group $(\mathrm{p}=0.002)$, that were more often malignant $(94.1 \%)$ when compared to the $\mathrm{P}$ group $(52.5 \%)(\mathrm{p}<0.001)$. During phase 2 the FT operated on larger tumors and more malignancies $(93.1 \%)$ when compared to the Ps $(p=0.004$ and $p=0.017$, respectively). In the phase 3 , the EAs tended to perform more major hepatectomies (58.7) when compared to either the FT $(30.6 \%)(\mathrm{p}=0.002)$ or the P's cases $51-135$ and after 135 (35.3\% and 44.3\%, respectively) (both p-values $<0.001)$. When compared to the Ps cases from 51-135, the FT operated on more malignancies $(p=0.012)$, but this was no longer the case after 135 cases by the Ps $(p=0.164)$. There were no statistically significant differences when conversions, major complications or 30 and 90-day mortality were compared among these 3 groups.

Discussion: Using the IDEAL framework and RA-CUSUM curves, a FT surgeon was found to have curves similar to EAs despite having no previous independent experience operating on the liver. As in our study, FTs may tend to approach larger and more malignant tumors and do more concomitant procedures in patients with higher ASA classifications than either of their predecessors, without statistically significant increases in morbidity or mortality. It is possible that the ISP (i.e., initiation, standardization, proficiency) model could apply to other innovative operations, creating different learning curves depending on where along the IDEAL paradigm surgeons fall 
S008

\section{SUBJECTIVE VS OBJECTIVE ASSESSMENT OF SIMULATION PERFORMANCE ON LAPAROSCOPIC CHOLECYSTECTOMY: ARE WE EVALUATING THE RIGHT THINGS?}

Yohei Kojima, MD, $\mathrm{PhD}^{1}$; Harry J Wong, $\mathrm{MD}^{2}$; Kristine Kuchta, $\mathrm{MS}^{3}$; Stephen Haggerty, MD, FACS ${ }^{3}$; Woody Denham, MD, FACS ${ }^{3}$; John Linn, MD, FACS 3 ; Michael Ujiki, MD, FACS ${ }^{3} ;{ }^{1}$ Northshore University HealthSystem; Kyorin University Hospital; ${ }^{2}$ Northshore University HealthSystem; University of Chicago Medicine; ${ }^{3}$ Northshore University HealthSystem.

Background: Simulation has become a vital tool in surgical training, with virtual reality (VR) surgical simulators meeting the trainees' need for repeated practice without impacting patient care. While assessment of simulation performance has traditionally relied on expert surgeon feedback, VR laparoscopic simulators can provide immediate objective performance assessment without observer evaluation. This study aims to explore the association between subjective observer evaluation and VR laparoscopic simulator performance metrics in a laparoscopic cholecystectomy (LC) simulation module.

Methods: A LC simulation module using a VR laparoscopic simulator was completed by PGY2-3 general surgery residents during a two-week skills curriculum at a single institution consisting of pre-tests, mentoring sessions with an attending, deliberate practice, and post-tests. Simulation performance was recorded and evaluated by a trained evaluator using the validated Global Operative Assessment of Laparoscopic Skills (GOALS) form, the Objective Structured Assessment of Technical Skills (OSATS) form, and a LC-specific simulation assessment form (LCSIM). Objective performance metrics were also obtained from the VR laparoscopic simulator system. Performance before the curriculum (pre-test) and after the curriculum (post-test) were compared using the Wilcoxon signed-rank test. Spearman correlation coefficients (r) were used to compare evaluator assessment to objective simulator metrics.

Results: Fourteen residents who completed the simulation module also had objective simulator performance metrics available. There were significant improvements from pre-test to post-test on each component of GOALS, OSATS, and LC-SIM scores (all $p$ values $<0.05$ ). In terms of objective simulator assessment, significant improvements were noted in time to extract gallbladder $(481 \pm 221$ vs $909 \pm 366 \mathrm{~s}, \mathrm{p}=$ $0.019)$, total number of movements $(475 \pm 264$ vs $839 \pm 324, \mathrm{p}=0.012)$, and total path length $(955 \pm 475$ vs $1775 \pm 632 \mathrm{~cm}, \mathrm{p}=0.012)$ when comparing post-test to pre-test. While number of movements and total path lengths of both hands decreased, speed of right instrument also decreased from $4.1+2.7$ to $3.0 \pm 0.7 \mathrm{~cm} / \mathrm{sec}(\mathrm{p}=$ 0.007). Average speed of left instrument was associated with scores for respect for tissue $(\mathrm{r}=0.60, \mathrm{p}<0.05)$ and depth perception $(\mathrm{r}=0.68, \mathrm{p}<0.05)$ on post-test evaluations. Otherwise, few correlations were identified between subjective and objective measures of simulation performance.

Conclusions: Our study demonstrated significant improvement in procedure-specific technical skills based on observer assessment, as well as several simulator metrics after simulation. However, fewer than expected correlations were identified between the subjective evaluator and objective simulator assessments, suggesting the two evaluation modalities were measuring different aspects of the technical skills. Therefore, more investigation is needed to assess the utility of simulator metrics in skills training.

\section{S009}

SAGES Safe Cholecystectomy Modules Improve Surgeons' Abilities in Important Domains: A Randomized Controlled Trial among Surgeons in Practice

Joshua J Weis, $\mathrm{MD}^{1}$; L M Brunt, $\mathrm{MD}^{2}$; Madhuri Nagaraj, $\mathrm{MD}^{1}$; Amin Madani, MD, $\mathrm{PhD}^{3}$; Horacio Asbun, $\mathrm{MD}^{4}$; Brian Davis, MD ${ }^{5}$; Sharmila Dissanaike, $\mathrm{MD}^{6}$; Michael Ujiki, $\mathrm{MD}^{7}$; Carl J Westcott, $\mathrm{MD}^{8}$; Adnan Alseidi, MD, EdM ${ }^{9} ;{ }^{1}$ University of Texas Southwestern; ${ }^{2}$ Washington University in St. Louis; ${ }^{3}$ Colombia University; ${ }^{4}$ Baptist Health South Florida; ${ }^{5}$ Texas Tech University Health Science Center El Paso; ${ }^{6-}$ Texas Tech University Health Science Center; ${ }^{7}$ NorthShore University Health System; ${ }^{8}$ Wake Forest Baptist Medical Center; ${ }^{9}$ University of California San Francisco.

Background: Despite advantages of laparoscopic cholecystectomy, rates of major bile duct injury (BDI) during laparoscopic cholecystectomy remain unacceptably high relative to historical data on open cholecystectomy. SAGES is a leader in advocating for a universal culture of safety during cholecystectomy, and in October 2018, SAGES released the Safe Cholecystectomy Modules, which define specific strategies to minimize the risk of BDI. This study aims to investigate whether this curriculum can change the application of safe strategies during laparoscopic cholecystectomy.

Methods: Practicing surgeons were recruited from the membership of SAGES as well as the American College of Surgeons Advisory Council for Rural Surgery. All participants completed a dynamic, objective baseline assessment (pre-test) that offered a menu of possible paths to proceed through virtual scenarios. Based on the actions selected, the assessment would offer a new menu of actions until a scenario endpoint was reached. The scenarios involved interpreting cholangiograms, troubleshooting difficult cases, and managing BDI. Participants' dissection strategies during cholecystectomy were also compared to the strategies of a panel of 15 experts based on scores on the Think Like a Surgeon validated web-based platform. Participants were then randomized to complete the Safe Cholecystectomy modules (Safe Chole participants) or to participate in usually scheduled CME activities (controls). Both groups completed repeat assessments (post-tests) one month after randomization. The primary outcomes were the frequencies of errors during interpretation of cholangiograms, management of BDI, troubleshooting difficult dissections, and identification of adequate critical views of safety. Chi square analyses were used to analyze differences in error frequency between groups. Scores on the dissection assessment were compared with independent samples t-tests.

Results: Overall, 41 participants were eligible for analysis including 18 Safe Chole participants and 23 controls. The two groups had no significant differences in pre-test scores. At post-test, Safe Chole participants made significantly fewer errors managing BDI and interpreting cholangiograms (Figure). However, Safe Chole participants displayed a similar frequency of errors when evaluating adequate critical views of safety. When facing questions about challenging dissections, Safe Chole participants were more likely to use laparoscopic bailout maneuvers on the post-test compared to controls $(80 \%$ vs. $58 \%$ respectively, $\mathrm{p}=0.03)$. Dissection strategies were not significantly different between groups on the pre-test or post-test.

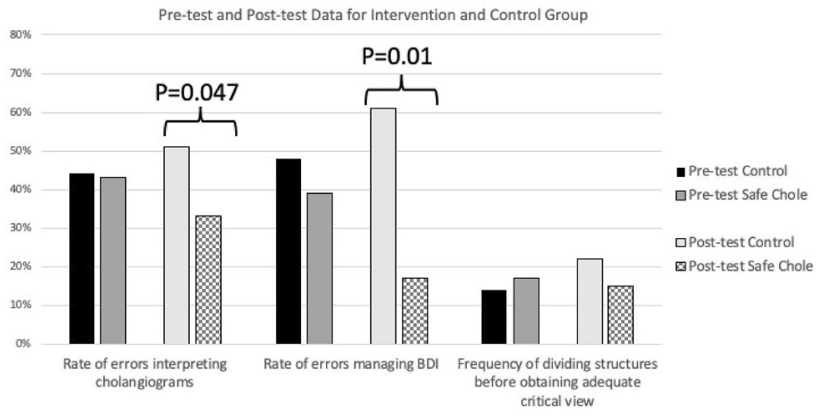

Conclusions: In this randomized-controlled trial, the SAGES Safe Cholecystectomy modules improved surgeons' abilities to interpret cholangiograms and safely manage BDI. Additionally, surgeons who studied the modules were more likely to utilize laparoscopic bailout maneuvers compared to controls. 
S010

\section{Certification of practical skills: A feasible and scalable methodology to assess practical skills objectively through remote technology.}

Francisca Belmar, $\mathrm{MD}^{1}$; Ivan Paul, $\mathrm{MD}^{1}$; Catalina Ortiz, $\mathrm{MD}^{1}$; Francisco Molina, $\overline{\mathrm{MD}}^{1}$; Javier Vela, $\mathrm{MD}^{1}$; Caterina Contreras, $\mathrm{MD}^{1}$; Adnan Alseidi, $\mathrm{MD}^{2}$; Gabriel Escalona, MD ${ }^{1}$; Pablo Achurra, $\mathrm{MD}^{1}$; Julian Varas, $\mathrm{MD}^{1}$; ${ }^{1}$ Pontificia Universidad Católica de Chile; ${ }^{2}$ University of California, San Francisco.

INTRODUCTION: Certification is required in most countries to perform as a specialist. In the USA and Chile, surgeons' certification consists of two theoretical exams, written and oral, without an objective measure of surgical competency. Since surgical skills are crucial to perform as a surgeon, their assessment should be included in any board certification. However, to develop and implement an exam that allows measuring the technical level of performance is challenging, especially nationwide. We aimed to design and validate a large-scale examination to certificate surgical skills using simulation models and a digital platform for blind, remote, and asynchronous evaluation.

METHODS: Surgeons completing their title-revalidation process in Chile underwent a previously validated eight-station OSCE with simulated procedures. The stations were designed to discriminate competency and had different levels of difficulty: (1)Intestinal anastomosis; (2)Laparoscopic basic/advanced station; (3)Cardiac injury; (4)Vascular anastomosis; (5)Bowel ostomy confection; (6)Lung injury; (7)Surgeon's Knot-tying and basic suturing; (8)Recess. All procedures were recorded in two complementary angles and uploaded to a digital platform for videobased assessment. Two expert surgeons blindly and asynchronously evaluated the procedures remotely. For the evaluation process OSATS global rating scale, specific rating scales, end-product quality, and procedural time were measured through the digital platform. The outcomes were weighted according to which were more fundamental to surgical practice. The cutoff-score was set at $75 \%$. Time, costs and the need for human resources for the examination were registered. A seven-item questionnaire to measure participants' perception and debriefing was conducted after the OSCE.

RESULTS: Sixty-eight participants were evaluated in 9 examinations; $18(26 \%)$ were women and $43(63 \%)$ over 40 -years-old. A total of 476-procedures were performed and 816 high-resolution videos were recorded and uploaded to the digital platform. Seven non-professional helpers assisted the participants, mounted and unmounted each station. Examination lasted $220 \mathrm{~min}(\mathrm{r} 180-240)$. The surgical expert training for online evaluation and platform usage lasted $30 \mathrm{~min}$. The average online evaluation time for each procedure was 5-8 $\mathrm{min}$ and 56 min per participant. Sixtythree $(91 \%)$ passed the exam, with an average score of $82 \%(68-91 \%)$.The participants rated the overall organization and execution of the examination as very good. Execution and materials cost were $\$ 325 /$ student.

CONCLUSION: In a brief period of time a considerable number of specialists were objectively evaluated.The use of asynchronous and remote evaluation through a digital platform allows a cost-effective assessment of surgical skills easy scaleup.

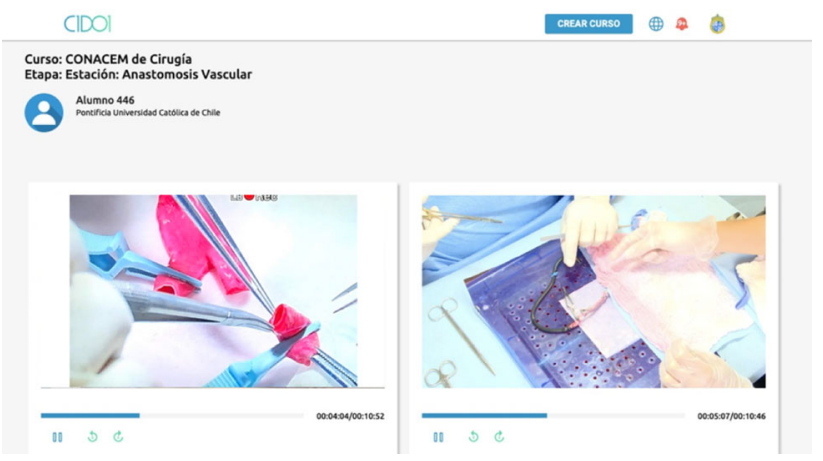

S011

\section{What does the operative team think of intraoperative telecoaching among surgeon peers?}

Golsa Shafa, BSc ${ }^{1}$; Caterina Masino, $\mathrm{MA}^{2}$; Amin Madani, MD, PhD, $\mathrm{FRCSC}^{3}$; Allan Okrainec, MD, MHPE, FRCSC, FACS ${ }^{3} ;{ }^{1}$ Temerty Faculty of Medicine, University of Toronto, Toronto, ON, Canada; ${ }^{2}$ Temerty Advanced Surgical Education and Simulation Centre, University Health Network, Toronto, ON, Canada; ${ }^{3}$ Division of General Surgery, University Health Network, Toronto, ON, Canada.

INTRODUCTION: Telecoaching, intraoperative coaching through the use of virtual presence, has been suggested as a tool to overcome logistical barriers with inperson coaching. However, telecoaching has its own set of challenges that requires further understanding to inform the design of future programs. This qualitative study explores the perceptions of the multidisciplinary operative team of a surgical telecoaching program.

METHODS AND PROCEDURES: A telecoaching program was implemented at a multi-site academic institution using the Visitorl ${ }^{\circledR}$ remote presence system (Karl Storz, Germany). A convenience sample of operative team members present during telecoaching sessions underwent semi-structured interviews. Interviews were recorded, transcribed verbatim, and thematically analyzed by two independent reviewers in an iterative fashion until data reached saturation.

RESULTS: Twelve team members who participated in two telecoaching events were interviewed, including 4 surgeons, 2 surgical fellows, 2 surgical residents, 1 anesthesiologist, 1 anesthesiology fellow and 2 nurses. The following four central themes emerged from the interview data: effective communication and collaboration, acquiring new knowledge and skills, positive regard for surgeon mentees, absence of workflow disruption. The surgeon mentees reported that the telecoaching session met their expectations and learning goals. Coaches also reported learning from the sessions. Barriers to greater adoption included scheduling and finding the appropriate patient cases for coaching. In addition, surgeon mentees expressed concerns about their colleagues' and patients' perception of their autonomy and expertise when requesting to have a coach. Conversely, team members unanimously reported a positive impression of surgeon mentees for taking additional measures to improve their performance and patient outcomes. Establishing robust privacy standards and consent pathways for both patients and staff were of paramoun importance for the successful integration of this technology. Nurses and anesthesiologists expressed concerns with space constraints, interruptions with workflow and internet connectivity as issues that need to be addressed and recommended coordination with the operative team before the session. Residents and fellows felt telecoaching was innovative, conducive to their own learning, and relevant for complex cases, despite a potential for taking away from residents' operative experience.

CONCLUSION: Telecoaching was viewed very favorably and practical considerations were identified for its continued use in an operating room environment. The findings of this study can be leveraged to promote a cultural shift towards a more positive perception of intraoperative coaching. 
S012

\section{RESIDENT PERFORMANCE IN SIMULATION IS CORRELATED WITH OPERATING ROOM PERFORMANCE FOR LAPAROSCOPIC CHOLECYSTECTOMY}

Yohei Kojima, $\mathrm{MD}, \mathrm{PhD}^{1}$; Harry J Wong, $\mathrm{MD}^{2}$; Kristine Kuchta, $\mathrm{MS}^{3}$; Stephen Haggerty, MD, FACS ${ }^{3}$; John Linn, MD, FACS ${ }^{3}$; Woody Denham, MD, FACS ${ }^{3}$; Michael Ujiki, MD, FACS $^{3} ;{ }^{1}$ Northshore University HealthSystem; Kyorin University Hospital, Japan; ${ }^{2}$ Northshore University HealthSystem; University of Chicago Medicine; ${ }^{3}$ Northshore University HealthSystem.

Background: Simulation is widely utilized in surgical training and has been shown to improve skills and confidence. However, little evidence exists to demonstrate transferability of skills obtained in the simulation setting to the operating room (OR). This study explores the association between resident simulation performance and performance in the OR in a laparoscopic cholecystectomy (LC) simulation module. Methods: A simulation module focused on LC utilizing a virtual reality simulator was completed by PGY2-3 general surgery residents at a single training program. Simulation performance was evaluated using the validated Global Operative Assessment of Laparoscopic Skills (GOALS) and Objective Structured Assessment of Technical Skills (OSATS), as well as a LC-specific simulation assessment form (LC-SIM). Residents' subsequent operating room performance of LC were measured by the Surgical Training and Assessment Tool (STAT), which is an online mobilebased evaluation (individual item score range 1-5) completed by attending surgeons immediately after case completion to evaluate residents' OR performance. Resident simulation data were compared with STAT data using multivariable mixed linear regression modeling, controlling for resident, attending, level of attending participation, complexity of case, and time from completion of simulation module.

Results: Twenty-one residents who completed the simulation module and also with STAT data on LC from 2016 to 2020 were included. Higher scores on incision/port placement on LC-SIM is associated with better tissue handling (coefficient $0.20, p=$ 0.048 ) and better time \& economy of motion on STAT (coefficient $0.22, \mathrm{p}=0.037$ ). However, higher scores on time and motion on OSATS is associated with worse tissue handling $(-0.28, p=0.046)$, worse time \& economy of motion $(-0.37, p=$ $0.009)$, and worse overall grade $(-0.21, \mathrm{p}=0.044)$. Higher scores on overall performance on OSATS is associated with worse time \& economy of motion $(-0.80, \mathrm{p}=$ $0.008)$. Higher scores on depth perception on GOALS is associated with worse tissue handling $(-0.28, \mathrm{p}=0.044)$

Conclusions: We found both significant direct and inverse relationships between resident simulation performance and OR performance, particularly in tissue handling and economy of motion. This suggests simulation performance does not reliably predict OR performance. However, this potentially highlights the concept of excessive caution in the real OR environment and longer operative time which could be interpreted as worse time and economy of motion by the attending surgeons.

\section{S013}

The impact of the current COVID-19 pandemic on the exposure of general surgery trainees to operative procedures

Saba Balvardi, MD; Mohsen Alhashemi, MD; Josie Cipolla, MS; Lawrence Lee, MD; Julio Fiore Jr., MD; Liane S Feldman, MD; McGill University.

Introduction: The objective of this study is to estimate the impact of current COVID-19 pandemic on the participation of general surgery trainees in operative procedures. The need to expand the healthcare capacity for patients with COVID-19 required a shutdown of elective surgery in our region in March 2020. To mitigate the negative effects of subsequent waves of the pandemic on surgical education, a thorough understanding of the magnitude and nature of its impact at each level of training is necessary.

Methods and Procedures: This study is a retrospective review of all emergency and elective general surgery procedures performed at the 3 sites of an academic health network between March and June in 2019 and 2020. Cases performed during the pandemic period (2020) were compared to the pre-pandemic period (2019). Procedures performed by a general surgeon involving adult patients were included. Trainees were categorized as junior (Post-Graduate-Year (PGY)1-2)or senior (PGY3-5). Operating exposure was defined as (1) the total number of trainees present in the operating room (OR) and (2) the total time spent in the OR by all trainees (hours). The impact of the pandemic was estimated as percentage of baseline (2019) and significance was tested using Exact Poisson Test.

Results: During the first wave of the COVID-19 pandemic, residents attendance in the OR decreased from 1328 to 914 cases $(68.9 \%$ of baseline; $p<0.001)$ and tota time in the OR decreased from 4000.4 to $2957.3 \mathrm{~h}(73.9 \%$ of baseline; $\mathrm{p}<0.001)$, with junior (622[2019] vs. 424 cases [2020]; $p<0.001)$ and senior $(803$ [2019] vs. 582 cases [2020]; $\mathrm{p}<0.001$ ) residents similarly affected. Exposure to Minimally Invasive Surgery and Bariatrics cases(490[2019] vs. 255[2020]; p < 0.001) was most negatively affected $(52.9 \%$ and $52.0 \%$ of baseline). However, the exposure to emergency surgery cases(211[2019] vs. 192[2020]; $p=0.1)$ and surgical oncology cases $(279[2019]$ vs. $251[2020] ; p=0.05)$ was relatively preserved $(91.0 \%$ and $90.0 \%$ of baseline).

Conclusions: The first wave of the COVID-19 pandemic reduced operative exposure by $30 \%$ for general surgery trainees. The pattern of this impact reflects institutional policies of prioritizing cancer operations and emergency surgeries. This knowledge can be used to design rotations or remediation activities to mitigate the impact of the pandemic on surgical training. 


\section{S014}

\section{SAGES Education Research: How are we doing?}

Tiffany N Anderson, MD, MHPE ${ }^{1}$; Robert Shi, MS, BA ${ }^{2}$; Aboubacar Kaba, BS ${ }^{3}$; Ladonna E Kearse, $\mathrm{MD}^{2}$; Elizabeth $\mathrm{M}$ Huffman, $\mathrm{MD}^{4}$; Ingrid S Schmiederer, $\mathrm{MD}^{2}$; E M Ritter, MD ${ }^{4}$; James R Korndorffer JR, MD, MHPE, FACS ${ }^{2}$; ${ }^{1}$ University of Florida; ${ }^{2}$ Stanford University; ${ }^{3}$ Saint Louis University; ${ }^{4}$ Indiana University.

Purpose: Deficiencies in the quality of surgical education scholarship are widely acknowledged. Education leaders have appealed for increased methodological rigor, including multi-institutional studies, attention to assessment validity evidence, and translation to clinically relevant outcomes. As the official journal of the Society of American Gastrointestinal and Endoscopic Surgeons and European Association for Endoscopic Surgery, Surgical Endoscopy (SE) publishes numerous manuscripts pertaining to surgical education. Thus, SE is an important resource for new evidence in education research. The purpose of this study is to assess the quality of education research published through SE and identify potential areas for improvement.

Methods: A PubMed search of SE examined all printed manuscripts categorized as education-related research from 2011 to 2019. The resulting titles and abstracts were reviewed to exclude purely qualitative studies, systematic reviews, meta-analyses, or publications without a research question. Using the established Medical Education Research Study Quality Instrument (MERSQI-such that scores can range from 5-18 points, with $>12$ generally associated with strong methodologic research), the remaining publications were then reviewed, classified, and scored by 7 raters (with 1 rater as a control). Intraclass correlation (ICC) was calculated for raters using MERSQI and data were examined using descriptive statistics.

Results: Of the 5328 studies identified, 233 met inclusion criteria. There was no significant difference in number of publications by year (average 25.88 [SD = 5.6]). Twenty-four percent (57) of studies were multi-institutional, The average MERSQI score was $12.5(\mathrm{SD}=2)$, range $6-17$, no difference between years. Forty-one percent (95) of studies scored 12 or less. Most studies used two group non-random design $(42.9 \%)$ or post/cross-sectional designs $(28.8 \%)$, whereas $36(15.5 \%)$ were randomized controlled trials. Analysis of validity evidence revealed that $93 \%$ (217) of manuscripts reported at least 1 measure of validity evidence (of variable reporting standards) and $28 \%$ (67) with 3 levels of validity evidence. Studies primarily reported changes in skills, knowledge, or behavior $(45 \%)$ or satisfaction or general facts $(44 \%)$, while patient-related outcomes encompassed $2.5 \%$ of studies. ICC was 0.93 (CI: $0.90-0.93, \mathrm{p}<0.001$ )

Conclusion: Review of manuscripts published recently shows the SE peer review process does facilitate the dissemination of education-related studies of moderate to good quality. This is a promising finding, despite deficits that were uncovered with potential for improvement, such as consistent presentation of validity evidence, levels of outcomes-based results, and randomized controlled trials. This journal's broad readership makes it an excellent venue for further advancement of educationrelated research.

\section{S015}

Implementation of a Virtual Basic Laparoscopic Surgery Course in Santa Cruz de la Sierra, Bolivia

Constance S Harrell Shreckengost, MD, $\mathrm{PhD}^{1}$; Erica Ludi, $\mathrm{MD}^{1}$; Alexandra Reitz, $\mathrm{MD}, \mathrm{MPH}^{1}$; Raul Rojas Aban, $\mathrm{MD}^{2}$; Lorena Juáregui Paravicini, $\mathrm{MD}^{2}$; Federico Serrot, MD ${ }^{1} ;{ }^{1}$ Emory University; ${ }^{2}$ Hospital de Niños "Dr Mario Ortiz Suárez", Santa Cruz de la Sierra, Bolivia.

INTRODUCTION: A surgical team from Bolivia and the United States implemented a virtual basic laparoscopic surgery course for Bolivian general and pediatric surgeons and residents during summer 2020. This simulation course aimed to enhance training in a lower-resource environment despite the challenges of decreased operative volume and lack of in-person instruction due to the coronavirus pandemic.

METHODS: A ten-week cognitive and technical laparoscopic surgery course was developed by collaboration between a Bolivian pediatric hospital and a US-based academic institution. Instruction occurred during seven live, video-based didactic sessions. Additional mentorship was provided through small group sessions. Pre- and post-course surveys and multiple-choice tests were distributed to evaluate participant confidence and knowledge and request course feedback. Changes in confidence and knowledge were analyzed by two-tailed t-tests. Finally, participants were encouraged to practice technical skills multiple times a week and submitted videos in the first week of the course and at course completion.

RESULTS: Of the thirteen enrolled participants, seven were practicing surgeons (5 men, 2 women), and six were pediatric surgery residents ( 2 men, 4 women). In the pre-course survey, the majority ( $n=8$ of 13) stated that they "almost never" practiced laparoscopic surgery. In the post-course survey, $100 \%$ respondents ( $n=8$ of 8 ) indicated that they found the course beneficial and anticipated performing more laparoscopic surgery in the future. Average scores on the cognitive test increased significantly $(49 \pm 12 \%$ to $62 \pm 10 \%, \mathrm{p}=0.016, \mathrm{n}=10$ for pre- and post-tests) Given the lack of standardized equipment needed for the intended quantitative evaluation, activity videos were assessed qualitatively, demonstrating moderate improvement. Reported confidence out of a 5-point scale significantly increased for three of the four activities: object transfer ( 1.8 vs $3.3, \mathrm{p}=0.001)$, intracorporeal knots ( 1.4 vs $2.8, \mathrm{p}=0.017)$ and precision cutting $(1.8$ vs $3.1, \mathrm{p}=0.022)$, with a trend towards increased confidence for extracorporeal knots $(1.7$ vs $2.8, \mathrm{p}=0.061)$.

CONCLUSION: Virtual international collaboration can improve knowledge and technical confidence for laparoscopic surgery, even in resource-limited settings during a global crisis. Future efforts should focus on standardizing resources for participants and enhancing access to live feedback and educational resources between classes. 


\section{S016}

\section{A Comparative Analysis of Video-based Surgical Assessment: Is Evaluation of the Entire Critical Portion of the Operation Necessary?}

Caleb W Barnhill, MD; Stephen J Kaplan, MD, MPH; Adnan A Alseidi, MD, EdM; Shanley B Deal, MD; Virginia Mason Medical Center.

Objective: The systematic, objective assessment of surgeon technical skill is imperative to ensuring high quality and safe surgical care. Video editing and file size, access to expert evaluators, and cost remain limitations in providing rapid and reliable operative assessments. Previous research has established the role, accuracy, and reliability of non-expert video-based operative evaluation. Our group sought to establish the equivalence of abbreviating video content for non-expert evaluation.

Methods: A video repository of six core general surgery procedures were submitted for evaluation by non-experts utilizing standard surgical performance assessment tools. Each procedure included three unique operative performances for a total of 18 videos in each protocol. The submissions were edited using four different abbreviating protocols based on the critical portion of the operation (Fig. 1). Protocol 0 included custom edited content which was deemed by the video editors to best represent the performance of the operating surgeon. Protocol 1 included the entire critical portion of the operation edited to $1-4 \times$ speed in order to condense the content evaluated to under $15 \mathrm{~min}$. Operative videos in protocol 2 were edited to include the first $10 \mathrm{~s}$ of every minute of the critical portion of the operation at $1 \times$ speed. Likewise, videos in protocol 3 included the first $20 \mathrm{~s}$ of every minute of the critical portion of the operation (at $1 \times$ speed). Aggregate performance assessment score for each video protocol was compared. A multivariable, multilevel mixedeffects model was constructed to predict total skill assessment scores.

Results: There was no difference in aggregate median score among the four study protocols: custom, 15.7 (14.4-16.2); condensed, 15.8 (15.2-16.4); 10-s, 15.8 (15.3-16.1); 20-s, 16.0 (15.1-16.3); $\mathrm{p}=0.65$. Regression modeling demonstrated a significant, but clinically-negligible effect of the 10 -s and 20 -s editing protocols versus the custom method on individual video score: condensed, +0.33 ($0.05-0.70), \mathrm{p}=0.09 ; 10-\mathrm{s},+0.29(0.04-0.55), \mathrm{p}=0.03 ; 20-\mathrm{s},+0.40(0.15-0.66), \mathrm{p}$ $=0.002$.

Conclusion: Standardized video editing protocols to abbreviate operative video length yields equivalent evaluations of surgical performance. Abbreviated video submissions with automated editing may decrease the cost and burden of videobased surgical evaluation while maintaining reliable assessment of operative skills.

Figure 1.

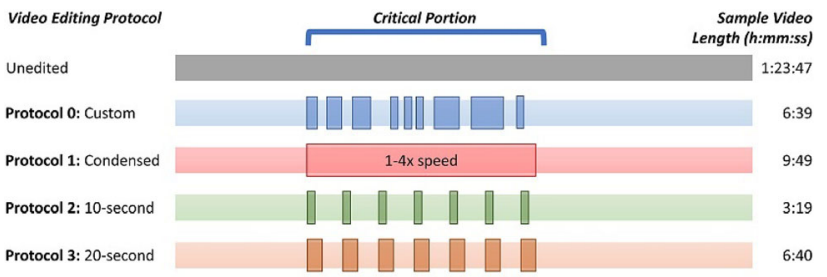

S017 The Entrustable Professional Activities of Laparoscopic Surgery
- moving towards an integrated training model

Carlos Gomez-Garibello, PhD; Maryam Wagner, PhD; Melissa Hanson, MD, FRCSC; Melina Vassiliou, MD, FRCSC; McGill University.

Introduction: Contemporary approaches to teaching and assessment in residency education propose the organization of competencies into meaningful units or Entrustable Professional Activities (EPAs). These units integrate multiple competencies within tasks that capture professional activities specific to a field. There are no EPAs that capture the full breadth of Laparoscopic Surgery (LS). The purpose of this research was to develop a framework that defines LS and integrates the essential competencies (knowledge, skills, and attitudes) that comprise it while remaining generic with regards to procedure and specialty.

Methods and Procedures: We designed a mixed-methods study to develop EPAs to capture the essential aspects of LS. Four education retreats were organized with subject matter experts (SMEs) in LS from various specialties working alongside surgical educators. The first retreat focused on generating a list of requisite fundamental, non-procedure-specific clinical skills related to laparoscopic surgery. This list was generated through a series of brainstorming activities, and cognitive task analyses. The second retreat employed a series of innovative think aloud activities to generate additional tasks associated with LS. Competencies were then developed by SMEs after analyses of all of these protocols, and then combined into EPAs. The fourth retreat focused on work with SMEs to identify the knowledge, skills and attitudes specific to basic LS for each of the EPAs. The last one focused on refining the EPAs and describing the observable behaviours related to them.

Results: Ten EPAs and associated observable behaviours and competencies specific to LS were identified. For instance, EPA 8 "Establish and maintain pneumoperitoneum" was associated with observable behaviours such as: i) manages pneumoperitoneum in response to changes in patient physiology, and ii) establishes pneumoperitoneum and adapts technique and settings to patient characteristics. In addition, specific knowledge areas were identified related to each EPA.

Conclusions: A comprehensive list of EPAs for LS was created. These EPAs can serve as a framework to guide the development of curriculum, clinical teaching, and assessment in any surgical program with a laparoscopic training component. They can be applied to any level of training by defining the expected observable behaviors and competencies associated with a given level of expertise. 


\section{S018}

Racial Disparities Analysis in Bariatric Surgery: Self-Identified Black Patients Have No Difference in Access to Minimally Invasive Fundoplication But Face Longer Hospital Stay After Fundoplication

Syed F Haider, $\mathrm{MD}^{1}$; Kasey L Wood, $\mathrm{BS}^{2}$; Mario M Matabele, $\mathrm{BS}^{2}$; Sirui Ma, MD ${ }^{1}$; Archana Babu, BA, MS ${ }^{1}$; Aziz Merchant, MD' ${ }^{1}$; Ravi J Chokshi, MD, MPH, FACS $^{3} ;{ }^{1}$ Department of Surgery, Rutgers New Jersey Medical School; ${ }^{2}$ University of Wisconsin School of Medicine and Public Health; ${ }^{3}$ Department of Surgical Oncology, Rutgers New Jersey Medical School.

Background: Fundoplication is used to treat refractory gastroesophageal reflux disease (GERD). Minimally invasive fundoplication is both safe and effective in treating patients with severe GERD and is now considered standard of care procedure. Literature shows that minimally invasive procedure is associated with shorter length of stay and fewer early and late complications. This study aims to identify racial disparities in the different approaches to fundoplication. We further evaluate the relationship between the race and postoperative complications.

Methods: The ACS-NSQIP database was queried to isolate all cases of fundoplication between 2012 and 2017. Cases with primary CPT code 43,280 were scored as laparoscopic approach, and cases with CPT code 43,325, 43,327, and 43,328 were scored as open approach. Patient demographic, preoperative, operative, and 30-day postoperative data were obtained. 30-day postoperative complications were grouped as follows: any complication, minor complication, severe complication, unplanned readmission, unplanned reoperation, and death. A propensity score matching algorithm was then implemented to generate a clinically balanced cohort of patients in which every patient of black self-identified race was matched to five patients of white self-identified race. Chi-squared tests were then used on this balanced cohort in order to identify any existing racial disparities in access to laparoscopic approach to surgery, likelihood of postoperative complications, and length of hospital stay. Results: There were 9,530 patients of white self-identified race and 569 patients of black self-identified race who met inclusion criteria. Propensity score matching generated a cohort of 2,845 patients of white self-identified race and 569 patients of black self identified race. Analyses of this matched cohort revealed no significant racial disparity in access to laparoscopic fundoplication or in the incidence of postoperative complications. However, black patients faced a significantly longer hospital stay $(2.02 \pm 3.39$ days for white patients versus $2.96 \pm 5.44$ days for black patients, $\mathrm{p}<0.001$

Conclusions: No significant racial disparity in access to Minimally Invasive Fundoplication was identified. However, black race had statistically significant longer hospitalization stay resulting in increased cost.

\section{S019}

\section{Patient-Reported Bowel and Bladder Function Are Not Adversely Impacted by Bariatric Surgery}

Michelle Campbell, $\mathrm{MD}^{1}$; Eliza Conaty, $\mathrm{MA}^{2}$; Hoover Wu, $\mathrm{MD}^{1}$; Harry J Wong, $\overline{M D}^{1}$; Mikhail Attaar, MD ${ }^{1}$; Kristine Kuchta, $\mathrm{MS}^{1}$; Stephen P Haggerty, $\mathrm{MD}^{1}$, Woody Denham, $\mathrm{MD}^{1}$; John G Linn, $\mathrm{MD}^{1}$; Zeeshan Butt, $\mathrm{PhD}^{3}$; Michael Ujiki, $\mathrm{MD}^{1} ;{ }^{1}$ NorthShore University Health System; ${ }^{2}$ Loyola University Chicago Stritch School of Medicine; ${ }^{3}$ Northwestern University Feinberg School of Medicine.

Introduction: This study aims to assess the effect of bariatric surgery on patientreported outcomes of bowel and bladder function. We hypothesized that bariatric surgery does not worsen bowel and bladder function.

Methods and Procedures: A retrospective review was conducted of a prospectively maintained surgical quality database. We included patients who underwent primary bariatric surgery at a single institution between 2012 and 2020, excluding revisional procedures. Patient-reported outcomes were assessed using Surgical Outcomes Measurement System (SOMS) bowel and bladder function questionnaires at time of pre-operative consult, and routine post-operative follow-up visits at 2 weeks, 6 weeks, 3 months, 6 months, 1 year, and 2 years. Data were analyzed using a statistical mixed effects model.

Results: A total of 515 patients $(81.2 \%$ female) were identified with completed SOMS questionnaire data on bowel and bladder function. Of these, $344(66.8 \%)$ underwent gastric bypass, $165(32.0 \%)$ underwent sleeve gastrectomy, and $6(1.2 \%)$ underwent either gastric banding or duodenal switch. Compared to pre-operative baseline scores, patients report a transient worsening of bowel function at 2-weeks post-op ( $p=0.004)$. However, by 3-month follow-up visit, bowel function improves and is significantly better than baseline $(p=0.006)$; this improvement is sustained at every point through 2 -year follow-up $(p=0.014)$. Bladder function scores improve immediately at 2-weeks post op $(p=0.008)$ and also show sustained improvement at subsequent follow-up. On subgroup analysis, sleeve patients show greater improvement than bypass patients at 1 -year on bowel function scores $(p=0.035)$. Multivariable analysis shows an improvement in bowel function associated with greater percent excess weight loss $(\% \mathrm{EWL})(p=0.059)$.

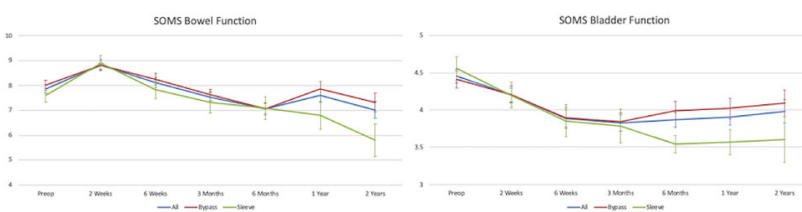

Figure 1 SOMS scores for bowel function (minimum 4) and bladder function (minimum 3) over time. Note: lower scores indicate better function

Conclusions: Bariatric surgery does not worsen patient-reported bowel or bladder function. In fact, there is an overall improvement from baseline pre-operative scores for both bowel and bladder function by 3-months post-op which is sustained at 2-year follow-up. The transient worsening of bowel function at 2-weeks post-op is likely due to narcotic effects after surgery. Most encouragingly, a greater \%EWL shows a trend toward significantly improved bowel function after bariatric surgery. 
S020

Comparative Effectiveness of Sleeve Gastrectomy vs Roux-En-Y Gastric Bypass in Patients Giving Birth After Bariatric Surgery: Reinterventions and Maternal Outcomes

Grace F Chao, MD, $\mathrm{MSc}^{1}$; Jie Yang, $\mathrm{PhD}^{2}$; Alex Peahl, MD, MSc ${ }^{3}$; Jyothi Thumma, $\mathrm{MPH}^{2}$; Justin B Dimick, MD, $\mathrm{MPH}^{4}$; David E Arterburn, MD, $\mathrm{MPH}^{5}$; Dana A Telem, MD, MPH ${ }^{4} ;{ }^{1}$ Veterans Affairs Ann Arbor; ${ }^{2}$ Center for Healthcare Outcomes and Policy; ${ }^{3}$ University of Michigan Department of Obstetrics and Gynecology; ${ }^{4}$ University of Michigan Department of Surgery; ${ }^{5}$ Kaiser Permanente Washington.

Introduction: Women of childbearing age comprise approximately $65 \%$ of all patients who undergo bariatric surgery in the United States every year, yet data on maternal outcomes for patients who conceive within two years of surgery are limited. Additionally, it is unknown whether these outcomes differ by procedure. To better counsel women who become pregnant, elucidating the surgical and pregnancy risks for those women who give birth after bariatric surgery is needed.

Methods: Using IBM MarketScan claims data, we performed a retrospective cohort study of all women age 18-52 undergoing laparoscopic sleeve gastrectomy or laparoscopic Roux-en-Y gastric bypass from 2011-2017 with 2-year continuous follow-up after surgery. We utilized multivariable logistic regression, controlling for age, U.S. state, and Elixhauser comorbidities, to examine the association between giving birth and risk of reinterventions (revisions, enteral access, vascular access, reoperations, other). We then examined the association between procedure type and maternal pregnancy outcomes using multivariable logistic regression, controlling for age, U.S. state, and the Bateman comorbidity index. Pregnancy outcomes included were hyperemesis gravidarum, gestational diabetes, pre-eclampsia, eclampsia, iron deficiency anemia, pre-term labor, prolonged labor, post-partum hemorrhage, peripartum hysterectomy, higher order perineal laceration, major puerperal infections, and anesthesia complications.

Results: There were 38,922 women who underwent bariatric surgery. Of these, 1,079 women gave birth. Reoperation rates were $12.1 \%$ in women who did not give birth compared to $13.5 \%$ in women who gave birth. Among women who gave birth, $56 \%$ of reinterventions occurred prior to pregnancy and $13 \%$ occurred during pregnancy. Almost all reinterventions that occurred during pregnancy were biliary operations. Multivariable logistic regression analysis showed no association between post-bariatric birth and reintervention rate (OR: $0.93,95 \% \mathrm{CI}$ : 0.78-1.12, $\mathrm{P}=0.45)$. There was no significant difference based on procedure type.

We then examined pregnancy outcomes in the patients who gave birth after bariatric surgery. Laparoscopic sleeve gastrectomy was associated with lower rates of gestational diabetes than Roux-en-Y gastric bypass (OR: 3.51, 95\%CI: 1.77-6.93, P < $0.001)$. There were no statistically significant differences between the two procedures in the other pregnancy outcomes.

Conclusion: In this first nationally representative cohort study of surgical outcomes in patients who give birth after bariatric surgery in the United States, we found that giving birth was not associated with an increased risk of reinterventions. Additionally, the pregnancy outcomes were comparable to those for women with BMI of $35 \mathrm{~kg} / \mathrm{m}^{2}$ or greater. Thus, patients may be able to safely complete pregnancy within the first 2 years after surgery.
S021

\section{POSTOPERATIVE BLEEDING IN SLEEVE GASTRECTOMY IS INDEPENDENT OF STAPLE HEIGHT AND STAPLE LINE SUTURING}

Aman Ali, MD; Jeffrey Hodges, MD; Farshad Amirkhosravi, MD; Rukan Huq; Sara Yasrebi; John Paek, DO; Patrick Reardon, MD, FACS; Houston Methodist Hospital.

Background: Sleeve gastrectomy is recognized as an effective treatment modality for morbid obesity. Over 100,000 sleeve gastrectomy procedures are performed annually in the United States, with some patients suffering from postoperative bleeding complications. This study assessed the influence of patient-specific and perioperative factors on postoperative bleeding complications.

Methods: This is a single-center retrospective cohort study of 1213 sleeve gastrectomy procedures performed between 2005 and 2019. There were 52 high-risk patients excluded due to end stage renal disease, cirrhosis, and anticoagulation use for left ventricular assist device. Patient demographics, comorbidities, and procedure details including type of energy device, staple type, staple height, staple line suturing (none, complete, selective), and staple line clipping were compared for postoperative blood transfusion and bleeding using multiple logistic regression. Postoperative bleeding was defined by requiring blood transfusion and takeback to the operating room for hemostasis

Results: There were 1161 patients who underwent sleeve gastrectomy and passed the exclusion criteria. Of these 1161 patients, twenty-five (2.2\%) received postoperative blood transfusion and nine $(0.8 \%)$ had postoperative bleeding requiring takeback to the operating room. The median age was statistically higher for patients with postoperative blood transfusion ( 43 vs $50 ; \mathrm{p}=0.01$ ) and postoperative bleeding (43 vs $58 ; p=0.03$ ). There was no statistical difference in postoperative blood transfusion or bleeding with different energy devices $(p=0.82)$, staple types $(p=$ $0.16)$, staple heights $(0.38)$, and staple line suturing or clipping $(\mathrm{p}=0.92)$ (Table 1$)$. In addition, there was no difference in bleeding when comparing no over-sewing, selective over-sewing, and complete over-sewing of the staple line $(\mathrm{p}=0.44)$. In addition, patients with postoperative bleeding had longer hospital length of stay by 2 additional days $(\mathrm{p}<0.001)$.

Conclusion: Sleeve gastrectomy procedure has tremendous variability in surgical technique. We observed no decrease in postoperative blood transfusion or bleeding with over-sewing the staple line or with different staple heights and staple adjuncts. Patient selection is crucial, as patient's age and coagulopathic comorbidities are observed to lead to higher postoperative bleeding complications.

Table 1: Postoperative bleeding and blood transfusion

\begin{tabular}{|c|c|c|c|c|c|c|c|}
\hline & $\begin{array}{c}\text { Total } \\
\text { (N=1161) }\end{array}$ & \begin{tabular}{|c|} 
No bleeding \\
(n=1152)
\end{tabular} & $\begin{array}{l}\text { Bleeding } \\
(n=9)\end{array}$ & p-value & $\begin{array}{l}\text { No PRBC } \\
(n=1136)\end{array}$ & $\begin{array}{l}\text { PRBC } \\
(n=25)\end{array}$ & p-value \\
\hline Energy Device & & & & 0.49 & & & 0.82 \\
\hline Ligasure & $306(26.4)$ & $303(26.3)$ & $3(33.3)$ & & $298(26.2)$ & $8(32.0)$ & \\
\hline Enseal & $223(19.2)$ & $220(19.1)$ & $3(33.3)$ & & $220(19.4)$ & $3(12.0)$ & \\
\hline Harmonic & $604(52.0)$ & $601(52.2)$ & $3(33.3)$ & & $590(51.9)$ & $14(56.0)$ & \\
\hline Vessel sealer & $15(1.3)$ & $15(1.3)$ & $0(0.0)$ & & $15(1.3)$ & $0(0.0)$ & \\
\hline Unknown & $13(1.1)$ & $13(1.1)$ & $0(0.0)$ & & $13(1.1)$ & $0(0.0)$ & \\
\hline Staple type & & & & 0.56 & & & 0.16 \\
\hline Ethicon & $800(68.9)$ & $795(69.0)$ & $5(55.6)$ & & $785(69.1)$ & $15(60.0)$ & \\
\hline Covidien & $336(28.9)$ & $332(28.8)$ & $4(44.4)$ & & $327(28.8)$ & $9(36.0)$ & \\
\hline Sureform & $11(0.9)$ & $11(1.0)$ & $0(0.0)$ & & $11(1.0)$ & $0(0.0)$ & \\
\hline Seamguard & $4(0.3)$ & $4(0.3)$ & $0(0.0)$ & & $3(0.3)$ & $1(4.0)$ & \\
\hline Unknown & $10(0.9)$ & $10(0.9)$ & $0(0.0)$ & & $10(0.9)$ & $0(0.0)$ & \\
\hline First Staple Height & & & & 0.77 & & & 0.38 \\
\hline Blue Ethicon & $32(2.8)$ & $32(2.8)$ & $0(0.0)$ & & $32(2.9)$ & $0(0.0)$ & \\
\hline Gold Ethicon & $249(21.8)$ & $247(21.8)$ & $2(22.2)$ & & $245(22.0)$ & $4(16.0)$ & \\
\hline Green Ethicon & $482(42.2)$ & $479(42.3)$ & $3(33.3)$ & & $472(42.3)$ & $10(40.0)$ & \\
\hline Black Ethicon & $35(3.1)$ & $35(3.1)$ & $0(0.0)$ & & $34(3.0)$ & $1(4.0)$ & \\
\hline Gold Covidien & $3(0.3)$ & $3(0.3)$ & $0(0.0)$ & & $3(0.3)$ & $0(0.0)$ & \\
\hline Purple Covidien & $113(9.9)$ & $111(9.8)$ & $2(22.2)$ & & $110(9.9)$ & $3(12.0)$ & \\
\hline Black Covidien & $217(19.0)$ & $215(19.0)$ & $2(22.2)$ & & $211(18.9)$ & $6(24.0)$ & \\
\hline Green Sureform (robotic) & $5(0.4)$ & $5(0.4)$ & $0(0.0)$ & & $5(0.4)$ & $0(0.0)$ & \\
\hline Blue Gore Seamguard & $1(0.1)$ & $1(0.1)$ & $0(0.0)$ & & $1(0.1)$ & $0(0.0)$ & \\
\hline Black Seamguard & $4(0.4)$ & $4(0.4)$ & $0(0.0)$ & & $3(0.3)$ & $1(4.0)$ & \\
\hline Number of staples & $6.0(5.0,6.0)$ & $6.0(5.0,6.0)$ & $5.0(5.0,6.0)$ & 0.21 & $6.0(5.0,6.0)$ & $6.0(5.0,6.0)$ & 0.85 \\
\hline Suture & & & & 0.44 & & & 0.92 \\
\hline Nothing & $268(23.1)$ & $265(23.0)$ & $3(33.3)$ & & $262(23.1)$ & $6(24.0)$ & \\
\hline Selective suturing & $96(8.3)$ & $95(8.2)$ & 1 (11.1) & & $93(8.2)$ & $3(12.0)$ & \\
\hline Entire suturing & $711(61.2)$ & $707(61.4)$ & $4(44.4)$ & & $696(61.3)$ & $15(60.0)$ & \\
\hline Clip placement & $65(5.6)$ & $64(5.6)$ & $1(11.1)$ & & $64(5.6)$ & $1(4.0)$ & \\
\hline Selective suturingtelip placement & $1(0.1)$ & $1(0.1)$ & $0(0.0)$ & & $1(0.1)$ & $0(0.0)$ & \\
\hline Entire suturingtelip placement & $13(1.1)$ & $13(1.1)$ & $0(0.0)$ & & $13(1.1)$ & $0(0.0)$ & \\
\hline Unknown & $7(0.6)$ & $7(0.6)$ & $0(0.0)$ & & $7(0.6)$ & $0(0.0)$ & \\
\hline
\end{tabular}


S022

\section{Long-term Abdominal Complaints Following Bariatric Surgery}

Tarin C Worrest, MD; Nancy Puzziferri, MD; Elizabeth N Dewey, MS; Farah Husain, MD; Oregon Health and Science University.

INTRODUCTION: Bariatric surgery has been anecdotally associated with increased gastrointestinal complaints. However, there have been few prospective studies systematically looking at these symptoms. The Longitudinal Assessment of Bariatric Surgery 2 (LABS-2) study reports long-term bariatric outcomes including quality of life and gastrointestinal symptoms. The aim of this study was to determine if bariatric surgery changed a range of gastrointestinal symptoms, including nausea, reflux, bloating, and bowel changes, as measured by responses on the Gastrointestinal Symptom Rating Scale (GSRS) over time.

METHOD: Methods for the LABS-2 study were previously described. Participants without a baseline or follow up GSRS, or who underwent a procedure other than a primary bariatric surgery were excluded. Participants completed surveys prior to bariatric surgery and at six yearly follow-up visits. The GSRS evaluates 15 abdominal symptoms on a 7-pt Likert scale. GSRS survey responses were summed, and the total score analyzed. Scores were aggregated into symptomatic domains. Follow-up visit GSRS was compared to baseline, and differences were evaluated with t-tests. Significance was assessed at $\mathrm{p}<0.05$. Analysis was conducted in JMP 15.2 (SAS Inc., Cary, NC).

RESULTS: 2,467 participants enrolled in LABS-2, and 2,053 were included in the study; 1,496 Roux-en-Y gastric bypass (RYGB), 512 laparoscopic adjustable gastric band (LAGB), and 45 sleeve gastrectomy (SG). The baseline mean total GSRS score for all participants was 28.3 (95\% CI: 27.8-28.8). Mean total GSRS score decreased significantly at 12 months (mean: $27.1,95 \%$ CI: 26.6-27.6, p <0.0001), but was not significantly different from baseline at all follow-up visits (Fig. 1). Results were similar for RYGB and LAGB. For RYGB, baseline total score was 28.7 (28.1-29.3), and decreased to $27.3(26.7-27.9)$ at 1 year $(\mathrm{p}=0.0002)$. For LAGB, baseline score was 27.3 (26.4-28.2), and decreased to $26.3(25.4-27.2)$ at 1 year $(\mathrm{p}=0.04)$. Similarly, there were no differences from baseline at all follow-up visits. For SG, mean total GSRS was not significantly different from baseline at all follow-up visits. Within the GSRS domains, there was no difference from baseline score at any follow-up time point for any procedure.

CONCLUSIONS: This large, longitudinal cohort study suggests that gastrointestinal symptoms, overall, do not worsen after bariatric surgery. Rather, participants can expect a slight improvement in gastrointestinal symptoms around one year following RYGB or LAGB.

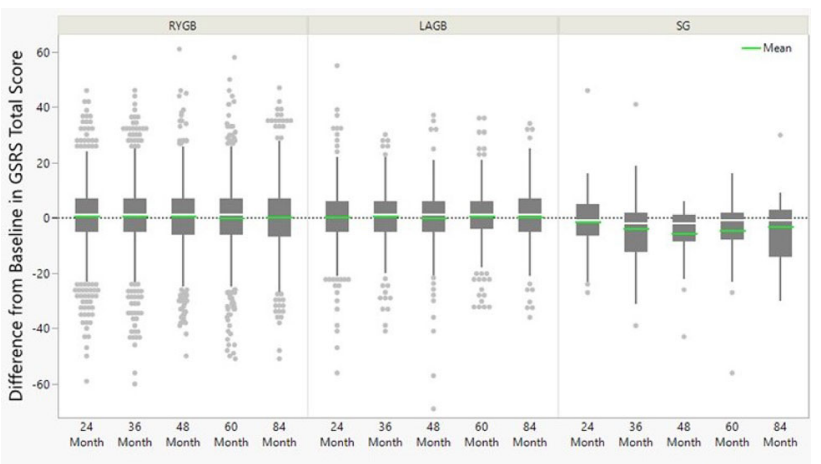

Fig. 1 Gastrointestinal Symptom Rating Scale (GSRS) scores for Roux-en-Y gastric bypass (RYGB), laparoscopic adjustable gastric band (LAGB), and sleeve gastrectomy (SG) patients. There was no statistically significant change at any time point

\section{S023}

Telemedicine Utilization in Response to COVID-19 Among Surgical Clinics at an Academic Tertiary Care Center

Jay Zhu, MD; Baraka M Gitonga; Roccio E Carrera, MD; Rebecca P Petersen, MD; Judy Y Chen-Meekin, MD; Saurabh Khandelwal, MD; Brant K Oelschlager, MD; Andrew S Wright; University of Washington Medical Center.

Introduction: Widespread adoption of telemedicine for surgical outpatient visits is a new phenomenon driven by the COVID-19 pandemic. We aimed to describe telemedicine utilization patterns in general surgery clinics at an academic tertiary care center serving a multi-state region.

Methods: A retrospective chart review was performed on all clinic visits conducted from January to September 2020 in four representative surgical subspecialty clinics including: 1) Acute care surgery 2) Minimally invasive surgery, 3) Bariatric surgery, and 4) Community general surgery. Detailed demographic data was collected for all patient visits conducted by these clinics in July and August 2020. A chi-squared test of independence was performed to examine the relationship between demographic variables and participation in telemedicine visits.

Results: We identified 6692 patient visits across the four examined surgical clinics. Total outpatient visits per month reached a nadir in April 2020 (65\% decrease in volume when compared to January 2020) with a sharp increase in telemedicine utilization during the same month (37.8\% of all visits compared to $0.8 \%$ of all visits in the month prior). Higher rates of telemedicine adoption were observed in our subspecialty MIS and bariatric surgery clinics $(60.4 \%$ and $51.9 \%$, April-September) compared to our ACS and community general surgery clinics $(10.4 \%$ and $11.8 \%)$. Examining the 1548 patient visits conducted in July and August of 2020, women were 1.62 times more likely than men to participate in telemedicine $(\mathrm{p}=0.0002)$ Patients insured by private insurance were 1.51 times more likely to participate in telemedicine than those insured by Medicaid $(\mathrm{p}=0.04)$. There was no difference in telemedicine participation between patients insured by private insurance and those insured by Medicare $(\mathrm{p}=0.7)$. White patients were 1.54 times more likely to participate in telemedicine compared to non-white patients $(\mathrm{p}=0.01)$.

Conclusion: We observed a wide variation in the rates of telemedicine utilization among different clinics at our academic tertiary care center. We also found that in the middle of the COVID-19 pandemic, certain groups had significantly lower rates of telemedicine participation, including male patients, non-white patients, and patients insured by Medicaid. This may be due to differing attitudes toward or access to technology among certain populations but this also raises concern for potential bias in the way in which telehealth services are implemented and offered to patients. These results provide a basis for future studies centered on reducing barriers and defining best practices associated with telemedicine utilization in surgical clinics.

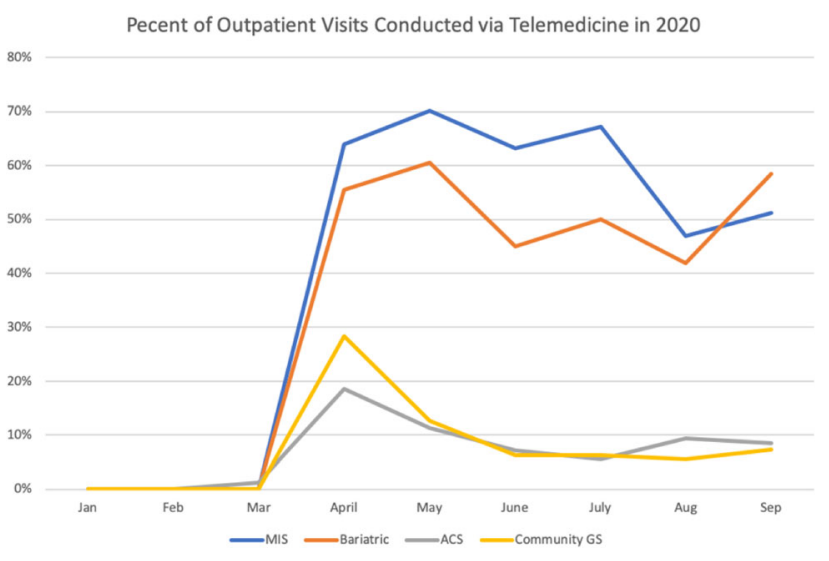




\section{S024}

\section{Mitigating the Impact of COVID-19: Efforts by the Fellowship Council to Ensure Adequate Training Experience}

Madhuri B Nagaraj, MD, MS ${ }^{1}$; Daniel J Scott, MD ${ }^{1}$; Juliet G Holder-Haynes, MD ${ }^{2}$; Adnan Alseidi, MD, EdM ${ }^{5}$; L Michael Brunt, $\mathrm{MD}^{4}$; D Rohan Jeyarajah, MD ${ }^{5}$; Shanu N Kothari, MD, FACS ${ }^{6}$; Aurora D Pryor, MD, MBA, FACS ${ }^{7}$; Michael A Schweitzer, $\mathrm{MD}^{8}$; Samer G Mattar, MD, FACS ${ }^{2} ;{ }^{1} \mathrm{UT}$ Southwestern; ${ }^{2}$ Baylor College of Medicine; ${ }^{3}$ University of California at San Francisco; ${ }^{4}$ Washington University School of Medicine; ${ }^{5} \mathrm{TCU}$ and UNTHSC School of Medicine; ${ }^{6}$ USC School of Medicine; ${ }^{7}$ Stony Brook Medicine; ${ }^{8}$ Johns Hopkins Center for Bariatric Surgery.

Background: In March 2020, the cancellation of elective operations due to COVID19 led to the concern that some fellows may not meet minimum training requirements. The Fellowship Council (FC) was uniquely positioned through its support from multiple societies and expansive case log database to closely monitor training patterns and intervene if needed. Our aims were to analyze case logs trends to delineate the impact of COVID-19 as a guide to enacting mitigation measures.

Methods: The FC conducted monthly deliberations with the sponsoring societies of the FC to monitor and manage the situation, including modifying training requirements if needed. De-identified case log records were obtained from all one-year programs for 2016-2020. Programs designations or types with fewer than ten fellows in any given year were excluded from analysis. Retrospective analysis of case log trends was performed, with an emphasis on total cases per fellow, further determined by month and program type. Comparisons were performed between 2016-2019 and 2019-2020 academic years. Non-parametric statistical analysis was performed using the Mann-Whitney-U test in R-Studio ${ }^{\circledR}$.

Results: A total of 5 program types, 151 programs and 705 fellows were analyzed. Decline in case numbers due to COVID-19 were noted starting February 2020 through April and returned to baseline by June 2020 (Figure). When comparing March-July 2018-2019 and August-December 2019-2020, average monthly cases per fellow were 20.85 and 23.32 respectively (p-value $=0.061$ ). However, when comparing the academic year 2019-2020 and 2016-2019 in aggregate, there was a significant reduction in cases per fellow per year in Advanced GI ( $\mathrm{p}$-value $=0.028$ ), Advanced GI MIS ( $\mathrm{p}$-value $=0.007$ ), and Advanced GI MIS/Bariatrics ( $\mathrm{p}$-value = 0.001 ) with a decrease of $13-19 \%$ in total case volume. No reduction was seen in Colorectal/MIS ( $\mathrm{p}$-value $=0.534$ ) and Bariatrics fellowship case volume ( $\mathrm{p}$-value $=$ $0.827)$, which had a $0-6 \%$ increase.

Conclusions: The outbreak of COVID-19 had a dramatic impact on the training experience of a segment of this cohort. Fellow experience was preserved largely due to mitigating measures adopted by the sponsoring societies of the FC and the eventual recovery of elective case numbers. Three program types specifically experienced significant decrements in case volumes, but FC and sponsoring society allowances successfully lessened these effects. As COVID-19 continues to pose a threat to fellowship training, these data underscore the importance of the FC in safeguarding the fellowship experience.

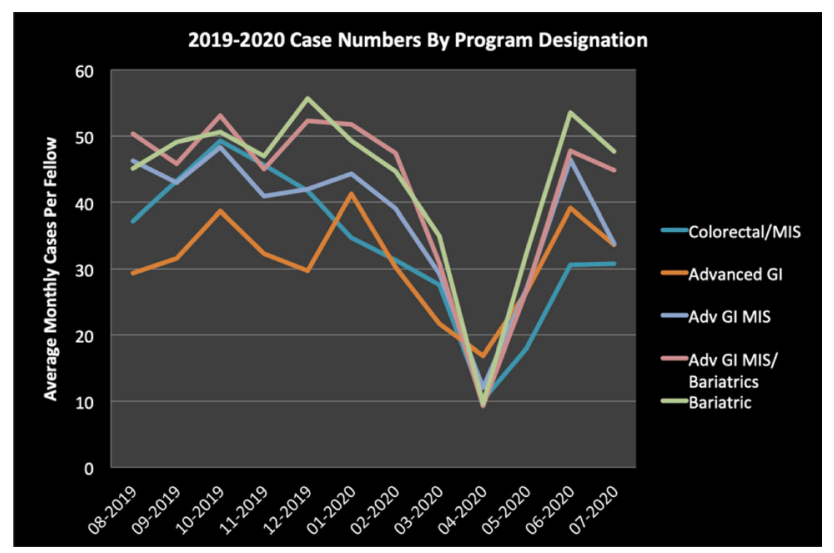

S025

\section{Clinician Burnout and Post Traumatic Stress Disorder during the COVID-19 Pandemic}

Maysoon Gamaleldin, MD; Judith Scheman, PhD; Sofya Asfaw, MD; Kelly Nimylowycz, MHA; Silvia Perez-Protto, MD; Conor P Delaney, MD, PhD; Ajita Prabhu, MD; Cleveland Clinic Foundation.

Introduction: The coronavirus pandemic (COVID 19) originated in Wuhan, China and is rapidly evolving across the world. Coupled with an epidemic of healthcare worker burnout, there is an urgent need to determine the scope of burnout and PTSD in the healthcare workforce. We aimed to identify the incidence of PTSD and burnout in healthcare workers at a quarternary referral center as a first step towards building a program around support for these issues.

Methods: We conducted a cross-sectional anonymous online survey within the digestive disease insitute at a quaternary referral center in July 2020, approximately 4 months after a state wide lockdown. The survey was sent to attending physicians, critical care fellows and advanced practice providers. We included two questionnaires in the survey: the Post Traumatic Stress Disorder (PTSD) Checklist PCL-5 and the Stanford Professional Fulfillment and Burnout (PFI) questionnaire. A score of $\geq 14$ for the burnout items on the PFI questions indicates burnout; where a score of $\geq 33$ on the PCL- 5 checklist indicates probable PTSD. Additional demographics were collected including age groups, gender, job title, workload and years of clinical experience.

Results: Of the 316 surveys sent, a total of $131(41.5 \%)$ responses were received: 35 $(26.7 \%)$ caregivers from critical care, $12(9.16 \%)$ from colorectal surgery, $32(24.4 \%)$ from Gastroenterology, and 52(39.7\%) from General Surgery. The overall incidence of burnout amongst all departments was $28.2 \%$; with females being more likely to develop burnout $(78.4 \%, \mathrm{p}=0.001)$. Burnout was also more prevalent in the $30-39$ age group (42\%) and the 40-49 group (38.4\%). Positive screen for PTSD incidence was $9.2 \%$ with women also being more likely to report symptoms $(83.3 \%, \mathrm{p}=0.003)$ (Table 1). $52.7 \%$ of the caregivers reported professional fulfillment. Concerns for child care, contracting COVID 19, and increased workload were the most reported concerns.

Conclusion: The COVID 19 pandemic has had a significant impact on healthcare workers' mental health. Institutions should continue to support their staff with regular check-ins and continuous efforts to improve engagement and communication. Interventions targeted to address caregivers concerns should be considered to help reduce stress. 


\begin{tabular}{|c|c|c|c|c|c|c|c|}
\hline \multirow[t]{2}{*}{$n(K)$} & [ALL] & $\begin{array}{l}\text { No } \\
\text { Burnout }\end{array}$ & Burnout & \multirow{2}{*}{$\begin{array}{c}\text { Overall p } \\
\text { value }\end{array}$} & $\begin{array}{l}\text { No } \\
\text { PTSD }\end{array}$ & $\begin{array}{l}\text { Probable } \\
\text { PTSO }\end{array}$ & \multirow{2}{*}{$\begin{array}{c}\text { Overall p } \\
\text { value }\end{array}$} \\
\hline & $\mathrm{N}=131$ & $\mathrm{~N}=94$ & $\mathrm{~N}=37$ & & $\mathrm{~N}=119$ & $\mathrm{~N}=12$ & \\
\hline Age Group & & & & 0.449 & & & 0.343 \\
\hline $20-49$ years & $\begin{array}{l}91 \\
(69.5 \%)\end{array}$ & $\begin{array}{l}63 \\
{[67.0 \%]}\end{array}$ & $\begin{array}{l}28 \\
\{75.7 \%]\end{array}$ & & $\begin{array}{l}81 \\
{[68,1 \%]}\end{array}$ & $10(83.3 \%)$ & \\
\hline$\geq 50$ years & $\begin{array}{l}40 \\
{[30.5 \%)}\end{array}$ & $\begin{array}{l}31 \\
\mid 33,0 \%)\end{array}$ & $\begin{array}{l}9 \\
{[24,3 \%]}\end{array}$ & & $\begin{array}{l}38 \\
{[31.9 \%]}\end{array}$ & $2(16.7 \%)$ & \\
\hline Gender & & & & 0.001 & & & 0,003 \\
\hline Female & $\begin{array}{l}72 \\
{[55.0 \%]}\end{array}$ & $\begin{array}{l}43 \\
(45,7 \%)\end{array}$ & $\begin{array}{l}29 \\
(78,4 \%)\end{array}$ & & $\begin{array}{l}62 \\
(52,1 \%)\end{array}$ & $10(83.3 \%)$ & \\
\hline Male & $\begin{array}{l}58 \\
(44,3 \%)\end{array}$ & $\begin{array}{l}50 \\
(53.2 \%)\end{array}$ & 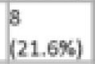 & & $\begin{array}{l}57 \\
(47.9 \%)\end{array}$ & $1(8.33 \%)$ & \\
\hline Non-binary & $\begin{array}{l}1 \\
\{0.76 \%)\end{array}$ & $1(1,06 \%)$ & $1_{\{0.00 \%\}}^{0}$ & & $\begin{array}{l}0 \\
10.00 \%\end{array}$ & $1(8.33 \%)$ & \\
\hline $\begin{array}{l}\text { Years in Clinica } \\
\text { Practice }\end{array}$ & & & & 0.922 & & & 0.231 \\
\hline $0-10$ years & $\begin{array}{l}54 \\
{[41.2 \%)}\end{array}$ & $\begin{array}{l}38 \\
\{40.4 \%)\end{array}$ & $\begin{array}{l}16 \\
(43.2 \%)\end{array}$ & & $\begin{array}{l}47 \\
\mid 39.5 \%]\end{array}$ & $7(58.3 \%)$ & \\
\hline$>10$ years: & $\begin{array}{l}77 \\
{[58.8 \%)}\end{array}$ & $\begin{array}{l}56 \\
{[59.6 \%\}}\end{array}$ & $\begin{array}{l}21 \\
\text { (56.8\%) }\end{array}$ & & $\begin{array}{l}72 \\
{[60,5 \%)}\end{array}$ & $5(41.7 \%)$ & \\
\hline $\begin{array}{l}\text { Children in the } \\
\text { Household }\end{array}$ & & & & 0.094 & & & 0.027 \\
\hline No & $\begin{array}{l}54 \\
(41.2 \%)\end{array}$ & $\begin{array}{l}34 \\
(36.2 \%)\end{array}$ & $\begin{array}{l}20 \\
(54,1 \%)\end{array}$ & & $\begin{array}{l}45 \\
{[37.8 \%)}\end{array}$ & $9(75.0 \%)$ & \\
\hline Yes & $\begin{array}{l}77 \\
\{58.8 \%)\end{array}$ & $\begin{array}{l}60 \\
\{63.8 \%\}\end{array}$ & $\begin{array}{l}17 \\
\{45.9 \%\end{array}$ & & $\begin{array}{l}74 \\
\{62.2 \%\}\end{array}$ & $3(25.0 \%)$ & \\
\hline $\begin{array}{l}\text { Workload After } \\
\text { Companed to } \\
\text { Before the } \\
\text { Pandemic }\end{array}$ & & & & $<0.001$ & & & 0.937 \\
\hline Much Less & $\begin{array}{l}9 \\
{[6,87 \%]}\end{array}$ & $9(9.57 \%)$ & $\int_{(0,00 \%)}^{0}$ & & $\begin{array}{l}9 \\
{[7.56 \%)}\end{array}$ & $0(0.00 \%)$ & \\
\hline Somewhat less & $\begin{array}{l}39 \\
{[29.8 \%)}\end{array}$ & $\begin{array}{l}34 \\
(36.2 \%)\end{array}$ & $\begin{array}{l}5 \\
(13.5 \%)\end{array}$ & & $\begin{array}{l}36 \\
{[30.3 \%]}\end{array}$ & $3(25.0 \%)$ & \\
\hline Same & $\begin{array}{l}46 \\
\{35.1 \%\}\end{array}$ & $\begin{array}{l}34 \\
\mid 36.2 \%\}\end{array}$ & $\begin{array}{l}12 \\
(32.4 \%)\end{array}$ & & $\begin{array}{l}41 \\
\mid 34.5 \%\}\end{array}$ & $5(41.7 \%)$ & \\
\hline $\begin{array}{l}\text { Somewhat } \\
\text { more }\end{array}$ & $\begin{array}{l}27 \\
{[20.6 \%)}\end{array}$ & $\begin{array}{l}13 \\
(13.8 \%)\end{array}$ & $\begin{array}{l}14 \\
(37.8 \%)\end{array}$ & & $\begin{array}{l}24 \\
20.2 \%\end{array}$ & $3(25.0 \%)$ & \\
\hline Much more & $\begin{array}{l}10 \\
{[7,63 \%)}\end{array}$ & $4(4.26 \%)$ & $\int_{(16.2 \%)}^{6}$ & & $\begin{array}{l}9 \\
\text { [7.56\%) }\end{array}$ & $1(8.33 \%)$ & \\
\hline $\begin{array}{l}\text { Partner Lost a } \\
\text { Job }\end{array}$ & & & & 0.55 & & & 0.645 \\
\hline No & $\begin{array}{l}109 \\
(83,2 \%)\end{array}$ & $\begin{array}{l}80 \\
(85,1 \%)\end{array}$ & $\begin{array}{l}29 \\
(78,4 \%)\end{array}$ & & $\begin{array}{l}99 \\
{[83.2 \%)}\end{array}$ & $10(83.3 \%)$ & \\
\hline Yes. & $\begin{array}{l}6 \\
(4.58 \%)\end{array}$ & $4(4.26 \%)$ & $\left.\right|_{(5,41 \%)} ^{2}$ & & $\begin{array}{l}5 \\
(4.20 \%)\end{array}$ & $1(8.33 \%)$ & \\
\hline $\mathrm{N} / \mathrm{A}$ & $\begin{array}{l}16 \\
\{12.2 \%\}\end{array}$ & $\begin{array}{l}10 \\
\{10.6 \%\}\end{array}$ & $\begin{array}{l}6 \\
\{16.2 \%\}\end{array}$ & & $\begin{array}{l}15 \\
12.6 \%\end{array}$ & $1(8.33 \%)$ & \\
\hline $\begin{array}{l}\text { Pole in the } \\
\text { Department }\end{array}$ & & & & 0.021 & & & 0.024 \\
\hline Staff & $\begin{array}{l}86 \\
{[65.6 \%)}\end{array}$ & $\begin{array}{l}68 \\
|72.3 \%|\end{array}$ & $\begin{array}{l}18 \\
(48.6 \%) \\
\end{array}$ & & $\begin{array}{l}81 \\
(68.1 \%)\end{array}$ & $5(41.7 \%)$ & \\
\hline Fellow & $\begin{array}{l}4 \\
{[3,05 \%)}\end{array}$ & $3\{3.19 \%)$ & $\frac{1}{(2.70 \%)}$ & & $\begin{array}{l}2 \\
(1,68 \%)\end{array}$ & $2(167 \%)$ & \\
\hline $\begin{array}{l}\text { Advanced Care } \\
\text { Provider }\end{array}$ & $\begin{array}{l}41 \\
{[31,3 \%)}\end{array}$ & $\begin{array}{l}23 \\
(24.5 \%)\end{array}$ & $\begin{array}{l}18 \\
(48.6 \%)\end{array}$ & & $\begin{array}{l}36 \\
(30,3 \%)\end{array}$ & $5(41.7 \%)$ & \\
\hline Department & & & & 0.734 & & & 0,941 \\
\hline $\begin{array}{l}\text { Surgical Critical } \\
\text { Care }\end{array}$ & $\begin{array}{l}135 \\
\{26.7 \%\}\end{array}$ & $\begin{array}{l}23 \\
\{24.5 \%\}\end{array}$ & $\begin{array}{l}12 \\
(32.4 \%)\end{array}$ & & $\begin{array}{l}31 \\
\{26,1 \% 6\}\end{array}$ & $4(33.3 \%)$ & \\
\hline $\begin{array}{l}\text { Colorectal } \\
\text { Surgery }\end{array}$ & $\begin{array}{l}12 \\
{[9.16 \%)}\end{array}$ & $8(8.51 \%)$ & $\int_{(10.8 \%)}^{4}$ & & $\begin{array}{l}11 \\
{[9.24 \%]}\end{array}$ & $1(8.33 \%)$ & \\
\hline $\begin{array}{l}\text { Gastroenterolo } \\
\text { gy }\end{array}$ & $\begin{array}{l}32 \\
\{24.4 \%)\end{array}$ & $\begin{array}{l}24 \\
\{25.5 \%\}\end{array}$ & $\begin{array}{l}8 \\
\{21.6 \%)\end{array}$ & & $\begin{array}{l}30 \\
{[25.2 \%]}\end{array}$ & $2(16.7 \%)$ & \\
\hline $\begin{array}{l}\text { General } \\
\text { Surgery }\end{array}$ & $\begin{array}{l}5.2 \\
(39.7 \%)\end{array}$ & $\begin{array}{l}39 \\
\{41.5 \%\}\end{array}$ & $\begin{array}{l}13 \\
(35.1 \%)\end{array}$ & & $\begin{array}{l}47 \\
(39.5 \%)\end{array}$ & $5(41.7 \%)$ & \\
\hline
\end{tabular}

S026

\section{COVID-19 DISPROPORTIONATELY IMPACTED LOW INCOME AND MINORITY SURGICAL PATIENTS AT A LARGE QUATERNARY REFERRAL HOSPITAL}

Jennifer J Misenhimer, MD ${ }^{1}$; Steven Leeds, MD $^{1}$; Christine Sanchez, MPH $^{1}$; Katherine Litman, $\mathrm{BA}^{2}$; Gerald Ogola, $\mathrm{PhD}^{1}$; Marc Ward, $\mathrm{MD}^{1}$; ${ }^{1}$ Baylor University Medical Center; ${ }^{2}$ Texas A\&M College of Medicine.

Introduction: Coronavirus-19 (COVID) has stressed healthcare systems across the world by increasing hospital admissions and in-hospital mortality. The impact of this disease on patients undergoing elective or emergent surgery is unknown. The purpose of this study was to evaluate how the COVID- pandemic has affected surgical patients, case volume and surgical services in a large quaternary referral hospital. Methods: A retrospective review of patients undergoing surgical procedures was performed to evaluate patient demographics, payor information, patient outcomes, surgical case volume, and surgical case type. These areas of interest were divided into four timeframes that were roughly based off government regulations and lockdown: Pre COVID (1/1/20- 3/15/20), Suspended (3/16/20-5/4/20), Backlogged Cases (5/5/20-6/14/20) and Restoration Phase (6/15/20-9/30/20). Differences in patient characteristics and outcomes were compared between the four timeframes using Chi-square for categorical variables, and analysis of variance (ANOVA) for continuous variables. Locally weighted least squares (LOESS) regression was used to fit a smoothing curve to assess weekly trend in surgery volume over time.

Results: A total of 21,424 surgical cases were performed between 1/1/20 and 9/30/ 20. The Suspended time frame (when all elective cases were cancelled) had the lowest number of cases, $2,238(10.4 \%$; $<<0.01)$. During this time, the percentage of males undergoing surgery increased $(53.8 \%$ compared to $49.2 \%$ Pre COVID; $p<$ 0.01). In addition, the number of black and Hispanic surgical patients increased compared to all other time points $(23.1 \%$ and $15.3 \%$, respectively; $\mathrm{p}<0.05)$ Medicaid and self-pay patients increased from $9.9 \%$ of all surgical patients Pre COVID to $16.3 \%$ during the Suspended timeframe $(\mathrm{p}<0.01)$ and remained elevated at all other time points $(13.1 \%$ and $11.6 \%$ respectively). Restoration Phase Cases experienced the largest number of cases overall $(9,177 ; \mathrm{p}<0.01)$. The overall number of cases performed each week can be seen in Fig. 1. All service lines experienced similar trends to the overall case volume except obstetrics (constant) and trauma (which increased during the Suspended timeframe).

Conclusions: COVID-19 has disproportionately increased the number of low income and minority surgical patients treated at a large Quaternary Referral Hospital. Overall surgical volume has increased compared to pre-pandemic levels.

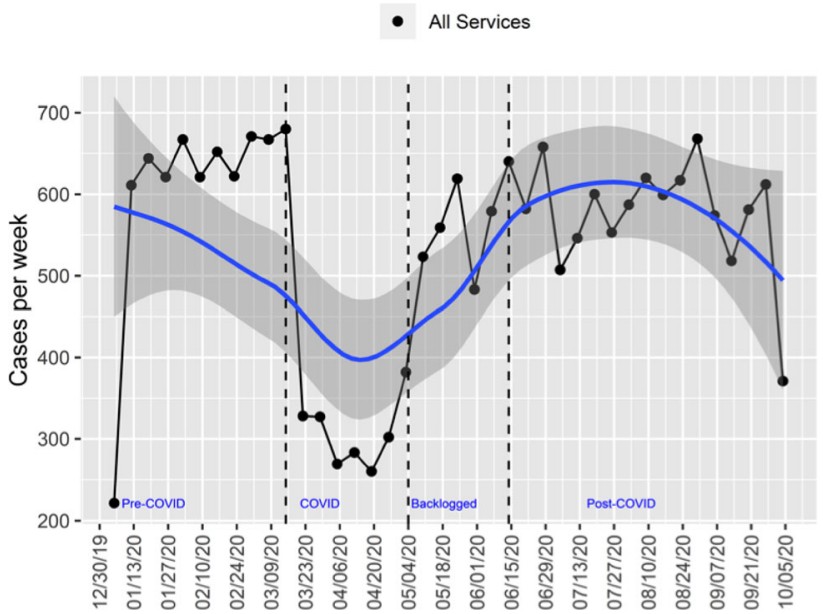


S027

\section{THE IMPACT OF THE NOVEL CORONAVIRUS PANDEMIC ON GASTROINTESTINAL OPERATIONS IN THE UNITED STATES}

Amanda C Purdy, $\mathrm{MD}^{1}$; Brian R Smith, $\mathrm{MD}^{2}$; Samuel F Hohmann, $\mathrm{PhD}^{3}$; Ninh T Nguyen ${ }^{2} ;{ }^{1}$ Harbor UCLA Medical Center; ${ }^{2}$ University of California, Irvine; ${ }^{3}$ Rush University.

INTRODUCTION: In March 2020, the American College of Surgeons recommended the limitation of elective procedures to prepare for the COVID-19 surge. We hypothesize one of the consequences of the pandemic is a decrease in operative volume across the country. The objective of this study is to examine changes in volume of common gastrointestinal operations during the COVID-19 pandemic, including elective, urgent/emergent, and cancer operations. We also aim to determine if hospitals with the most COVID-19 admissions were most impacted.

METHODS AND PROCEDURES: The Vizient database was queried using ICD10 codes to determine the number of patients per month from November 2019 through June 2020 who underwent each of the following operations: elective hiatal hernia repair and bariatric surgery; urgent cholecystectomy, appendectomy, and inguinal hernia repair; and colectomy, gastrectomy, and esophagectomy for cancer. The database was also used to determine COVID-19 admissions per hospital. November 2019 through January 2020 was defined as the "pre-COVID" group. The percent reduction in volume from pre-COVID was calculated for each operation by month. The $25 \%$ of hospitals with the most COVID cases were also evaluated separately from hospitals with fewer COVID cases. Analysis of variance was used to determine if there was a significant difference in case volume by month.

RESULTS: Data from 559 hospitals were analyzed. The volume of all operations evaluated in this study were significantly reduced during the pandemic except gastrectomies and esophagectomies for cancer. The greatest reduction in all operations was in April (24-98\% reduction). In April, the volume of bariatric surgery reduced by $98 \%(\mathrm{P}<0.001)$, hiatal hernia repairs by $96 \%(\mathrm{P}<0.001)$, urgent cholecystectomies by $42 \%(\mathrm{P}<0.001)$, urgent appendectomies by $24 \%(\mathrm{P}<0.001)$, urgent inguinal hernia repairs by $40 \%(\mathrm{P}<0.001)$, and colectomies for cancer by $39 \%(\mathrm{P}<$ 0.001 ). The $25 \%$ of hospitals with the most COVID-19 admissions had a greater reduction in all cases than hospitals with fewer COVID cases. There was a rebound in performance of all operations by June 2020 (6-17\% reduction).

CONCLUSIONS: The coronavirus pandemic led to a significant reduction in volume of all gastrointestinal operations evaluated except gastrectomies and esophagectomies for cancer. The greatest reduction was in April 2020, with a rebound of case volume by June 2020. Hospitals with the most COVID-19 admissions were most impacted. While elective, non-cancer operations were the most affected, urgent and some cancer operations also saw significant volume reductions. As COVID-19 continues to surge, Americans may suffer continued limited access to surgical care.

\section{S028}

\section{RESIDENT CASELOAD AND OPERATIVE VOLUME IN THE TIME OF CORONAVIRUS}

Stephen A Iacono, MD, MPH; Rachel E Nemoyer, MD, MPH; Michael T Scott, MD, MPH; Nell Maloney Patel, MD; Stanley Z Trooskin, MD; Robert Wood Johnson Medical School.

INTRODUCTION: The novel Coronavirus pandemic had an irrevocable impact on surgical education inside and outside of the operating room (OR). Elective caseload halted during the height of the pandemic causing a decrease in case volume for residents, particularly graduating seniors. While the pandemic led to elective surgery cessation, the need for urgent and emergent surgeries continued. The purpose of this study was to investigate the impact of COVID-19 on resident operative case volume compared to prior years and discuss the potential impact on overall surgical education.

METHODS: A retrospective review of all OR cases and resident case logs at a large tertiary care center between $3 / 22 / 2020$ and $6 / 1 / 2020$ were reviewed. The OR cases and case logs in 2020 were compared to the same dates in 2018 and 2019. Case volumes were stratified by services covered by General Surgery residents. Trauma activations for the same months were also reviewed. Statistical analysis was done using analysis of variance (ANOVA) at a significance level of $\mathrm{p}=0.05$.

RESULTS: In 2018 and 2019 there were 1,609 and 1,759 OR cases eligible for resident coverage. OR cases decreased significantly in 2020 to 1,015 cases ( $\mathrm{p}<$ 0.05 ). The decrease in case volume between years 2018 and 2019 compared to 2020 was significant in almost all specialties including general surgery (426 vs 390 vs 151 ; $\mathrm{p}=0.002)$, cardiothoracic surgery ( 349 vs 422 vs $199 ; \mathrm{p}=0.002)$, pediatric surgery ( 187 vs 214 vs $147 ; \mathrm{p}=0.05$ ), and vascular surgery ( 224 vs 296 vs $114 ; \mathrm{p}=0.01$ ). There was no significant difference in case volume for surgical oncology (204 vs 192 vs $185 ; \mathrm{p}=0.902$ ) or acute care surgery ( 218 vs 203 vs $208 ; \mathrm{p}=0.730$ ). There was also no significant difference in number of trauma activations in 2020 compared to 2018 and 2019 ( 329 vs 400 vs 421 respectively; $p=0.11$ ). Amongst surgical residents, there were significantly lower cases logged per resident $(\mathrm{p}<0.05)$ in Postgraduate Year (PGY)-2 (49.5 vs 61.4 vs 15.5 ), PGY-3 (47.5 vs 52.0 vs 18.2 ), and PGY-5 (51.5 vs 47.5 vs 18.2). Cases logged by PGY-1 (23.6 vs 32.2 vs 15.3 ; p = $0.11)$ and PGY-4 (54.8 vs 46.0 vs $30.0 ; \mathrm{p}=0.10$ ) were not significantly lower than previous years.

CONCLUSION: The COVID-19 pandemic had a significant decrease on operative experience during the initial surge. The overall effects on surgical education and operative experience remains to be seen as OR volumes have increased over recent months, but possibility of resurgence continues to loom, threatening surgical resident education. 
S029

\section{IMPACT OF COVID-19 PANDEMIC ON REVENUE GENERATED BY EMERGENCY GENERAL SURGERY PROCEDURES}

Aman Ali, MD; Asad Shaikh, MD; Mahnoor Zia; Nima Maghami, MD, FACS; Daniel Bonville, DO, FACS; Houston Methodist Hospital.

Introduction: The COVID-19 pandemic has changed the dynamics of healthcare in the United States. In early 2020, most states issued orders to stop non-emergent elective surgeries. This contracted the overall revenue generated by the hospital systems. However, the impact of COVID-19 pandemic on revenue generated emergent cases remains unexplored. The goal of this study was to assess if COVID19 pandemic has affected revenues from emergency general surgeries.

Methods: This is a retrospective review to compare surgical case volume in 2019 and 2020. We obtained our data from a tertiary care referral center database. Data was collected from February to April of 2019 and 2020, corresponding to the duration of statewide ban on non-emergent surgical cases. We used the most reported current procedural terminology (CPT) Code for each surgical procedure to calculate the revenue generated. We calculated the percentage difference in revenue between 2020 and 2019 for comparison.

Results: Results for our study are shown in Table 1. We observed a drop in the total number of emergency surgeries between February to April of 2020 compared to the surgical volume during same time frame in 2019. There is a decline in revenue generated in 2020 compared to 2019 for all emergent surgeries.

Conclusion: The COVID-19 pandemic has negatively impacted surgical case volumes in 2020 compared to 2019. This includes both emergent and non-emergent cases. There is a need for more broad cost analysis which considers hospital expenditures and cost benefit analysis.

Table 1: Revenue generated by emergent general surgery cases in 2019 and 2020

\begin{tabular}{|l|c|c|c|c|c|}
\hline Procedure & $\begin{array}{c}\text { \# of Cases } \\
2019\end{array}$ & $\begin{array}{c}\text { \# of Cases } \\
2020\end{array}$ & $\begin{array}{c}\text { Revenue in } \\
2019(\$)\end{array}$ & $\begin{array}{c}\text { Revenue in } \\
2020(\$)\end{array}$ & $\begin{array}{c}\text { Revenue } \\
\text { Lost (\%) }\end{array}$ \\
\hline Appendectomy & 318 & 242 & 127719.61 & 97195.43 & -23.90 \\
\hline Cholecystectomy & 678 & 425 & 268968.23 & 168601.03 & -37.32 \\
\hline Sigmoid Colectomy with colostomy & 37 & 29 & 38959.63 & 30535.93 & -21.62 \\
\hline $\begin{array}{l}\text { Sigmoid colectomy with } \\
\text { anastomosis }\end{array}$ & 201 & 109 & 258864.10 & 140379.04 & -45.77 \\
\hline Skin \& Soft Tissue Infections (SSTI) & 180 & 133 & 73658.16 & 54425.20 & -26.11 \\
\hline Enterolysis & 29 & 14 & 20284.03 & 9792.29 & -51.72 \\
\hline Resection of Small Intestine & 35 & 17 & 27610.44 & 13410.79 & -51.43 \\
\hline Endoscopic procedure for bleeding & 51 & 31 & 13565.38 & 8245.62 & -39.22 \\
\hline Incarcerated Hernia & 84 & 34 & 47550.43 & 19246.60 & -59.52 \\
\hline Reducible hernia & 564 & 181 & 254729.92 & 81748.43 & -67.91 \\
\hline
\end{tabular}

\section{S030}

Impact of the Covid-19 pandemic on rates emergency department utilization due to general surgery conditions

Saba Balvardi, MD; Josie Cipolla, RD; Pepa Kaneva, MSc; Sebastian Demyttenaere, MD; Marylise Boutros, MD; Lawrence Lee, MD; Liane Feldman, MD; Julio Fiore Junior; McGill University.

Background: Recent literature reports a decrease in healthcare-seeking behaviors by adults during the COVID-19 pandemic. Given that emergency general surgery (GS) conditions are often associated with high risk of morbidity and mortality if left untreated, there is a need to characterize emergency department (ED) utilization and GS management during the pandemic. The objective of this study is to quantify the impact of the Covid-19 pandemic on rates of emergency department utilization due to GS conditions.

Methods and Procedures: This study involved the analysis of an institutional database. We identified adult patients presenting to the ED in a network of three teaching hospitals in Montreal, Canada during the early stages of the COVID-19 pandemic (March13-May13, 2020) and a control pre-pandemic period (March13May13, 2019). Patients with GS conditions (based on AAST list of emergency GS pathologies) were included in the analysis. Comparison between the two time periods was conducted using Chi-square and Poisson exact test.

Results: During the pre-pandemic period, 352 patients presented to ED with a confirmed GS diagnosis compared to 259 patients during the pandemic (26\% reduction, $\mathrm{p}<0.001)$. There was a significant reduction in the number of patients presenting with appendicitis, pancreatitis, bowel obstruction and colorectal diseases (perianal disease, colitis, diverticulitis and colon cancer) during the pandemic period ( $p<0.05$, Figure). There was no significant difference in the number of patients presenting with biliary disease and perforated viscus $(\mathrm{p}>0.05)$. Overall, 111 patients were managed operatively in the pre-pandemic period compared to 80 patients during the pandemic period ( $28 \%$ reduction, $\mathrm{p}=0.001$ ). However, in relation to the total number of presentations, rates of surgical management of common GS conditions were not significantly different: appendectomies [38/50 (76\%) pre-pandemic vs. 37/26 (70\%) during the pandemic; $\mathrm{p}=0.63]$ and cholecystectomies $[22 / 35(63 \%)$ pre-pandemic vs. $18 / 33(54 \%)$ during the pandemic; $p=0.65]$. Of 140 patients $(55 \%)$ tested for COVID-19 during the pandemic period, 2 tested positive.

Conclusion: Our findings suggest a decrease in the health-seeking behavior of adults with GS conditions in early stages of the COVID-19 pandemic. The impact of potential delays in ED presentations on patient outcomes requires further investigations. Rates of operative management of common GS conditions were unchanged during the pandemic, suggesting that there was no prioritization for conservative management.

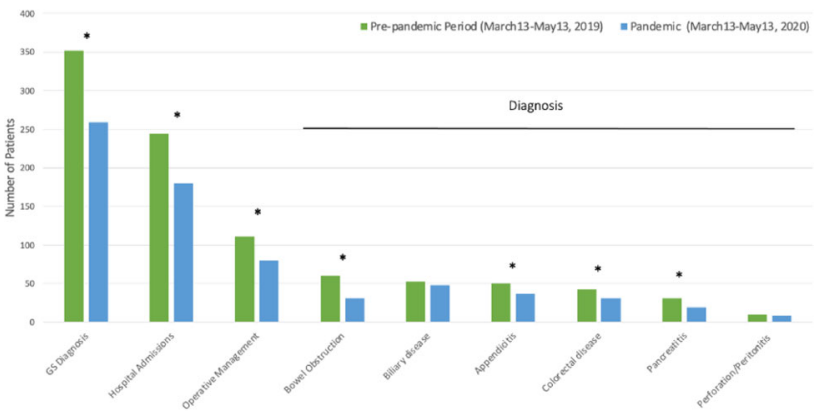

Figure: Emergency department utilization due to general surgery conditions (* indicate $\mathrm{p}<0.05)$. 
S031

\section{Age and BMI Are Poor Predictors of Infectious Outcomes in Older Hernia Patients following Transversus Abdominis Release}

Bradley S Kushner, MD; Julia Hamilton, BS; Britta Han, MD; Jeffrey Blatnik, MD, FACS; Timothy Holden, MD; Sara E Holden, MD; Washington University in St. Louis.

Introduction: Transversus Abdominis Release (TAR) is an effective procedure for the repair of complex ventral hernias. However, TARs are not low risk operations, particularly in older adults who are disproportionately affected by multiple agerelated risk factors. While past studies have suggested that age alone inconsistently predicts overall patient outcomes, data regarding age's effect specifically on infectious outcomes following a TAR is lacking. Additionally, while body mass index (BMI) is a known risk factor for infections after a TAR, whether BMI in older adults also predicts infectious outcomes is unknown.

Methods: This was a retrospective cohort study of patients undergoing an open or robotic TAR by two fellowship-trained abdominal wall specialists from 1/2018 to $2 / 2020$. Patients were stratified by age $(\geq 60$ years of age $(\mathrm{O})$ or $<60$ years $(\mathrm{Y})$ ) and by both age and BMI $(<30,30-35$, and $>35)$. Demographic information, perioperative clinical data (operating time (OT-minutes), length of stay (LOS-days), and hernia defect size $(\mathrm{cm} 2)$ ), and infectious complications (surgical site occurrence (SSO) at 30 days, surgical site infection (SSI), abdominal wall/deep abscess, seromas/hematomas, cellulitis, surgical site occurrence requiring procedural intervention (SSOPI), antibiotic prescription, and 30-day readmission rate) were analyzed. Patients undergoing combined procedures or biologic mesh-based repairs were excluded. Mann-Whitney U test was used to analyze continuous data and the ChiSquare test for categorical data.

Results: There were 211 patients eligible for inclusion (117 patients were $\geq$ 60 years). Cohorts stratified by age were well-matched (defect size $(p=0.6)$, BMI ( $=0.4)$, OT $(\mathrm{p}=0.5), \operatorname{LOS}(\mathrm{p}=0.4)$, and percent open procedures $(\mathrm{p}=0.3))$ as were those stratified by age and BMI (defect size: $\mathrm{p}=0.12$; age: 0.55 ; OT: 0.22 ; and LOS: $0.50)$. When stratified by age alone, there were no differences in SSO $(\mathrm{p}=0.5)$, SSI $(\mathrm{p}=0.07)$, seromas/hematomas $(\mathrm{p}=0.6)$, cellulitis $(\mathrm{p}=0.08)$, antibiotics $(\mathrm{p}=0.08)$, or readmissions $(\mathrm{p}=0.5)$. In fact, $<60$ had greater rates of abscesses $(\mathrm{p}=0.02)$ and SSOPI $(\mathrm{p}=0.02)$. Only seromas/hematomas differed in older adults stratified by $\mathrm{BMI}$ (higher in BMI $>35 ; \mathrm{p}=0.03$ ). In comparison, higher BMI was predictive of infectious outcomes in younger adults: SSO $(p=0.01)$, abscess $(p=0.01)$, cellulitis $(\mathrm{p}<0.01)$, antibiotics $(\mathrm{p}<0.01)$, and SSOPI $(\mathrm{p}=0.02)$ and a trend towards higher SSI rates $(\mathrm{p}=0.08)$.

Conclusion: In older adults, age and BMI are poor predictors of infectious outcomes following a TAR. Our findings suggest that obesity in older adults is not an identical risk-factor to that in younger patients. Further investigation of alternative preoperative screening tools that better predict age-related risk factors are needed to optimize outcomes in older adults.

\section{S032}

\section{Outcomes from Laparoscopic versus Robotic Mesh Removal after Inguinal Hernia Repair}

Desmond T Huynh, MD ${ }^{1}$; Xiaoxi Feng, MD ${ }^{1}$; Negin Fadaee, $\mathrm{BS}^{2}$; Nicholas Gonsalves, MD ${ }^{1}$; Shirin Towfigh, MD, FACS ${ }^{2} ;{ }^{1}$ Cedars-Sinai Medical Center; ${ }^{2}$ Beverly Hills Hernia Center.

INTRODUCTION: Retroperitoneally placed mesh for inguinal hernia repair may require removal to address hernia recurrence, mesh reaction, meshoma, or other chronic pain. These are best approached either laparoscopically or robotically, but there is no consensus on which is the best approach for mesh removal nor are there any studies to evaluate and compare their outcomes.

METHODS AND MATERIALS: All patients who underwent inguinal mesh removal via laparoscopic and robotic approaches from 2011 to 2020 were analyzed. Data regarding demographics, pre-operative, intraoperative, and post-operative outcomes were collected.

RESULTS: Over 9 years, 62 patients underwent 24 laparoscopic and 50 robotic approaches to remove 88 inguinal meshes. There were no significant differences in the two groups for age, sex, or comorbidities. The laparoscopic group had a significantly lower BMI $(23.5$ vs $26.7 \mathrm{~kg} / \mathrm{m} 2, \mathrm{p}=0.006)$. The majority $(60 \%)$ of patients were performed as an outpatient. There were no significant differences in surgical site occurrences or hospital length of stay.

Patients in both groups showed significant improvement after mesh removal with pain score reduction of 1.6 and 1.7 points $(\mathrm{p}=0.02, \mathrm{p}<0.01)$ within 2 weeks postoperatively and 2.3 and 1.8 points $(\mathrm{p}<0.01, \mathrm{p}<0.01)$ at mean long term followup of 18 months (range 2 weeks-62 months).

Table 1: Outcomes of laparoscopic versus robotic mesh removal. Continuous data presented as
mean [range]
\begin{tabular}{|l|c|c|c|} 
& Laparoscopic & Robotic & p \\
\hline Number of inguinal mesh removed & 29 & 59 & \\
\hline & & & \\
\hline Preoperative pain score, 0-10 & $6.2[2-10]$ & $5.6[0-10]$ & NS \\
\hline Two week followup pain score, 0-10 & $4.4[0-10]$ & $4.0[0-10]$ & NS \\
\hline Long-term follow-up (LTF) pain score, 0-10 & $3.6[1-9]$ & $4.3[0-9]$ & NS \\
\hline Pain score reduction at LTF & $2.3[-2-9]$ & $1.8[-5-10]$ & NS \\
\hline & & & \\
\hline Operative time per mesh removed, minutes & $162[35-363]$ & $217[64-420]$ & 0.02 \\
\hline Estimated blood loss per mesh removed, mI & $86[0-700]$ & $57[5-209]$ & NS \\
\hline Intraoperative injury & $8(28 \%)$ & $8(14 \%)$ & NS \\
\hline Vascular & $7(24 \%)$ & $7(12 \%)$ & NS \\
\hline Gonadal artery & $0(0 \%)$ & $1(2 \%)$ & NS \\
\hline Inferior epigastric artery & $6(20 \%)$ & $5(8 \%)$ & NS \\
\hline Major vascular (external iliac) & $3(10 \%)$ & $1(2 \%)$ & NS \\
\hline Nerve & $1(3 \%)$ & $1(2 \%)$ & NS \\
\hline Organ & $0(0 \%)$ & $0(0 \%)$ & NS
\end{tabular}

CONCLUSION: It is our experience that both laparoscopic and robotic approaches are effective for removal of retroperitoneally placed inguinal mesh. Operative time with the laparoscopic approach was significantly shorter than the robotic approach, by about an hour. Our patients on average had significant reduction in their preoperative pain, regardless of the approach.

We present the largest study to evaluate retroperitoneal inguinal mesh removal for both laparoscopic and robotic approaches. These are technically challenging operations with risk of vascular and nerve injuries regardless of the approach. We note a trend toward higher risk of major vascular injury when performed laparoscopically $(10 \%$ vs $2 \%)$. In our practice, we prefer the robotic approach for inguinal mesh removal, primarily because it provides us with more effective handling of the intrinsic risks associated with this operation, especially with vascular injuries. 
S033

\section{The Role of Insurance in Postoperative Outcomes and Quality of Life Following Ventral Hernia Repair}

Savannah M Renshaw, BS; Rosevine A Azap, BS; Anand Gupta, MBBS, MPH; Courtney E Collins, MD, MS; The Ohio State University Wexner Medical Center.

Background: Access to care and barriers to achieving health equity remain persistent and prevailing issues in the U.S. Insurance is often regarded as a surrogate marker of socioeconomic status, and previous studies have identified insurance type as a predictor of emergent repair in a variety of surgical specialties. However the impact of insurance type on postoperative outcomes following ventral hernia repair (VHR) remains unknown.

Methods: The 2013-2020 Abdominal Core Health Quality Collaborative (ACHQC) database was used to identify patients aged 18-64 undergoing ventral hernia repair (VHR) who had private or Medicaid insurance. Patients with no health insurance were also included. Using insurance type, the cohort was divided into 3 groups: private, public (Medicaid), and uninsured (self-pay). Multivariate logistic regression analyses were used to assess the impact of insurance type on 30-day postoperative outcomes, including complications, emergency department (ED) visits, readmission, reoperation, and changes in quality of life from baseline to 1-year using the HerniaRelated Quality of Life Survey (HerQLes).

Results: A total of 17,036 patients undergoing VHR were included in the study, out of which $13,980(85.8 \%)$ had private insurance, $2,451(8.4 \%)$ had public, and 605 $(5.8 \%)$ were uninsured. Following adjustment for demographics (age, gender, race), comorbidities (hypertension, diabetes, smoking), and clinical characteristics (emergent, ASA class, approach), having public insurance or being uninsured was associated with increased odds of experiencing postoperative complications, compared to those who were privately insured (public: OR $1.3, \mathrm{p}<0.01$; self-pay: OR $1.67, \mathrm{p}<0.01$ ). Baseline quality of life was significantly different among groups, with publicly insured and self-pay patients presenting with -7.64 points lower and 5.72 points lower, respectively (public: $95 \%$ CI: $-9.99,-5.28$; self-pay: $95 \%$ CI: $10.69,-0.75 ; \mathrm{p}<0.01)$.

Conclusions: Our study demonstrates that public and self-pay insurance are associated with worse postoperative outcomes compared to those with private insurance. In an effort to promote health equity, healthcare providers need to assess how parameters beyond physical presentation may impact a patient's health.

Table 1 Univariate Analysis of 30-Day Postoperative Outcomes by Payer Type

\begin{tabular}{lllll}
\hline & Private & Public & Uninsured & p-value \\
\hline Complication & $1573(12.8 \%)$ & $391(18.8 \%)$ & $95(19.8 \%)$ & $<0.01$ \\
ED Visit & $454(3.2 \%)$ & $186(7.6 \%)$ & $38(6.3 \%)$ & $<0.01$ \\
Readmission & $396(3.2 \%)$ & $135(6.5 \%)$ & $30(6.3 \%)$ & $<0.01$ \\
Reoperation & $122(1.0 \%)$ & $47(2.3 \%)$ & $8(1.7 \%)$ & $<0.01$ \\
\hline
\end{tabular}

\section{S034}

Multicenter Analysis of Laparoscopic Versus Open Umbilical Hernia Repair with Mesh: Outcomes and Quality of Life (QOL)

Sharbel Elhage, MD; Sullivan Ayuso, MD; Jenny Shao, MD; Eva Deerenberg, MD, PhD; Tanu Prasad; Paul Colavita, MD; Vedra Augenstein, MD; B Todd Heniford, MD; Carolinas Medical Center.

Introduction: Umbilical hernia repair (UHR) is a common operation with varying surgical approaches. Laparoscopic (LUHR) and open (OUHR) operations are routinely performed, but their impact on QOL is not well described. The aim of this study was to compare the outcomes and quality of life (QOL) of LUHR versus OUHR.

Methods: A prospectively collected international multicenter hernia database was queried for patients undergoing UHR with mesh placement. Carolinas Comfort Scale was used for QOL assessment, and addresses pain, mesh sensation, activity limitation, and overall QOL. QOL was measured preoperatively and at 1, 6, 12, and 24 months postoperatively. Propensity match was performed controlling for hernia defect area (HDA), recurrent hernias, and BMI.

Results: In total there were 585 patients who underwent 178 (30.4\%) LUHR and $407(69.6 \%)$ OUHR. LUHR patients had higher BMI, larger HDA, and were more often recurrent (all $\mathrm{p}<0.05$ ). Rate of comorbidities (cardiac disease, tobacco use, diabetes mellitus, immunosuppression) were similar $(\mathrm{p}>0.05)$. In LUHR, fascial closure was achieved in $24.2 \%$ of patients. Tacks were used more frequently in LUHR $(92.1 \%$ vs $2.2 \%, \mathrm{p}<0.001)$, and suture was used more often in OUHR $(97.5 \% \mathrm{vs} 48.4 \%, \mathrm{p}<0.001)$. Hematoma, infection, and recurrence rates (LUHR8.4\%vsOUHR4.7\%; follow-up $19.9 \pm 11.0$ months) were all similar (all p > $0.05)$. LUHR had higher rates of seroma postoperatively $(13.9 \% \mathrm{vs} 4.3 \%, \mathrm{p}<0.001)$. Preoperative QOL was similar overall in each group, and similar in pain and mesh sensation for those with prior mesh placement. Postoperatively, QOL data were available for an average of $457 \pm 67$ patients $(78.1 \%)$ at each time period. QOL was superior in the OUHR group in all four QOL variables at 1 and 24 months (all p < 0.05 ), with less pain and reduced mesh sensation at 6 and 12 months (all $\mathrm{p}<0.05$ ). Fascial closure did not impact LUHR QOL $(\mathrm{p}>0.05)$. Given the possible effects of difference in BMI, recurrent hernias, and HDA, a propensity match was performed yielding 138 matched pairs. The seroma rate remained higher in the LUHR group $(12.5 \% \mathrm{vs} 4.4 \%, \mathrm{p}=0.01)$, and OUHR had a higher rate of infection $(0.0 \% \mathrm{vs} 3.7 \%$, p $=0.02$ ). Hernia recurrence was higher in the LUHR group at $9.4 \%$ vs $2.9 \%$ (p = 0.02 ). QOL evaluation showed preoperative pain was similar, but the OUHR group had improved postoperative pain assessments at one $(\mathrm{p}=0.03)$ and six $(\mathrm{p}=0.02)$ months.

Conclusion: OUHR is associated with higher rates of surgical site infections, but significantly lower rates of seroma formation and recurrence compared to LUHR. QOL was also superior in the OUHR group in both short and long-term follow-up. 


\section{S035}

\section{Geriatric Assessment and Medical Preoperative Screening (GrAMPS) Program for Older Hernia Patients}

Bradley S Kushner, MD; Britta Han, MD; Margaret Sehnert, BS, RHIA; Timothy Holden, MD; Sara E Holden, MD; Washington University in St. Louis.

Introduction: The incidence of inguinal and ventral hernia repairs in older adults is increasing as the population ages. These hernia repairs should not be considered low risk operations, particularly in older adults who are disproportionately affected by multiple age-related factors (multimorbidity, frailty, cognitive impairment, depression, obesity, functional impairment, polypharmacy, and poor subjective health). These age-related risk factors adversely affect surgical outcomes and have been shown to increase patient morbidity by three-fold. We present the results of phase one of our multi-phase study aimed at identifying the prevalence of age-related risk factors in our elective hernia population.

Methods: Patients over the age of 60 with a planned surgical repair of a ventral or inguinal hernia were prospectively enrolled at our academic medical center's comprehensive hernia clinic. In addition to collecting demographic and clinical data, enrolled subjects completed several validated screening tools including the MiniCog, subjective memory concern, FRAIL-Scale, PHQ-2, self-reported health assessment, Lawton-Brody Instrumental Activities of Daily Living Scale, and the Katz Index of Independence in Activities of Daily living. Polypharmacy was defined as five or more medications, and multimorbidity as 3 or greater on the Charlson Comorbidity Index.

Results: Sixty-eight (68) of 72 eligible patients completed our preoperative assessments with an average age and BMI of 68.5 years and $28.1 \mathrm{~kg} / \mathrm{m} 2$, respectively. The majority were male $(67.6 \%)$. Twenty-four patients $(35.2 \%)$ had a BMI over 30 (obese). In total, $36.8 \%$ screened positive for objective cognitive impairment (mini-cog) and $47.1 \%$ for a subjective memory concern. Patients were on an average of 10.8 medications and, all but 9 patients $(86.8 \%)$ met criteria for polypharmacy. Additionally, $72.5 \%$ screened positive for either pre-frailty $(54.1 \%)$ or frailty $(17.6 \%)$ and $94.1 \%$ had multi morbidity. A total of $36.8 \%$ of patients self-rated their health as "poor" or "fair", and $17.6 \%$ screened positive for depression. Lastly, $13.2 \%$ endorsed some degree of objective functional impairment.

Conclusions: There is a high prevalence of often unrecognized and underappreciated age-related risk factors in patients undergoing elective ventral or inguinal hernia repair. These age-related risk factors may be associated with adverse surgical outcomes, significantly impact patient's ability to offer informed consent, and actively participate in their treatment plan. Additional study is required to integrate these agerelated risk factors into the preoperative assessment, which will subsequently inform shared-decision making and individualize risk factor modification to improve patient-centered and surgical outcomes.

\section{S036}

\section{Improved Hernia Variable Communication Across A Statewide Collaborative; A Pilot Study}

Lia D Delaney, $\mathrm{BS}^{1}$; Ryan Howard, $\mathrm{MD}^{2}$; Rhonda Rogers, BNS, $\mathrm{RN}^{2}$; Anne P Ehlers, MD, $\mathrm{MPH}^{2}$; Michael Englesbe, $\mathrm{MD}^{2}$; Dana Telem, MD, $\mathrm{MPH}^{2}$; ${ }^{1}$ University of Michigan Medical School; ${ }^{2}$ University of Michigan.

Introduction: Variable approaches to intraoperative communication and documentation impede our understanding of surgical decision-making and best practices. This is especially critical among hernia repairs, where improved patient outcomes are reliant on understanding the impact of variable patients, operative timepoints, and surgical approaches. In an effort to improve the care of hernia patients, we aimed to increase and standardize intraoperative communication and documentation among hernia repairs. In this context, a synoptic operative note was piloted prior to statewide dissemination in order to understand the impact of the intervention.

Methods: The Michigan Surgical Quality Collaborative (MSQC) is 70 hospital statewide collaborative with a registry that captures patient and postoperative variables. In January 2020, the registry was expanded to capture hernia-specific intraoperative variables. Eight hospitals piloted a synoptic operative note for hernia repair. A prospective quality improvement study assessed the impact of the synoptic operative note on intraoperative data collection. The primary outcome was a change in communication of hernia-specific variables, measured by completeness in the MSQC registry. Variable missingness was compared between pilot and non-pilot sites.

Results: From January to June 2020, 870 hernia repairs were performed, with 157 $(18 \%)$ at 8 pilot sites and $713(82 \%)$ at 53 non-pilot sites. Mean (SD) age was $55(15)$ years old and $461(53 \%)$ patients were male. Pilot sites had significantly less missingness for all hernia-specific variables (Table 1). 95 (46\%) of pilot site cases, and $228(21 \%)$ of non-pilot site cases, had no missing variables.

Conclusion: Six months after implementation, the synoptic operative note was associated with increased documentation and communication. Upscaling synoptic operative note utilization can standardize communication across the state to provide better quality of care for patients. Further work will focus on a more nuanced understanding of barriers and facilitators to implementation.

Table 1. Comparison of Intraoperative Variable Missingness

\begin{tabular}{lllr}
\hline & $\begin{array}{l}\text { Pilot Sites } \\
(\mathbf{n = 8})\end{array}$ & $\begin{array}{l}\text { Non-pilot Sites } \\
(\mathbf{n = 5 3 )}\end{array}$ & P-value \\
\hline Hernia Location & $2 \%$ & $10 \%$ & 0.001 \\
Recurrent Hernia & $0 \%$ & $7 \%$ & 0.001 \\
Hernia Length & $29 \%$ & $38 \%$ & 0.032 \\
Hernia Width & $49 \%$ & $68 \%$ & $<0.001$ \\
Mesh Used: Yes/No & $1 \%$ & $4 \%$ & 0.056 \\
Mesh Type & $2 \%$ & $21 \%$ & $<0.001$ \\
Component Separation & $4 \%$ & $35 \%$ & $<001$ \\
\hline
\end{tabular}


S038

\section{Evaluating the Risk of Surgical Neurectomies}

Xiaoxi Feng, MD, MPH ${ }^{1}$; Negin Fadaee, $\mathrm{BS}^{2}$; Desmond Huynh, $\mathrm{MD}^{1}$; Isabel Capati, $\overline{\mathrm{RN}}, \mathrm{BSN}^{2}$; Shirin Towfigh, $\mathrm{MD}^{2} ;{ }^{1}$ Cedars Sinai Medical Center; ${ }^{2}$ Beverly Hills Hernia Center.

INTRODUCTION: Neuralgia due to a peripheral nerve injury may result in chronic pain, requiring a therapeutic surgical neurectomy. In contrast, some neurectomies are performed prophylactically, such as during open inguinal hernia repair or inguinal mesh removal. Outcomes after neurectomy are largely unknown despite a common sentiment that it is a benign procedure with little to no risk.

METHODS: All patients who underwent neurectomy between 2013-2020 were analyzed. Data collection included demographics, pre-operative symptoms, and postoperative outcomes. Indication for neurectomy is categorized as "therapeutic" if the patient had preoperative neuralgia or "prophylactic" if deemed necessary intraoperatively without preoperative neuralgia. Statistical analysis included MannWhitney U test and paired Wilcoxon signed-rank test.

RESULTS: 66 patients underwent 78 operations with a total 119 neurectomies, with a median of one neurectomy per operation. $37(56 \%)$ patients were male with average age of 50 years (range 21-80). $53(44 \%)$ neurectomies were therapeutic, versus $66(56 \%)$ prophylactic. The transected nerves included ilioinguinal $(51,43 \%)$, iliohypogastric $(29,24 \%)$, genitofemoral $(32,27 \%)$, cutaneous $(5,4 \%)$, and intercostal $(2,2 \%)$. Among therapeutic neurectomies, $11(21 \%)$ proved to have a neuroma on pathology.

Average pre-operative pain score was 5.8/10 (SD 2.4); two-week post-operative pain score was 4.0/10 (SD 3.1) Patients in the prophylactic group had a significant postoperative pain reduction of 1.8 points $(\mathrm{p}=0.009)$; however, a similar reduction in pain in the therapeutic group of 1.7 points did not reach statistical significance $(\mathrm{p}=$ 0.072).

None $(0 \%)$ of the nerves transected prophylactically were complicated by postoperative neuralgia. Two $(3.8 \%)$ of the nerves transected therapeutically had recurrence of neuralgia, requiring further intervention.

CONCLUSIONS: Surgical neurectomies are performed for a variety of indications, both prophylactic and therapeutic, but their outcomes have been largely undefined. Among our patients, we show a nearly $4 \%$ risk of recurrent neuralgia if the neurectomy was performed therapeutically, i.e., for preoperative neuralgia. Interestingly, we did not show the same risk when neurectomy was performed prophylactically, such as a consequence of another operation, without pre-operative neuralgia.

The decision to perform a neurectomy should therefore be judicious and selective, as we show outcomes can be unpredictable particularly when performed therapeutically. We recommend that patients who undergo neurectomy, especially if indicated for neuralgia, be informed of the risk of postoperative neuralgia and need for further intervention.

\section{S039}

\section{Delayed Primary Closure of the Skin and Subcutaneous Tissues Following Complex Abdominal Wall Reconstruction: A Propensity-Matched Study}

Sullivan A Ayuso, MD; Bola G Aladegbami, MD; Sharbel A Elhage, MD; Angela M Kao, MD; Tanu Prasad, MA; Kent W Kercher, MD; Paul D Colavita, MD; Vedra A Augenstein, MD; B T Heniford, MD; Carolinas Medical Center.

Introduction: Wound complications following abdominal wall reconstruction (AWR) in a contaminated setting occur with a frequency of $40-50 \%$. When wound complications do occur, they lead to a 3-fivefold increase in rate of recurrence. Thusly, high risk patients were often discharged with negative pressure wound therapy (NPWT) but took an average of 127 days to heal. The purpose of this study was to examine the effect of short-term NPWT, followed by operative delayed primary closure (DPC) of the skin and subcutaneous tissue after AWR in a contaminated setting.

Methods: A prospective institutional hernia database was queried for patients who underwent DPC after AWR between 2010 - 2020. A non-DPC group was created using propensity-matching, accounting for age, BMI, defect size, CDC wound class, diabetes, smoking, and steroid use. Standard statistical methods were used, and a univariate analysis was performed between the DPC and non-DPC groups.

Results: In total, 124 patients underwent DPC following contaminated AWR. Patients had mean age $59.0 \pm 11.9$ years, BMI $32.1 \pm 6.7 \mathrm{~kg} / \mathrm{m} 2,28.2 \%$ diabetic, and $24.2 \%$ history of smoking. The hernias were on average very large $(216 \pm$ $165.8 \mathrm{~cm} 2)$ and often recurrent $(81.4 \%)$. All patients had CDC Class $3(16.3 \%)$ or 4 (84.7\%) wounds. Using the Carolinas Equation for Determining Associated Risks (CeDAR) score, these patients were predicted to have a $66.0 \%$ chance of developing a wound complication. Biologic mesh was used for all cases. Component separation was performed in $60.5 \%$ of cases and panniculectomy in $4.8 \%$. Mean time to DPC was $5.3 \pm 1.6$ days. Postoperatively, $29.8 \%$ patients developed a wound complication, and $13(10.5 \%)$ patients had a failed DPC requiring reopening of the wound The rate of recurrence was $2.1 \%$ with a mean follow-up time of $19.9 \pm 25.8$ months. After propensity-matching, there were 88 patients each in the DPC and non-DPC groups. DPC patients had fewer overall wound complications $(33.0 \%$ vs $51.1 \%, \mathrm{p}=$ 0.02 ). While $10.5 \%$ of the DPC group required NPWT, $21.8 \%$ of patients in the nonDPC had their wound opened and required long-term NPWT. Length of stay was one day shorter in the DPC group $(9.2 \pm 4.7$ vs $10.1 \pm 10.2, \mathrm{p}=0.04)$. Hernia recurrence was low overall $(1.1 \%$ vs $3.4 \%, p=0.49)$ with mean follow-up time of $21.3 \pm$ 24.5 months.

Conclusions: DPC can be performed with a high rate of success in complex AWR patients and improves rates of wound complications. In patients undergoing AWR in a contaminated setting, DPC should be strongly considered. 
S040

Polypharmacy predicts postoperative complications in older adults undergoing transversus abdominis release (TAR)

Julia L Hamilton; Bradley Kushner; Jeffrey Blatnik; Timothy Holden; Sara E Holden; Washington University School of Medicine.

Introduction: Although Transversus Abdominis Release (TAR) is an effective procedure for the repair of complex ventral hernias, it should not be considered low risk. This is particularly true for older adults, who are disproportionately affected by unique age-related risk factors. Among these risk factors is polypharmacy, defined as the concurrent use of multiple medications. While polypharmacy has been studied in other surgical fields and may predict worse outcomes, the effect of polypharmacy on older patients undergoing TAR is unclear.

Methods: This was a retrospective cohort study of patients $\geq 60$ years of age who underwent either open or robotic TAR by two abdominal wall specialists between $1 / 2018$ and 6/2020. Patients who underwent combined procedures or biologic meshbased repairs were excluded. Patients were categorized into tertiles based on the number of preoperative medications. Topical medications, vitamins, minerals and herbal supplements were excluded from medication counts. Outcomes included total number of postoperative complications (pneumonia, urinary tract infection, altered mental status, major adverse cardiovascular events, ileus, acute kidney injury, stroke, and venous-thromboembolism events), surgical site occurrence (SSO), length of hospital stay (LOS), percentage requiring ICU stays, and 30-day readmissions. ANOVA and Chi-Square tests were used for continuous and categorical variables, respectively.

Results: There were 132 patients eligible for inclusion. Patients in the first, second, and third tertiles were on $4.68 \pm 2.04,10.3 \pm 1.69$, and $17.9 \pm 3.64$ medications, respectively (mean \pm standard deviation). The mean age of the sample was 67.9 years, $38 \%$ were female, the mean BMI was $31.3 \mathrm{~kg} / \mathrm{m} 2$, and $80(60 \%)$ underwent open surgery, none of which differed significantly by tertile group. The third tertile had a significantly greater number of average complications per patient $(0.66)$ compared to the first $(0.25)$ and second tertiles $(0.25)(\mathrm{p}=0.03)$. There was a trend towards a greater proportion of patients in the third tertile requiring ICU stays $(15.9 \%)$, as compared to the first $(2.7 \%)$ and second tertiles $(6.8 \%)(\mathrm{p}=0.08)$. Similarly, the third tertile trended towards longer LOS (5.4), as compared to the first (3.2) and second tertiles (3.8) $(\mathrm{p}=0.06)$. However, there were no differences in the number of SSOs $(p=0.77)$ or readmissions $(p=0.97)$ between groups.

Conclusion: Polypharmacy is associated with an increased risk of complications following TAR in older adults. Moreover, polypharmacy may be associated with longer LOS and higher likelihood of ICU stay. A higher-powered study to better clarify these relationships is warranted. Addressing polypharmacy in older adults may optimize patient outcomes following a TAR

\section{S041}

\section{DISPARITIES IN UTILIZATION OF ROBOTIC SURGERY FOR COLON CANCER: AN EVALUATION OF THE U.S. NATIONAL CANCER DATABASE}

Michael L Horsey, $\mathrm{MD}^{1}$; Andrew Sparks, $\mathrm{MS}^{2}$; Matthew $\mathrm{Ng}, \mathrm{MD}^{3}$; Vincent Obias, $\mathrm{MD}^{5} ;{ }^{1}$ Walter Reed National Military Medical Center; ${ }^{2}$ George Washington University Medical Faculty Associates; ${ }^{3}$ George Washington University Department of Colon and Rectal Surgery.

Background: Despite demonstrated benefits of a minimally-invasive approach for colorectal procedures, significant disparities in access to these techniques still exist for the U.S. population. While these inequalities have been well-documented for laparoscopic techniques, few studies have evaluated discrepancies in use of the robotic platform. The objective of our study was to analyze whether disparities exist in the use of robotic surgery in treatment of colon cancer.

Methods: The U.S. National Cancer Database was queried for patients with nonmetastatic colon adenocarcinoma who underwent robot-assisted curative resection from 2010 to 2016. Demographic, clinicopathologic, and treatment facility-related variables were analyzed with respect to preferential utilization of robotic approach by way of multivariable logistic regression. Patients with metastatic disease, missing or incomplete demographic or surgical information, and those who went local tumor excision were excluded.

Results: After exclusions, 74, 984 cases were identified, 3001 (4\%) of which underwent a robotic approach. 245 patients $(8.5 \%)$ underwent conversion to open. Compared with younger, white, or privately insured patients, patients who were older (per 1 year increase, adjusted odds ratio $[\mathrm{aOR}]=0.99 ; \mathrm{p}<0.001)$, Black $(\mathrm{aOR}$ $=0.87 ; \mathrm{p}=0.023)$, or who were uninsured $(\mathrm{aOR}=0.48 ; \mathrm{p}<0.001)$, had Medicaid $(\mathrm{aOR}=0.67 ; \mathrm{p}<0.001)$, Medicare $(\mathrm{aOR}=0.89 ; \mathrm{p}=0.019)$, or unknown insurance status $(\mathrm{aOR}=0.67 ; \mathrm{p}=0.021)$ were significantly associated with decreased independent odds of undergoing a robotic approach. Additionally compared with patients with stage I disease or those who underwent segmental colectomy, patients who were diagnosed with stage II $(\mathrm{aOR}=0.73 ; \mathrm{p}<0.001)$, or stage III $(\mathrm{aOR}=0.69 ; \mathrm{p}<0.001)$ disease or underwent hemicolectomy $(\mathrm{aOR}=0.80 \mathrm{p}<0.001)$ or total abdominal colectomy $(\mathrm{aOR}=0.71 ; \mathrm{p}<0.001)$ were significantly associated with decreased independent adjusted odds of undergoing robotic surgery. Urban centers also had decreased adjusted odds of offering a robotic approach compared with metropolitan centers $(\mathrm{aOR}=0.80 ; \mathrm{p}<0.001)$. Finally, patients with higher education level $(\mathrm{aOR}=$ $1.27 ; \mathrm{p}<0.001)$, more recent year of surgery $(2016 \mathrm{v} 2010 ; \mathrm{aOR}=8.32 ; \mathrm{p}<0.001)$, and higher procedure volume centers $(\mathrm{aOR}=1.18 ; \mathrm{p}=0.014)$ were significantly associated with increased independent adjusted odds of receipt of a robotic approach compared with those with lower education levels, earlier years of surgery, or lower volume centers.

Conclusion: The robotic approach is increasing in popularity for treatment of nonmetastatic colon cancer. Despite this, significant inequalities in utilization of the robotic platform exist with respect to patient demographics and socioeconomic factors, and access may be limited to certain facility types. As advantages of the robotic platform become better understood, these disparities should be addressed to provide for equal access to all patients. 


\section{S042}

\section{Urban-Rural Disparities in Utilization of Minimally Invasive Surgery for Colon Cancer.}

Andrew Harner ${ }^{1}$; Bao-Ling Adam, $\mathrm{PhD}^{1}$; Frank D Lewis, $\mathrm{PhD}^{2}$; Jaine McKenzie, $\mathrm{MD}^{1}$; Jim Kruse, $\mathrm{DO}^{1} ;{ }^{1}$ Medical College of Georgia at Augusta University; ${ }^{2} \mathrm{Neu}-$ roRestorative Research Institute, Medical College of Georgia at Augusta University.

Introduction: Surgical treatment for colon cancer has rapidly evolved in the past decade, with a trend towards minimally invasive surgery (MIS), including both laparoscopic and robotic approaches. The use of MIS over traditional laparotomy for colon cancer results in shorter lengths of stay and fewer complications. Despite the obvious benefits of utilizing MIS for appropriately selected patients, there exists an apparent disparity in its utilization based on factors such as type of insurance and hospital location. Our study aims to observe the trends in MIS usage for colon cancer based on patient location (rural versus urban). We hypothesized that the utilization rates for MIS in colon cancer in more rural areas would lag behind those living in a more urban setting, and that complications would also be higher in the rural setting. Methods: The National Inpatient Sample (NIS) database (years 2008-2016) was queried to identify patients with a diagnosis of colon cancer who underwent elective colectomy. These patients were stratified based on their location as either urban (county population density of $>250,000$ ) or rural (county population of $<250,000$ ). Patients were further stratified based on the procedure performed (either open, laparoscopic, or robotic). Statistical analysis was performed to compare length of stay, complication rates, and mortality rates between the different procedure types and the urban and rural patient populations.

Results: Utilization of laparoscopic and robotic surgery was significantly higher for patients living in urban areas ( 40.5 and $4.6 \%$, respectively) compared to rural areas ( 29.8 and $2.7 \%$, respectively). Open procedures were associated with a significantly longer average length of stay ( 8.2 days) compared to laparoscopic (5.4 days) and robotic procedures (5.0 days). Laparoscopic and robotic procedures had significantly fewer complications ( 8.3 and $8.2 \%$, respectively) compared to open procedures $(16.9 \%)$. Patients from rural areas experienced fewer complications from open procedures than their urban counterparts (14.6 versus $18.2 \%$ ) but suffered higher complication rates from laparoscopic procedures (10.4 versus $7.7 \%$ ). Patients from rural locations had a significantly higher mortality rate $(1.5 \%)$ compared to patients from urban locations $(1.0 \%)$, though there was no significant different in mortality between open, laparoscopic, or robotic procedures.

Discussion: MIS for elective colectomies in colon cancer patients results in significantly shorter lengths of stay and fewer complications. However, patients from rural areas are undergoing fewer MIS procedures than their urban counterparts. Appropriate referral to centers accustomed to performing MIS for colon cancer may decrease this disparity and improve overall outcomes.

\section{S043}

\section{Predictors of operative difficulty in robotic low anterior resection for rectal cancer}

Jonathan B Yuval, MD; Hannah M Thompson, MD; Megan Fiasconaro, MS; Sujata Patil, PhD; Iris H Wei, MD; Emmanouil P Pappou, MD, PhD; J. Joshua Smith, MD $\mathrm{PhD}$; Jose G Guillem, MD, MPH; Garrett M Nash, MD, MPH; Martin R Weiser, MD; Philip B Paty, MD; Julio Garcia-Aguilar, MD, PhD; Maria Widmar, MD, MPH; Memorial Sloan Kettering Cancer Center.

Background: Previous studies investigating predictors of operative difficulty in robot-assisted low anterior resection of rectal cancer were of limited sample size, heterogenous surgical approach, and/or based on data from a single surgeon. This study evaluates the effect of pre-operative clinical and anatomical parameters on operative difficulty in robotic low anterior resection performed by experienced surgeons in a high-volume colorectal cancer practice.

Methods: Patients who underwent robotic low anterior resection by four expert surgeons between January 2016 and June 2019 were included. Baseline clinical data and preoperative pelvimetry measurements were collected. Increasing operative time was used as a measure of operative difficulty. Univariate and multivariate mixed models were used to identify significant predictors of increasing operative time, with surgeon as a random effect, thereby controlling for variability in surgeon speed and proficiency. A composite outcome of operative difficulty including conversion, positive margins and incomplete mesorectum was investigated in a secondary analysis.

Results: This study enrolled 382 patients. Median (minimum-maximum) operative time was 4.28 (1.95-11.33) hours but varied by individual surgeon (3.45 (1.95-6.10), 3.83 (2.45-8.55), $5.40(2.93-9.60)$ and 5.93 (3.33-11.33) hours, $\mathrm{p}<0.001)$. Predictors of longer operative time in univariate analysis included male sex, body mass index, neoadjuvant radiotherapy, low tumor height, anterior tumor location, sacral height, pubic tubercle height and mesorectal area at the S5 vertebral level. All but anterior tumor location and pubic tubercle height remained significant on multivariate analysis. Conversion was performed in 2 cases $(0.5 \%)$, whereas incomplete mesorectum and positive margins were found in $30(7.9 \%)$ and $19(5.0 \%)$ patients, respectively. Only pelvic outlet length was associated with this composite outcome. Conclusions: Herein we describe pre-operative clinical and anatomic predictors of operative difficulty in robotic low anterior resection for surgeons with different operative speed. Evaluating these parameters before surgery can help in surgical preparation, selection of appropriate teaching cases, hospital resource planning and management of patient expectations. 
S045

\section{The Success Rate of Robotic Natural Orifice Intracorporeal Anastomosis and Transrectal Extraction (NICE) in a Large Cohort of Consecutive Unselected Patients.}

Thais Reif de Paula, MD ${ }^{1}$; Roberto Luna-Saracho, $\mathrm{MD}^{1}$; Jean-Paul LeFave, MD ${ }^{2}$; Melissa S Smith, $\mathrm{DO}^{2}$; Eric M Haas, $\mathrm{MD}^{1}$; ${ }^{1}$ College of Medicine-University of Houston; ${ }^{2}$ Houston Colon.

Background: The Robotic NICE procedure is a total intracorporeal approach in which specimen extraction and anastomosis is accomplished without an abdominal wall incision other than the port sites themselves. Despite numerous benefits including the elimination of surgical site and hernia risk, it is estimated that fewer than $2 \%$ of left-sided resections are accomplished in this fashion. We aim to present the success rate of the NICE procedure in a large cohort of unselected consecutive patients presenting with benign and malignant disease.

Methods: All patients who presented for elective left-sided colorectal resection (anterior or low anterior resection) for benign or malignant disease between May 2018 and June 2020 underwent the robotic NICE procedure and were prospectively entered in an IRB database. Data abstracted included demographic, clinical data, disease features, intervention data, and outcomes data. Surgical outcome included success rate of Intracorporeal anastomosis (ICA) and transrectal extraction (TE) of specimen, conversion rate, and presence of anal sphincter injury.

Results: A total of 211 cases were performed (53\% female) with the mean age of 57.9 years and mean BMI of $28.8 \mathrm{~kg} / \mathrm{m} 2$. The mean operative time was $227.5 \mathrm{~min}$ (range 107-547). There was one conversion. The mean length of stay was 2.62 days (range 1-15). The postoperative complication rate was $10.4 \%(\mathrm{n}=22)$ including postoperative ileus $1.4 \%(\mathrm{n}=3)$ and organ space SSI $5.2 \%(\mathrm{~N}=11)$. The readmission rate was $7.1 \%(\mathrm{n}=15)$, and the reoperation rate was $4.7 \%(\mathrm{n}=10)$. In all cases, an intracorporeal anastomosis was successfully accomplished. Overall, transrectal extraction was achieved in $94.3 \%(\mathrm{n}=199)$ of cases. When evaluated by disease, $100 \%$ of those with benign disease successfully had TRE however $44 \%$ required thinning of the mesentery. For malignant cases, $21 \%$ could not be removed via TRE due to tumor size greater than $3 \mathrm{~cm}$. shaving of the mesentery was not allowed in malignant cases. One patient in the malignant group had a sphincter injury which resolved.

Conclusion: The NICE procedure is associated with a very high success rate and a very low postoperative complications rate. In all benign cases, transrectal extraction of specimen can be achieved. In cases where there is a bulky mesentery or lesions larger than $3 \mathrm{~cm}$ tend to require an abdominal wall extraction incision.

\begin{tabular}{|l|c|c|}
\hline Figure 1. Success rates and factors for ICA and TRE ( $\mathrm{n}=211)$ \\
\hline & \multicolumn{2}{|c|}{ Diagnosis } \\
\hline & Benign $(\mathrm{n}=152)$ & Malignant $(\mathrm{n}=59)$ \\
\hline Intracorporeal anastomosis (ICA) & $152(100 \%)$ & $59(100 \%)$ \\
\hline Transrectal specimen extraction & $152(100 \%)$ & $47(79.7 \%)^{*}$ \\
\hline Thinning of mesentery required & $29(44 \%)$ & $\mathrm{n} / \mathrm{a}$ \\
\hline Conversions & $1(0 \%)$ & $0(0 \%)$ \\
\hline Sphincter injury & $0(0 \%)$ & $1(\%)$ \\
\hline $\begin{array}{l}* \\
\text { * all tumor size }<3 \mathrm{~cm} / \mathrm{A}-\text { mesentery thinning contra indicated in malignant cases }\end{array}$ \\
\hline
\end{tabular}

\section{S046}

\section{Outcomes in patients with locally advanced colon cancer} undergoing operative conversion

Keegan Guidolin, MD ${ }^{1}$; Deanna Ng, MBBS ${ }^{1}$; Sami Chadi, MD, $\mathrm{MSc}^{2}$; Fayez Quereshy, MD, MBA'; ${ }^{1}$ University of Toronto; ${ }^{2}$ University Health Network.

Introduction: Surgeons may choose an open approach to locally advanced colon cancer (LACC) because of the elevated rate of conversion (from minimally invasive to open) in these patients. Poorer outcomes have been suggested in those undergoing converted procedures but have not yet been studied in a locally advanced population. We sought to compare perioperative outcomes in patients with $\mathrm{T} 4 \mathrm{~N} 2$ colon cancer undergoing minimally invasive surgery (MIS), planned open (PO), and converted $(\mathrm{CN})$ procedures to evaluate the influence of conversion in this subgroup.

Methods: A retrospective cohort study was conducted using the NSQIP database. Patients with T4N2 colon cancer undergoing elective resection were included; rectal/ unknown tumour location, and T4b disease were excluded. Patients were divided into cohorts based on approach: PO, MIS, and CN. Summary statistics were compared between groups. Multivariable analysis was conducted for mortality and morbidity outcomes.

Results: 1,286 cases were included (313 PO, 842 MIS, $131 \mathrm{CN}$ ); $10.2 \%$ underwent conversion. Those undergoing MIS had a shorter median length of stay than those undergoing $\mathrm{PO}$ or $\mathrm{CN}$ (4 days MIS vs 6 days $\mathrm{PO}$ and $\mathrm{CN}, \mathrm{p}<0.0001$ ). Those undergoing PO were least likely to receive a primary anastomosis ( $88 \%$ PO vs $91 \%$ $\mathrm{CN}$ vs $95 \%$ MIS, $\mathrm{p}=0.0004)$ and $\mathrm{CN}$ were least likely to receive a stoma (37\% vs $42 \%$ MIS vs $51 \% \mathrm{PO}, \mathrm{p}=0.0082$ ). On univariable analysis $\mathrm{CN}$ resulted in increased rates of any complication ( $34 \%$ vs $15 \%$ MIS vs $28 \%$ PO, p < 0.0001$)$, including superficial and deep infection $(\mathrm{p}=0.0002, \mathrm{p}=0.0137)$, pneumonia $(\mathrm{p}=0.0039)$, and prolonged ventilator dependence $(\mathrm{p}<0.0001)$. $\mathrm{CN}$ also had a greater rate of anastomotic leak ( $8 \%$ vs $3 \%$ MIS vs $4 \%$ PO, p $=0.0046)$ and death $(4 \%$ vs $1 \%$ MIS vs $2.6 \% \mathrm{PO}, \mathrm{p}=0.05)$. On multivariable analysis, significant predictors of any complication included age, ASA class, $\mathrm{M}$ stage, and approach; however, $\mathrm{CN}$ did not increase the risk of complication compared with MIS, whereas PO nearly doubled the risk of complication $(\mathrm{OR}=1.98, \mathrm{p}=0.0083)$. The only significant predictor of mortality on multivariate analysis was age $(\mathrm{HR}=1.09, \mathrm{p}=0.0002)-$ approach was not associated with mortality.

Conclusion: PO confers the greatest risk of suffering any complication, and patients in this category are least likely to receive a primary anastomosis, whereas patients undergoing CN are least likely to receive stoma. Surgical approach was not associated with death. Results of our study challenge the notion that conversion is associated with the worst perioperative outcomes and an MIS approach should be considered in patients with LACC. 
S047

Tolerating clear fluids diet on postoperative day 0 predicts early recovery of gastrointestinal function and better outcomes after laparoscopic colectomy

V Leung; N Alsharqawi; G Baldini; AS Liberman; P Charlebois; B Stein; LS Feldman; Jr JF Fiore; L Lee; McGill University.

Introduction: A high proportion of colorectal surgery patients within an enhanced recovery pathway(ERP) do not experience complications but remain hospitalized mainly waiting for gastrointestinal(GI) recovery. Accurate identification of these patients may allow discharge prior to the return of GI function. We hypothesize that earlier toleration of oral intake predicts quick GI recovery. Therefore, the objective of this study is to determine if clear fluid(CF) toleration on postoperative day(POD) 0 was associated with uncomplicated return of GI function after laparoscopic colorectal surgery.

Methods: Pooled data from three prospective studies from a single specialist colorectal referral centre were analyzed(2013-2019). All patients were managed by an ERP with a 3-day target length of stay(LOS). The present study only included adult patients that underwent elective laparoscopic colectomy without stoma. Postoperative GI symptoms were collected daily in all three datasets. The main exposure variable whether $\mathrm{CF}$ diet was tolerated on POD0, defined as patients drinking at least $300 \mathrm{~mL}$ of $\mathrm{CF}$ without any nausea, anti-emetics, or vomiting (CF + vs $\mathrm{CF}-)$. $\mathrm{CF}$ - also included patients that were not given $\mathrm{CF}$ on POD0 The main outcome measure was time to GI-3(tolerating solid diet and passage of gas or stools). A subset of patients completed daily I-FEED, a validated assessment of GI function $(6+$ points $=$ severe GI dysfunction).

Results: A total of 221 patients were included in this study, including $69 \% \mathrm{CF}+$ and $31 \% \mathrm{CF}-$. The groups were similar in age, gender, and comorbidities, but the $\mathrm{CF}-$ patients were more likely to have surgery for inflammatory bowel disease. CF + patients had faster time to GI-3(mean 1.6d(SD0.7) vs. 2.3d(SD1.5),p < 0.001), including both faster time to toleration of solid diet and flatus. CF- patients were more likely to require a nasogastric tube during the first $72 \mathrm{~h}$ after surgery $(19 \% \mathrm{vs}$. $3 \%, \mathrm{p}<0.001)$. The $\mathrm{CF}+$ group also experienced fewer complications $(19 \%$ vs. $35 \%, \mathrm{p}=0.009)$, shorter mean LOS (mean 3.6d(SD2.9) vs. $6.2 \mathrm{~d}(\mathrm{SD} 9.4), \mathrm{p}=0.002)$, and were more likely to be discharged by the target $\operatorname{LOS}(66 \%$ vs. $50 \%, \mathrm{p}=0.024)$. There were 147 patients that completed I-FEED scores $(C F+n=102, C F-n=45)$. Patients in the CF + group had lower I-FEED scores over the first $72 \mathrm{~h}$ (Figure) and were less likely to experience postoperative GI dysfunction( $6 \%$ vs. $29 \%$,p < $<.001$ ). Conclusion: Toleration of $\mathrm{CF}$ on POD0 was associated with faster return of GI function, fewer complications, and shorter LOS. This may be used as a criteria for potential discharge prior to full return of GI function after laparoscopic colectomy within an enhanced recovery pathway.

Figure 1 - Daily I-FEED scores in patients who tolerated clear fluid diet on PODO $(n=102)$ versus those that did not $(n=45)$

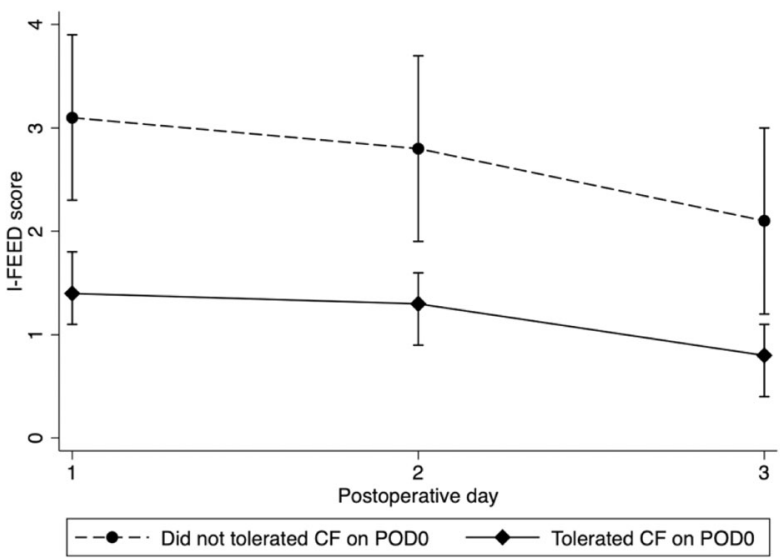

\section{S048}

\section{Predicting Risk of Venous Thromboembolism in Patients Undergoing Diverticular Resection}

Constantine M Poulos, $\mathrm{MD}^{1}$; Rachel Scott, $\mathrm{DO}^{2}$; Dorothy Wakefield, PStat ${ }^{1}$; Robert Lewis, MD ${ }^{2}$ ' ${ }^{1}$ University of Connecticut; ${ }^{2}$ Colon and Rectal Surgeons of Greater Hartford.

Introduction: Following colorectal surgery, venous thromboembolism (VTE) is a serious complication occurring at an estimated incidence of $2-4 \%$. There is a significant body of literature stratifying risk of VTE in specific populations undergoing colorectal resection for cancer or inflammatory bowel disease. There has been little research characterizing patients undergoing colorectal surgery for other indications, e.g. diverticulitis. We hypothesize that there exists a subgroup of patients with identifiable risk factors undergoing resection for diverticulitis that has relatively higher risk for VTE. Treatment strategies regarding diverticulitis has evolved within the last several decades with an increased utilization of non-operative or interventional management techniques which defer definitive operative interventions. These alterations in treatment strategies may modify the risk profile of diverticulitis patients over time. In this analysis, we provide the largest, up-to-date characterization of VTE in patients undergoing colorectal resection for diverticulitis.

Methods: We conducted a retrospective review of the American College of Surgeons National Surgical Quality Improvement Project database from 2006 - 2017 who underwent colorectal resection for diverticulitis. Patients with a primary indication for resection other than diverticulitis were excluded. Multivariate logistic regression modeling was conducted to determine the risk of VTE for each independent variable. A novel scoring system was developed and a receiver-operatingcharacteristic curve was generated.

Results: The rate of DVT/PE was $1.2 \%$ and $0.62 \%$, respectively. There was no significant difference in the rate of VTE between years. Significant risk factors for VTE included age, length of stay, race, presence of bleeding disorder, baseline functional status, perioperative transfusion, perioperative steroids, and perioperative sepsis. An 8-point scoring system was developed using identified significant variables. The area under the receiver-operating-characteristic curve was 0.78 (Fig. 1) Patients scoring $\geq 2$ on the developed scoring scale had an increased risk of postoperative VTE (OR $=5.72,95 \%$ CI $4.49-7.3$ ).

Conclusion: Some patients undergoing colonic resection for diverticulitis are at significantly higher risk for perioperative VTEs. A simple 8-point scoring system based on identified significant risk factors was specifically developed to predict the risk of VTE in patients undergoing diverticular colorectal resection.

Figure 1

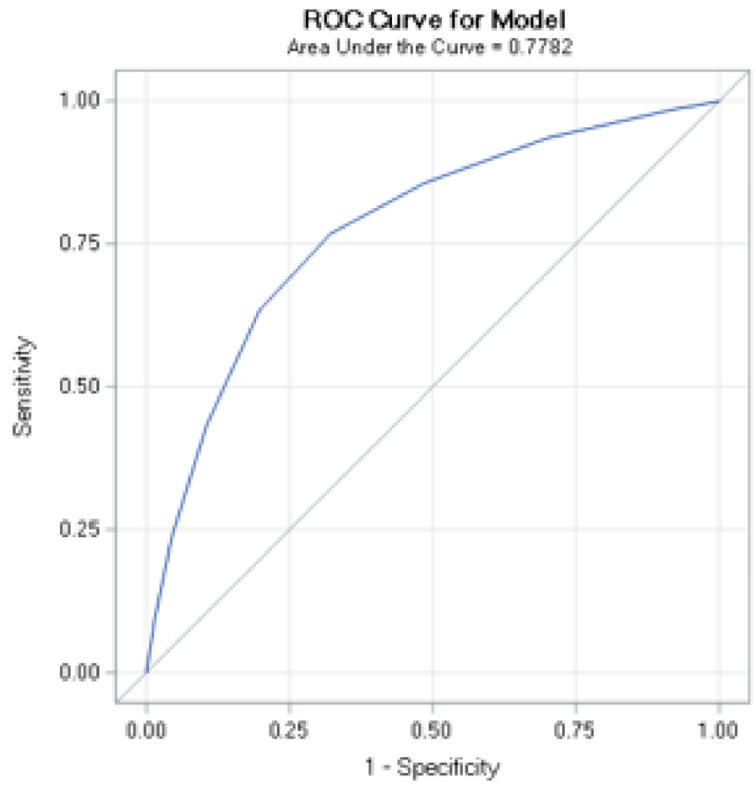


S24

Surgical Endoscopy (2021) 35:S1-S103

S049

Impact of obesity on postoperative outcomes in transanal total mesorectal excision for rectal cancer

Daniel K Peyser, $\mathrm{MD}^{1}$; Makda Zewde ${ }^{1}$; Antoinette Bonaccorso, $\mathrm{MD}^{1}$; Tarim Alavi, $\mathrm{MD}^{2}$; Robert Goldstone, $\mathrm{MD}^{3}$; John H Marks, $\mathrm{MD}^{4}$; Justin A Maykel, MD ${ }^{2}$; Elisbeth C McLemore, MD ${ }^{5}$; Dana Sands, MD ${ }^{6}$; Scott R Steele, MD ${ }^{7}$; Steven D Wexner, $\mathrm{MD}^{6}$; Mark Whiteford, $\mathrm{MD}^{8}$; Patricia Sylla, MD ${ }^{1} ;{ }^{1}$ Icahn School of Medicine at Mount Sinai; ${ }^{2}$ University of Massachusetts Medical School; ${ }^{3}$ Massachusetts General Hospital; ${ }^{4}$ Lankenau Institute for Medical Research; ${ }^{5}$ Kaiser Permanente Los Angeles Medical Center; ${ }^{6}$ Cleveland Clinic Florida; ${ }^{7}$ Cleveland Clinic Lerner College of Medicine of Case Western Reserve University; ${ }^{8}$ Providence Portland Medical Center.

Background: Over the last decade, taME has been increasingly adopted in the curative resection of low rectal cancer. When combined with an abdominal approach, taTME facilitates distal rectal dissection. Obesity is a known risk factor for conversion during laparoscopic and robotic TME and is associated with increased morbidity and suboptimal TME specimen quality. This multicenter study aimed to compare short-term postoperative and pathologic outcomes of taME between nonobese versus obese patients across high-volume rectal cancer centers in the United States.

Methods: A retrospective analysis of all consecutive taME cases performed for primary rectal cancer at 8 tertiary centers was conducted. Perioperative and postoperative outcomes were compared between non-obese versus obese patients (BMI $<30 \mathrm{vs} \geq 30 \mathrm{~kg} / \mathrm{m} 2$ ). Univariate analysis was performed using Student's t-test/ Mann-Whitney-U test for continuous variables and Chi-squared/Fisher-exact test for categorical variables.

Results: Between November 2011 and June 2020, 396 taME procedures were performed in $276(69.9 \%)$ non-obese and $120(30.1 \%)$ obese patients with a median BMI of $27.4 \mathrm{~kg} / \mathrm{m} 2$ (IQR 24.1-30.8). Males comprised $68.4 \%$ vs $66.6 \%$ of each cohort, respectively, with no significant differences in tumor stage or neoadjuvant treatment across the groups (Table 1). Median tumor height from the anal verge (AV) was 5.3 vs $6.0 \mathrm{~cm}$ in the non-obese vs obese group $(\mathrm{p}=0.037)$ with a $96.7 \%$ vs. $93.3 \%(p=0.124)$ rate of sphincter preservation, respectively. Operative time was shorter in the non-obese group ( 345 vs $384 \mathrm{~min}, \mathrm{p}=0.002)$, with no significant differences in conversion rates $(2.5 \%$ vs $6.7 \%, \mathrm{p}=0.08)$, blood loss ( $100 \mathrm{vs} 150 \mathrm{ml}, \mathrm{p}$ $=0.113)$, intraoperative complications $(5.1 \%$ vs $7.5 \%, \mathrm{p}=0.343)$ or median hospital stay ( 5 days, $\mathrm{p}=0.325)$. No significant differences in postoperative complications $(37.3 \%$ vs $47.5 \%, \mathrm{p}=0.058)$ were noted between non-obese and obese cohorts, including Clavien-Dindo grade $\geq 3$ complications $(13.6 \%$ vs $24.6 \%, \mathrm{p}=0.08)$, anastomotic complications $(5.4 \%$ vs $10 \%, \mathrm{p}=0.098)$, or reoperation rates $(1.8 \%$ vs $0 \%, \mathrm{p}=0.328)$. The rate of complete/near complete TME specimen $(94.5 \% \mathrm{vs} 95 \%, \mathrm{p}$ $=0.849)$, positive circumferential radial $(2.9 \%$ vs $0.8 \%, \mathrm{p}=0.288)$ or distal margins $(0.4 \%$ vs $0 \%, \mathrm{p}>0.999)$, and median number of lymph nodes (18 vs $17, \mathrm{p}=0.155)$ did not differ significantly between the groups.

Conclusion: In this large multicenter study, taTME was predominately preformed in males for tumors located $\leq 6 \mathrm{~cm}$ from the AV. Despite a BMI $\geq 30$ in $1 / 3$ of the cohort, sphincter preservation was achieved in $95.7 \%$ with low conversion and complication rates with similar short-term oncologic outcomes. TaME is a valuable tool for the management of distal rectal tumor, especially in obese males.
Table 1: Perioperative and pathologic outcomes

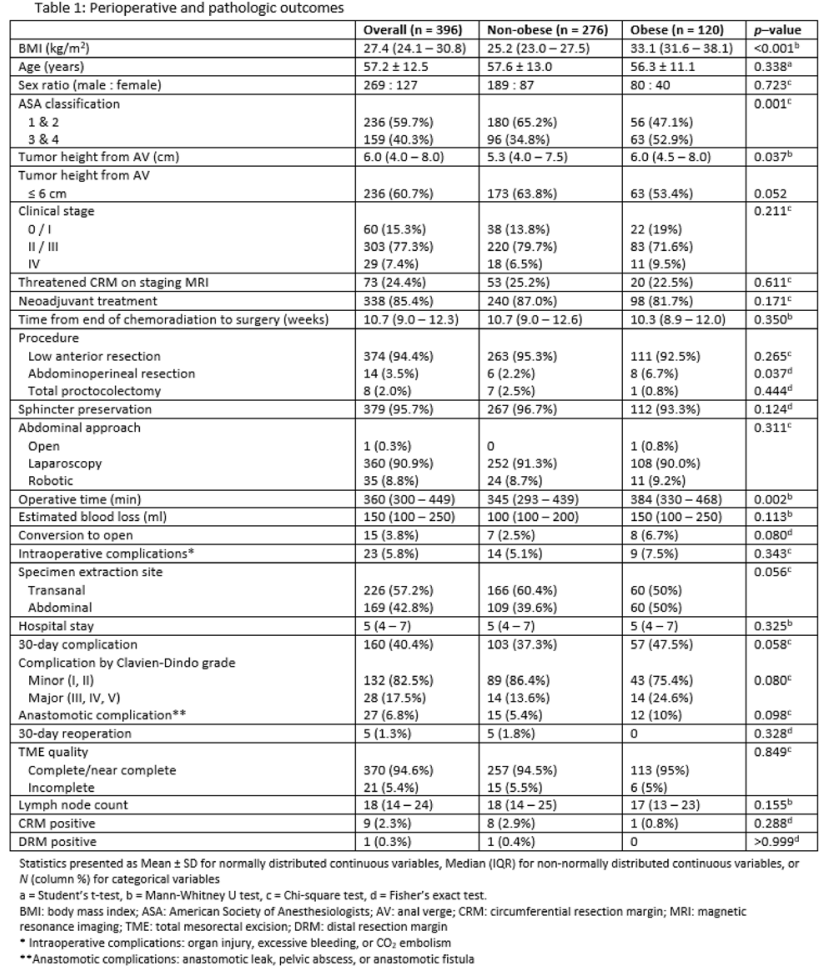

Springer 
S050

\section{OPEN VERSUS MINIMALLY INVASIVE SMALL BOWEL RESECTION FOR CROHN'S DISEASE: A NSQIP RETROSPECTIVE REVIEW AND ANALYSIS}

Michael L Horsey ${ }^{1}$; Andrew Sparks, $\mathrm{MS}^{2}$; Matthew Ng, $\mathrm{MD}^{3}$; Vincent Obias, $\mathrm{MD}^{3} ;{ }^{1}$ Walter Reed National Military Medical Center; ${ }^{2}$ George Washington University Medical Faculty Associates; ${ }^{3}$ George Washington University Department of Colon and Rectal Surgery.

INTRODUCTION: Despite the preference for medical management of patients with Crohn's disease, many will require surgical resection at some point in their lifetime. Many studies have addressed surgical managment of ileocecal Crohn's disease, but few have evaluated outcomes of segmental resection for other portions of the small bowel. Therefore, the primary aim of this study was to compare outcomes for open and minimally-invasive surgical (MIS) approaches for patients with small bowel Crohn's disease excluding the ileocecal junction.

METHODS: The American College of Surgeons National Surgical Quality Improvement Program (NSQIP) was queried for patients diagnosed with Crohn's disease or complications related to Crohn's disease who underwent small bowel resection utilizing an open or MIS (to include laparoscopic and robot-assisted) approaches between 2012 and 2018. Patients with ascites, disseminated cancer, preoperative sepsis, ASA class 5, patients requiring mechanical ventilation, and those who underwent ileocecectomy were excluded. The association of preoperative variables including patient demographic information and comorbidities with surgical approach were examined using Fishers exact test. Intraoperative, and 30-day postoperative outcomes were compared between the groups using both univariate and multivariate logistical regression models. SAS was used for data analysis with $\mathrm{p}<$ 0.05 considered significant.

RESULTS: After exclusions, there were 1,697 patients with Crohn's disease who underwent segmental small bowel resection, 1252 of whom underwent open surgery and 445 of whom underwent MIS. The mean age of patients was $44.6 \pm 16.0$, and mean BMI was $25 \pm 6.47 \%$ of patients were female, $79 \%$ of patients were white, and $7 \%$ were black. Patients who received MIS were younger and more likely to have independent functional status and less likely to have pre-operative infection, low serum albumin, low hematocrit, or to undergo emergency surgery (respective $\mathrm{p}$ $<0.05)$. After adjusting for possible confounders with multivariable analysis, patients who underwent open surgery had a higher incidence of wound events (surgical site, organ space, or deep wound infection, or dehiscence) $(p=0.0004)$, post-operative bleeding $(\mathrm{p}=0.002)$, and return to operating room $(\mathrm{p}=0.046)$ compared with patients who underwent MIS. No significant differences were found between approaches with respect to major cardiac, renal, or pulmonary events, venous thromboembolism, post-operative sepsis, or 30-day mortality.

CONCLUSIONS: This retrospective review of NSQIP demonstrates that a minimally-invasive approach for segmental small bowel resection is associated with equivalent or improved morbidity over open surgery for select patients with Crohn's disease. Additional studies are needed to determine long-term outcomes of this approach.

\section{S051}

\section{IS THE HARTMANN'S PROCEDURE FOR DIVERTICULITIS OBSOLETE? NATIONAL TRENDS IN COLECTOMY FOR DIVERTICULITIS IN THE EMERGENCY SETTING FROM 1993-2015}

Hatim Alsulaim; Richard Garfinkle; Safiya Al-Masrouri; Nancy Morin; Carol-Ann Vasilevsky; Marylise Boutros; Jewish General Hospital.

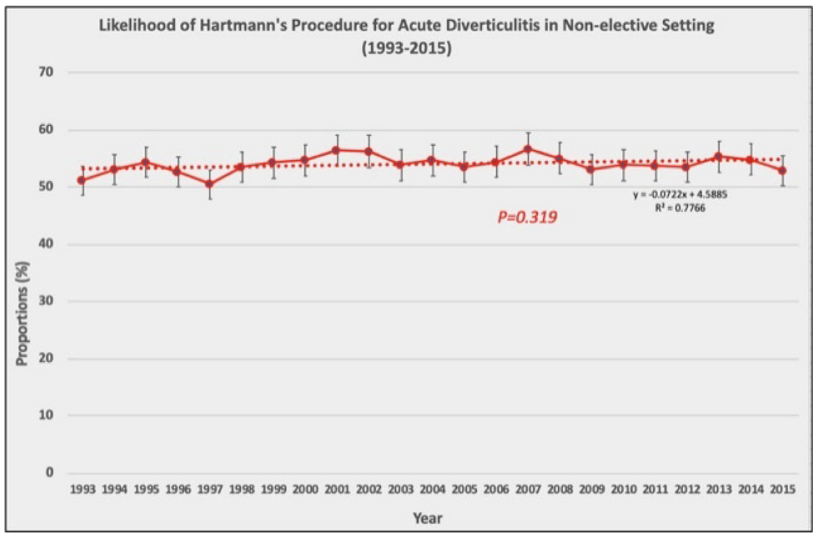

Background: Historically, Hartmann's procedure (HP) has been the operation of choice for diverticulitis in the emergency setting. However, recent evidence has demonstrated the safety of primary anastomosis (PA) with or without diverting ileostomy. The purpose of this study was to evaluate the trends of, and factors associated with, HP compared to PA in emergency surgery for diverticulitis over two decades.

Methods: Using the National Inpatient Sample database, we identified adult patients $\geq 18$ years- old who underwent emergency surgery for diverticulitis (HP or PA) between January 1993 and October 2015 using ICD-9 codes. Patients with inflammatory bowel disease, gastrointestinal cancer or who underwent elective diverticulitis surgery were excluded. Trends in HP were analyzed using multivariable linear regression, and factors associated with HP were assessed with multiple logistic regression

Results: Of 90,815 patients who underwent colectomy in the emergency setting for acute diverticulitis, $49,259(54.2 \%)$ had a HP and $41,556(46.8 \%)$ had a primary anastomosis (PA). Median age was 62 years (IQR: $50-73$ ), $52.8 \%$ were women, and $69.4 \%$ were of white race. Patients who had HP were more likely to be older (median 63 years $(51-75)$ vs. 60 years $(48-72), \mathrm{p}<0.001)$ ) and have major or extreme severity of illness index $(69.5 \%$ vs $41.4 \%, \mathrm{p}<0.001)$. The overall likelihood of HP did not differ significantly over time - HP comprised $53.5 \%$ of included cases in $1993-95$ and $54.3 \%$ of cases in $2013-15$ ( $p<0.001$ ). Of those who had a PA, 5.2\% had a concomitant diverting ileostomy; this increased over time, from $1.2 \%$ to $10.1 \%$ $(\mathrm{p}<0.001)$. On multiple logistic regression, age (ref: $18-44$ years; $45-54$ years: OR $1.13,95 \%$ CI $1.07-1.19 ; 55-64$ years: OR $1.21,95 \%$ CI $1.14-1.28 ; 65-74$ years: OR $1.27,95 \%$ CI $1.19-1.36 ; \geq 75$ years: OR $1.39,95 \%$ CI $1.29-1.49]$, male sex (OR $1.15,95 \% \mathrm{CI} 1.11-1.19)$, severity of illness [moderate illness: OR 1.81, 95\%CI 1.69-1.93; major/extreme illness: OR 4.80, 95\%CI 4.51-5.12], and weekend admission (OR 1.41, 95\% CI 1.35-1.46) were independent predictors of HP.

Conclusions: Despite early data demonstrating its safety compared to the Hartmann's Procedure, the use of sigmoid colectomy with primary anastomosis for diverticulitis in the emergency setting has remained stable over two decades. Future research should aim to identify a possible change in practice with the publication of more recent randomized controlled trials. 


\section{S052}

\section{TRENDS IN EARLY DISCHARGE AFTER COLORECTAL CANCER RESECTION AND PREDICTORS OF 30-DAY READMISSIONS: A NATIONWIDE READMISSIONS DATABASE ANALYSIS}

Jeongyoon Moon; Allison J. Pang; Gabriela Ghitulescu; Julio Faria; Carol-Ann Vasilevsky; Nancy Morin; Marylise Boutros; Jewish General Hospital.

Introduction: Implementation of enhanced recovery pathways in colorectal surgery has been effective in reducing length of stay, healthcare costs, and improving patient satisfaction. Readmissions following early discharge among colorectal cancer patients are believed to be low, however remain understudied. The aim of this study was three-fold: (i) to describe trends in early post-operative discharge and the associated hospitalization costs; (ii) to explore patient outcomes and resource utilization following early discharge; and (ii) to identify predictors of readmission following early discharge.

Methods: This was a retrospective cohort study using the Nationwide Readmissions Database. Adult patients admitted with a primary colorectal neoplasm who underwent colectomy or proctectomy between 2010-2017 were identified using ICD-9/10 codes. The exposure of interest was early post-operative discharge defined as $\leq$ 3 days from surgery. Main outcome measures were 30-day readmissions, post-operative complication rates, length of stay (LOS) and cost.

Results: In total, 342,242 patients were identified, and of those, 51,977 patients $(15.2 \%)$ had early discharges. The proportion of early discharges significantly increased ( $\mathrm{R} 2=0.95$ ), from $9.7 \%$ in 2010 to $23.4 \%$ in 2017 , while 30 -day readmission rates significantly declined $(\mathrm{R} 2=0.81)$ during the same time period; however, readmission rates among early discharges remained unchanged (mean $6.7 \% \pm 0.5$ ). A rise in the cost of index admissions and readmissions was observed among patients discharged early (Fig. 1). Compared to patients who had complications during the index admission, those who were discharged early and bounced back (readmitted within 7 days of discharge) with complications had higher rates of anastomotic leak $(38.4 \%$ vs $27.5 \%, p<0.001)$, bleeding $(5.3 \%$ vs $4.4 \%, p=0.007)$, and percutaneous drainage $(7.8 \%$ vs. $4.3 \%, \mathrm{p}<0.001)$. Complications that occurred following early discharge rather than during the index admission and required readmission were independent predictors of longer overall LOS $(b=0.044, p<$ $0.001)$ and higher hospitalization cost $(b=0.031, \mathrm{p}<0.001)$ after adjusting for clinically relevant factors. On multiple logistic regression, factors independently associated with 30-day readmission following early discharge were: male gender $(\mathrm{OR}=1.24,95 \% \mathrm{CI} 1.15-1.34)$; rectal cancer $(\mathrm{OR}=1.31,95 \% \mathrm{CI} 1.20-1.44)$; open surgery $(\mathrm{OR}=1.34,95 \% \mathrm{CI} 1.23-1.45)$; presence of stoma $(\mathrm{OR}=1.46,95 \% \mathrm{CI}$ 1.24-1.72); comorbidities including congestive heart failure $(\mathrm{OR}=1.38,95 \% \mathrm{CI}$ $1.13-1.68)$, chronic pulmonary disease $(\mathrm{OR}=1.29,95 \% \mathrm{CI} 1.14-1.45)$, renal failure $(\mathrm{OR}=1.32,95 \% \mathrm{CI} 1.12-1.55)$, and mood disorder $(\mathrm{OR}=1.20,95 \% \mathrm{CI} 1.04-1.37)$ among others.

Conclusion: Early post-operative discharge of colorectal cancer patients is increasing despite a lack of improvement in readmission rates and an overall increase in hospitalization costs. Discharging some patients prematurely could lead to readmissions with critical complications related to surgery and increased resource utilization.
Figure 1

Trends in Index Admission and Readmissions for Colorectal Cancer Surgery and Cost of Hospitalization, 2010-2017

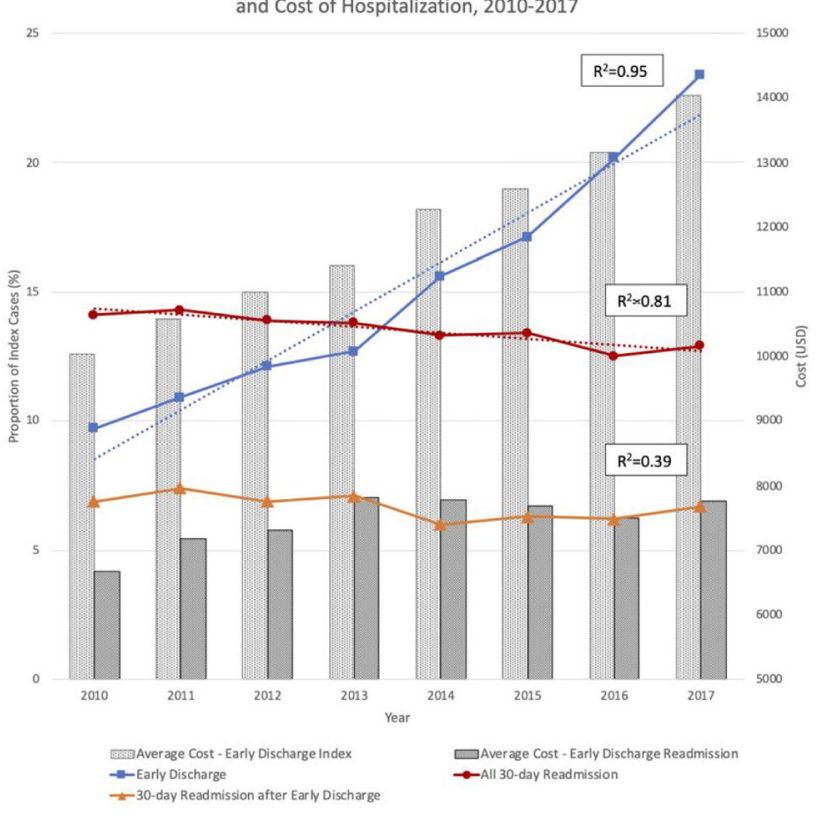


S053

\section{Early ileostomy closure following ileal-pouch anal anastomosis for ulcerative colitis}

Daniel K Peyser, MD; Natalie Berger; Michael Plietz, MD; Anthony P D'Andrea, MD; Sara Lauricella, MD; Gurpawan Kang; Deepika Bhasin, MD; Maia Kayal, MD; Alexander J Greenstein, MD; Sergey Khaitov, MD; Patricia Sylla, MD; Icahn School of Medicine at Mount Sinai.

Background: In patients with ulcerative colitis undergoing ileal-pouch anal anastomosis (IPAA) surgery, construction of a diverting loop ileostomy (DLI) is common. DLI are usually closed $8-12$ weeks postoperatively after confirming anastomotic integrity. DLI result in significant morbidity including dehydration, ileus, and obstruction. Recent studies in rectal cancer have shown that early ileostomy reversal results in fewer complications relative to delayed closure. Data on the optimal timing of ileostomy reversal following IPAA is limited, hence this study aimed to investigate the impact of timing of DLI reversal on postoperative outcomes. Methods: A retrospective analysis of patients who underwent DLI reversal within 12 weeks of IPAA was conducted at a high-volume tertiary referral center. Demographic and postoperative outcomes were analyzed. Patients were classified as either undergoing early ( $\leq 4$ weeks), mid ( $>4-8$ weeks), or late $(>8-12)$ DLI reversal. Data were analyzed using one-way ANOVA test for continuous variables and Chisquare/Fisher exact test for categorical variables.

Results: Among 306 IPAA patients who underwent DLI reversal between April 2008 and October 2020, $15(4.9 \%)$ were reversed early, 109 (35.6\%) mid, and 182 $(59.5 \%)$ late (> 8-12 weeks). Overall complication and readmission rates following IPAA were $41.8 \%$ and $23.2 \%$, of which $86.7 \%$ and $85.9 \%$ were related to the ileostomy respectively. Relative to mid and late DLI reversal, patients reversed early were more likely to have had ileostomy-related complications ( $80 \%$ vs $35.8 \%$ vs $33 \%$ respectively, $\mathrm{p}=0.001)$, at least one readmission $(73.3 \%$ vs $18.3 \%$ vs $16.5 \%$, $\mathrm{p}$ $<0.001)$, or $>1$ readmission $(26.7 \%$ vs $5.5 \%$ vs $6 \%$ ). Among patients reversed early, the majority were reversed during a readmission $(66.7 \%$ vs $6.4 \%$ vs $2.2 \%$, p < 0.001 ). Overall morbidity following reversal was $35.9 \%$ and did not differ between groups, nor did the rate of Clavien-Dindo grade $\geq 3(13.3 \%$ vs $9.2 \%$ vs $6.6 \%)$, anastomotic complications ( $0 \%$ vs $2.8 \%$ vs $3.3 \%)$ or reoperation rates $(13.3 \%$ vs $8.3 \%$ vs $6 \%$ ). While patients undergoing early reversal had longer lengths of stay after reversal ( 7 vs 5 vs 4 days, $\mathrm{p}=0.006)$, there was no significant difference in readmission rates $(13.3 \%$ vs $12.8 \%$ vs $8.8 \%, \mathrm{p}=0.513)$.

Conclusion: Early DLI closure post IPAA was only performed in $4.9 \%$ of patients and mostly prompted by ileostomy-related complications. Early reversal in this complicated setting prolonged hospital stay but did not increase complication rates relative to delayed closure. This data supports exploring the potential role of early DLI reversal in the elective setting to further improve outcomes and quality of life.

\begin{tabular}{|c|c|c|c|c|c|}
\hline & $\begin{array}{l}\text { Overall } \\
n=306\end{array}$ & \begin{tabular}{|l|} 
Early \\
$(\leq 4$ weeks) \\
$n=15$
\end{tabular} & $\begin{array}{l}\text { Mid } \\
(>4-8 \text { weeks) } \\
n=109\end{array}$ & \begin{tabular}{|l|} 
Later \\
(>8-12 weeks) \\
$n=182$ \\
\end{tabular} & $\begin{array}{l}\mathrm{p}- \\
\text { value }\end{array}$ \\
\hline Age (years) & $31(8-83)$ & $27(16-70)$ & $33(13-83)$ & $31(8-74)$ & 0.580 \\
\hline Sex ratio (female : male) & $132: 174$ & $9: 6$ & $43: 66$ & $80: 102$ & 0.302 \\
\hline $\mathrm{BMI}\left(\mathrm{kg} / \mathrm{m}^{2}\right)$ & $22.2(14.2-40.0)$ & $22.4(18.4-28.9)$ & $22.4(15.6-37.3)$ & $22.1(14.2-40.0)$ & 0.661 \\
\hline $\begin{array}{l}\text { All complications (post IPAA+DLL) } \\
\text { lleostomy-related complications }\end{array}$ & $128(41.8 \%)$ & $12(80 \%)$ & $41(37.6 \%)$ & $75(41.2 \%)$ & 0.007 \\
\hline Ileostomy-related complications & $111(36.3 \%)$ & $12(80 \%)$ & $39(35.8 \%)$ & $60(33 \%)$ & 0.001 \\
\hline $\begin{array}{l}\text { All readmissions (post IPAA+DLI) } \\
\text { lleostomy-related readmissions }\end{array}$ & \begin{tabular}{|c}
$71(23.2 \%)$ \\
$61(19.9 \%)$
\end{tabular} & $11(73.3 \%)$ & $21(19.3 \%)$ & $\begin{array}{l}39(21.4 \%) \\
30(16.5 \%)\end{array}$ & $\begin{array}{l}<0.001 \\
<0.001\end{array}$ \\
\hline $\begin{array}{l}\text { Total number of readmissions } \\
1 \\
>1\end{array}$ & \begin{tabular}{|l|}
$98(0.3)$ \\
$50(16.3 \%)$ \\
$21(6.9 \%)$ \\
\end{tabular} & \begin{tabular}{|l|l|}
$16(1.1)$ \\
$7(46.7 \%)$ \\
$4(26.7 \%)$ \\
\end{tabular} & $\begin{array}{l}28(0.3) \\
16(14.7 \%) \\
6(5.5 \%) \\
\end{array}$ & \begin{tabular}{|l|}
$54(0.3)$ \\
$28(15.4 \%)$ \\
$11(6 \%)$ \\
\end{tabular} & $<0.001$ \\
\hline Total readmission LOS (post IPAA+DLL) & $3(1-27)$ & $4(1-9)$ & $3(1-14)$ & $2(1-27)$ & 0.895 \\
\hline $\begin{array}{l}\text { Elective reversal } \\
\text { Reversal during readmission }\end{array}$ & \begin{tabular}{|l|}
$285(93.1 \%)$ \\
$21 / 306(6.9 \%)$
\end{tabular} & \begin{tabular}{|l|}
$5(33.3 \%)$ \\
$10 / 15(66.7 \%)$
\end{tabular} & $\begin{array}{l}102(93.6 \%) \\
7 / 109(6.4 \%)\end{array}$ & $\begin{array}{l}178(97.8 \%) \\
4 / 182(2.2 \%)\end{array}$ & $<0.001$ \\
\hline Time to ileostomy reversal (weeks) & \begin{tabular}{|l|l|}
$2.6(1.9-12.0)$ \\
\end{tabular} & $3.6(1.9-4.0)$ & $6.4(4.4-7.9)$ & $10.0(8.0-12.0)$ & $<0.001$ \\
\hline $\begin{array}{l}\begin{array}{l}\text { Hospital stay after ileostomy reversal } \\
\text { (days) }\end{array}\end{array}$ & $4(1-30)$ & $7(3-21)$ & $5(2-23)$ & $4(1-30)$ & 0.006 \\
\hline $\begin{array}{l}\text { 30-day complication by Clavien-Dindo } \\
\text { grade } \\
\text { Minor (II,II) } \\
\text { Major (III) }\end{array}$ & \begin{tabular}{|l|}
$86(28.1 \%)$ \\
$24(7.8 \%)$
\end{tabular} & $\begin{array}{l}7(46.7 \%) \\
2(13.3 \%)\end{array}$ & $\begin{array}{l}31(28.4 \%) \\
10(9.2 \%)\end{array}$ & $\begin{array}{l}48(26.4 \%) \\
12(6.6 \%)\end{array}$ & 0.871 \\
\hline $\begin{array}{l}\text { Anastomotic complication } \\
\text { Reoperation }\end{array}$ & \begin{tabular}{|l|}
$9(2.9 \%)$ \\
$22(7.2 \%)$ \\
\end{tabular} & \begin{tabular}{|l}
0 \\
$2(13.3 \%)$ \\
\end{tabular} & $\begin{array}{l}3(2.8 \%) \\
9(8.3 \%)\end{array}$ & $\begin{array}{l}6(3.3 \%) \\
11(6 \%)\end{array}$ & $\begin{array}{l}0.760 \\
0.498\end{array}$ \\
\hline 30-day readmission & $32(10.5 \%)$ & $2(13.3 \%)$ & $14(12.8 \%)$ & $16(8.8 \%)$ & 0.513 \\
\hline
\end{tabular}

\section{S054}

Minimally invasive adrenalectomy is associated with equivalent oncologic outcomes among patients with adrenocortical carcinoma

Jonathan J Hue, $\mathrm{MD}^{1}$; Peter Ahorukomeye, $\mathrm{BS}^{2}$; John B Ammori, $\mathrm{MD}^{1}$; Scott M Wilhelm, MD ${ }^{1}$; Luke D Rothermel, MD, MPH ${ }^{1}$; Christopher W Towe, $\mathrm{MD}^{1} ;{ }^{1}$ University Hospitals Cleveland Medical Center; ${ }^{2}$ Case Western Reserve University School of Medicine.

Background: Most data on post-adrenalectomy outcomes for adrenocortical carcinoma (ACC) come from single institutions. We aimed to characterize postoperative outcomes based on operative approach using an administrative dataset. We hypothesized that outcomes of open adrenalectomies (OA) and minimally invasive adrenalectomies (MIA) would be similar, although MIA for ACC is not widely practiced.

Methods: Patients with ACC who underwent adrenalectomy were identified in the National Cancer Database (2010-2016). Patients with clinical nodal or distant metastatic disease were excluded. Resections were classified as OA or MIA. A subgroup analysis was performed among MIA which were converted to open. Operative outcomes were assessed using multivariable regressions. Survival was analyzed using the Kaplan-Meier estimate and multivariable Cox analysis.

Results: 1,041 adrenalectomies were performed: $657(63.1 \%)$ OA and 384 (36.9\%) MIA, of which $286(74.5 \%)$ were laparoscopic and $98(25.5 \%)$ were robotic. Patients who underwent OA were younger ( 55 vs 58 years MIA, $\mathrm{p}=0.01$ ) and had larger tumors (11.0 vs. $7.5 \mathrm{~cm}$ MIA, $\mathrm{p}<0.001)$. There was no difference in margin positivity $(17.4 \%$ OA vs. $21.2 \%$ MIA, $p=0.14)$ or overall survival $(60.7 \mathrm{OA}$ vs. 48.8 months MIA, $\mathrm{p}=0.50$ ) using an intention-to-treat analysis. Cox analysis also showed no survival difference $(H R=1.11, p=0.44)$.

Conversion occurred in 63 MIA procedures (16.4\%). In multivariable modeling, conversion was associated with larger tumors $(\mathrm{OR}=1.07, \mathrm{p}=0.01)$, laparoscopic technique (vs. robotic: $\mathrm{OR}=2.57, \mathrm{p}=0.02)$, and right-sided tumors $(\mathrm{OR}=4.09, \mathrm{p}<$ $0.001)$. Patients who underwent a conversion had a higher rate of positive margins relative to "successful" MIA in univariable $(38.2 \%$ vs. $18.1 \%, \mathrm{p}=0.001)$ and multivariable analysis $(\mathrm{OR}=2.14, \mathrm{p}=0.02)$. Tumor size was not associated with positive margins $(\mathrm{OR}=1.03, \mathrm{p}=0.37)$, but right-sided tumors were associated with an increased risk of positive margins $(\mathrm{OR}=1.85, \mathrm{p}=0.03)$. Surgical approach was not associated with margin positivity (robotic vs. laparoscopic: $O R=0.71, p=0.31$ ).

MIA-conversion was associated with a reduced overall survival compared to "successful" MIA (32.7 vs 56.0 months, p = 0.01, Figure). In multivariable Cox modeling, relative to OA, there was no difference in overall survival among "successful" MIA ( $\mathrm{HR}=0.98, \mathrm{p}=0.90$ ), but conversion was associated with poor survival $(\mathrm{HR}=1.89, \mathrm{p}=0.004)$.

Conclusion: Although OA is considered the gold standard for ACC, survival among patients receiving MIA is similar. MIA-conversion, however, is associated with increased margin positivity and poor survival. MIA may be appropriate for patients with a low risk of margin positivity or conversion, such as small, left-sided tumors. 
S055

\section{Operative Approach and Case Volume are Associated} with Negative Resection Margins for Adrenocortical Carcinoma

Joshua Tseng, MD; Timothy Diperi, MD; Nicholas Gonsalves, MD; Miguel Burch, MD; Daniel Shouhed, MD; Yufei Chen, MD; Anat Ben-Shlomo; Edward Phillips, MD; Monica Jain, MD; Cedars-Sinai Medical Center.

Background: Adrenocortical carcinoma (ACC) is a rare endocrine malignancy with a poor prognosis. Complete surgical resection with negative margins is the treatmen of choice for patients with non-metastatic disease. We sought to determine factors associated with negative resection margins, and whether operative approach affected margin status.

Methods: The National Cancer Database (NCDB) was queried from 2004-2016 to identify all patients with a diagnosis of AJCC/ENSAT Stage I-III ACC who underwent adrenalectomy. Patient, tumor, facility, and operation characteristics were compared by margin status (positive-PM or negative-NM) and by operative approach (open-OA, laparoscopic-LA, or robotic-RA). Multivariate logistic regression was used to identify factors associated with PM.

Results: A total of 1523 patients with non-metastatic ACC who underwent adrenalectomy were identified. PM were reported in $302(19.8 \%)$ patients, and NM were reported in $1221(80.1 \%)$ patients. On univariate analysis, patients with advanced pathologic $\mathrm{T}$ stage and pathologic N1 stage were more likely to have PM (vs NM) (T3, 38.6\% vs $21.8 \%, \mathrm{p}<0.01 ; \mathrm{T} 4,30.5 \%$ vs $11.6 \%, \mathrm{p}<0.01 ; \mathrm{N} 1,10.6 \%$ vs $3.9 \%, \mathrm{p}<0.01)$. There was no significant difference in margin status by tumor size or laterality. Patients undergoing OA (vs LA and RA) were more likely to have advanced clinical T stage (T4, $16.6 \%$ vs $5.7 \%$ vs $7.8 \%, \mathrm{p}<0.01)$ and larger tumors ( $>6 \mathrm{~cm}, 84.6 \%$ vs $64.1 \%$ vs $62.3 \%, \mathrm{p}<0.01)$. High case volume centers $\left({ }^{3} 10\right.$ cases in the study period) were more likely to utilize OA. Patients undergoing LA (vs RA) were more likely to require conversion to open $(20.3 \%$ vs $7.8 \%, p=0.011)$. On multivariate analysis, factors associated with higher odds of PM included T3 disease (OR 6.94, 95\% CI 2.63-18.35), T4 disease (OR 10.26, 95\% CI 3.68-28.67), and LA (OR $1.98,95 \%$ CI 1.27-3.09). High case volume centers were associated with lower odds of PM (OR $0.53,95 \%$ CI $0.32-0.90$ ). There was no significant difference in margin status between OA and RA (OR 1.44, 95\% CI 0.71-2.91). Overall survival was significantly shorter for patients with PM (vs NM) (25.4 vs 78.2 months, p < 0.01 ), even when stratified by stage.

Conclusion: Centers with higher ACC case volumes are more likely to utilize OA in the management of ACC and have lower odds of PM. LA is associated with higher odds of PM and conversion to open, whereas RA is not. These factors should be considered when planning the operative approach for ACC.

\section{S056}

Neuroendocrine Tumors of the Gastrointestinal Tract: A Population-Based Clinical Outcomes Study Involving 57,020 Patients from the Surveillance Epidemiology and End Result (SEER) Database (1975-2016)

Abdul Waheed ${ }^{1}$; Wazir Akbar, MBBS, FCPS $^{2}$; Shaikh A Hai, MD, FACS ${ }^{3}$; Friedrick D Cason, MD, FACS ${ }^{4}$; Veronica Tucci, MD $^{5}$; Ji Fan, MD $^{6}$; Ankita Mishra ${ }^{6} ;$ Kai Huang, $\mathrm{MD}^{6}$; John R Monson, MD, FACS, FASCRS ${ }^{7}$; Bruce Orkin, MD, FACS, FASCRS $^{7}$; Matthew Albert, MD, FACS, FASCRS ${ }^{7}$; Noor Nama, MBBS $^{2}$; Subhasis Misra, MD, FACS ${ }^{6} ;{ }^{1}$ University of South Florida; ${ }^{2}$ Bolan Medical College Quetta, Pakistan; ${ }^{3}$ East Florida Division GME Consortium, Westside Regional Medical Center, Plantation, FL, USA; ${ }^{4}$ San Joaquin General Hospital, French Camp, California, USA; ${ }^{5}$ Oakhill Hospital, Florida, USA; ${ }^{6}$ Brandon Regional Hospital, Brandon, Florida, USA; ${ }^{7}$ Center for Colon and Rectal Surgery, AdventHealth Central Florida Division.

Introduction: Gastrointestinal neuroendocrine tumors (GI-NETs) are a slowgrowing, heterogeneous group of tumors with inconsistent clinicopathologic, biologic, and prognostic characters. Most of the available data regarding this rare entity is limited to a few case series and only a few organ-specific population-based studies. To determine the demographic, clinical, and pathologic factors that impact prognosis and survival, the current study examines the largest ever reported, population-based study of GI-NETs.

Methods: Demographic and clinical data were abstracted from 57,020 patients with GI-NETs from the SEER database $(1975$ - 2016). Statistical analysis was performed with SPSS $\odot$ v20.0 software using the Chi-square test, paired t-test, multivariate analysis, and Kaplan-Meier functions.

Results: The most common primary tumor sites were small intestine $(34.5 \%)$, rectum $(30.6 \% \%)$, stomach $(11.5 \%)$, appendix $(9.8 \%)$, cecum $(4.8 \%)$, and other $(8.8 \%), p<0.001$. Overall, GI-NETs had a higher incidence amongst females $(51.2 \%$ females vs.48.8\% males, $\mathrm{p}<0.001)$ and Caucasians $(76.4 \%, \mathrm{p}<0.001)$ Histologically, the majority of GI-NETs were well-differentiated. However, the tumor of the esophagus (E) (73.2\%), ascending colon (AC) (49.9\%), hepatic flexure (HF) $(58.8 \%)$, transverse colon (TC) $(57.8 \%)$, splenic flexure (SF) $(52.7 \%)$, and descending colon (DC) (42.\%), were more likely to be poorly differentiated. Most GI-NETs had carcinoid histology (low-grade NET) except for tumors of the E, AC, $\mathrm{HF}, \mathrm{TC}$, and SF, which were more likely to be neuroendocrine carcinomas. The majority of GI-NETs presented with either localized or regional spread and had a size of $<2 \mathrm{~cm}$. Tumors of the E, AC, HF, TC, and DC were often larger $(>4 \mathrm{~cm})$ and more likely to present with distant metastasis. Most GI-NETs patients were treated with surgery $(75.1 \%)$ while fewer patients received chemotherapy $(6.2 \%$, mostly esophageal NETs), and radiation (neoadjuvant $=0.19 \%$, adjuvant $=0.67 \%$ ) $p$ $=<0.001$. The 5-year overall cumulative survival rate and 5-year survival after surgery $(91.6 \% \pm 0.3$ and $94.2 \% \pm 0.4$, respectively) was highest in patients with rectal NETs, while it was lowest $(11.1 \pm 2.5 \%$ and $24.0 \% \pm 12.7 \%)$ in patients with esophageal NETs. The Cox model multivariate analysis identified older age, esophageal NETs, tumors $>4 \mathrm{~cm}$ in size, and distant metastasis to be independently associated with increased mortality.

Conclusion: GI-NETs are rare malignancies, most commonly affecting Caucasian females in the small intestine, rectum, stomach, and appendix, with a locoregional distribution and tumor size of $<2 \mathrm{~cm}$. The addition of chemotherapy and radiation appeared to provide no added survival benefits compared to surgical resection alone. 


\section{RISK FACTORS ASSOCIATED WITH VENOUS THROMBOEMBOLISM IN LAPAROSCOPIC SURGERY IN NON-OBESE PATIENTS WITH BENIGN DISEASE}

Daiji Kano, $\mathrm{MD}^{1}$; Chengcheng $\mathrm{Hu}, \mathrm{PhD}, \mathrm{MS}^{2}$; Caitlin J Thornley, $\mathrm{MD}^{1}$; Cecilia Y Cruz, BS $^{3}$; Nathaniel J Soper, MD, FACS ${ }^{3}$; Jennifer F Preston, MD, FAC$\mathrm{S}^{3} ;{ }^{1}$ Phoenix Integrated Surgical Residency; ${ }^{2}$ University of Arizona Mel and Enid Zuckerman College of Public Health-Phoenix; ${ }^{3}$ University of Arizona College of Medicine - Phoenix.

INTRODUCTION: The objective of this study was to demonstrate whether intraoperative positioning is a risk factor for venous thromboembolism (VTE) in nonobese patients undergoing surgery for benign disease. Few studies to date have focused on intraoperative positioning as a risk factor for VTE despite venous stasis being a known risk factor for VTE. The combined effects of vasodilation from general anesthesia, pneumoperitoneum, operative duration and positions that place the legs in a dependent position may contribute to venous stasis. Therefore, we theorized that the reverse-Trendelenburg position specifically would increase the risk of postoperative VTE.

METHODS AND PROCEDURES: Clinical data from 409,629 subjects undergoing laparoscopic surgery in the 2015-2018 NSQIP database were included. Those undergoing oncologic procedures, diagnosed with disseminated cancer, and with BMI $\geq 30$ were excluded. The subjects were divided into three groups based on surgical position (reverse-Trendelenburg (RT), supine (S), and Trendelenburg (T)). The primary outcome was VTE within 30 days postoperative. Secondary outcome was mortality within 30 days.

RESULTS: The RT, S, and T groups consisted of $118,385,66,573$, and 224,671 subjects, respectively. Of the subjects, $44 \%$ were female and $56 \%$ were male, and $48 \%$ of the procedures were performed on inpatients. Overall median BMI was 25.7, and $83 \%$ of subjects were non-smokers. The primary outcome of VTE was seen in $0.26 \% \mathrm{RT}, 0.23 \% \mathrm{~S}$, and $0.49 \% \mathrm{~T}(\mathrm{p}<0.0001)$; mortality within 30 days was $0.34 \%$ $\mathrm{RT}, 0.25 \% \mathrm{~S}, 0.31 \% \mathrm{~T}(\mathrm{p}=0.0033)$. After adjusting for potential risk factors, RT position was associated with a lower VTE risk (OR 0.876 with $95 \%$ CI $0.714,1.07$ ) compared to S; and T had a higher risk (OR 1.12 with $95 \%$ CI $0.925,1.35$ ) though none of these differences reached statistical significance. VTE risk was significantly different across the three groups $(p=0.001)$. There was an increased risk of VTE in inpatient vs outpatient procedures (OR 3.24 with $95 \%$ CI: $2.78,3.78$ ). There was also an increased risk of VTE with increasing operative time (4th quartile (>120 min) vs 1st quartile (<42 $\mathrm{min})$ OR 3.54 with $95 \%$ CI $2.87,4.36)$.

CONCLUSION(S): This study demonstrated that inpatient procedures and longer operative times were associated with increased risk of VTE in laparoscopic surgery performed for benign disease in non-obese patients. While the VTE risk was significantly different across the three positioning groups and RT positioning had a lower risk, there was not a statistically significantly different risk of VTE between the groups.

\section{S058}

A comparison of bilateral axillo-breast approach (BABA) robotic and open thyroidectomy for papillary thyroid cancer after propensity score matching

Se Hyun Paek; Hyungju Kwon; EUMC.V

Background: We investigated the potential advantages of bilateral axillo-breast approach (BABA) robotic thyroidectomy over conventional open surgery through comparing the safety and completeness of surgical procedures.

Methods: We retrospectively reviewed the records of 315 consecutive patients (robotic, $\mathrm{n}=54$; open, $\mathrm{n}=261$ ) who underwent total thyroidectomy and central neck dissection (CND) for PTC from March 2013 to June 2014. Postoperative complication rate and surgical completeness were analyzed between robotic group and open group after propensity score matching.

Results: Transient hypoparathyroidism was higher in the open group than in the robotic group ( 13.0 vs. $1.9 \% ; \mathrm{p}=0.029)$. No difference was observed in the mean number of retrieved lymph nodes (LNs), metastatic LNs, the level of stimulated thyroglobulin (Tg)

Conclusions: Our results show that the outcomes of BABA robotic thyroidectomy can be comparable to those of conventional open thyroidectomy, with possibly better preservation of blood supply to the parathyroid glands.

\section{ROBOTIC VERSUS LAPAROSCOPIC MEDIAN ARCUATE LIGAMENT RELEASE: A RETROSPECTIVE COMPARATIVE STUDY}

Thomas H Shin, MD, PhD; Bradley Rosinski, MD; Andrew Strong, MD; Hana Fayazzadeh, MD; Alisan Fathalizadeh, MD; John Rodriguez, MD, FACS; Kevin ElHayek, MD, FACS; Cleveland Clinic.

INTRODUCTION: Multiple retrospective studies have demonstrated the safety and feasibility of laparoscopic median arcuate ligament division with celiac ganglionectomy for definitive management of median arcuate ligament syndrome (MALS). This study queries the clinical equipoise of robotic (RMALS) versus laparoscopic MAL release (LMALS) at a high-volume center.

METHODS AND PROCEDURES: A retrospective analysis of consecutive 26 RMALS and 24 LMALS between March 2018 and August 2019 by a single surgeon at a quaternary academic institution was completed. Primary endpoint was postoperative decrease in celiac trunk expiratory peak systolic velocities (PSV) measured by mesenteric duplex ultrasonography. Secondary outcomes included reported improvement in MALS-related clinical symptoms, distribution of first assistant seniority level, and involvement of second assistants in RMALS versus LMALS RESULTS: Both groups provided an equally effective decrease in postoperative PSV (LMALS $p=0.0011 ;$ RMALS $p=0.0022 ;$ LMALS vs. RMALS $p=0.7772$ ) There were no significant differences in postoperative symptom relief between groups. Patients with RMALS reported significant relief of chronic abdominal pain ( $<0.0001)$, postprandial abdominal pain $(\mathrm{p}<0.0001)$, and chronic nausea $(\mathrm{p}=$ $0.0002)$. RMALS had significantly more junior first assistants $(\mathrm{p}=0.0001)$ and less frequently required second assistants $(\mathrm{p}=0.038)$ compared to LMALS. Mean operative times for LMALS and RMALS were 86 and 134 min, respectively ( $p<$ 0.0001 ). There were no open conversions and mean length of hospital stay was 1 day for both cohorts.

CONCLUSIONS: In this first study comparing RMALS to LMALS, postoperative outcomes were equivalent, with significantly longer operative time, more junior firs assistants, and fewer second assistants in the RMALS group. Both approaches are safe and feasible for well-selected patients in experienced centers.
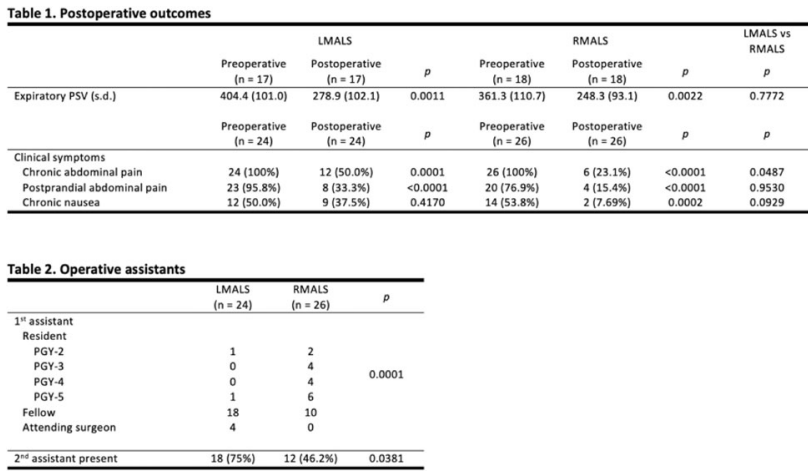
S060

\section{Public and Provider Perceptions of Robotic-Assisted Surgery}

Brian E Walkowski, MD ${ }^{1}$; Meaghan Marley, $\mathrm{MD}^{2} ;{ }^{1}$ Brown University; ${ }^{2}$ Conemaugh Memorial Medical Center.

Introduction: Robotic assisted surgery has seen wide-spread adoption over the past two decades by hospital systems, providers, and patients alike but relatively little is known about how robotic surgery is perceived by these different cohorts.

Objective: The aim of this study was to determine the public's perception of robotic-assisted surgery (RAS) as it pertains to clinical outcomes, provider technical ability, marketability within a community, and the role of robotic surgery in the future. These perceptions were then compared to those held by providers to help identify misperceptions and opportunities for education.

Methods: Questionnaires were distributed to the public within the forum of a community hospital based general surgery clinic and electronic questionnaires were distributed to providers via institutional email accounts. Providers for this study were defined as physicians, physician assistants, nurse practitioners and registered nurses. Questionnaires were collected from December 2019-May 2020 and only those questionnaires with $100 \%$ completion were included and submitted for statistical analysis.

Results: 278 completed surveys were collected and analyzed (162 public and 113 provider). Public respondents were $57 \%$ male and $41 \%$ female, $30 \%$ were 66 years of age or older, and 53\% had a high school diploma or equivalency or less. Provider respondents were $22 \%$ male and $77 \%$ female, $44 \%$ were aged $18-35$, and $88 \%$ had at least a 4 year college degree. $30 \%$ of the public at least slightly agreed with the statement that robots would completely replace surgeons within the next 50 years compared to $10 \%$ of providers $(\mathrm{P}<0.05) .64 \%$ of public respondents at least slightly agreed that surgeons with robotic surgical training are more technically skilled than those that do not compared to $51 \%$ of providers. $41 \%$ of public respondents at least slightly agreed with the statement that robots were able to operate independently of a surgeon compared to $9 \%$ of providers.

Conclusion: There was similarity amongst provider and public perceptions of robotic surgery as they pertained to surgical outcomes, preferred surgical approach, choice of healthcare institutions, and surgical technical ability. However, the public was more likely to perceive robots as operating independently and as having the capability to replace surgeons than their provider counterparts. The perceptions espoused in this study highlight a need for both provider and public education as well as abundant marketing and recruitment opportunities.

\section{S061}

\section{Systematic Intraoperative Assessment of Robotic Technology during High-complexity Surgery}

Mariana J Rodriguez, MD, MPHc ${ }^{1}$; Koen Verkoulen, BS $^{2}$; Michiel Francken, BS $^{2}$; Carlos Cordova, $\mathrm{MD}^{1}$; Kevin Kennedy, $\mathrm{MS}^{1}$; Jose E Gonzalez, $\mathrm{MD}^{1}$; Vitaly Poylin, MD, FACS ${ }^{1}$; Ronald Van Dam, MD ${ }^{2}$; Arthur J Moser, MD, FACS ${ }^{1}$; ${ }^{1}$ Beth Israel Deaconess Medical Center; ${ }^{2}$ Maastricht University Medical Center.

Introduction: Prospective data evaluating the impact of robotic technology on team performance during high-complexity surgery are lacking. We hypothesized that surgical approach and technology affect the frequency and severity of events (flow disruptions, FD) causing cessation of all surgical activity by OR personnel.

Methods: Observational study measuring team performance during open, laparoscopic, and robotic surgery of the liver/pancreas/bile duct (HPB), gallbladder, colon, rectum and prostate at a US academic center. The primary outcome was frequency, duration, and root cause of FDs categorized as interruptions, saves, "near misses", or accidents depending on the control and timespan of remedial activity executed by the OR team. Remediation activity was categorized according to process control, patient benefit, and resiliency. Outcomes were analyzed according to surgical approach (MIS, open); technology (lap, robotic) and emerging (HPB, colorectal) vs. established (prostate) procedures with appropriate controls. Events were recorded by trained, non-expert observers using pre-specified tools.

Results: Observers recorded 3443 FDs spanning 218 cases ( 92 HPB; 105 colorectal; 21 prostate) using laparoscopic $(43 \%)$, robotic $(37 \%)$ or open $(20 \%)$ techniques. The inter-rater Kappa correlation coefficient was $\geq 0.80$. The overall frequency of serious FDs was $9.7 \%$ near misses and $2.1 \%$ saves with one accident. Univariate analysis of the HPB cohort demonstrated increased frequency (7.4 vs $4.8 \mathrm{FD} / \mathrm{hr}$; p < 0.001 ) and FD severity (near miss $16.7 \%$ vs. $11.7 \%$; $=0.006$ ) in the MIS group compared to open, with teamwork (42\%) and technology (18\%) as root causes. After controlling for the MIS approach, FDs occurred with similar frequency/ severity between the robotic and lap HPB groups, with differences in duration attributed to teamwork $(41 \%)$ and teaching $(35 \%)$. Similar effects of the robotic platform were observed during robotic colorectal surgery $(2.9 \mathrm{vs} 2.1 \mathrm{FD} / \mathrm{hr} ; \mathrm{p}=0.003)$ compared to lap right colectomy. When comparing robotic procedures between the three specialties, the robotic HPB group demonstrated both the highest frequency of FD (5.1/ hr vs. 3.8 vs $2.1, \mathrm{p}<0.001)$ and greatest proportion of near misses $(17.8 \%$ vs $12.9 \%$ vs. $8.2, \mathrm{p}<0.001)$ compared to colorectal and prostate surgery, respectively. The root cause of differences was teamwork during HPB and skill acquisition during prostatectomy. Technology had equivalent impact (19\%) between specialties.

Conclusion: These prospective data demonstrate significantly increased frequency and severity of flow disruptions during complex minimally-invasive surgery. The robotic platform amplifies teamwork challenges among OR personnel already managing advanced technology. Widespread implementation (prostatectomy) reduces the adverse impact of robotic technology on team performance, whereas intraoperative teaching amplifies it. 
S062

\section{OUTCOMES FOLLOWING ROBOTIC VERSUS LAPAROSCOPIC SLEEVE GASTRECTOMY: THE NEW YORK STATE EXPERIENCE}

Aaron B Hoffman, MD, FACS ${ }^{1}$; Ajay A Myneni, MBBS, PhD, $\mathrm{MPH}^{1}$; Lorin M Towle-Miller, $\mathrm{MA}^{2}$; Matthew Burstein, $\mathrm{MD}^{1}$; Iman Simmonds, $\mathrm{MD}^{1}$; Steven D Schwaitzberg, MD, FACS ${ }^{1}$; Katia Noyes ${ }^{2} ;{ }^{1}$ University at Buffalo Jacobs School of Medicine and Biomedical Sciences; ${ }^{2}$ University at Buffalo School of Public Health and Health Professions.

INTRODUCTION: The use of robotic platform for bariatric sleeve gastrectomy has gained acceptance and popularity over the last decade. While the evidence on inhospital outcomes and costs associated with robot-assisted (RSG) compared to laparoscopic sleeve gastrectomy (LSG) is consistent, little is known about long-term surgical outcomes ( $>30$ day) in RSG patients. We utilized a large population-based longitudinal dataset to evaluate differences in postoperative outcomes following RSG and LSG.

METHODS AND PROCEDURES: Using 2012-2017 New York State's inpatient and ambulatory surgery data from Statewide Planning and Research Cooperative System, we conducted bivariate and multivariate analyses to examine patient longterm outcomes and postoperative complications following RSG vs LSG in unmatched and propensity score-matched (1512 RSG and 15,120 LSG) samples. We also calculated patient's average hospital charges during admission and up to 1 year from the date of initial surgery.

RESULTS: Among the 58,979 minimally invasive sleeve gastrectomies performed during the study period, $1512(2.6 \%)$ were RSGs. Compared to those undergoing LSG, RSG patients traveled longer distance for surgery and more likely to be covered by private insurance $(66.3 \%$ vs $61.3 \%$ of LSG, p < 0.05$)$. Preoperatively, RSG patients were less likely to have morbid obesity $(97.2 \%$ vs. $98.5 \%$ among LSG patients) and other medical complications and more likely to have substance abuse disorder compared to LSG patients. RSG patients were more likely to have perioperative conversion to open procedure $(3.2 \%$ vs $0.3 \%$ in LSG patients, $\mathrm{p}<0.01)$ and had a longer length of stay ( 2.3 days vs. 1.8 days in LSG patients, $\mathrm{P}<0.01$ ). Rates of postoperative complications were not different between RSG and LSG patients but emergency room visits and inpatient readmissions were more common among RSG patients $(6.0 \%$ vs. 4.5 in LSG patients, $\mathrm{p}<0.05)$. Among super obese $(\mathrm{BMI} \geq 50)$ population, the rates of conversions, inpatient readmissions and length of stay were significantly higher in RSG vs LSG patients. Average hospital charges for the index admission and cumulative changes for 1 year from the date of index surgery were more than $20 \%$ higher for those undergoing RSG.

CONCLUSIONS: Patients undergoing RSG had a higher rate of conversion to open procedures, longer postoperative stay, higher chance of readmissions and higher costs compared to LSG patients. No clear advantages for use of robotic platform for sleeve gastrectomy procedures for either average risk or extremely obese patients were discerned.

\section{S063}

Robotic-Assisted versus Laparoscopic Approach to Concurrent Bariatric Surgery and Hiatal Hernia Repair: Propensity Score Matching Analysis Using the 2015-2018 MBSAQIP

Raul Sebastian, $\mathrm{MD}^{1}$; Jorge Cornejo, $\mathrm{MD}^{1}$; Omar Ghanem, $\mathrm{MD}^{2}$; Thomas Ruttger ${ }^{1}$; Matthew Mayuiers, MD ${ }^{1}$; Christina Li, MD ${ }^{1}$; ${ }^{1}$ Northwest Hospital; ${ }^{2}$ Mayo Clinic.

Introduction: Up to $37 \%$ of severe or morbidly obese patients undergoing bariatric surgery have a hiatal hernia $(\mathrm{HH})$ which is repaired concurrently. Although robotic hiatal hernia repair offers potential technical advantages over traditional laparoscopy, the clinical outcomes of the bariatric surgery and concurrent $\mathrm{HH}$ repair comparing robotic vs laparoscopic approaches have not been reported.

Methods: Using the 2015-2018 Metabolic and Bariatric Surgery Accreditation and Quality Improvement Program (MBSAQIP) database, patients between 18- and 65-years who underwent Sleeve gastrectomy with concurrent HH repair (SG-HHR) or Roux en-Y Gastric Bypass with concurrent HH repair (RYGB-HHR) were identified. Demographic, operative and 30 day-postoperative outcomes data were compared between laparoscopic and robotic groups. To adjust for potential confounders, 1:1 propensity-score matching (PSM) was performed using 22 preoperative characteristics.

Results: 75,034 patients underwent SG-HHR $(n=61,458)$ or RYGB-HHR ( $n=$ 13,576). The operative time was significantly longer in the Robotic-assisted compared to laparoscopic approach either for SG-HHR (102.31 \pm 44 vs. $75.27 \pm 37 ; \mathrm{P}<$ $0.001)$ or for RYGB-HHR $(163.48 \pm 65$ vs. $132.87 \pm 57$; $\mathrm{P}<0.001)$. In the SGHHR cohort (4639 matched cases), the robotic approach showed similar results in 30-day outcomes as in the laparoscopic approach, with no statistical difference. Conversely, for the RYGB-HHR cohort (1502 matched cases), the robotic approach showed significantly fewer requirements for blood transfusions $(0.3 \%$ vs. $1.7 \%$; $\mathrm{P}=$ $0.001)$, fewer anastomotic leaks $(0.2 \%$ vs. $0.8 \% ; \mathrm{P}=0.035)$ and less postoperative bleeding ( $0.4 \%$ vs. $1.1 \%$; $\mathrm{P}=0.049)$.

Conclusion: Robotic bariatric surgery and concurrent $\mathrm{HH}$ repair offer similar overall short-term clinical outcomes despite longer operative times. Furthermore, the robotic approach is associated with significantly reduced blood transfusion requirements and lower incidence of anastomosis leak in the RYGB-HHR group.

\section{S064}

\section{Robotic-Assisted Revision of Biliary Enteric Anastomoses for Benign Biliary Strictures}

Jordan N Robinson, MD, MPH; Christoph Tschuor, MD; Dionisios Vrochides, MD, PhD; John Martinie, MD; Atrium Health.V

Background: Surgical revision of biliary enteric anastomoses is a challenging procedure even for experienced surgeons. Robotic surgery represents an advanced minimally invasive modality with several advantages over both open and laparoscopic approaches. Herein we present a robotic case series of revisions to preexisting biliary enteric anastomoses related to benign strictures with a technical description of how to complete these cases using the robotic platform.

Methods: All patients who underwent robotic revision of benign biliary strictures from November 2012 to August 2020 at Atrium Health, Carolinas Medical Center were reviewed. Details regarding the surgical technique used to revise biliary enteric anastomoses using the robotic platform are reported along with patient demographics and outcomes.

Results: Four patients with benign biliary strictures underwent robotic revision. Ages ranged from thirty-seven to sixty-two years. Average operative time was three hours and thirty-four minutes $(3: 34 \mathrm{~h} \pm 42.71 \mathrm{~min})$. All four patients experienced resolution of their presenting clinical signs and symptoms. Ultimately, two patients were re-admitted, one with a small $(<1 \mathrm{~cm})$ hepatic abscess treated with antibiotics and the second without an identified complication.

Conclusion: Revision of strictured biliary enteric anastomoses using the robotic platform is technically feasible with several potential advantages when compared to open and laparoscopic approaches. Robot-assisted intervention allows the surgeon to perform a minimally-invasive anastomotic revision in a hostile re-operative environment generally associated with high postoperative morbidity. 
S065

\section{Retrospective Analysis of 7 Years of Adrenalectomies and Adoption of the Robotic Technique}

William F Morano; Paul A Vallejos, BS; Alison Dumitriu, MD; Audrey H Choi, MD; Nephtali Gomez, MD; Loma Linda University Health.

INTRODUCTION: Laparoscopy remains the preferred approach to adrenalectomy, demonstrating improved peri-operative results and improved morbidity compared to open technique. Our institution has transitioned to primarily using a robotic approach over the last 10 years.

METHODS AND PROCEDURES: After intuitional-IRB approval, a review of all adrenalectomies (pediatric and adult) from 2013-2020, since introduction of current electronic medical record system, was performed for clinical and pathologic characteristics. After database creation, a statistical analysis was performed. Fisher's exact test was used for proportional analysis and Wilcoxon signed-rank test for continuous variables. Medians and interquartile ranges were also determined.

RESULTS: Since 2013, 85 cases were performed in adult patients and 15 in pediatric patients. Robotic technique has been most commonly performed: 73 robotic, 17 laparoscopic, 10 open. In adults there were 69 robotic, 10 laparoscopic, and 6 open procedures. In pediatric patients there were 4 robotic, 7 laparoscopic, and 4 open procedures. For all patients, median size of lesion was $4 \mathrm{~cm}$ (IQR 3-6). Median length of stay was 2 days (IQR 1-3). Clinical/patient characteristics were similar across the three groups. Median size of lesion was $11 \mathrm{~cm}$ for open versus $4 \mathrm{~cm}$ for both laparoscopic and robotic. Median length of surgery was significantly shorter in the robotic cases as compared to laparoscopy and open (159 vs 207 vs $293 \mathrm{~min}, \mathrm{p}<0.05) .6$ patients had Clavien-Dindo grade 1-2 morbidity after robotic procedure. There was 1 mortality after a laparoscopic procedure. CONCLUSIONS: Robotic approach is a safe and efficient technique for both right and left adrenalectomy. With appropriate learning curve, the length of surgery can be shorter than that of laparoscopy. This approach may allow for comparable length of stay, estimated blood loss, and is an option for use in the pediatric population.

\section{S066}

\section{Single-Port Robotic Resection in Rectal Cancer}

Hyejin Kim; Gyu-Seog Choi; Seung Ho Song; Kyungpook National University, Daegu, Korea.

Purpose: The da Vinci Single-port (SP) system is designed to facilitate singleincision robotic surgery in a narrow space. However, reliable data are currently not available regarding the usefulness of this new system for the treatment of rectal cancer. This study aimed to evaluate the technical feasibility and safety of SP robotic rectal resection for rectal cancer patients based on our initial experience.

Methods: Consecutive patients with mid or low rectal cancer who underwent SP robotic resection between July and September 2020 were reviewed. The demographic characteristics, perioperative data, and pathologic results of the patients were retrospectively analyzed.

Results: Five patients underwent SP robotic rectal resection. The median tumor height from the anal verge was $4 \mathrm{~cm}$ (range, $3-5 \mathrm{~cm}$ ). Two patients received preoperative chemoradiotherapy for advanced rectal cancer. A single docking was conducted, and the median docking time was $4 \mathrm{~min} 20 \mathrm{~s}$ (range, $3 \mathrm{~min} 30 \mathrm{~s}$ to $5 \mathrm{~min}$ ). The median total operation time was $195 \mathrm{~min}$ (range, $155-240 \mathrm{~min}$ ), and the median time of pelvic dissection was $45 \mathrm{~min}$ (range, $36-62 \mathrm{~min}$ ). All patients presented with substantial circumferential and distal resection margins. One patient experienced an anastomosis-related complication. The median duration of hospital stay was 7 days (range, $7-8$ days)

Conclusions: Our initial experience suggests that SP robotic rectal resection is safe and feasible. Further clinical trials comparing SP and multiport robotic rectal resection should be conducted to verify the superior aspects of this new system.

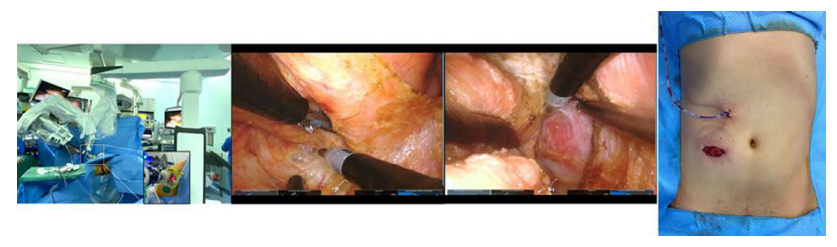

S067

Operating Costs, Fiscal Impact, Value Analysis and Guidance for the Routine Use of Robotic Technology in Abdominal Surgical Procedures

Philippe Quilici, MD; Harry Wolberg, Clinical, Analyst; Nathaniel McConnell; Providence St Joseph Health.

Background: This study was designed to define the value, cost, and fiscal impact of robotic-assisted procedures in abdominal surgery and provide clinical guidance for its routine use.

Methods: 34,984 patients who underwent an elective cholecystectomy, colectomy, inguinal hernia repair, hysterectomy, or appendectomy over a 24-month period were analyzed by age, BMI, risk class, operating time, LOS and readmission rate. Average Direct and Total Cost per Case [ADC, TCC] and Net Margin per Case [NM] were produced for each surgical technique, i.e. open, laparoscopic, and robotic-assisted [RA]. Results: All techniques were shown to have similar clinical outcomes. 9412 inguinal herniorrhaphy were performed [48\% open with \$2,138 ADC, $29 \%$ laparoscopy with $\$ 3,468$ ADC, $23 \%$ RA with $\$ 6,880$ ADC]; 8316 cholecystectomies [94\% laparoscopy with $\$ 2,846$ ADC, $4.4 \%$ RA with a 7,139 ADC, $16 \%$ open with a $\$ 3,931$ ADC]; 3432 colectomies [42\% open with a \$12,849 ADC, 38\% laparoscopy with a $\$ 10,714,20 \%$ RA with a $\$ 15,133$ ]; 12,614 hysterectomies [ $42 \%$ RA with a $\$ 8,213$ OP ADC, 39\% laparoscopy \$5,181 OP ADC, $19 \%$ open $\$ 4,894$ OP ADC. Average Global NM is $-1 \%$ for RA procedures and only positive with commercial payors. Conclusion: RA techniques do not produce significant clinical enhancements than similar surgical techniques with identical outcomes while their costs are much higher. The produced value analysis does not support the routine use of RA techniques for inguinal hernia repair and cholecystectomy. RA techniques for hysterectomies and colectomies are performed at a much higher cost than laparoscopic or open techniques and probably should be routinely used with appropriate clinical justification and by cost-efficient surgical providers.

\section{S068}

Trends and Outcomes of Minimally Invasive Surgery for Gastrointestinal Stromal Tumors (GISTs)

Jonathan Gevorkian, MD; Emily Le; Evan Liggett, MD; Brian Davis, MD; Alan Tyroch, MD; Shintaro Chiba, MD; Ioannis T Konstantinidis, MD; Texas Tech University Health Sciences Center El Paso.

Introduction: Minimally invasive surgery (MIS) is increasingly being utilized for the resection of gastrointestinal cancers. The national trends for perioperative and oncologic outcomes of MIS in Gastrointestinal Stroma Tumors (GIST) are unknown. We hypothesize that with increased use of MIS, the perioperative outcomes and survival for GIST are preserved.

Methods: The National Cancer Database (2010-2016) was utilized to assess perioperative and oncologic outcomes for GIST of the stomach and small bowel.

Results: Data from 8923 gastric and 3683 small bowel resections were analyzed. Over the study period, MIS became the prevalent modality for gastrectomies $(28.5 \%$ in $2010 \mathrm{vs}$ $58.4 \%$ in 2016), with a marginal increase in enterectomies (18.4\% in 2010 vs $31.1 \%$ in 2016). Age and Charlson Comorbidity Index (CCI) were similar amongst groups and CCI was 0 (table). MIS approaches were associated with fewer readmissions and lower 90 day mortality for gastrectomies and similar rates for enterectomies. MIS surgery was also associated with improved median overall survival (table).

Conclusion: Minimally invasive surgery is increasingly being utilized for resection of gastric and small bowel GIST, with improved postoperative outcomes for gastric GIST. Overall survival is not compromised by utilizing MIS for either gastric or small bowel GIST.

Table: Clinicopathologic and Perioperative Outcomes in Robotic (Rob), Laparoscopic (Lap) and Open Approach (Open)

\begin{tabular}{lll}
\hline $\begin{array}{l}\text { Variable, } \mathbf{N} \\
\text { Rob/Lap/Open }\end{array}$ & $\begin{array}{l}\text { Stomach } \\
\mathbf{5 5 6 / 3 5 1 1 / 4 8 5 6}\end{array}$ & $\begin{array}{l}\text { Small Bowel } \\
\mathbf{7 2 / 8 3 9 / 2 7 7 2}\end{array}$ \\
\hline Median Age (years) & $66 / 65 / 66^{*}$ & $63.5 / 63 / 63$ \\
CCI 0 (\%) & $68 / 69 / 69$ & $86 / 74 / 72$ \\
Readmission within 30 days (\%) & $2.2 / 2.1 / 4.1^{* *}$ & $2.8 / 1.2 / 0.1$ \\
90 day Mortality (\%) & $1.5 / 1.3 / 2.9^{* *}$ & $2.1 / 2.4 / 2.8$ \\
Median Survival (months) & $86.2 / 84.3 / 79.1^{* *}$ & $81.8 / 81.7 / 74.5^{* *}$
\end{tabular}

Rob: Robotic, Lap: Laparoscopic, CCI: Charlson Comorbidity Index, *p $<0.05, * * p<$ 0.001 


\section{S069}

The Case for a New Post-Graduate Hernia Specialty Designation: A Review of Fellowship Council Case Logs from the Past Decade

Madhuri B Nagaraj, MD, MS ${ }^{1}$; Adnan Alseidi, MD, EdM ${ }^{2}$; Ajita S Prabhu, MD ${ }^{3}$; Jacob A Greenberg, MD, EdM ${ }^{4}$; Joshua J Weis, MD ${ }^{1}$; Daniel J Scott, MD ${ }^{1}$; ${ }^{1} \mathrm{UT}$ Southwestern; ${ }^{2}$ University of California at San Francisco; ${ }^{3}$ The Cleveland Clinic; ${ }^{4}$ University of Wisconsin School of Medicine and Public Health.

Background: The Fellowship Council is an accreditation body for multiple subspecialty areas. While there has been an increasing emphasis on disease-focused categories, no specific accreditation criteria exist for hernia repairs. The purpose of this study was to analyze the FC case log database for hernia repairs and to determine potential accreditation thresholds for case numbers and surgical approaches.

Methods: De-identified case $\log$ records were obtained from the FC for all hernia cases 2007-2019. Any fellow who had logged $<50$ total cases or no hernia cases were excluded; only one-year programs were included. Cases were coded as either inguinal or ventral hernias, and with or without mesh repair. Retrospective analysis was performed analyzing total hernias logged, type of repair, program designation and robotic adoption. Robotic adoption was categorized by quartiles of program performance in 2018-2019. Statistical analysis was performed using R-Studio®. Results: Over this ten-year period, a total of 93,334 hernia cases were analyzed; included were 5 program designations, 152 unique programs and 1,558 unique fellows. Number of fellows grew from 106 in 2007-2008 to mid-130 s from 2010-2019. Total hernias per fellow increased from 41.2 in 2007-2008 to 75.7 in 2018-2019 (183.7\% growth). Open and robotic hernias increased by $241.9 \%$ and $266.3 \%$ respectively; however, laparoscopic hernias decreased by $14.8 \%$ (Figure). Inguinal and ventral hernias comprised $48.1 \%$ and $51.9 \%$ of total cases respectively. Advanced GI/MIS and Advanced GI/MIS/Bariatrics programs logged the majority of hernias (86.0-90.2\%). A dramatic rise in robotic adoption started in 2014, with fellows averaging $<1$ robotic repair in prior years compared to $>25$ repairs in 2019 . A significant difference was found between all groups when comparing quartiles of robotic adopters (median cases per fellow;IQR): first quartile (78.0;47.9-108.8), second quartile (26.0;21.0-30.6), third quartile (13.0;12.0-14.3) and fourth quartile $(3.5 ; 0.5-5.0)$ (p-value $<0.05)$

Conclusions: Based on this large ten-year analysis, there was a near doubling in the growth of total hernia cases highlighting an opportunity for a hernia specific specialty designation. The decrease in laparoscopic approaches coupled with variable rates of robotic adoption in programs represent a range of hernia training experiences for fellows to choose from as fellowships are established. These data show the importance of hernia repairs in the training of our fellows and warrant further granular analysis to determine specific accreditation criteria for the development of hernia fellowship designations

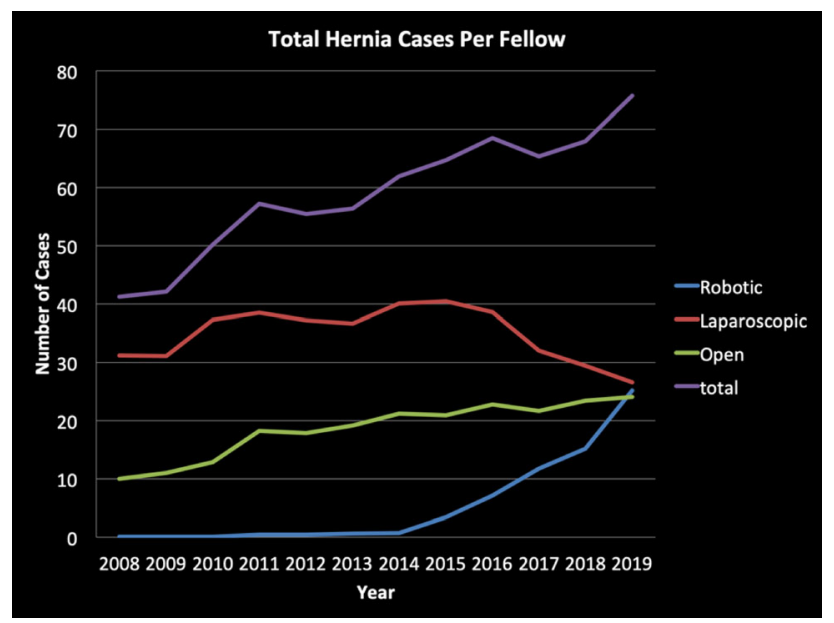

\section{S070}

Financial Incentives to Improve Patient Follow-up after Bariatric Surgery

Grace F Chao, MD, MS ${ }^{1}$; Jeffrey T Kullgren, MD, MPH, MS ${ }^{1}$; Rachel Ross, RN $\mathrm{MS}^{2}$; Aaron J Bonham, $\mathrm{MS}^{2}$; Amir A Ghaferi, MD, MS ${ }^{2} ;{ }^{1}$ Veterans Affairs Ann Arbor; ${ }^{2}$ Michigan Bariatric Surgery Collaborative.

Introduction: Consistent follow-up has the potential to improve weight loss after bariatric surgery and to enable early intervention on post-operative problems. Yet, loss to follow-up is as high as $56 \% 1$ year after bariatric surgery. Given that the goal of bariatric surgery is to help patients achieve weight loss and improvements in obesity-associated comorbidities, we tested whether financial incentives could improve post-surgical patient follow-up and weight loss.

Methods: Using a quasi-experimental design, bariatric surgery patients were invited to participate in a 6-month financial incentives program in three diverse bariatric centers that were part of the Michigan Bariatric Surgery Collaborative (MBSC). In the program, patients received a financial incentive for attending scheduled appointments at 1 month $(\$ 10), 3$ months $(\$ 20)$, and 6 months (\$30) after surgery. At each of the three time points, the incentive was doubled when a participant weighed less at that visit compared to the prior visit. Patients participating in the program were optimally matched with contemporary controls from non-participating MBSC practices by surgery date, age, gender, surgical procedure, pre-surgical BMI, starting weight, and race. Pre-intervention estimates were determined using historic patients selected from intervention practices and non-participating control practices using the same ratio and matching criteria as contemporary controls, but with surgery date only used to match between the two historic groups. We then conducted difference-in-differences analyses to examine the effects of the intervention on the primary outcome of follow-up and the secondary outcome of percent excess weight loss at months 1, 3, 6 and 12 after bariatric surgery.

Results: The 110 participants in the financial incentives program from January 1 , 2018 to July 31, 2019 were matched to 203 patients from the pre-intervention period (November 20, 2017 to December 27, 2017). The control group had 273 patients preintervention and 327 patients post-intervention. Difference-in-differences analysis revealed the intervention increased follow-up rates at 1 month $(+14.8 \%, \mathrm{P}<$ $0.0001), 3$ months $(+29.4 \%, \mathrm{P}<0.0001)$, and 6 months $(+16.4 \%, \mathrm{P}<0.0001)$, but not at 12 months. There were no statistically significant differences in percent excess weight loss at any post-operative time point

Conclusion: Six months of financial incentives of up to $\$ 140$ significantly increased follow-up after bariatric surgery for up to 6 months but did not significantly increase post-operative weight loss. Therefore, our study supports the use of incentivized approaches as one mechanism of improving follow-up which should next be implemented on a broader scale with an evaluation of clinical and healthcare utilization outcomes. 
S071

Ventral hernia management in a bariatric patient population: a summary of treatment recommendations and outcomes

Melissa Hetrick, DO; Sarah Assali, DO; Oliver Knoell, MD; Kellen Hayes, MD; George Eid, MD; Allegheny Health Network.

Introduction: Obesity is a known risk factor for ventral hernia development. Nearly $85 \%$ of patients undergoing ventral hernia repair are overweight (BMI $>25 \mathrm{~kg} / \mathrm{m} 2$ ) and over half are obese (BMI $>30 \mathrm{~kg} / \mathrm{m} 2)$. No consensus exists regarding management of hernias in this patient group. This study reviews treatment recommendations, patient compliance, and outcomes at a multi-surgeon bariatric center.

Methods: This is a retrospective review from May 2015 to December 2019. All patients with obesity who presented with the chief complaint of a ventral hernia, primary and incisional, were included. Initial treatment recommendations were grouped into five categories: (1)staged approach: bariatric surgery preceding hernia repair, (2)concomitant bariatric surgery and hernia repair, (3)medical weight loss (MWL) preceding hernia repair, (4)hernia repair alone, and (5)non-operative management. Patient compliance in each treatment category was assessed. Perioperative outcomes associated with each treatment strategy were evaluated. Additionally, outcomes for patients who only underwent bariatric surgery (as part of a planned staged approach) or required emergent hernia repair were assessed. Hernia recurrence outcomes were evaluated at 1 month, 6 month, 1 year, and 2 year intervals. Results: One hundred and fifty patients met inclusion criteria. The mean \pm SD age and median(IQR) BMI were $55.6 \pm 11.3$ yrs and $41.2(36.3-48.2) \mathrm{kg} / \mathrm{m} 2$, respectively. Most patients were female $(57.3 \%)$ and incisional ventral hernias were the most common hernia type $(76.6 \%)$. Compliance was lowest in the staged group $(11.1 \%)$ and highest in the hernia repair only group $(72.5 \%)(\mathrm{p}<0.001)$. Complications, including wound infection $(72.7 \%$ vs $0 \%, \mathrm{p}<0.001)$ and re-operation $(9.1 \%$ vs $0 \%, p=0.057)$, were highest in patients that required emergent hernia repair and lowest in patients that underwent concomitant bariatric surgery. Length of stay was longer in patients that required emergent hernia repair with a median(IQR) of 5(4-7) days $(\mathrm{p}<0.001)$. Hernia recurrence at two years was lower in patients who underwent a staged approach than those who underwent MWL preceding hernia repair or concomitant bariatric surgery $(\mathrm{p}=0.001)$.

Conclusion: Ventral hernias within the bariatric population are a complex problem to address. Although many treatment strategies exist, in our study, more patients underwent hernia repair when it was performed alone or with concomitant bariatric surgery. Poor compliance was noted in the staged treatment approach, however, this strategy was associated with the lowest perioperative complications and recurrence rates. Complications were expectedly highest in patients that required repair emergently. Efforts to improve compliance with a staged treatment approach should be made to improve hernia repair outcomes and decrease likelihood of recurrence.

\section{S072}

\section{Remote Instruction of Fundamentals of Laparoscopic Skills through Video Streaming}

William Yi, MD; Daria Harlamova; Robert Caskey, MD; Ian Soriano, MD; Victoria Gershuni, MD; Kristoffel Dumon, MD; University of Pennsylvania.

Introduction: The COVID-19 pandemic presents a challenge to the delivery of surgical education. Due to restrictions brought forth by the Centers for Disease Control and Prevention and other organizations, there is an urgent need for developing innovative means of providing surgical instruction—specifically, the ability to teach remotely. The purpose of this study was to determine the feasibility of providing Fundamentals of Laparoscopic Skills (FLS) instruction via a web connected video/audio interface and to show that this instruction is non-inferior to in-person teaching.

Methods: Through the use of simultaneous real-time video streams that captured laparoscopic movements in the operative field, facial expressions, and hand movements, we designed a system of teaching FLS remotely. Educator and learner were able to see how manipulation of a tool directly relates to the operative field while also being able to directly communicate face-to-face. A group of general surgery residents naïve to laparoscopy were randomized into control in-person and experimental remote learning groups. Residents were assessed for time on all 5 FLS tasks and then either given hour long instruction in-person or remotely. All instruction was scripted and provided by the same instructor. Residents had two hours for independent practice and were then tested again. Mean differences in pre- and postinstruction times were compared. We set the non-inferiority margin for the experimental group to the lower bounds of the $95 \%$ confidence interval of the control group.

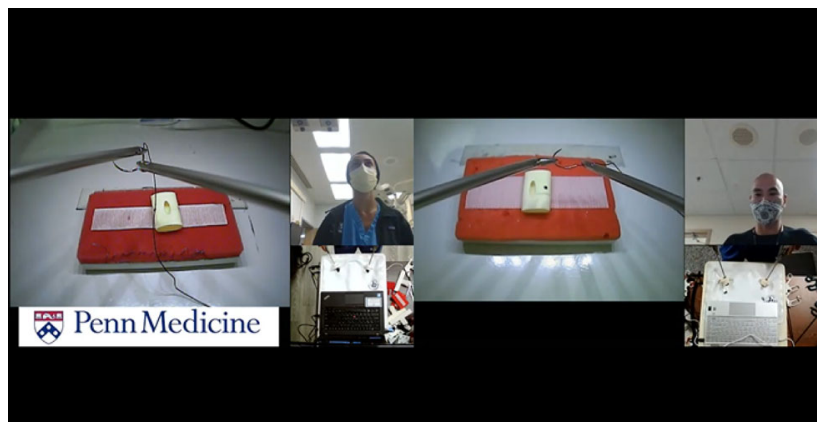

Results: Data collection is ongoing, but as of today, results from a sample of 10 residents have been collected. The mean improvement in time for each task was greater for those receiving remote learning. The lower bounds of the $95 \% \mathrm{CI}$ in terms of time improvement of remote learners was greater than that of in-person learners.

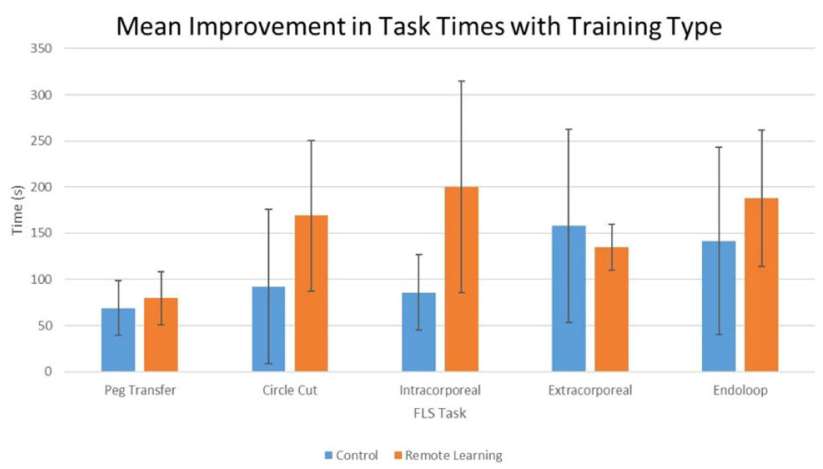

Conclusions: Task completion times improved for all learners on all tasks. For all tasks except extracorporeal tying however, residents showed greater improvements through remote learning, which may be a result of being able to watch both the operator's hands and the operative field at the same time. The lower bounds of the 95\% CI for remote teaching on all tasks was greater than that of in-person teaching, suggesting its non-inferiority. Further data collection may show significantly superior improvements in times with remote learning. 
S073

Follow-up beyond 30 days is required to adequately detect wound outcomes after incisional hernia repair

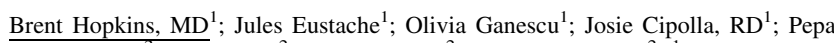
Kaneva, $\mathrm{MSc}^{2}$; JF Fiore $\mathrm{Jr}^{2}$; Lawrence Lee ${ }^{2}$; LS Feldman, $\mathrm{MD}^{2} ;{ }^{1}$ McGill University; ${ }^{2}$ McGill University Health Centre.

Introduction: Incisional hernia repair (IHR) carries a high risk of wound complications. Thirty-day outcomes are frequently used in comparative-effectiveness research, but may miss a substantial number of surgical site occurrences (SSO) including surgical site infection (SSI). The objectives of this study were to determine an optimal length of follow-up to detect SSI after IHR and identify risk factors for SSI in this population.

Methods: All adult patients undergoing open IHR at a single academic centre from 2016 to 2019 were reviewed. The primary outcome was SSI up to 180 days. Secondary outcomes were SSO and hospital readmission. Time-to-event analysis was performed for outcomes at 30,60, 90, and 180 days. Cox-proportional hazards regression was used to calculate the relative hazards of SSI for relevant risk factors. Results: 234 patients underwent open IHR. Median length of documented follow-up 102 days (IQR 37-264 days). Mean age was 58 years, 50.9\% female, $91 \%$ elective, $86 \%$ used mesh, $27 \%$ component separation, and $7.7 \%$ bowel resection. Using the Modified Hernia Grading Scale (MHGS), 32\% were Grade 1, 55\% Grade 2, and 13\% Grade 3. Overall incidence of SSI was $15.8 \%$ with median time to occurrence of 23 days (IQR $12-30$ days). Incidence of non-infectious SSO was $35.5 \%$, and woundrelated readmission was $12.8 \%$. Sensitivity for outcomes at 30,60 , and 90 days are presented in Table 1. After stratifying for technique of component separation and adjusting for hernia grade, smoking exposure (HR 2.76, 95\%CI 1.19-6.39) and emergency surgery (HR 3.20, 95\%CI 1.21-8.47) were independently associated with SSI.

Conclusion: A considerable proportion of SSIs occurred beyond 30 days, but 90-day follow-up detected $92 \%$ of SSIs. These results have implications for the design of trials evaluating wound complication after IHR, as early endpoints may miss clinically relevant outcomes and underestimate the number-needed-to-treat. This study supports the use of a 90-day follow-up for detecting wound complications following IHR

Table 1. Sensitivity of Outcomes with Extended Follow-up

\begin{tabular}{|c|c|c|c|c|c|c|c|}
\hline \multirow[t]{2}{*}{ Outcome } & \multirow{2}{*}{$\begin{array}{l}\text { 180-days } \\
\text { Total events }\end{array}$} & \multicolumn{2}{|c|}{ 30-days } & \multicolumn{2}{|c|}{ 60-days } & \multicolumn{2}{|c|}{ 90-days } \\
\hline & & $\mathrm{n}$ & $\%$ Detected & $\mathrm{n}$ & $\%$ Detected & $\mathrm{n}$ & $\%$ Detected \\
\hline SSI & 37 & 30 & 81.6 & 33 & 89.5 & 34 & 92.1 \\
\hline SSO & 83 & 54 & 65.3 & 77 & 92.7 & 81 & 97.6 \\
\hline Readmission & 30 & 14 & 46.7 & 23 & 76.7 & 25 & 83.3 \\
\hline
\end{tabular}

\section{S074}

Patient-Reported Outcomes Demonstrate Improvement in Both Depression and Satisfaction with Social Roles One Year After Bariatric Surgery

Jessica Blumhagen, MD; Paige Martinez, MS; Eric Volckmann, MD; Anna Ibele, MD; Ellen Morrow, MD, MS; University of Utah.

Background: Bariatric Surgery has the potential to improve quality of life in multiple domains. The Patient-Reported Outcomes Measurement Information System (PROMIS) has validated question banks to assess for Satisfaction with Social Roles (SSR) and Depression.

Objective: To examine changes in Depression and SSR after bariatric surgery, and persistence in changes over a one year period. We also aimed to understand differential effects of bariatric procedures (sleeve gastrectomy (SG) and gastric bypass (RYGB) on these metrics.

Methods: Single academic institution retrospective analysis of prospectively collected PROMIS data from 378 patients who underwent bariatric surgery between March 2015 and January 2019. PROMIS depression and SSR T-scores were measured within six-months prior to surgery date as well as six-months and 12-months post-operatively. Multivariate logistic regression and t-tests were used to compare changes from baseline.

Results: $71.3 \%$ of patients underwent RYGB, and $28.7 \%$ received SG. This bariatric surgery population had less Satisfaction with Social Roles at baseline compared to the US general population mean of 50, which improved to slightly better than average at one-year post-operatively in both RYGB $53.63(\mathrm{SD}=8.85)$ and SG 51.86 $(\mathrm{SD}=9.57)$. Although all SSR scores improved, RYGB patients had greater improvement $($ RYGB SSR $?=12.65$; SG SSR $?=8.84, \mathrm{p}<0.05)$. Depression PROMIS scores improved at six-months and 12-months post-operatively compared to baseline for both groups $(\mathrm{p}<0.001)$

Conclusion: Utilizing validated patient-reported outcomes, bariatric surgery patients demonstrated significant post-operative improvement in both Depression and Satisfaction with Social Roles quality of life measures. This effect was sustained for one year post-surgery. 


\section{S076}

\section{Characterizing Perioperative Costs of Robotic Versus Open Transversus Abdominis Release}

Britta J Han, MD, MSEd; Bradley Kushner, MD; Margaret Sehnert, RHIA; Sara E Holden, MD; Jeffrey Blatnik, MD, FACS; Washington University in St Louis/Barnes Jewish.

Introduction: Transversus Abdominis Release (TAR) is an effective procedure for the repair of complex ventral hernias. The robotic TAR (R-TAR) is a viable minimally invasive alternative to open TAR (O-TAR). However, cost of R-TAR is often a salient point. The aim of this study was to characterize perioperative costs of O-TAR and R-TAR patients.

Methods: Retrospective chart review was completed for patients undergoing O-TAR or R-TAR by two fellowship-trained abdominal wall specialists at an academic medical center from $1 / 2018$ to $2 / 2020$. Similar surgical techniques were utilized. Demographic information was collected including age, sex, BMI, hernia recurrence, hernia defect, co-morbidities, and perioperative clinical data. Exclusion criteria included combined procedures (i.e. gynecological/urological), use of biologic mesh, totally extraperitoneal or pre-peritoneal repairs, and patients without post-operative follow-up. The perioperative cost for each patient was obtained from billing services. Total cost components included room \& board (R\&B), OR cost, ICU, imaging, and physical/occupational therapy (PT/OT). Mann-Whitney U was used to compare the groups; however, means are reported.

Results: Data of 236 patients were analyzed (143 O-TARs; 93 R-TARs). Three patients required conversion to open and were included in the O-TAR group. BMI, Defect size, OR time, and length of stay (LOS) were statistically different (Table 1). The total costs between the groups were not statistically different (O-TAR: $\$ 10,747$ vs R-TAR: $\$ 9,018, \mathrm{p}=0.07)$. However, OR cost of O-TAR was significantly less $(\$ 5,045$ vs $\$ 7,654, p<0.00)$; while $R \& B$ cost of O-TAR was significantly greater ( $\$ 2,779$ vs $\$ 944, \mathrm{p}<0.00)$. Costs associated with admission (lab, PT/OT, pharmacy) were significantly greater in O-TAR patients $(\mathrm{p}<0.00$ for all). The percent of total cost spent on OR was greater for R-TAR patients $(53.4 \%$ vs $85.7 \%, \mathrm{p}<0.00)$ while percent of total cost on R\&B was greater for O-TAR patients $(30.5 \%$ vs $9.5 \%$, p < $0.00)$.

Conclusions: Total cost did not differ between O-TAR and R-TAR. O-TAR cost is primarily incurred from admission while R-TAR cost is primarily incurred from OR. While further study with matched patient sets is warranted, this initial data suggests $\mathrm{R}$-TAR is not cost prohibitive for the repair of complex hernias in the right clinical context.

Table 1. Baseline Demographics

\begin{tabular}{|l|l|l|l|}
\hline & O-TAR (mean) & R-TAR (mean) & p value \\
\hline Age $($ year) & 58.6 & 60.0 & 0.16 \\
\hline BMI $\left(\mathrm{kg} / \mathrm{m}^{2}\right)$ & 32.5 & 30.2 & 0.02 \\
\hline Defect size $\left(\mathrm{cm}^{2}\right)$ & 339.3 & 133.6 & $<0.00$ \\
\hline OR time (minutes) & 319.4 & 307.3 & $<0.04$ \\
\hline Length of stay (days) & 4.8 & 1.9 & $<0.00$ \\
\hline
\end{tabular}

\section{S078}

\section{Endoscopic Versus Laparoscopic Drainage of Pancreatic Pseudocysts: A Cost-Effectiveness Analysis}

Shefali Bansal, BS ${ }^{1}$; Ashley Gallagher, BS$^{2}$; Patrick Quinn, $\mathrm{MD}^{3}$; Ravi Chokshi, MD, MPH, FACS ${ }^{4}{ }^{1}$ Rutgers University Graduate Studies; ${ }^{2}$ Rutgers University School of Medicine; ${ }^{3}$ Ohio State University, Dept of Surgery; ${ }^{4}$ Rutgers University, Surgical Oncology.

Introduction: Pancreatic pseudocysts are a common complication of pancreatitis, resulting from extravasation of pancreatic fluid following damage to the pancreas. Some pancreatic pseudocysts will resolve on their own, nevertheless, a portion of pseudocysts will require intervention due to being symptomatic or causing gastric outlet obstruction. Both endoscopic and laparoscopic drainage are safe and effective approaches, however, the optimal treatment method remains controversial.

Methods: The purpose of our study was to design a decision tree model to analyze both costs and survival in patients with symptomatic pancreatic pseudocysts who require drainage. Patients with infected collections or wall-off necrosis were not included. Within the model, patients were divided into two groups, those that would undergo endoscopic drainage and those that would undergo laparoscopic drainage. Data acquired from the relevant published literature was used to populate the model. Costs were assessed utilizing a third-party payer perspective, specifically Medicare reimbursement rates. Treatment effectiveness was measured in quality-adjusted lifeyears (QALYs) using the declining exponential approximation of life expectancy (DEALE) method based on available morbidity and mortality data. A threshold of $\$ 100,000$ per QALY gained was used to determine the most cost-effective strategy. The model was tested for validation using a one-way, two-way, and probabilistic sensitivity analysis.

Results: Endoscopic drainage of pancreatic pseudocysts was the dominant economic strategy. Endoscopic drainage resulted in 16.32 QALYs at an average cost of $\$ 19,178.61$. Laparoscopic drainage resulted in a decrease of 0.1 QALYs, with an increase in cost of $\$ 23,687.04$. Endoscopic drainage remained the dominant strategy across all one-way sensitivity analyses. Two-way sensitivity analysis demonstrated that a laparoscopic approach is only favored with the combination of high endoscopic acute mortality rate and low endoscopic treatment success. In all other combinations, endoscopic treatment was always favored. Endoscopic drainage was favored in $99.6 \%$ of 100,000 random-sampling simulations.

Conclusion: For patients with non-infected, symptomatic pancreatic pseudocysts, the most cost-effective method of management is with endoscopic drainage. Although laparoscopic surgery can produce better health outcomes, this treatment is double the cost of endoscopic drainage and is well above our $\$ 100,000$ threshold. 
S079

\section{Predicting Early Discharge and Readmission Following Pancreaticoduodenectomy}

Diana S Hsu, MD ${ }^{1}$; Sidney T Le, MD ${ }^{1}$; Kaylin $\mathrm{Cho}^{2}$; George Kazantsev, $\mathrm{MD}^{2}$; Alex $\mathrm{L}$ Chang $^{2}$; Austin Spitzer, $\mathrm{MD}^{2}$; Peter D Peng, $\mathrm{MD}^{2}$; Ching Kuo Chang, $\mathrm{MD}^{2} ;{ }^{1} \mathrm{UCSF}$ East Bay; ${ }^{2}$ Kaiser Permanente Oakland Medical Center.

Introduction: Implementing enhanced recovery after surgery (ERAS) protocols for major abdominal surgery has been shown to decrease length of stay and postoperative complications, including mortality and readmission. Little is known to guide which patients undergoing pancreaticoduodenectomy (PD) should be eligible for ERAS protocols.

Methods and Procedures: A retrospective chart review of all PD performed from 2009-2018 within an integrated healthcare system was conducted. A predictive score that ranges from 0 to 4 was developed, with 1 point assigned to each of the following: obesity (BMI $>30$ ), operating time $>400 \mathrm{~min}$, estimated blood loss (EBL) $>400 \mathrm{~mL}$, low or high risk pancreatic remnant (based on presence of soft gland or small duct). Chi-squared tests and ANOVA were used to assess the relationship between this score and length of stay (LOS), discharge before post-operative day seven, readmission, mortality, delayed gastric emptying (DGE), and pancreatic leak/fistula.

Results: 291 patients were identified. Median length of stay was 8.5 days in those patients who scored 0 compared to 16.2 days for those who scored 4 ( $\mathrm{p}=0.001$ ). $30 \%$ of patients who scored 0 were discharged before postoperative day 7 compared to $0 \%$ of those who scored $4(\mathrm{p}=0.019)$. Readmission rates for patients who scored 0 and 4 were $12 \%$ and $33 \%$, respectively $(\mathrm{p}=0.017)$. Similarly, postoperative pancreatic fistula occurred in $2 \%$ versus $25 \%$ in these groups $(\mathrm{p}=0.007)$.

Conclusion: A simple scoring system using BMI, operating time, EBL, and pancreatic remnant risk can help risk-stratify post-operative PD patients, and patients with lower scores could potentially be managed via an ERAS protocol. Patients with higher scores require longer hospitalizations, and adjunctive therapy, such as medication and surgical technique to decrease risk of delayed gastric emptying and pancreatic fistula, could be considered.

\begin{tabular}{|c|c|c|c|c|c|c|}
\hline ERAS score & 0 & 1 & 2 & 3 & 4 & \\
\hline $\mathrm{n}$ & 60 & 99 & 76 & 44 & 12 & 291 \\
\hline LOS & 8.5 & 10.2 & 10.7 & 10.2 & 16.2 & $P=.001$ \\
\hline DC before 7 days & $30 \%$ & $14 \%$ & $16 \%$ & $18 \%$ & $0 \%$ & $P=.019$ \\
\hline Re-Admission & $12 \%$ & $13 \%$ & $21 \%$ & $23 \%$ & $33 \%$ & $P=.017$ \\
\hline Mortality & $0 \%$ & $1 \%$ & $1.30 \%$ & $4.50 \%$ & $0 \%$ & $P=.359$ \\
\hline DGE & $12 \%$ & $19 \%$ & $18 \%$ & $18 \%$ & $25 \%$ & $P=.771$ \\
\hline Pancreatic fistula & $2 \%$ & $15 \%$ & $12 \%$ & $20 \%$ & $25 \%$ & $P=.007$ \\
\hline
\end{tabular}

S080

\section{IMPORTANCE OF LYMPH NODE 8A AND POOR PROGNOSIS IN PANCREATIC CANCER: A NOVEL APPROACH}

Danny Conde Monroy, MD, MSc, General Surgery ${ }^{1}$; Carlos Rey, MD ${ }^{1}$; Felipe Giron, MD, MsC, Biomedical Engineer'; Manuel Pardo' ${ }^{2}$ Andrea Recaman ${ }^{2}$; Carmen Santos García ${ }^{2}$; Juan Sabogal Olarte, MD, MSc, Hepatobiliary and Pancreatic Surg ${ }^{1} ;{ }^{1}$ Hospital Universitario Mayor Mederi; ${ }^{2}$ Universidad el Rosario.

Introduction: Pancreatic cancer occupies the 7th place worldwide in cancer related deaths. Surgical management followed by adjuvant oncologic management is considered the gold standard, however just $12-18 \%$ achieve 5 years survival. Nonetheless there is still controversy around prognostic factors including hepatic artery lymph (HLN) node metastasis (known as lymph node 8A), impact on disease free survival time (DFS) and overall survival time (OS). Therefore, in the present study, we want to determine the impact of HLN (Lymph Node 8A) metastases in overall and disease-free survival time.

Objective: To evaluate the prognostic significance of HLA (Lymph node 8A) node metastasis in overall and disease-free survival time in patients that underwent pancreaticoduodenectomy (PD) in a referral and high volume centre in Bogotá, Colombia.

Methods: A retrospective observational cohort study of a prospective database was conducted. Included patients underwent a pancreaticoduodenectomy for malignancy between 2014 - 2019 in a single institution by a single HPB surgeon in Bogotá, Colombia. Overall survival and disease-free survival time were estimated by Kaplan Meier analysis. Log-rank test and multivariate cox proportional hazards regression were used.

Results: A total of 102 patients underwent PD resection, 84 patients met inclusion criteria. Mean age was 63,11 years old. The total patients underwent standard NPPD (100\%). $21.43 \%$ of the patients showed metastatic disease in HLN. Mean number of harvested lymph nodes was 14.84 (SD 5,8). Ductal adenocarcinoma was reported in $63,10 \%$. Ampullary carcinoma was $21.43 \%$, and $5.95 \%$ for cholangiocarcinoma. Mean lymph node ratio was 0,45 (SD 0.05). OS in the positive HLN group was 12.5 months versus 23.16 in the negative group. (CI 95\% 12-22). DFS in HLN + group was 13 months, versus the negative group of 20 months. (CI 95\% 26; 22). Survival analysis for positive HLN node and lymph node ratio (LNR) $>0.6$ show a strong relationship with OS (Cox Regression p 0.03 and 0.003 respectively CI 95\%). In terms of DFS, only the LNR shows relationship with the outcome, with a significant statistical value. (p 0.008 CI 95\%).

Conclusion: In our series of pancreaticoduodenectomy of Pancreatic Cancer, HLA (Lymph Node 8A) metastases should be considered as an important prognostic factor in patients with pancreatic malignancy due to its statistical and clinical impact on overall survival and DFS. However, further studies have to be performed to confirm our results. 
S081

Neuroendocrine Tumors of the Gallbladder: A Population-Based Clinical Outcomes Study Involving 325 Patients from the Surveillance Epidemiology and End Result (SEER) Database (1975-2016)

Abdul Waheed ${ }^{1}$; Wazir Akbar, MBBS, FCPS ${ }^{2}$; Shaikh A Hai, MD, FACS ${ }^{3}$; Veronica Tucci, MD $^{4}$; Friedrick D Cason, MD, FACS ${ }^{5}$; John R Monson, MD, FACS, FASCRS $^{6}$; Bruce A Orkin, MD, FACS, FASCRS ${ }^{6}$; Matthew R Albert, MD, FACS, FASCRS $^{6}$; Noor Nama, MBBS ${ }^{2}$; Subhasis Misra, MD, FACS ${ }^{7} ;{ }^{1}$ University of South Florida; ${ }^{2}$ Bolan Medical College Quetta, Pakistan; ${ }^{3}$ East Florida Division GME Consortium, Westside Regional Medical Center, Plantation, FL, USA; ${ }^{4}$ Oakhill Hospital, Florida, USA; ${ }^{5}$ San Joaquin General Hospital, French Camp, California, USA; ${ }^{6}$ Center for Colon and Rectal Surgery, AdventHealth Central Florida Division; ${ }^{7}$ Brandon Regional Hospital, Brandon, Florida, USA

Introduction: Gallbladder Neuroendocrine tumors (GBNETs) are rare neoplasms, accounting for $0.5 \%$ of all neuroendocrine tumors, as well as $<2 \%$ of all gallbladder carcinomas. Most of the literature related to this rare disease's biological behavior is limited to case reports and small case series. To the best of our knowledge, the current study represents the largest cohort of GBNETs so far. It aims to examine a large group of patients with GBNETs to determine demographic, clinical, and pathologic factors that influence patients' prognosis and survival with this rare condition.

Methods: Data of 325 patients with GBNETs was abstracted from the Surveillance Epidemiology and End Result (SEER) database (1975 - 2016). Chi-square test, paired t-test, multivariate analysis was used to analyze the data.

Results: GBNETs had higher incidence amongst females $(67.7 \%, \mathrm{p}<0.005)$, Caucasians $(79 \%, \mathrm{p}<0.005)$, and married patients $(50 \%, \mathrm{p}<0.005)$. Histologically, the majority of GBNETs in the database had an unknown grade $(52.3 \%)$, while poorly differentiated tumors were $(20 \%)$, followed by well-differentiated $(14.5 \%)$, undifferentiated $(8.6 \%)$, and moderately differentiated $(4.6 \%), \mathrm{p}=<0.005$. Most GBNETs were $<2 \mathrm{~cm}$ in size $(21.5 \%)$ and localized $(29.8 \%), \mathrm{p}=<0.001$. Most GBNETs patients were treated with surgery $(65.8 \%)$, while fewer patients received radiation $(5.5 \%)$ and chemotherapy $(22.8 \%), \mathrm{p}=<0.001$. The 5-year overall survival was $45.3 \% \pm 3.5$. Those who received surgery, radiation, and chemotherapy had a 5- year survival of $70.8 \% \pm 6.5,42.9 \% \pm 18.7$, and $45.50 \% \pm 11.2$, respectively, $\mathrm{P}=0.037$. Multivariate analysis identified older age $(\mathrm{OR}=2.9, \mathrm{CI}=$ 2.4-2.9), married status $(\mathrm{OR}=2.3, \mathrm{CI}=1.6-2.1)$, large tumor size $(>4 \mathrm{~cm}),(\mathrm{OR}=$ $1.9, \mathrm{CI}=1.2-1.5)$, Caucasian race $(\mathrm{OR}=2.2, \mathrm{CI}=1.6-2.4)$, and metastasis at presentation $(\mathrm{OR}=1.7, \mathrm{CI}=1.1-1.2)$ to be independently associated with increased mortality, $\mathrm{p}<0.001$

Conclusion: GBNETs are rare malignancies, mostly affecting Caucasian female patients after the 5th decade of life. The combination of chemotherapy and radiation appeared to provide no added survival benefits compared to surgical resection alone.

\section{S082}

Promoting a Culture of Safety in Cholecystectomy (COSIC) over a decade at a Philippine public regional hospital after the SAGES International Proctoring Course in Laparoscopic Cholecystectomy Course

William Yi, MD ${ }^{1}$; Juan Esteban Perez, MD ${ }^{1}$; Claire Rosen, $\mathrm{MD}^{1}$; Ferdauzi Akalal, MD, FPCS, FPSGS, FPALES ${ }^{2}$; Alex Cerillo, MD, FPCS, FPSGS, FPALES ${ }^{2}$; Anam Alawaddin, MD, FPCS, FPAHPBS, FPALES ${ }^{2}$; Ibrahim Hassan, MD²; Joji Sese, MD, FPCS, FPSPS ${ }^{2}$; Ian Soriano, MD, FACS, FASMBS, FPALES, Hon ${ }^{1} ;{ }^{1}$ University of Pennsylvania; ${ }^{2}$ Zamboanga City Medical Center.

Background: The SAGES International Proctoring Course for Laparoscopic Cholecystecomy accepts applications from low- and middle-income countries for SAGES faculty to train local surgeons. A regional public hospital in the 10th most populous city in the Philippines was one of the chosen sites for the 1-week course in 2010. Two SAGES surgeons and one nurse trained two local surgeons and four nurses identified by the hospital director.

Methods: All patients seen in the out-patient clinic and scheduled for elective laparoscopic cholecystectomy from the first day of the course in August 2010 until December 2019 were entered into a prospectively collected database including demographics, pre-op diagnosis, operative findings, histopathologic diagnosis, conversion rates and 30-day complications including re-operations.

Results: 652 patients were entered into the database. Majority were female $(63.7 \%)$ with a mean age of 45.9 years. Most procedures were completed laparoscopically with an open conversion rate of $3.6 \%$. Three patients underwent laparoscopic subtotal cholecystectomy. Reported complications requiring reoperation included one stump necrosis, 2 incisional hernias and one retained stone. One serosal injury and one surgical site infection were also reported for an overall morbidity rate of $3.4 \%$. Pathology showed chronic calculous cholecystitis in $92.8 \%$ of specimens. No 30-day mortality was recorded.

Conclusion: The SAGES International Proctoring Course for Laparoscopic Cholecystectomy has been shown to be a successful method for global surgery training. A focused 1-week direct proctoring model in the Philippines showed a sustained Culture of Safety in Cholecystectomy with low 30-day morbidity, complication and conversion rates over a decade following participation in this program. 
S083

\section{MULTICENTRIC VALIDATION OF A COMPUTER VISION PLATFORM TO VIDEO DOCUMENT THE CRITICAL VIEW OF SAFETY IN LAPAROSCOPIC CHOLECYSTECTOMY}

Pietro Mascagni ${ }^{1}$; Deepak Alapatt ${ }^{2}$; Giovanni G Laracca ${ }^{3}$; Ludovica Guerriero ${ }^{4}$; Andrea Spota ${ }^{3}$; Claudio Fiorillo ${ }^{1}$; Armine Vardazaryan ${ }^{2}$; Elisa Cassinotti ${ }^{6}$; Luigi Boni $^{6}$; Diego Cuccurullo ${ }^{4}$; Guido Costamagna ${ }^{1}$; Bernard Dallemagne ${ }^{7}$; Nicolas Padoy $^{2} ;{ }^{1}$ Fondazione Policlinico Universitario A. Gemelli IRCCS, Rome, Italy; ${ }^{2}$ ICube, University of Strasbourg, CNRS, IHU Strasbourg, France; ${ }^{3}$ Department of Medical Surgical Science and Translational Medicine, Sant'Andrea Hospital, Sapienza University of Rome, Italy; ${ }^{4}$ Department of Laparoscopic and Robotic General Surgery, Monaldi Hospital, AORN dei Colli, Naples, Italy; ${ }^{5}$ Scuola di Specializzazione in Chirurgia Generale, University of Milan, Italy; ${ }^{6}$ Department of Surgery, Fondazione IRCCS Ca' Granda Ospedale Maggiore Policlinico di Milano, University of Milan, Italy; ${ }^{7}$ Institute for Research against Digestive Cancer (IRCAD), Strasbourg, France.

INTRODUCTION: A computer vision(CV) platform was recently developed to facilitate the use of videos for surgical education, documentation and research. This $\mathrm{CV}$ platform was tested in its ability to provide short video clips documenting the critical view of safety(CVS) in laparoscopic cholecystectomy(LC). The aim of the present study is to validate the CV platform on a multicentric dataset of LCs.

METHODS: 125 LC videos were collected from 4 centers. The CV platform leveraged predictions of deep learning models for surgical phase recognition and tool detection in a rule-based inference system designed to estimate the time of the cystic duct division. Performance was assessed by computing the error in estimating the manually annotated time of the cystic duct division. To provide concise video documentation of CVS, the CV platform automatically extracted clips showing the $2 \mathrm{~min}$ preceding and the $30 \mathrm{~s}$ following the cystic duct division. The relevance of the documentation was evaluated by surgeons assessing CVS in the short videos.

RESULTS: The CV platform located the time of the cystic duct division with a mean error of $126.8 \pm 284.7 \mathrm{~s}$ despite fluorescent cholangiography in 27 procedures and great variations in surgical workflows (Table 1). The surgical evaluation found that $97(77.6 \%)$ of the automatically extracted 2:30 min long clips documented CVS effectively.

CONCLUSIONS: The CV platform was robust enough to reliably locate the time of the cystic duct division and efficiently video document CVS despite the highly variable LC workflows. Fine-tuning on data from each center and selecting larger video clips will improve results, however, this multicentric validation shows the potential for clinical translation of this surgical data science system to promote surgical safety.

CV platform performance stratified by center

\begin{tabular}{|c|c|c|c|c|c|}
\hline & $\begin{array}{l}\text { Internal } \\
\text { testing }\end{array}$ & Gemelli & Sant'Andrea & Ca'Granda & Monaldi \\
\hline $\begin{array}{l}\text { Analyzed } \\
\text { LC[total] }\end{array}$ & 100 & $7[11]$ & $24[29]$ & $56[64]$ & $38[49]$ \\
\hline $\begin{array}{c}\text { Mean error } \pm \\
\text { standard } \\
\text { deviation } \\
\text { (seconds) }\end{array}$ & $62.8 \pm$ & 130.4 & $40.6 \pm 21.4$ & $113.9 \pm 151.8$ & $\begin{array}{l}121.0 \pm \\
337.8\end{array}$ \\
\hline \multicolumn{6}{|l|}{$\begin{array}{r}159.3 \pm \\
292.5\end{array}$} \\
\hline \multicolumn{6}{|l|}{$\begin{array}{l}\text { no[\%] videos }^{\circ} \text { with errors }\end{array}$} \\
\hline$<30 \mathrm{~s}$ & $66[66 \%]$ & $2[28.6 \%]$ & $11[45.8 \%]$ & $32[57.1 \%]$ & $7[18.4 \%]$ \\
\hline $30-60 \mathrm{~s}$ & $9[9 \%]$ & $3[42.9 \%]$ & $1[4.2 \%]$ & $11[19.6 \%]$ & $8[21.1 \%]$ \\
\hline $1-2 \min$ & $12[12 \%]$ & $2[28.6 \%]$ & $5[20.8 \%]$ & $3[5.4 \%]$ & $11[28.9 \%]$ \\
\hline $2-5 \min$ & $8[8 \%]$ & $0[0 \%]$ & $4[16.7 \%]$ & $4[7.1 \%]$ & $6[15.8 \%]$ \\
\hline$\geq 5 \min$ & $5[5 \%]$ & $0[0 \%]$ & $3[12.5 \%]$ & $6[10.7 \%]$ & $6[15.8 \%]$ \\
\hline
\end{tabular}

\section{S085}

EVALUATION OF THE KNOWLEDGE OF THE CRITICAL VIEW OF SAFETY AND RECOGNITION OF THE TRANSOPERATIVE COMPLEXITY DURING THE LAPAROSCOPIC CHOLECISTECTOMY

Bianca Alanis-Rivera, MD ${ }^{1}$; Gabriel Rangel-Olvera, MDMSc ${ }^{2} ;{ }^{1}$ Hospital de Especialidades Dr. Belisario Domínguez / Hospital Central de Alta Especialidad Pemex; ${ }^{2}$ Hospital General "Dr Manuel Gea Gonzalez"/ Hospital General Milpa Alta.

Introduction: Since the establishment of the critical view of safety (CVS) for cholecystectomy in 1995; different strategies like bailout procedures (subtotal cholecystectomy, $\mathrm{SC}$ ), classifications for the preoperative and intraoperative complexity (like the Parkland Grading Scale,PGS) and the objective evaluation of the CVS (Doublet score,DS) have been created to stablish a "Culture of Safety of Cholecystectomy"; with the main objective to avoid complications like the bile duct injury (DBI) and clarifying the different steps to follow during the cholecystectomy and the different scenarios that this procedure can present. The aim of the study is to evaluate the knowledge of the CVS, PGS, SC, DS and the preferred action in different surgical scenarios.

Methods: A multiple choice questionnaire was applied to residents and surgeons from different Hospitals in Mexico City and during different national meetings; the results were presented by frequencies, range and means. Univariate logistic regression was used to evaluate the knowledge of the different concepts with the referral of having presented a BDI.

Results: A total of 744 questionaries were evaluated; $284(38.17 \%)$ referred to be female and 460(61.83\%) male; $436(58.6 \%)$ were residents and 308(41.4\%) graduated surgeons with a mean experience of 11.97(1-59)years. 500(67.2\%) mentioned to work mainly in public institutions, $200(26.88 \%)$ in private and $44(5.91 \%)$ in both. 708(95.16) mentioned to know the CVS; however only 368(49.46\%,p $=<0.001)$ chose the correct definition of it, also $136(18.28 \%)$ referred to know the DS, but only 44 correctly defined the DS. About the PGS, 398(53.49\%) mentioned to know the scale, but only 262 correctly defined it. $642(86.29 \%)$ referred to know the concept of SC; however only $(48.92 \%, \mathrm{p}=<0.001)$ correctly defined the techniques, being the reconstituting technique the preferred one $(272,42.37 \%$ VS $224,34.89 \%)$ $338(45.43 \%)$ referred to leave a drainage routinely and $66(8.87 \%)$ referred to use routinely IOC. $138(18.55 \%)$ referred to have presented a BDI. On this survey the correct knowing of the CVS (OR 0.47,p $<0.001)$, of the subtotal techniques (OR $0.71, \mathrm{p}=0.07)$, of the DS (OR 0.48,p $<0.001)$ and of the PGS (OR 0.12,p $<0.001)$ appear to be a protective factor for presenting BDI.

Conclusion: Despite the culture of safety cholecystectomy and the time since the CVS was published; the percentage of people who are able to correctly define basic safety concepts is low among the residents and graduated surgeons. Therefore, it is important to emphasize the dissemination of these concepts to obtain safe LC and thus have an impact on the incidence of complications such as BDI 


\section{S088}

The rise and fall of percutaneous cholecystostomy in a community hospital: is it possible to turn the tide of history?

Alexander Ostapenko; Shawn Liechty; Stephanie Stroever; Daniel Kleiner; Danbury Hospital.

Introduction: Laparoscopic cholecystectomy is the gold standard for treatment of patients with cholecystitis. Percutaneous cholecystostomy (PC) drains have been utilized as an alternative to surgery in critically ill patients in whom surgery carries an unacceptably high risk of morbidity and mortality. Though initial use of the procedure was primarily as a temporizing measure for patients recovering from an acute illness, the use of PC drainage has been rising. However, this practice has recently been challenged by the CHOCOLATE trial, which demonstrated a significant increase in complications in patients undergoing percutaneous drainage in comparison to those undergoing laparoscopic cholecystectomy.

Methods: We conducted a retrospective analysis of patients who underwent PC drainage or a cholecystectomy from 2014 through 2019 at our institution. We determined the rate of PC use and performed a linear regression analysis to determine if the use of percutaneous drainage has been increasing over time. In 2019 we reeducated resident and faculty on appropriate PC use and compare the rate of PC in 2019 to prior years.

Results: A total of 148 PCs and 3,163 cholecystectomies were performed over the study period. Of the cholecystectomies, $754(23.8 \%)$ were performed in hospitalized patients under urgent or emergent condition. The percentage of patients with emergent cholecystitis that were treated with PC was $16.4 \%$. Linear regression analysis demonstrated a statistically significant association between year and proportion of PC procedures performed $(\mathrm{p}<0.001)$. We observed a gradual increase in the use of PC between 2014 and 2018 from 14.5 to $22.5 \%$. In 2019 a reeducation program along with formal policy regarding PC was instituted, after which the percentage of PCs dropped to $10.9 \%$. This was accomplished without an increase in common bile duct injuries.

Conclusions: Although the rate of PC drains at our institution is consistent with those reported in the literature, we observe a steady rise in their use despite a relatively stable rate of cholecystectomies. This would suggest that though the rate of biliary disease remains consistent, PCs are being selected as the procedure of choice with increasing frequency. Care needs to be taken to ensure that only appropriate patients are referred for percutaneous drainage, as indicated by the guidelines, especially in light of recent studies suggesting an increase in complications as well healthcare costs for patients undergoing PCs compared to laparoscopic cholecystectomy.

\section{S089}

AGARENA Study: Antibiotic timing in gangrenous appendicitis

Manuela Pineda, $\mathrm{MD}^{1}$; Anibal Ariza, $\mathrm{MD}^{1}$; Juan $\mathrm{F}$ Infante ${ }^{1}$; Jairo Mahecha ${ }^{1}$ Alfonso Marquez, MD ${ }^{1}$; Carlos E. E Diaz-Castrillon ${ }^{2} ;{ }^{1}$ Pontificia Universidad Javeriana; ${ }^{2}$ University of Pittsburgh Medical Center.

INTRODUCTION: Standardizing postoperative care aids in delivering efficient therapeutic strategies while expecting reproducible clinical outcomes; however, when it comes to antibiotic therapy in complicated appendicitis, there is still some debate in the literature regarding the appropriate postoperative antibiotic duration within this heterogeneous population. We aim to describe the gangrenous appendicitis population and evaluate the standardization of $24 \mathrm{~h}$ of intravenous antibiotic therapy as a quality improvement policy in our hospital.

METHODS: A single-center retrospective study at a University-based Hospital including all consecutive patients who underwent an appendectomy (open or laparoscopic) with intraoperative findings of gangrenous appendicitis (macroscopic signs of necrosis or gangrene without perforation) between January 2014 and December 2019. Patients under 18 years, pregnant women, or receiving antibiotics for other causes were excluded.

All patients received preoperative antibiotic prophylaxis. The extent of peritoneal reaction, as well as the duration of postoperative antibiotic treatment, was recorded. The primary endpoint was the surgical site infection (SSI) rate and reintervention rate. Secondary outcomes were the length of hospitalization, readmission; and 30-day mortality rate.

RESULTS: A total of 3,914 patients were admitted to our institution with a diagnosis of appendicitis, out of which $13.7 \%$ had intraoperative signs of gangrenous appendicitis. After the exclusion of 55 patients, 486 remained eligible for analysis. The median age was 35 years (IQR 26-51) with $43.1 \%$ female patients and $74.1 \%$ categorized as ASA I. Overall, only $1 \%, 1.8 \%$, and $3.1 \%$ reported chronic corticoid utilization, diabetes mellitus or an immunosuppressive state, respectively. The median preoperative white blood cell count was 16,000 (IQR 13,000-19,000) and $87.7 \%$ of cases were performed laparoscopically. The median hospital stay was 2 days (IQR 2-3), where $67.4 \%$ of the patients received only $24 \mathrm{~h}$ of postoperative intravenous antibiotic therapy. Patients with longer $\mathrm{AB}$ therapy had more peritoneal reaction $(\mathrm{p}<0.05)$. Overall SSI rate was $4.5 \%(\mathrm{n}=22)$, with $68 \%(\mathrm{n}=15)$ being intraabdominal abscess. The rate of ISS among $24 \mathrm{~h}$ IV group was $3 \%$ vs $7.5 \%$ for the $>24$ h group $(p=0.02)$. Moreover, reintervention rate was $0.9 \%$ for $24 \mathrm{~h} \mathrm{AB}$ group vs $4.4 \%$ for the $>24 \mathrm{~h}$ group $(\mathrm{p}=0.01)$. No mortality at 30 days was registered.

CONCLUSION: Postoperative antibiotic treatment after a timely surgical procedure is determined by the extent of contamination and inflammation within the abdominal cavity. In patients with signs of gangrenous appendicitis without extensive peritoneal reaction, the utilization of $24 \mathrm{~h}$ of intravenous antibiotics seems to be safe in our institution. 
S090

\section{Timing is Everything: Outcomes of 30,044 Delayed} Cholecystectomies in New York State

Andrew Guenthart, $\mathrm{MD}^{1}$; Nina Devas, $\mathrm{BS}^{1}$; Gareth Morris-Stiff, $\mathrm{MD}^{2}$; Lizhou Nie, $\mathrm{MSc}^{1}$; Isha Joshi, $\mathrm{BA}^{1}$; Jie Yang, $\mathrm{PhD}^{1}$; Aurora Pryor, $\mathrm{MD}^{1}$; ${ }^{1}$ Stony Brook University Hospital; ${ }^{2}$ Cleveland Clinic.

Introduction: Laparoscopic cholecystectomy is the recommended intervention for acute cholecystitis, however the timing of cholecystectomy in relation to outcomes has been debated. While there is strong evidence to show early laparoscopic cholecystectomy (48-72 $\mathrm{h}$ post admission) leads to lower morbidity, mortality, and hospital costs, it is unclear if beyond this time there is a linear relationship of decreasing outcome with increasing time. To our knowledge, there are no large population-based studies looking at outcomes and complications of delayed cholecystectomy $[\mathrm{DC}]$ ( $>72 \mathrm{~h}$ after presentation). This study utilizes a statewide database to determine whether there are differences in patient outcomes for DC performed at 3-4 days, 5-6 days, and $\geq 7$ days after presentation.

Methods: The New York SPARCS database was used to identify adult patients presenting with a diagnosis of acute cholecystitis from 2005-2017, excluding 2013 due to missing procedure-date information. Patients aged $<18$, those with missing identifier or procedure-date information, and in-hospital deaths were excluded. Patients undergoing DC at 3-4 days, 5-6 days, and $\geq 7$ days were compared in terms of overall complications, hospital length of stay (LOS), 30-day readmissions/ emergency department (ED) visits, and 30-day mortality. Multivariable logistic regression models were used to compare complications, 30-day readmissions/ED visits, and 30-day mortality, after adjusting for patients' characteristics, surgery type, and comorbidities. Forward selection was applied for 30-day mortality because the event rate was too low. Multivariable generalized linear regression for a negative binomial distributed count data was used to compare LOS.

Results: 30,044 patients were identified. DCs were performed within 3-4 days ( $\mathrm{n}=$ $19,759,65.8 \%), 5-6$ days $(n=6393,21.3 \%)$, and $\geq 7$ days $(n=3892,13 \%)$. There was a stepwise deterioration in outcomes with increased delay to surgery (Fig. 1). When comparing 3-4 and $\geq 7$ days, overall complications $(\mathrm{OR}=0.418,95 \% \mathrm{CI}$ : $0.387-0.452), 30$-day readmissions ( $\mathrm{OR}=0.609,95 \%$ CI: 0.549-0.674), 30-day ED visits $(\mathrm{OR}=0.697,95 \% \mathrm{CI}: 0.637-0.763), 30$-day mortality $(\mathrm{OR}=0.601,95 \% \mathrm{CI}$ : $0.400-0.904)$, and $\operatorname{LOS}(\mathrm{OR}=0.729,95 \% \mathrm{CI}: 0.710-0.748)$ were lower in the 3-4 day cohort.

Conclusion: DC within 3-4 days is associated with fewer complications, readmissions and ED visits, and reduced LOS compared to DC at 5-6 or $\geq 7$ days after presentation. In addition, 30-day mortality was also significantly different comparing 3-4 with $\geq 7$ day cohorts. This data is important for guiding patients in the consent process and may point to choosing an interval cholecystectomy for high-risk patients.

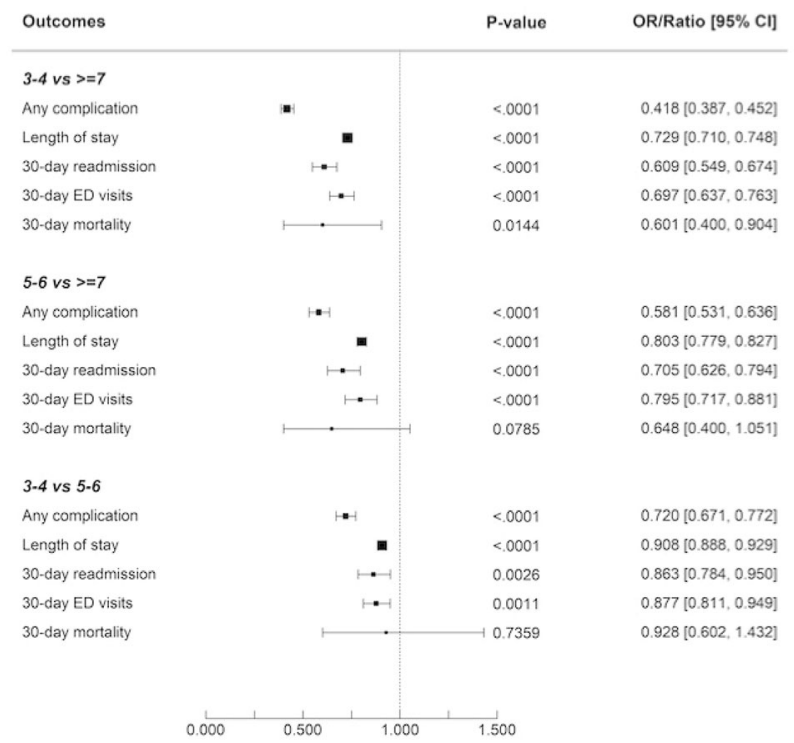

Figure 1 . Forest plot comparing 3-4 day and 5-6 day cholecystectomy timing groups with the $\geq 7$ day group in terms of all studied outcomes.
S091

The NOpioid Project: A qualitative investigation of non-narcotic post-operative pain control

Joseph N DeVitis, MD ${ }^{1}$; Emily Flom, $\mathrm{MS}^{2}$; Tristan Cooper-Roth, $\mathrm{MS}^{3}$; Alan Davis, $\mathrm{PhD}^{1}$; Laura Krech, $\mathrm{PhD}^{1}$; Douglas Kwazneski, $\mathrm{MD}^{1}$; Alistair Chapman, $\mathrm{MD}^{1}$; Carrie Valdez, $\mathrm{MD}^{1}$; ${ }^{1}$ Spectrum Health/Michigan State University; ${ }^{2}$ Michigan State University CHM; ${ }^{3}$ Michigan State University COM.

Background: Prescription of opioids after surgical procedures has contributed to the rise in opioid and substance use and has fueled an increase in opioid associated morbidity and mortality. Alternative means of post-operative pain control via non-opioid pharmaceuticals helps to avoid side effects of opioids while using medications with a lower risk profile. We investigated a non-opioid based post-operative pain management protocol after laparoscopic appendectomies and laparoscopic cholecystectomies.

Methods: The acute care surgical group at a tertiary referral center examined patients from October 2019 to October 2020 undergoing urgent laparoscopic appendectomy and laparoscopic cholecystectomy. Inclusion criteria included ages $18-65$, no prior history of chronic pain or narcotic use, no contraindications to taking Tylenol or ibuprofen, and a GFR $>60$. Patients were allowed narcotics both preand post-operatively while inpatient. Pre- and post-operative counseling was provided to each patient about non-narcotic based pain control. Patients were not prescribed narcotics at discharge and were instead prescribed ibuprofen and acetaminophen. Patients were surveyed approximately two weeks post-operatively at their clinic appointment to assess pain control and other patient reported outcomes including quality of life (QOL; high, moderate/fair, poor).

Results: A total of 51 patients were identified who met the inclusion criteria and completed the postoperative survey. There were 32 females $(62.8 \%)$, and the age was $37.8+14.3$ years $($ mean $+\mathrm{SD})$, with a BMI of $30.4+7.9$. Thirty $(58.8 \%)$ underwent laparoscopic appendectomy for acute non-perforated appendicitis and $21(41.2 \%)$ underwent laparoscopic cholecystectomy for acute cholecystitis. Most patients $(88.2 \%, \mathrm{n}=45)$ were satisfied with their post-operative pain control. Thirty-four patients $(66.7 \%)$ rated their QOL after surgery as high, while $17(33.3 \%)$ rated QOL as moderate/fair. None of the respondents rated their QOL as poor. A majority of patients $(66.7 \%, \mathrm{n}$ $=34$ ) had fascial suture closure of one port site. Pre-existing comorbid conditions associated with increased opioid use, including anxiety, depression and/or alcohol use were not associated with decreased pain control or QOL. There were no significant differences between the appendectomy and cholecystectomy groups with regards to QOL or satisfaction with pain control $(\mathrm{p}>0.05)$.

Conclusion: The opioid epidemic continues to be an urgent public health issue. Our study demonstrates a post-operative multimodal pain control strategy that does not include narcotics on discharge and provides adequate pain control for patients undergoing laparoscopic appendectomy and laparoscopic cholecystectomy. This study presents a high-volume opportunity for surgeons to impact opioid use. Future studies should focus on expanding this non-narcotic pain approach to other procedures. 
S092

Does the Implementation of an Emergency General Surgery Model Reduce Costs Associated with Cholecystectomies

Daniel H Wolbrom, MD, MPH; M. Tyler Guinn, PhD; Lizhou Nie, MS; Jie Yang, PhD; Konstantinos Spaniolas, MD; Salvatore Docimo, DO; Amy L Rosenbluth, MD; Kinga Powers, MD, PhD; Mark A Talamini, MD, MBA; Aurora D Pryor, MD, MBA; Samer Sbayi, MD, MBA; Stony Brook University Renaissance School of Medicine.

Introduction: Emergency general surgery (EGS) services are a novel effort towards providing streamlined and specialized care to patients presenting with acute care surgery needs. While the literature demonstrates improvements in length of stay, morbidity, readmission, and other measures of outcomes with implementation of an EGS model, there is little evidence of improvements in overall charges. We sought to evaluate the overall charges associated with cholecystectomies at a single, academic institution before and after the implementation of an EGS model.

Methods: A longitudinal, observational study was performed to evaluate the influence of the EGS service on the total charges for cholecystectomies at a single, academic institution. Using the New York SPARCS Administrative Database, all adult patients that presented to the emergency department and underwent a cholecystectomy were identified. The charges associated with cholecystectomies 3 years prior (Pre) and 3 years following (Post) the implementation of the EGS service were analyzed and compared to similar institutions in our region. Total charge is defined as all hospital-based charges billed for, and does not include physician fees. Total charge was compared using generalized linear models for a negative binomial distributed outcome, after adjusting for significant risk factors in corresponding univariate regression models.

Results: There were 866 cholecystectomies performed at our institution in the 6-year study period, 4,754 performed in similar academic institutions and 15,809 performed in community institutions, in our region. For the 3 Pre years, our institution saw no statistical difference in total charges when compared to similar academic institutions in the region $(\mathrm{RR}=1.04,95 \%$ C.I. 0.99, 1.08). Furthermore, for the 3 Pre years, our institution reported higher total charges when regional community institutions were compared with our institution $(\mathrm{RR}=0.89,95 \%$ C.I $0.85,0.93)$. Post EGS, total charges were lower at our institution when other regional academic institutions were compared with our institution $(R R=1.20,95 \%$ C.I. 1.15 , 1.25). Finally, our institution also reported lower total charges Post EGS compared to community institutions when comparing regional community institutions with our institution ( $\mathrm{RR}=1.06,95 \%$ C.I. $1.02,1.11)$

Conclusion: The implementation of the EGS model at our academic institution is associated with lower total charges compared with regional academic or community hospitals for all patients treated with cholecystectomy.

\section{S093}

\section{IMPACT ON INFECTIOUS OUTCOMES DURING LAPAROSCOPIC CHOLECYSTECTOMY WITH THE USE OF HOME MADE VS COMMERCIAL GALLBLADDER RETRIEVAL BAG: A RETROSPECTIVE COMPARATIVE STUDY IN A HIGH VOLUME CENTER}

Luis Felipe Cabrera Vargas, MD; Camilo Andres Avella Molano, MD; Francisco Alejandro Rincón Comba; Roosevelt Fajardo Gómez, MD, MBA, FACS; Francisco Javier Díaz Cuervo, MD; Fundación Santa Fe de Bogotá.

INTRODUCTION: Laparoscopic cholecystectomy (LC) is one of the most commonly performed emergency procedures, with approximately 600,000 patients undergoing the procedure every year in the United States. Although LC is associated with fewer complications when compared with open cholecystectomy, the risk for infectious complications, including surgical site infection and intra-abdominal abscess, remains a significant source of postoperative morbidity. The goal of this study is to determine whether the gallbladder retrieval technique during LC affects the risk of infectious complications.

METHODS AND PROCEDURES: We conducted a retrospective comparative study in a minimally invasive surgery high volume center in Bogota, Colombia Patients who underwent LC from 2018 to 2020 were identified. The patients were divided into three groups. One group of LC performed using a home-made gallbladder retrieval bag (HMGRB), another group of LC performed using a commercial gallbladder retrieval bag (CGRB), and another group as control with no use of retrieval bag (NRB). The primary outcomes were infectious complications of superficial site infection and intra-abdominal abscess.

RESULTS: A total of $68(6.18 \%)$ patients underwent LC using an HMGRB, 829 (75.36\%) using a CGRB, and $203(18.45 \%)$ without any bag. There was no significant difference in preoperative sepsis or Parkland Grading Scale severity between patient groups. Using ANOVA we found differences in surgical times $(p=<0.01)$, length of stay $(p=0.02)$, and use of postoperative use of antibiotics $(p=0.04)$. Nonetheless, there was no difference in the rate of superficial surgical site infection $(\mathrm{p}=0.46)$ between groups nor Drain use $(\mathrm{p}=0.08)$.

CONCLUSION: HMGRB is not associated with an increased risk of postoperative intra-abdominal abscess or superficial surgical site infection in comparison with CGRB, but implies longer surgical times and length of stay. The use of HMGRB is safe, feasible, and has a lower cost during LC.

\begin{tabular}{|c|c|c|c|c|c|}
\hline & & \multicolumn{3}{|c|}{ RETRIEVAL BAG } & \multirow[b]{2}{*}{$p$ value } \\
\hline & & HOME MADE & COMMERCIAL & NO BAG & \\
\hline \multicolumn{2}{|c|}{ SURGICAL TIME } & & & & $<0,01$ \\
\hline & & $83,3(31-238)$ & $60,69(18-238)$ & $57,84(22-203)$ & \\
\hline \multirow[t]{6}{*}{ PARKLAND } & & & & & 0,1235 \\
\hline & I & 19 & 392 & 107 & \\
\hline & II & 3 & 67 & 10 & \\
\hline & III & 20 & 202 & 57 & \\
\hline & IV & 12 & 78 & 10 & \\
\hline & V & 14 & 90 & 11 & \\
\hline \multirow[t]{3}{*}{ DRAIN USE } & & & & & 0,08 \\
\hline & No & 55 & 759 & 190 & \\
\hline & Yes & 13 & 70 & 5 & \\
\hline \multicolumn{2}{|c|}{ SURGICAL SITE INFECTION } & & & & 0,4679 \\
\hline & No & 68 & 827 & 195 & \\
\hline & Yes & 0 & 2 & 0 & \\
\hline \multicolumn{2}{|c|}{ LENGHT OF STAY (Hours) } & & & & 0,021 \\
\hline & & $37,75(3-397)$ & $28,55(0-276)$ & $22,95(2-285)$ & \\
\hline \multicolumn{3}{|c|}{ POSTOPERATIVE USE OF ANTIBIOTICS } & & & 0,0489 \\
\hline & No & 23 & 556 & 152 & \\
\hline & $<24$ hours & 17 & 92 & 19 & \\
\hline & 1-3 days. & 4 & 17 & 2 & \\
\hline & 4-7 days & 15 & 94 & 13 & \\
\hline & $>7$ days & 7 & 44 & 3 & \\
\hline
\end{tabular}


S094

\section{The Challenging Small Bowel Obstruction: Frailty in the Elderly}

Franchesca Hwang, MD, MSc ${ }^{1}$; Marie Crandall, $\mathrm{MD}^{2}$; Niel Parry, $\mathrm{MD}^{3}$; Alan Smith, $\mathrm{PhD}^{1}$; Amy E Liepert, $\mathrm{MD}^{1}$; ${ }^{1}$ University of California San Diego; ${ }^{2}$ University of Florida; ${ }^{3}$ London Health Sciences Centre.

Background: Small bowel obstruction (SBO) is a common and frequently morbid disease that affects all segments of population, including frail older patients. As the geriatric population is growing across the United States, older patients are increasingly undergoing surgery. The management of SBO has evolved over time, from mandatory operative intervention to operation guided by gastrointestinal contrast studies, though recent retrospective data suggest that earlier operative intervention may decrease morbidity. However, management decisions are often influenced by patient characteristics and surgical outcomes. Our goal was to determine the current surgical management of SBO, with particular attention to the frail older patients.

Study Design: After IRB approval, we performed a retrospective review of all patients over the age of 65 with a diagnosis of bowel obstruction (ICD-10 K56*) using the 2016 National Inpatient Sample (NIS). Demographics including age, race, insurance status, medical comorbidities, and median household income by zip code were analyzed. Elixhauser co-morbidities were used to derive a previously published frailty score using the NIS dataset. Outcomes included time to operative intervention, mortality, discharge disposition, and hospital length of stay. Descriptive statistics, bivariate analyses, multivariable logistic and linear regression models were calculated to determine the associations between demographics, frailty, and outcomes.

Results: 492,030 patients met the inclusion criteria. Six percent of the cohort was frail. The overall mortality was $4.1 \%$ and the frail had 1.97 increased odds of mortality (95\% CI 1.78-2.17). Hospital length of stay was twice as long for frail patients, and only one third of the frail were discharged home. Patients transferred from another facility had increased mortality (aOR 1.76; 95\% CI 1.56-1.99). Independent predictors of mortality included male gender,), non-white race, lowest median household income quartile), and having undergone operative intervention. Frail patients waited longer for surgery ( 3.85 days vs 2.44 days; $\mathrm{p}<0.001$ ). Having private insurance was independently associated with earlier operation. Frail patients who waited longer for surgery had higher mortality ().

Conclusion: There is an increasing population of frail older patients with acute small bowel obstruction. Patients with frailty have higher mortality, more frequent discharge to dependent living, longer hospital length of stay, and longer wait to operative intervention. Mortality is also associated with male gender, non-white race, transfer status from another facility, non-private insurance, and low household income. Future study should investigate if outcomes may be improved in the frail geriatric population with earlier operation and sooner return of enteral nutrition.

\section{S095}

\section{Use of Minimally Invasive Surgery for the Management of Hollow} Viscus Traumatic Injuries.

Andrew Litwin, MD; Aixa Perez, MS, MPH; John Romanelli, MD; Michael Tirabassi, MD; Baystate Medical Center.

Introduction: The objective of this study is to assess surgical outcomes following the initial use of laparoscopy versus laparotomy when managing patients with hollow viscus injuries due to trauma.

Methods and Procedures: Using the database from the American College of Surgeons Trauma Quality Improvement Program(TQIP) we identified all patients from 2015-2017 with ICD-10 Diagnosis codes for hollow viscus trauma. Types of procedures were identified by ICD-10 PCS codes. We separated surgical patients into groups based on surgical intervention, mechanism of injury, and type of procedure. Patient outcomes were stratified by major trauma(ISS $>15$ ) and minor trauma. Continuous variables were compared by ANOVA and categorical variables compared by Chi-Square. Analysis performed using STATA 16.

Results: We identified 16,284 patients that matched inclusion criteria within the study time frame. Of those, 1986 patients received a surgical intervention, 1911(96\%) were open and $75(4 \%)$ were laparoscopic. There was no significant difference in demographics. In blunt trauma there were 106 diagnostic procedures, 87(82\%) were open and $19(18 \%)$ were laparoscopic. There were 574 therapeutic procedures, $543(95 \%)$ were open and $31(5 \%)$ were laparoscopic. In penetrating trauma there were 223 diagnostic procedures, $215(96 \%)$ were open and $8(4 \%)$ were laparoscopic. There were 1039 therapeutic procedures for penetrating trauma, 1023(98\%) were open and $16(2 \%)$ were laparoscopic.

For minor trauma, mean length of stay(days) after open surgery was 5 while for laparoscopy it was $2(\mathrm{P}=0.04)$. There were 203 complications noted in the open group and 7 in the laparoscopic group $(\mathrm{P}=0.19)$. For discharge disposition in the open group there were $1026(89 \%)$ home, $111(9.6 \%)$ to a facility, and $16(1.4 \%)$ deaths. In the laparoscopic group 58(93.5\%) home, $3(4.8 \%)$ to a facility, and $1(1.6 \%)$ death $(\mathrm{P}=0.45)$

For major trauma, mean length of stay(days) after open surgery was 5 while for laparoscopy it was $6(\mathrm{P}=0.54)$. There were 242 complications noted in the open group and 1 noted in the laparoscopic group $(\mathrm{P}=0.07)$. For discharge disposition in the open group there were $487(67 \%)$ home, $162(22 \%)$ to a facility, and $76(10 \%)$ deaths. In the laparoscopic group $10(83.3 \%)$ home, $1(8.3 \%)$ to a facility, and $1(8.3 \%)$ death $(\mathrm{P}=0.46)$.

Conclusion: For those that required surgery for hollow viscus injury, laparoscopy appears to be safe, with decreased hospital stay in patients with minor trauma and a trend toward increased likelihood of discharge to home for major and minor trauma. Importantly, this option appears to be underutilized despite these potential benefits, particularly when only a diagnostic procedure was required. 
S096

\section{Is New York State Good at Managing Hollow Vicus Injury?}

Iman Simmonds, MD, MPH; Lorin Towle-Miller; Ajay Myneni, MBBS, PhD; Justin Gray, MD; Anne Stoklosa; Aaron Hoffman, MD; Jeffrey M Jordan, MD, PhD; Ekatarina Noyes, $\mathrm{PhD}$, MPH; University at Buffalo.

INTRODUCTION: Nearly 2 million Americans are admitted to hospital due to trauma annually, with abdominal trauma being second most frequent cause of trauma mortality. While the guidelines for managing penetrating trauma are widely accepted, diagnosis and management of blunt abdominal trauma remains challenging. This study examines current patterns of surgical management of hollow viscus injury (HVI) to assess the long-term patient outcomes and identify areas for quality improvement.

METHODS: Using data from New York Statewide Planning and Research Cooperative System, we identified patients admitted with abdominal injury between 2006-2014. We dichotomized patients by mechanism of injury trauma based on the External Cause of Injury Codes into blunt or penetrating trauma. We selected individuals requiring surgical exploration, primary repair, or stoma using ICD9 procedure codes. We examined process measures, 30-day outcomes and proportion of patients with stoma revision, adjusting for the effect of race, sex, gender, comorbidity, injury severity score, head trauma, transfer status and insurance status at time of injury.

RESULTS: We identified 13,146 hospitalized patients with abdominal injury, 42\% of them $(n=4857)$ had HVI. Penetrating trauma were the most likely cause of HVI $(58 \%)$. Nearly $70 \%$ these patients were managed at a Level 1 trauma center. Eightysix percent of HVI patients were taken to the OR within $24 \mathrm{~h}$; delays were associated with higher rates of diversion, but not mortality. Overall crude mortality for blunt HVI was $4.38 \%$ compared to $8.23 \%$ for penetrating HVI $(\mathrm{p}=0.0016)$. The rates of serious complications were less than $10 \%$ with the exception of development of intrabdominal abscess $(16 \%)$. The colon was the second most commonly injured HVI, regardless of mechanism $(67 \%)$, with $75 \%(n=1,354)$ of colonic injuries repaired primarily. PR patients experienced a shorter length of stay, fewer complications, and higher rates of discharge home without assistance than those managed with diversion $(\mathrm{p}<0.05)$. Only $50 \%$ of stoma patients were reversed by 4 years. CONCLUSIONS: The majority of abdominal trauma in NYS was caused by motor vehicle accidents, falls, and violent assault. Colon injury was the second most common HVI among both blunt and penetrating injuries. Most patients with abdominal trauma were funneled to the appropriate level of care and received operative intervention within $24 \mathrm{~h}$ of initial presentation, and the majority of colonic injuries were managed with primary anastomosis. Despite the excellent short-term quality indexes, stoma reversal rates remain low. More research is needed to better capture and improve long-term consequences of abdominal trauma.

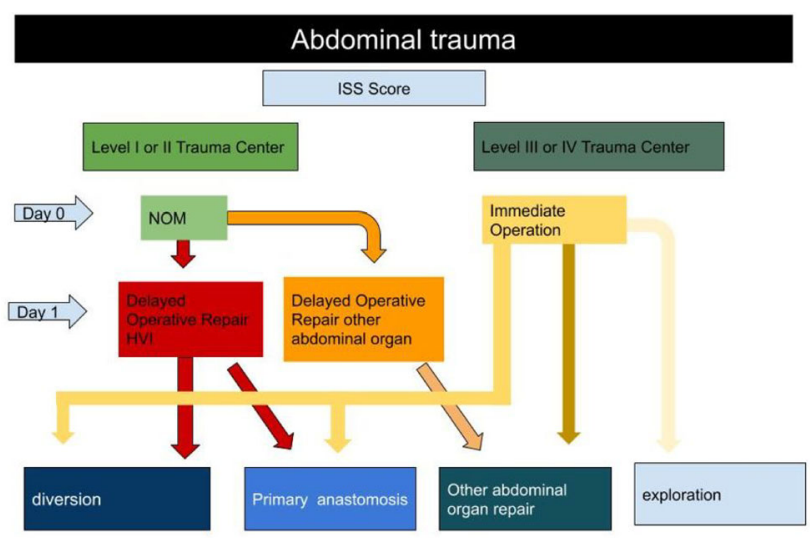

S097

Primary Peritoneal Mesothelioma: A Population-Based Clinical Outcomes Study Involving 1,998 Patients from the Surveillance Epidemiology and End Result (SEER) Database (1975-2016)

Abdul Waheed $^{1}$; Wazir Akbar, MBBS, FCPS ${ }^{2}$; Shaikh A Hai, MD, FACS ${ }^{3}$; Friedrick D Cason, MD, FACS ${ }^{4}$; Ji Fan, MD ${ }^{5}$; Veronica Tucci, MD ${ }^{6}$; John R Monson, MD, FACS, FASCRS ${ }^{7}$; Bruce Orkin, MD, FACS, FASCRS ${ }^{7}$; Matthew Albert, MD, FACS, FASCRS ${ }^{7}$; Noor Nama, MBBS ${ }^{2}$; Subhasis Misra, MD, FACS ${ }^{5} ;{ }^{1}$ University of South Florida; ${ }^{2}$ Bolan Medical College Quetta, Pakistan; ${ }^{3}$ East Florida Division GME Consortium, Westside Regional Medical Center, Plantation, FL, USA; ${ }^{4}$ San Joaquin General Hospital, French Camp, California, USA; ${ }^{5}$ Brandon Regional Hospital, Brandon, Florida, USA; ${ }^{6}$ Oakhill Hospital, Florida, USA; ${ }^{7}$ Center for Colon and Rectal Surgery, AdventHealth Central Florida Division.

Introduction: Primary peritoneal mesothelioma (PPM), first described in 1908 by Miller and Wynn, is a rare, aggressive, and lethal tumor which may arise from both the visceral and parietal peritoneum. Diagnosis and treatment of PPM are often delayed because of their non-specific and highly variable clinical presentations, which may mimic neoplasms originating from other abdominal organs. Patients with PPM have an abysmal prognosis with a median survival of 6-12 months. The current study investigates the demographic, clinical, and pathological factors impacting patients' prognosis and survival with PPM.

Methods: Demographic and clinical data of 1,998 patients with PPM was abstracted from the Surveillance Epidemiology and End Result (SEER) database (1975 - 2016). Chi-square test, paired t-test, multivariate analysis was used to analyze the data.

Results: Patients with PPM were more likely to be male $(56.2 \%, \mathrm{p}<0.005)$ and Caucasian $(90.4 \%, \mathrm{p}<0.005)$. The mean age of diagnosis was $69 \pm 13$ years. Grading information and histological information revealed that poorly differentiated tumors (Grade 3) (49.6\%) and epithelioid histological type $(84.2 \% \%)$ were most common, $\mathrm{p}=<0.005$. Most of PPM were $>4 \mathrm{~cm} \mathrm{~cm}$ in size $(75.1 \% \%)$ and had metastatic at presentation $(53.3 \% \%), \mathrm{p}=<0.001$. For those patients who were treated, the most frequent treatment was chemotherapy (50.6\%). In comparison, sitespecific surgical resection $(29.2 \%)$ and radiation $(1.5 \%)$ were less commonly used, $p$ $=<0.001$ The 5 -year overall survival for PPM was $20.3 \% \pm 1.1$, while those who received surgery, radiation, and chemotherapy had 5-year survivals of $43 \% \pm 5.9$, $25.9 \% \pm 8.4$, and $18.7 \% \pm 1.6$, respectively, $\mathrm{P}=0.037$. Multivariate analysis identified the poor differentiation (odds ratio $(\mathrm{OR})=4.2$, confidence interval $(\mathrm{CI})=$ $3.3-4.9)$, tumor size $>4 \mathrm{~cm},(\mathrm{OR}=3.9, \mathrm{CI}=3.2-4.5)$, epithelioid histological variety $(\mathrm{OR} 4.7, \mathrm{CI}=2.8-6.7)$, Caucasian race $(\mathrm{OR}=2.9, \mathrm{CI}=2.6-4.4)$, and distant SEER stage $(\mathrm{OR}=2.5, \mathrm{CI}=1.1-3.2)$ as independently-associated with increased mortality, $\mathrm{p}<0.001$

Conclusion: PPM is a rare, aggressive tumor of the peritoneum, often difficult to diagnose based on clinical presentation alone. Surgery and chemotherapy are the primary treatment option, while radiotherapy seems to have a limited role. To better understand the pathogenesis and factors impacting survival and improve treatment, we recommend that all patients diagnosed with PPM be enrolled in a nationwide registry. 
S098

\section{Automatic Hierarchical Task Segmentation of Laparoscopic Cholecystectomy Videos Using Deep Learning}

Babak Namazi, PhD ${ }^{1}$; Niyenth K Iyengar ${ }^{2}$; Steven G Leeds, MD $^{1}$; James W Fleshman, $\mathrm{MD}^{1}$; Venkat Devarajan, $\mathrm{PhD}^{3}$; Ganesh Sankaranarayanan, $\mathrm{PhD}^{1} ;{ }^{1}$ Baylor University Medical Center; ${ }^{2}$ University of Texas at Austin; ${ }^{3}$ University of Texas at Arlington.

Introduction: Analyzing surgical workflow is a critical step in surgical video analysis. Hierarchical Task Analysis (HTA) is a well-known method that breaks down any given procedure into phases, tasks, subtasks, and up to motion level descriptors and has been successfully used in various minimally invasive procedures. Recently, deep learning models have been adopted to help with automated surgical workflow analysis, but their application has been limited to identification of surgical phases and tools. One of the major bottlenecks to this approach is the requirement of separate models to identify the tasks and subtasks, which can quickly add up to a larger number of models for training. Our objective is to automatically identify phases, tasks, and subtasks in a laparoscopic cholecystectomy (LC) video using a deep multi-task learning.

Methods: To perform hierarchical segmentation, each frame of a LC video was manually annotated for the start and end of each of the four phases, eight tasks, and nineteen subtasks. We adopted Resnet50, a deep Convolutional Neural Network (CNN) for our multi-task model to perform frame-level visual feature extraction and classification. We also trained three individual models for phases, tasks, and subtasks detection for comparison of performance.

Results: We used the publicly available cholec 80 dataset, which contains 80 videos of LC surgeries performed by 13 surgeons. All the frames were extracted ( 30 frames per second) and annotated. The training set contained 40 videos, and the other 40 videos were used for validating the performance of the model. The training set was balanced to have an equal number of frames for each subtask from each training video. The accuracy and per-class F1-score for both models are shown in Table 1 . Conclusion: We obtained improved performance by using a multi-task training model for identifying phases, tasks and subtasks compared to the individual model training. This is due to the exploitation of the correlation among the patterns and the hierarchical structure of the problem. Furthermore, the inference on the joint model was three times faster and required less computation and memory usage. Further improvement in the performance of our multi-task model using temporal features will be our future work

Table 1 Comparison of the Performance for our Multi-Task Learning for Hierarchical Task Segmentation of LC Videos

\begin{tabular}{|c|c|c|c|c|}
\hline \multirow{2}{*}{ Categories } & \multicolumn{2}{|c|}{$\begin{array}{c}\text { Single Task } \\
\text { Training }\end{array}$} & \multicolumn{2}{c|}{$\begin{array}{c}\text { Multi-Task } \\
\text { Training }\end{array}$} \\
\cline { 2 - 5 } & $\begin{array}{c}\text { Accuracy } \\
(\%)\end{array}$ & $\begin{array}{c}\text { F1- } \\
\text { Score } \\
(\mathbf{\%})\end{array}$ & $\begin{array}{c}\text { Accuracy } \\
\mathbf{( \% )}\end{array}$ & $\begin{array}{c}\text { F1- } \\
\text { Score } \\
\mathbf{( \% )}\end{array}$ \\
\hline Phases & 78.48 & 69.71 & 82.18 & 73.02 \\
\hline Tasks & 68.68 & 52.93 & 70.62 & 56.09 \\
\hline Subtasks & 56.93 & 35.39 & 58.82 & 37.68 \\
\hline
\end{tabular}

S099

\section{ATTENDING GUIDANCE ADVISED: EDUCATIONAL QUALITY OF SURGICAL VIDEOS ON YOUTUBE}

Hope T Jackson, MD, FACS; Chen-min S Hung, BA; Deepika Potarazu, BS; Noor Habboosh, BA; Erik J DeAngelis, MD; Richard L Amdur, PhD; Jordan M Estroff, MD, FACS; Megan T Quintana, MD; Paul Lin, MD, FACS; Khashayar Vaziri, MD, FACS; Juliet Lee, MD, FACS; Department of Surgery, George Washington University.

Introduction: YouTube is the most used platform for case preparation by surgical trainees. Despite its popular use, studies have noted limitations in technique, safety, and vetting of these videos. This study identified the most viewed laparoscopic cholecystectomy (LC) videos on YouTube and analyzed the ability of faculty, residents, and medical students to identify critical portions of the procedure, technique, and limitations of the videos.

Methods: An incognito search was conducted on YouTube using the term "laparoscopic cholecystectomy." Results were screened for length, publication date, and language. The top ten videos were presented to general surgery attendings, residents, and medical students at a single institution. Established rubrics were used for evaluation, including the Critical View of Safety (CVS) for LC, a modified Global Operative Assessment of Laparoscopic Skills (GOALS) score, a task-specific checklist, and visual analog scales for case difficulty and operator competence. Educational quality and likelihood of video recommendation for case preparation were evaluated via Likert scales. Attending assessments were considered the gold standard.

Results: Six attending surgeons achieved excellent internal consistency on CVS, educational quality, and likelihood of recommendation scales, with Cronbach alpha (Ca) of $0.93,0.92$, and 0.92 , respectively. Ca was $\geq 0.70$ in all the other scales measured. Only one of the ten videos attained all three established CVS criteria. Four videos demonstrated none of the CVS criteria. The mean educational quality (mEQ) was 4.63 on a 10 -point scale. The mean likelihood of recommendation ( $\mathrm{mLoR}$ ) for case preparation was 2.30 on a 5-point scale. Senior resident assessments (Postgraduate Year $(\mathrm{PGY}) 4+, \mathrm{N}=12$ ) aligned with attending surgeons, with no significant differences in CVS attainment, $\mathrm{mEQ}$, and mLoR. Junior residents (PGY1$3, N=17$ ) and medical students (MS3-4, N = 20) exhibited significant difference with attendings in CVS attainment, $\mathrm{mEQ}$, and $\mathrm{mLoR}$ for more than half the videos. Both groups tended to overrate videos compared to attendings.

Conclusion: To our knowledge, this is the first study to examine faculty, resident, and student assessments of surgical videos on a social media platform. The most viewed LC videos on YouTube did not attain the CVS, had low educational value, and were inappropriate for case preparation. Senior resident video assessments closely align with attendings, while junior trainees are more likely to overstate video quality and value. Faculty guidance and direction of trainees to high-quality, vetted resources for surgical case preparation is needed, and surgical societies should prioritize creation and dissemination of high-quality videos on public platforms. 


\section{S100}

Correlation of novice's performance to various objective stress parameters during basic skills training in a high-fidelity simulator.

Konstantinos Georgiou, MD, MSc, $\mathrm{PhD}^{1}$; Nikola Boyanov, $\mathrm{MD}^{2}$; Alexander Chamzin, $\mathrm{MD}^{1}$; Dimitrios Thanasas, $\mathrm{PhD}^{3}$; Gabriel Sandblom ${ }^{4}$; Spyridon Stergiopoulos, $\mathrm{MD}^{1}$; Blagoi Marinov, $\mathrm{MD}, \mathrm{PhD}^{2}$; Lars Enochsson, $\mathrm{MD}^{5} ;{ }^{1} 1 \mathrm{st}$ Department of Propaedeutic Surgery, Hippokration General Hospital of Athens, Athens Medical School, National and Kapodistrian University of Athens, Athens, Greece; ${ }^{2}$ Medical Simulation Training Center, Medical University of Plovdiv, Bulgaria; ${ }^{3}$ Medical Physics Laboratory Simulation Center, Athens Medical School, National and Kapodistrian University of Athens, Athens, Greece; ${ }^{4}$ Department of Clinical Science and Education Södersjukhuset, Karolinska Institutet, Stockholm, Department of Surgery, Södersjukhuset, Stockholm, Sweden; ${ }^{5}$ Division of Surgery, Department of Surgical and Perioperative Sciences, Umeå University, Umeå, Sweden.

Introduction: Several non-invasive stress measuring methods have been proposed to measure surgeon's stress in the OR. Little evidence exists on which parameter better represents how much stress a novice experiences during VR simulation training. This study concomitantly measures responses of all previously reported objective non-invasive stress indices. Compares them to performance as measured by video score (VS) achieved by novice surgeons in a reproducibly stressful simulation environment.

Methods: 65 male PGY1 + 2 laparoscopy naive trainees enrolled. After orientation phase, wore a wrist device (E4WB) measuring heart rate (HR), interbeat interval (IBI) duration, electrodermal activity (EDA). Saliva specimen collected for alphaamylase (sAA), cortisol (sC), secretory immunoglobulin A ( $\operatorname{sIg} \mathrm{A})$ and Chromogranin $\mathrm{A}(\mathrm{sCgA})$ measurements (baseline phase, $\mathrm{BL})$. Then, the simulation exercise phase (E) started. Subjects trained on basic skills module. Immediately after, another saliva sample collected for estimating biochemical markers. Whole experiment was videotaped. VS calculated by two reviewers blinded to identity and results. Percentage (E-BL) diff of each of 7 parameters was calculated and compared with VS using Pearson's correlation coefficient and Akaike Information Criterion (AICc) (Table 1).

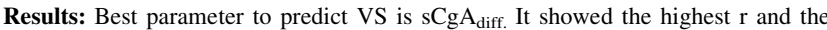
lowest $\mathrm{AICc}$ value, followed by $\mathrm{EDA}_{\text {diff, }}$ and $\mathrm{sAA}_{\text {diff. }} \mathrm{sCgA}$ had higher average percentage increase, followed by EDA.

Conclusions: In our simulation setting, sympathetic ANS stress related saliva biomarkers could accurately describe novice trainees' performance measured by VS. Those easy to assess non-invasive indices can be implemented to estimate stress levels during training simulation process.

Table 1: Mean \pm SD, Range of parameter, Pearson correlation coefficient (r) with VS and corrected AICc value

\begin{tabular}{lclcc}
\hline Parameter & Mean \pm SD & Range $(\mathbf{m i n}, \mathbf{m a x})$ & $\mathbf{r}(\mathbf{p}>\mathbf{0 . 0 1})$ & AICc \\
\hline $\mathbf{S A A _ { \text { diff } }}$ & $120 \pm 98$ & $(5,377)$ & 0.61 & 246.2 \\
$\mathbf{S C}$ & $54 \pm 72$ & $(1,263)$ & 0.46 & 260.34 \\
$\mathrm{SIgA}_{\text {diff }}$ & $162 \pm 157$ & $(3,516)$ & 0.52 & 255.49 \\
$\mathrm{sCgA}_{\text {diff }}$ & $2451 \pm 854$ & $(1013,4165)$ & 0.71 & 231.02 \\
$\mathrm{EDA}_{\text {diff }}$ & $910 \pm 354$ & $(386,1652)$ & -0.64 & 242.28 \\
$\mathrm{IBI}_{\text {diff }}$ & $1.3 \pm 5.5$ & $(-6.9,14.9)$ & 0.43 & 262.93 \\
$\mathrm{HR}_{\text {diff }}$ & $-1.0 \pm 5.7$ & $(-13.1,8.2)$ & -0.40 & 264.65 \\
\hline
\end{tabular}

\section{S101}

Preliminary Findings in the Transfer of Virtual Reality Endoscopy Training to Live-Animal Colonoscopy: A Randomized Control Trial of Proficiency vs. Repetition Based Training

Douglas J Cassidy, $\mathrm{MD}^{1}$; Taylor M Coe, $\mathrm{MD}^{1}$; Kristen M Jogerst, MD, MPH ${ }^{2}$; Yoon Soo Park, PhD ${ }^{1}$; Sophia K McKinley, MD, EdM ${ }^{1}$; Naomi M Sell, MD ${ }^{1}$; Emil Petrusa, $\mathrm{PhD}^{1}$; Daniel A Hashimoto, MD, MS ${ }^{1}$; Robert N Goldstone, $\mathrm{MD}^{1}$; Denise W Gee, MD ${ }^{1} ;{ }^{1}$ Massachusetts General Hospital; ${ }^{2}$ Mayo Clinic Arizona.

Introduction: Low first-time pass rates of the Fundamentals of Endoscopic Surgery (FES) have stimulated development of virtual reality (VR) simulation curricula for resident preparation. The purpose of this study is to evaluate the transfer of VR endoscopy training to live porcine colonoscopy and endoscopy.

Methods: 24 novice endoscopists, general surgery interns with less than 10 clinical endoscopies, at a single tertiary academic center completed pretesting via the FES manual skills examination and Global Assessment of GI Endoscopic Skills (GAGES) assessment of porcine upper and lower endoscopy. Participants were then randomly assigned one of two GI Mentor VR curricula: proficiency-based ( 2 consecutive trials of each task at or above expert-derived performance standards) or repetition-based (10 repetitions of each task) training. Following completion of the assigned curriculum, participants completed post testing via the FES manual skills examination and GAGES assessment of porcine upper and lower endoscopy by blinded experts. Post-testing is underway however the COVID-19 pandemic has delayed completion. Group demographics were compared with t-test, Wilcoxon Rank Sum, and Chi-square to ensure balanced randomization. Emerging GAGES data is reported using descriptive statistics and compared with an ANCOVA regression model.

Results: There were no baseline demographic differences between the repetition and proficiency groups in gender, age, glove size, prior GI mentor experience, or mean number of prior clinical endoscopies or colonoscopies performed. Mean baseline performance on FES manual skills pre-testing (proficiency $=48.2$, repetition $=47.9$; $\mathrm{p}=0.96$ ) and composite porcine endoscopy GAGES scores (proficiency $=20.8$, repetition $=20.7 ; p=0.93$ ) were comparable between the two groups. The groups performed similarly on both the porcine upper endoscopy (proficiency $=9.7$, repetition $=10.9 ; \mathrm{p}=0.33$ ) and colonoscopy (proficiency $=11.1$, repetition $=9.7 ; \mathrm{p}=$ 0.22 ). Eleven residents completed the porcine endoscopy and colonoscopy posttesting. There was a significant improvement in post-curricular porcine endoscopy performance as measured by mean GAGES scores (upper endoscopy: 5.8 (SD 4.7) p $=0.002$; colonoscopy: 6.4 (SD 4.2), $\mathrm{p}<0.001)$. The post-test results did not differ between the proficiency and repetition curricular intervention $(\mathrm{F}=0.84, \mathrm{p}=0.47)$. Conclusion: There was no difference amongst trainees randomized to the repetition and proficiency curricula. Preliminary results from the post-testing demonstrate transfer of endoscopic skills from VR curricula to live endoscopy. However, the improvement of endoscopic skills was not significant when adjusting for type of curriculum completed. Remaining post-testing will be completed within the next six months, allowing full analysis of the transfer of VR endoscopic skills to a live animal model as well as further comparison of proficiency vs. repetition-based VR training. 


\section{$\mathbf{S 1 0 2}$}

Safer Operating Room Teams: Rationale for the Fundamental Use of Surgical Energy (FUSE) Hospital Compliance Module

Jaisa Olasky, MD ${ }^{1}$; Edward L Jones, $\mathrm{MD}^{2}$; Daniel B Jones, MD, $\mathrm{MS}^{3}$; Thomas N Robinson, MD, MS ${ }^{2} ;{ }^{1}$ Mount Auburn Hospital; ${ }^{2}$ Rocky Mountain Regional VA Medical Center; ${ }^{3}$ Beth Israel Deaconess Medical Center.

Introduction: The Fundamental Use of Surgical Energy (FUSE) program was developed by The Society of American Gastrointestinal and Endoscopic Surgeons (SAGES) to promote the safe use of surgical energy. A curriculum that could be used in hospital educational programs was needed to expand access. The goal of this project was to develop a short, inexpensive, online module that emphasizes key FUSE learning objectives (Fig. 1). The accompanying survey (Fig. 2) assessed perceived relevancy.

Methods and Procedures: The SAGES FUSE Committee developed the Hospital Compliance Module. The target audience included all OR personnel. The Module was piloted at Beth Israel Deaconess Medical Center. The data was analyzed using Chi-square with Yates' correction two-tailed test

Results: Three-hundred-eighty individuals completed the survey: 198(52\%) surgeons, $139(37 \%)$ nurses, $28(7 \%)$ surgical technicians and $15(4 \%)$ housestaff. See Table 1 for responses by learner role.

For "...the Module taught me valuable information" $155(41 \%)$ responded extremely and $350(92 \%)$ responded at least somewhat.

For "As a result of [the Module] how likely are you to change how you set up or use energy devices...?" $103(27 \%)$ responded extremely and $305(80 \%)$ responded at least somewhat.

For "How likely are you to recommend this compliance module...?" 143(38\%) responded extremely and $333(88 \%)$ responded at least somewhat.

Conclusions: The FUSE Hospital Compliance Module is effective and efficient. It should be considered for widespread distribution by hospitals to enhance staff education.

Figure 1. FUSE HOSPITAL COMPLIANCE MODULE CONTENT BLUEPRINT

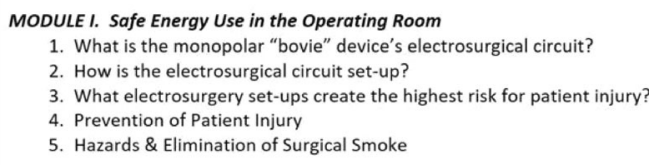

3. What are the preferred energy-based devices to use in a patient with a cardiac implanted device?

4. Important clinical concepts regarding surgical energy and implanted cardiac devices

Four Multiple Choice Questions
TABLE 1. Comparison of Responses Between Surgeons and Nurses

\begin{tabular}{|c|c|c|c|}
\hline & Surgeons & Nurses & p-value \\
\hline \multicolumn{4}{|c|}{ I believe that the FUSE Compliance Module taught me valuable information } \\
\hline Extremely & $69(35 \%)$ & $71(51 \%)$ & $\mathrm{p}=0.004$ \\
\hline Extremely/Moderately & $130(66 \%)$ & $123(88 \%)$ & $\mathrm{p}<0.001$ \\
\hline Extremely/Moderately/Somewhat & $173(87 \%)$ & $136(98 \%)$ & $\mathrm{p}=0.001$ \\
\hline \multicolumn{4}{|c|}{$\begin{array}{l}\text { As a result of learning this material how likely are you to change how you set up or } \\
\text { use energy devices in the operating room? }\end{array}$} \\
\hline Extremely & $46(23 \%)$ & $45(32 \%)$ & $\mathrm{p}=0.083$ \\
\hline Extremely/Moderately & $106(54 \%)$ & $99(71 \%)$ & $\mathrm{p}=0.002$ \\
\hline Extremely/Moderately/Somewhat & $147(74 \%)$ & $123(88 \%)$ & $\mathrm{p}=0.002$ \\
\hline \multicolumn{4}{|c|}{ How likely are you to recommend this compliance module to your colleagues? } \\
\hline Extremely & $61(31 \%)$ & $65(47 \%)$ & $\mathrm{p}=0.004$ \\
\hline Extremely/Moderately & $121(61 \%)$ & $110(79 \%)$ & $\mathrm{p}=0.001$ \\
\hline xtremely/Moderately/Somewhat & $163(82 \%)$ & $131(94 \%)$ & $\mathrm{p}=0.002$ \\
\hline
\end{tabular}

Figure 2. FUSE HOSPITAL COMPLIANCE MODULE SURVEY

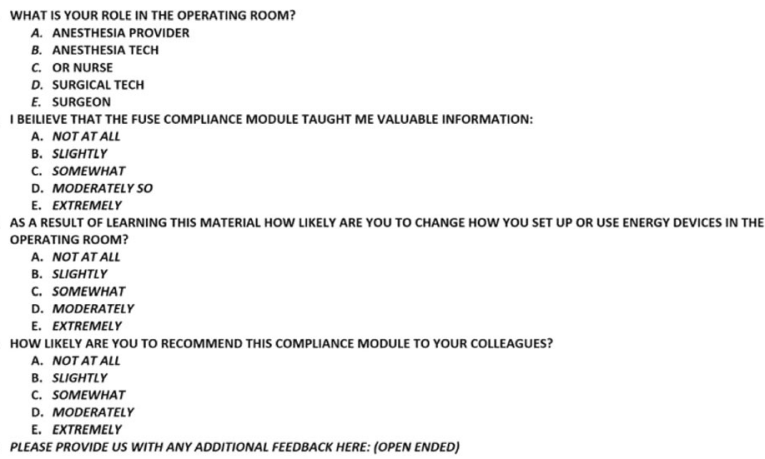




\section{$\mathbf{S 1 0 3}$}

\section{SURVEY OF THE 2020 FELLOWSHIP COUNCIL APPLICATION AND MATCH PROCESS AND THE IMPACT OF COVID-19}

Amy L Rosenbluth, $\mathrm{MD}^{1}$; Madhuri B Naharaj, MD, MS${ }^{2}$; Michael Brunt, $\mathrm{MD}^{3}$; Daniel J Scott, $\mathrm{MD}^{2} ;{ }^{1}$ Stony Brook; ${ }^{2} \mathrm{UT}$ Southwestern; ${ }^{3}$ Washington University School of Medicine.

Introduction: The interview process represents a necessary but resource intensive process from applicant and program perspectives. This study aimed to identify opinions of the 2020 Fellowship Council (FC) application and match process and incycle transition to virtual interviews due to the COVID-19 pandemic.

Methods: Questionnaires were developed to assess aspects of the interview and match process including: number of interviews, cost, time burden, unplanned changes in format, and effectiveness. These were distributed by the FC to all applicants and fellowship programs following the match process. Completion was voluntary and data (reported as median[IQR]) were anonymous.

Results: Applicant response rate was 53\% (140/265 certified applicants). Applicants submitted 27.5 (13.25-40) applications to 2(2-3) types of program accreditations, were offered interviews at $10(4-17)$, and ranked 10(5-15) programs. Due to COVID$19,74 \%$ of interview plans changed and applicants completed only $30 \%$ of their planned in-person interviews. For decision-making, $90 \%$ felt that in-person and $81 \%$ virtual interviews were helpful. Total expected cost was $\$ 4,750(\$ 2,000-\$ 6,000)$ vs actual cost $\$ 1,000(\$ 250-\$ 2,250)(\mathrm{P}-\mathrm{value}<0.05)$. Total expected missed work-days were 10(5-16) vs actual 3(0-6.25) (P-value $<0.05)$.

Program response rate was 38\%(55/143). Programs received 60(43-85.5) applications, offered 20(15-26) interviews, completed 16(12.5-21) interviews, and ranked 14(10-18) candidates. For decision-making, $92 \%$ felt in-person vs $71 \%$ virtual interviews were helpful. Expected in-person resources were 2.5(2-3.25) days with 4(3-5) faculty spending 5(5-7) hours per day. When only virtual resources were used, median number of sessions were $2(1-3)$, with $3(2.5-4)$ faculty spending $4(3-5)$ hours per day. When comparing person-hours(days*faculty*hours), there was a statistical difference between expected in-person 48(27.5-80) and virtual 24(9-40)(P-value $<0.05)$ interviews.

\begin{tabular}{llllll}
\hline Future Interview Format Preferences & & & \\
\hline & $\begin{array}{l}\text { In-Per- } \\
\text { son, } \\
\text { site }\end{array}$ & $\begin{array}{l}\text { In-Person, } \\
\text { Centralized }\end{array}$ & $\begin{array}{l}\text { In-Person, On Site } \\
\text { or Centralized }\end{array}$ & $\begin{array}{l}\text { In-Person After Vir- } \\
\text { tual Pre-Interview }\end{array}$ & $\begin{array}{l}\text { Virtual } \\
\text { Only }\end{array}$ \\
\hline Applicants & $18 \%$ & $1 \%$ & $8 \%$ & $44 \%$ & $29 \%$ \\
Programs & $31 \%$ & $2 \%$ & $9 \%$ & $38 \%$ & $21 \%$
\end{tabular}

Conclusion: Despite format changes, $81 \%$ of applicants and $71 \%$ of programs felt they gained sufficient information from virtual sessions to create their rank lists. Virtual interviews were associated with decreased cost and fewer missed work-days for applicants and decreased resource usage for programs. While applicants and programs felt inperson interviews were more helpful, the majority of both groups preferred either a solely virtual or a virtual pre-interview format. Given these findings, virtual interviews should be incorporated into future fellowship application cycles beyond the pandemic

\section{$\mathbf{S 1 0 4}$}

Robotic Surgery Training Curricula: Prevalence, Perceptions, and Educational Experiences in General Surgery Residency Programs

Matthew P Madion, MD; Andrew Kastenmeier, MD; Matthew I Goldblatt, MD; Rana M Higgins, MD; Medical College of Wisconsin.

Purpose: Utilization of robotics in general surgery has increased exponentially in the past several years. However, there are no national training standards or formal case requirements for residency training. The purpose of this study was to provide an updated analysis of the prevalence of robotic training curricula among general surgery residency programs across the United States.

Methods: A 19-item electronic survey was constructed and distributed to program directors of the Association of Program Directors in Surgery listserv. The survey focused on the residency programs' demographics, program directors' opinions of robotic surgery, and status of robotic surgery training curricula. Data was collected, compiled and analyzed using Qualtrics Survey Software, Microsoft Excel and IBM SPSS. Chi-Squared statistical significance was defined as a p-value of $<0.05$.

Results: Of the 280 program directors, 107 (38.2\%) responses were received. Overall, $75(70 \%)$ residency programs provided a formal robotic surgery curriculum (Table 1). Regarding the importance of robotics to general surgery training, 67 $(89 \%)$ programs that provided a formal robotic surgery curriculum stated it was either 'Very important' or 'Probably important' as opposed to $23(72 \%)$ programs that did not offer a formal robotic surgery curriculum $(\mathrm{p}=0.017)$ (Table 1). Of the residency programs with a formal robotic surgery training curriculum, $58(79 \%)$ had been present for 3 years or less. Bedside assisting began in $62(85 \%)$ programs as a PGY1 or PGY2 and residents began operating on the console as a PGY2 or PGY3 in $53(72 \%)$ programs. However, there was variability regarding the percentage of the case a senior resident actually operated on the robotic console (Table 1).

Conclusions: A majority of general surgery residency programs offer formal robotic surgery curricula and have been present for 3 years or less. Most residencies begin their curricula in PGY1 or PGY2 year, with an opportunity to bedside assist and operate on the robotic console in the first three years of residency. However, there is significant variability in the actual percentage of time that senior residents are operating on the robotic console. These findings highlight that more defined operative milestones for robotic general surgery cases need to be identified. Additionally, educators should further investigate barriers preventing residents from operating on the robotic console within their own institutions.

Table 1. Program demographics, program directors' perception, and program details of robotic surgery training.
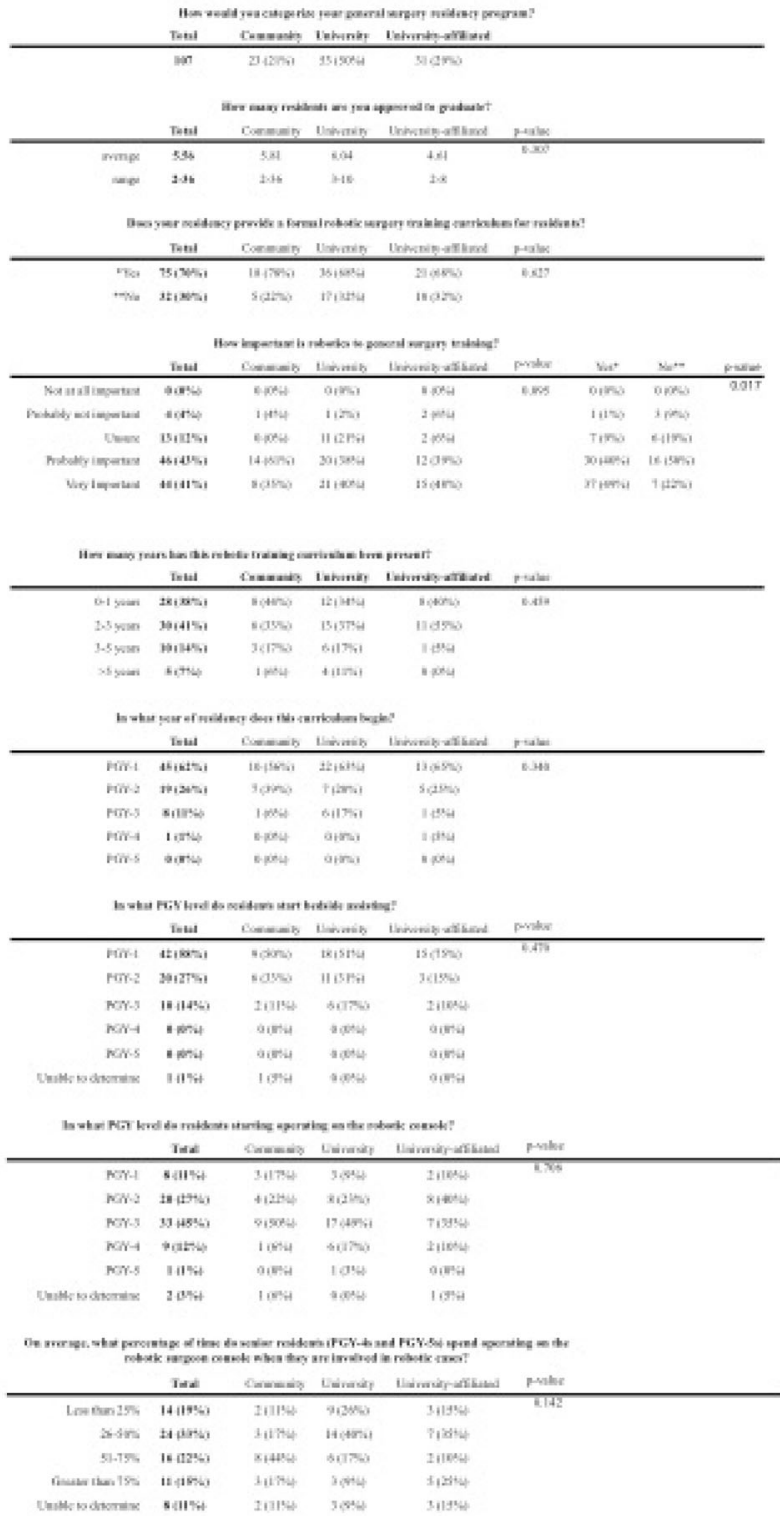
S105

\section{SAFETY AND FEASIBILITY OF SAME-DAY DISCHARGE FOR ELECTIVE MINIMALLY INVASIVE COLECTOMY WITH REMOTE TELEMEDICINE FOLLOW-UP}

Jules H Eustache, $\mathrm{Dr}^{1}$; Motahar Basam, $\mathrm{Dr}^{2}$; Sender A Liberman, $\mathrm{Dr}^{1}$; Andrew G Rudikoff, Dr ${ }^{2}$; Liane $\mathrm{S}$ Feldman, $\mathrm{Dr}^{1}$; Elisabeth C McLemore, $\mathrm{Dr}^{2}$; Lawrence Lee, $\mathrm{MD}, \mathrm{PhD}^{1} ;{ }^{1}$ McGill University Health Centre; 2Kaiser Permanente Los Angeles Medical Center.

BACKGROUND: Enhanced recovery pathways have reduced complications and hospitalization after colorectal surgery, and one next step of this perioperative paradigm is same day discharge. The use of telemedicine and remote patient monitoring may allow for same-day discharge(SDD) after laparoscopic colectomy. Therefore, we sought to investigate the safety and feasibility of same-day discharge after elective laparoscopic colectomy with post-discharge remote monitoring. METHODS: A prospective study was conducted at two university-affiliated centres starting from 01/2020. Adult patients undergoing elective minimally-invasive colectomy(including stoma reversal) were screened for eligibility. Eligibility criteria included ability to comply with telemedicine follow-up, adequate support at home, and living in proximity $(<1 \mathrm{~h})$ to the hospital. Exclusion criteria included significant comorbidities, planned multi-visceral resection, creation of a new stoma, conversion to open, cognitive impairment, and inadequate home support. Patients were discharged from the recovery room if they tolerated clear liquids, had adequate analgesia with oral medications, and were able to ambulate and urinate independently. Patients that failed these criteria were admitted with planned discharge on POD1. All patients had post-discharge monitoring using a mobile application or were contacted by telephone daily(for 7 days). The main feasibility outcome was emergency department(ED) visit within 3 postoperative days.

RESULTS: Of the 56 patients recruited, successful SDD occurred in $67.9 \%$, with $28.6 \%$ discharged on POD1, and $3.6 \%$ on POD2(Table 1). Postoperative nausea/ vomiting were the most common reasons for same-day admission(Table 1). Only one patient failed SDD protocol( $2 \%)$ and required readmission on POD1 due to pain and urinary retention. One patient $(2 \%)$ went to clinic within 3 days for a leaking foley catheter. Within 30 days, 9 patients $(16 \%)$ visited the ED, 7 of which $(13 \%)$ were readmitted. Except for the single patient who returned POD1, all other ED visits occurred on or after POD7. The most common reason for ED visits or readmission at 30 days was C. difficile infection(22\%). There were no reoperations and no deaths. Patients had mean age of 57.3 years(SD12.9), mean BMI of 27.5(SD5.7), and mean Charlson Comorbidity Index of 3.3(SD2.1). Surgical indications included neoplasm $(52 \%)$, stoma closures $(30 \%)$, diverticular disease $(11 \%)$, inflammatory bowel disease $(3.5 \%)$. Subjects underwent left/sigmoid(37.5\%), stoma closures(30\%), right/transverse $(25 \%)$, and rectal resection(7\%). Approach was laparoscopic(70\%), peristomal $(16 \%)$, or robotic(14\%)

CONCLUSION: Our study suggest that same-day discharge with telemedicine post-discharge monitoring is feasible and safe after minimally-invasive colectomy in selected patients.

\begin{tabular}{|c|l|}
\hline Discharge Day & $\%(\mathrm{n})$ \\
\hline POD0 & $67.9 \%(38)$ \\
\hline POD1 & $28.6 \%(16)$ \\
\hline POD2 & $3.5 \%(2)$ \\
\hline Reasons for same-day admission & \\
\hline Nausea/Vomiting & $\%(\mathrm{n})$ \\
\hline Patient refusal & $33 \%(6)$ \\
\hline Urinary issues & $28 \%(5)$ \\
\hline Pain Control & $17 \%(3)$ \\
\hline Bleeding & $11 \%(2)$ \\
\hline Intraoperative complications & $6 \%(1)$ \\
\hline & $6 \%(1)$ \\
\hline
\end{tabular}

\section{S106}

\section{Self-Identified Black Patients Face Reduced Access to Minimally} Invasive Gastrectomies

Sirui Ma, MD ${ }^{1}$; Mario M Matabele, $\mathrm{BS}^{2}$; Kasey L Wood, BS ${ }^{2}$; Syed F Haider, MD ${ }^{1}$, Archana Babu, BA, MS ${ }^{1}$; Aziz Merchant, MD ${ }^{3}$; Ravi J Chokshi, MD, MPH, FACS ${ }^{1} ;{ }^{1}$ Division of Surgical Oncology, Department of Surgery, Rutgers New Jersey Medical School, Newark, NJ; ${ }^{2}$ University of Wisconsin School of Medicine and Public Health, Madison, WI; ${ }^{3}$ Division of General Surgery, Department of Surgery, Rutgers New Jersey Medical School, Newark, NJ.

Background: Minimally invasive gastrectomies (MIG), either laparoscopic or robotic, have been demonstrated to have shorter length of stays, fewer complications, greater lymph node yields, comparable negative margins and mortality rates when compared to open total or subtotal gastrectomies. This study aims to identify the racial disparities in the different approaches to a gastrectomy. We further evaluate the relationship between the race and postoperative complications and test the mediating effect of the selected surgical approach.

Methods: This IRB-approved retrospective study utilized data from the American College of Surgeons National Quality Improvement Program. All recorded cases of MIG, open total gastrectomy, and open subtotal gastrectomy between 2012 and 2017 were isolated. Propensity score matching with a 3:1 ratio was conducted to assess the independent effect of black self-identified race on access to MIG. $p<0.05$ was required to achieve statistical significance.

Results: There were 10,203 cases of MIG and open total or subtotal gastrectomy. Of this cohort, 8,379 self-identified as white and 1,824 self-identified as black. Following propensity score matching, there were 5,472 patients of white self-identified race and 1,824 patients of black self-identified race. Analysis of the balanced cohort revealed that black self-identified race was independently associated with significantly reduced access to MIG (43.2\% of white patients versus $34.8 \%$ of black patients, $\mathrm{p}<0.001$ ). No significant association between any of the post-operative complications and length of stay and the black self-identified race.

Conclusion: Our analysis reveals a significant racial disparity in access to MIG. Minimally invasive techniques are underutilized in racial minorities despite their known benefits to patients. However, no significant differences were seen in postoperative complications or length of stay between the racial groups. 
S107

\section{ARTIFICIAL INTELLIGENCE PREDICTION OF CHOLECYSTECTOMY OPERATIVE COURSE FROM AUTOMATED IDENTIFICATION OF GALLBLADDER INFLAMMATION}

Thomas M Ward, MD; Daniel A Hashimoto, MD, MS; Yutong Ban, PhD; Guy Rosman, PhD; Ozanan R Meireles, MD; Massachusetts General Hospital.

Introduction: Operative courses of laparoscopic cholecystectomies vary widely due to differing pathologies. Efforts to assess intra-operative difficulty include the Parkland Grading Scale (PGS), which scores inflammation from 1 to 5 based on the initial view of the gallbladder. We investigated if PGS led to variation of intraoperative outcomes, including operative duration, attainment of the Critical View of Safety (CVS), and gallbladder injury. We additionally trained an Artificial Intelligence (AI) model to identify PGS to allow for real-time prediction of operative events.

Methods: One surgeon labeled timestamps of surgical phases, PGS, CVS attainment, and gallbladder injury in 200 cholecystectomy videos. Each video was linked to the surgeon performing the case with an anonymized identifier. To analyze effect of PGS on overall and per-phase duration, we used a linear regression model and accounted for the effect of different surgeons' technique preferences on the operative course by including a surgeon identifier variable and an interaction variable between surgeon and PGS. To analyze the PGS's effect on CVS attainment and gallbladder injury, we performed logistic regression. We trained an AI model (Convolutional Neural Network) to identify PGS using initial frames from operative videos. To assess the model's predictive reliability, we compared weighted Cohen's kappa between the first surgeon's PGS annotations and both the AI's PGS determination and a second surgeon's annotations.

Results: Each point increase in the PGS was associated with a 7.9-min increase in operative duration $(95 \%$ CI 3.7-12.0, $\mathrm{p}=0.0003)$, 3.0-min increase in active dissection time (95\% CI $0.34-5.6, \mathrm{p}=0.03), 1.4-\mathrm{min}$ increase in time to remove the gallbladder from the liver bed $(95 \%$ CI $0.02-2.2, \mathrm{p}<0.05)$, and a 1.35 increased odds of creating a gallbladder hole (95\% CI $1.03-1.79, \mathrm{p}=0.03)$. PGS did not impact the odds of attaining the CVS $(\mathrm{p}=0.2)$. The AI model was trained to identify PGS on video frames prior to dissection from 144 randomly selected videos. It then determined PGS in the remaining 51 videos. Its reliability did not significantly differ $(\kappa 0.68,95 \%$ CI $0.54-0.79)$ when compared to a second surgeon (к $0.80,95 \%$ CI $0.69-0.87$ ).

Conclusion: AI can reliably identify the PGS from an initial assessment of the gallbladder and use this information to predict the intra-operative course. Automated gallbladder inflammation assessment could optimize operative workflow and generate per-surgeon and per-resident metrics for quality and safety improvements and education.

\section{S108}

\section{THE LEARNING CURVE FOR TAMIS ON USING A SIMULATOR MODEL}

$\underline{\text { Sung Sil Park }}{ }^{1}$; Dae Kyung Sohn ${ }^{1}$; Hongrae Kim ${ }^{2}$; Dong-Eun Lee ${ }^{3}$; Sung Chan Park ${ }^{1}$; Jae Hwan $\mathrm{Oh}^{1} ;{ }^{1}$ Center for Colorectal Cancer, Research Institute and Hospital, National Cancer Center, Goyang, Korea; ${ }^{2}$ Division of Convergence Technology,Research Institute and Hospital, National Cancer Center, Goyang, Korea; ${ }^{3}$ Biostatistics Collaboration Team, Research Institute and Hospital, National Cancer Center, Goyang, Korea.

INTRODUCTION: Transanal minimally invasive surgery (TAMIS) has been widely used as an advanced endoscopic technique for local excision of rectal tumors. However, TAMIS is a technically complex procedure and requires a well-designed training program. We developed a TAMIS simulator model to educate trainees and analyzed the learning curve for TAMIS using this model.

METHODS AND PROCEDURES: The TAMIS simulator model was developed by remodeling an existing laparoscopic training system (Simsei Laparoscopic Trainer) to perform transanal procedures through a single port (GelPOINT® Path) (Fig. 1). From March 2020 to June 2020, 12 trainees (four senior surgical residents and eight colorectal surgical fellows) with an experience in laparoscopic surgery, but not in TAMIS, performed 20 TAMIS training sets each, using the simulator model. All surgical procedures were recorded on video and reviewed. The total operation time consisting of specimen removal and wound closure was recorded. We also graded each of the following four items (a high score indicates good results): the quality of wound closure (1-4), specimen quality (1-4), self-confidence of trainees (1-5), and the evaluation of technical performance by the supervisor (1-5). Using a moving average, we analyzed the number of cases required to reach stabilization of the procedure time. Cumulative sum (CUSUM) analysis was performed to identify the number of cases required to reach proficiency in each item.

RESULTS: Each trainee successfully completed 20 TAMIS training sets. The median total procedure time was 13 min (range, 4-60 min) and stabilized after 15 cases. The median time for specimen removal and wound closure were $3 \mathrm{~min}$ (range, 1-18 $\mathrm{min}$ ) and $10 \mathrm{~min}$ (range, 2-50 min), respectively, and stabilized after seven and fifteen cases, respectively. The mean scores of specimen quality and quality of wound closure were $2.9 \pm 0.9$ and $2.3 \pm 1.1$, respectively, and took 16 and 20 case experiences, respectively, to reach competency in these items. The mean scores of trainee self-confidence and evaluation of technical performance by the supervisor were $2.4 \pm 1.2$ and $2.7 \pm 1.2$, respectively, and took 20 and 17 case experiences, respectively, to reach competency in them.

CONCLUSION: We found that trainees required 15 training sets for the stabilization of procedure time and 16-20 cases to be competent in the TAMIS simulator model. We expect that this simulator model will help surgeons in getting started with TAMIS to overcome the learning curve.
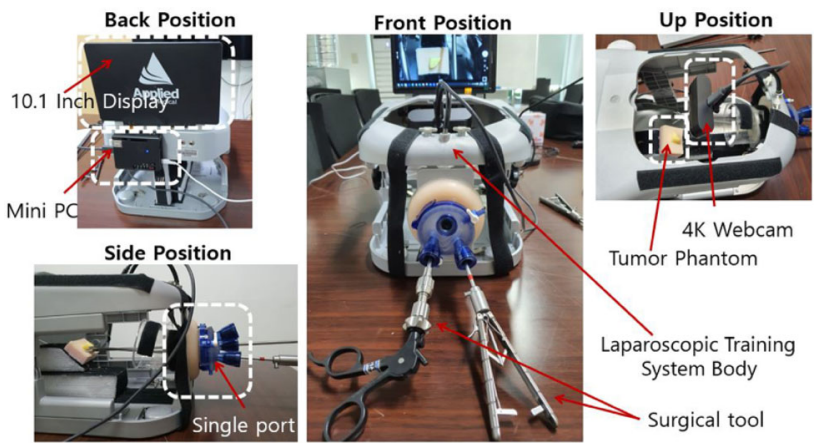
S109

\section{Barriers and Facilitators to Adoption of a Synoptic Operative Note to Improve Communication Across a Statewide Collaborative}

Lia D Delaney, BS ${ }^{1}$; Ryan Howard, $\mathrm{MD}^{2}$; Rhonda Rogers, BNS, RN ${ }^{2}$; Anne P Ehlers, MD, $\mathrm{MPH}^{2}$; Michael Englesbe, $\mathrm{MD}^{2}$; Dana Telem, MD, $\mathrm{MPH}^{2} ;{ }^{1}$ University of Michigan Medical School; ${ }^{2}$ University of Michigan.

Introduction: Efforts to better understand the association between variation in operative technique and patient outcomes following hernia repair have been hindered by inconsistent operative documentation. To address this barrier, we piloted a herniaspecific synoptic operative note as part of a quality improvement effort to create a statewide hernia registry. While communication and data collection of intraoperative variables had improved 6 months after implementation, we sought to further understand the effect of this intervention on surgeon practice, as well as barriers and facilitators to its adoption.

Methods: This quality improvement study was conducted at 8 diverse sites within the Michigan Surgical Quality Collaborative, a statewide quality improvement consortium of 70 hospitals. As part of this consortium, trained nurse abstractors collect perioperative and postoperative data from participating hospitals for select operations. Six months after pilot implementation of a synoptic operative note at these 8 pilot sites, implementation outcomes were assessed via qualitative interviews with nurse abstractors and representative surgeons at the pilot sites. Specifically, feasibility, acceptability, and appropriateness were assessed to determine barriers and facilitators to sustainably scaling the intervention.

Results: Interviews were conducted with 4 surgeons and 4 data abstractors at 6 of the 8 pilot sites. Five of the six sites described subjective improvements in variable completeness since implementation, of which, one site reported surgeon adoption of the synoptic operative note. Two data abstractors observed an increase in the quality of data and completeness of variables, although their affiliated surgeons reported little to no change in practice. The most important facilitator to improved variable communication was consistent communication with abstractors, and continued education about the importance and use of the synoptic operative note. Major barriers to implementation were limitations of the electronic medical record system at the pilot sites, disruptions in routine practice related to COVID-19, and surgeon resistance to adoption of new practices.

Conclusion: Low adoption of the synoptic operative note was reported six months after implementation, despite an increase in the communication of intraoperative hernia variables. These results suggest that consistent and sustained communication with pilot sites, particularly to abstractors, was the most valuable intervention, rather than the synoptic operative note itself. Multipronged, evidence-based strategies that include frequent and constant communication with abstractors who influence surgeons, may be the best approach to improve communication through operative note quality and data collection for a statewide registry.

\section{S110}

Effects of minimally invasive colorectal surgery on PSI 90 across a five-hospital system

Amalia Stefanou; Camden Gardner; Carla Canella; Ilan Rubinfeld; Henry Ford Hospital.

Introduction: The Agency for Healthcare Research and Quality use Patient Safety Indicators (PSI) to gauge patient safety at hospitals. PSI 90 is a weighted combination of several PSIs that are thought most impactful to perioperative patients. This score can affect reimbursement through Medicare and hospital rating. We sought to evaluate differences in PSI 90 occurrences across a five-hospital system for patients who underwent minimally invasive vs open colorectal operations.

Methods: Using ICD-10 codes, a health system administrative database was queried across five participating hospitals for patients who underwent minimally invasive (laparoscopic or robotic) or open colorectal resection or excision surgery between $1 / 2 / 2018-12 / 31 / 2019$. Common patient demographics and health information, along with case characteristics were analyzed with respect to surgical approach and the PSIs composing PSI 90. Statistical relationships between surgical approach and PSIs were investigated using univariate methods, and multivariate logarithmic regression analysis. PSIs of interest are those with the greatest weight in PSI 90: PSI 8 in hospital fracture/fall, PSI 9 perioperative hemorrhage, PSI 11 post-operative respiratory failure, PSI 12 perioperative venous thrombosis, and PSI 13 post-operative sepsis.

Results: There were 1400 operations captured, with 860 (61\%) being open and 540 (39\%) being minimally invasive. Between the minimally invasive and open groups there was no statistically significant difference in regard to patient characteristics outside of biologic sex $(1.36(1.00-1.84)(\mathrm{p}=0.048))$, peripheral vascular disease $(0.60(0.36-0.98)(\mathrm{p}=0.040))$ and COPD $(0.69(0.50-0.96)(\mathrm{p}=0.026))$. MS-DRG diagnosis weight was higher for open operations $(2.52,2.43-2.91)$, compared to minimally invasive operations, $(2.47,1.69-2.52)(\mathrm{p}<0.001))$. LACE score for open colorectal surgery was $16(4-19)$ and $17(15-18)$ for minimally invasive $(\mathrm{p}<0.01)$. PSI 12 occurred in $1.1 \%$ of the open operations and $0 \%$ of the minimally invasive group ( $\mathrm{p}=0.015$ ). PSI 11 occurred $0.9 \%$ in open operations and $0 \%$ of minimally invasive $(\mathrm{p}=0.058)$. Other PSI incidences were not significant between the groups. Within the logistic models there were no statistically significant difference between the two groups created by surgical approach in respect to PSI 3,6 , or 8-15.

Discussion: Understanding PSI 90 and its components are important to enhance perioperative patient care and optimize reimbursement for care provided. We demonstrate that minimally invasive operations, despite providing a known clinical benefit, may not impact scores in the PSI 90. Surgical approach may have little effect on PSI, and there may be other patient and system components that are more important to these outcome measures. 


\section{S111}

\section{OPIOID-FREE ANALGESIA AFTER OUTPATIENT GENERAL SURGERY: A PILOT RANDOMIZED CONTROLLED TRIAL}

Uyen Do ${ }^{1}$; Charbel El Kefraoui, $\mathrm{MSc}^{1}$; Makena Pook $^{1} ;$ Natasha Barone, $\mathrm{MSc}^{2}$; Saba Balvardi, MD $^{3}$; Haley Montgomery ${ }^{4}$; Philip Nguyen-Powanda ${ }^{1}$; Fateme Rajabiyazdi, $\mathrm{PhD}^{1}$; Hiba Elhaj, $\mathrm{MSc}^{4}$; Maxime Lapointe-Gagner ${ }^{1}$; Ghadeer Olleik, $\mathrm{MSc}^{1}$; Pepa Kaneva, $\mathrm{MSc}^{1}$; Alen Antoun, $\mathrm{MD}^{3}$; Nadia Safa, $\mathrm{MD}^{3}$; Elise Di Lena, $\mathrm{MD}^{3}$; Sarkis Meterissian, MD, MSc ${ }^{5}$; Ari Meguerditchian, MD, MSc ${ }^{5}$; Gerald Fried, MDCM, FACS, FRCS ${ }^{1}$; Mohsen Alhashemi, MD, $\mathrm{MSc}^{1}$; Lawrence Lee, MD, $\mathrm{PhD}^{1}$; Gabriele Baldini, MD, MSc $^{6}$; Liane S. Feldman, MDCM, FACS, FRCS ${ }^{1}$; Julio F. Fiore Jr., $\mathrm{PhD}^{1}$; ${ }^{1}$ Steinberg-Bernstein Centre for Minimally Invasive Surgery and Innovation, McGill University Health Centre, Montreal, Quebec, Canada; ${ }^{2} \mathrm{McGill}$ University; ${ }^{3}$ Department of Surgery, McGill University Health Centre, Montreal, Quebec, Canada; ${ }^{4}$ Research Institute of McGill University Health Centre, Montreal, Quebec, Canada; ${ }^{5}$ Department of Surgery and Oncology, McGill University Health Centre, Montreal, Quebec, Canada; ${ }^{6}$ Department of Anesthesia, McGill University Health Centre, Montreal, Quebec, Canada.

Introduction: The overprescription of opioids to surgical patients is recognized as an important driving force behind the opioid crisis. However, the value of prescribing opioids to manage pain following postoperative discharge is still uncertain. This pilot study investigated the feasibility of conducting a full-scale RCT to assess the comparative-effectiveness of opioid analgesia $(\mathrm{OA})$ versus opioid-free analgesia (OFA) after outpatient general surgery (ClinicalTrials.gov: NCT04254679).

Methods: This pragmatic, assessor-blind, pilot RCT included adult patients undergoing outpatient abdominal and breast surgeries at two tertiary hospitals (target $\mathrm{n}=$ 80 , anticipated attrition 15\%). Prior to discharge, patients were randomized 1:1 to receive OA [around-the-clock non-opioids (acetaminophen and NSAIDs) and opioid tablets for breakthrough pain] or OFA (around-the-clock non-opioids and increasing doses and/or adding non-opioid drugs for breakthrough pain). Patients with contraindications to the trial drugs or complications requiring hospital stay were excluded. The study primarily focused on a priori RCT feasibility criteria: $>70 \%$ screened patients meet eligibility criteria, $>50 \%$ eligible patients are randomized and $>80 \%$ randomized patients complete follow-up. Other outcomes included 7-day pain severity and interference (Brief Pain Inventory), analgesics intake, adverse events, and satisfaction with pain management (5-point scale). Data were analyzed using descriptive statistics and effect-estimates of the outcomes considered for the full-scale RCT

Results: Of 224 patients assessed for eligibility (January-September 2020), 164 met inclusion criteria $(73 \%)$ and 93 consented for randomization $(57 \%)$. Twelve patients were excluded pre-randomization [surgery during COVID-19 restrictions $(n=5)$ or after trial completion $(\mathrm{n}=7)$ ]; five were excluded post-randomization [complication requiring hospital stay $(\mathrm{n}=3)$, contraindication to NSAIDs identified before discharge $(n=2)$ ]. Seventy-six patients (39 OA and 37 OFA) were included in the intention-to-treat analysis [mean age 55.5, 66\% female, 53\% abdominal surgery (48\% laparoscopic), $47 \%$ breast surgery (50\% sentinel node biopsy, $16 \%$ axillary dissection)]. All patients completed the 7-day follow-up. Pain intensity and interference were comparable between groups (Figure). Twenty-three OA patients did not take opioids (59\%). One OFA patient received an opioid prescription after discharge. Common adverse events were nausea (OA $21 \%$ vs. OFA $16 \%$ ), vomiting ( $8 \%$ vs. $3 \%)$ and constipation $(41 \%$ vs. $32 \%)$. Satisfaction with pain management (satisfied/ very satisfied) was reported by 37 patients in the OA group (95\%) and 34 in the OFA group $(92 \%)$.

Conclusion: This pilot study supports the feasibility of a full-scale RCT to assess the comparative-effectiveness of opioid versus opioid-free analgesia after outpatient general surgery. A robust, adequately powered RCT has the potential to contribute practice-changing evidence to mitigate postoperative opioid overprescribing.

\begin{tabular}{|c|c|c|c|c|c|c|c|}
\hline \multicolumn{4}{|c|}{ Pain severity } & \multicolumn{4}{|c|}{ Pain interference } \\
\hline Day & $\begin{array}{l}\text { Favors } \\
\text { Opioid-tree }\end{array}$ & $\begin{array}{l}\text { Favors } \\
\text { Opioids }\end{array}$ & Mean difference with $95 \% \mathrm{Cl}$ & Day & $\begin{array}{l}\text { Fevors } \\
\text { Opioid-tree }\end{array}$ & $\begin{array}{l}\text { Favors } \\
\text { Opioids }\end{array}$ & Mean difference with $95 \% \mathrm{C}$ \\
\hline POD 1 & & & $\cdot 0.02[\cdot 0.92,0.88]$ & POD 1 & & & $0.06[-1.14,1.27]$ \\
\hline POD 2 & & & $-0.28\left[\begin{array}{ll}-1.30, & 0.73]\end{array}\right.$ & $\mathrm{POO} 2$ & & & $-0.24[-1.28,0.81]$ \\
\hline POD 3 & & & $-0.35[-1.22, \quad 0.52]$ & POO 3 & • & & $-0.35[-1.37,0.68]$ \\
\hline POD 4 & & & $-0.43[-1.21,0.35]$ & POO 4 & - & & $-0.69[-1.72,0.35]$ \\
\hline POD 5 & & & $-0.31\left[\begin{array}{ll}-1.02, & 0.39\end{array}\right]$ & P00 5 & & & $-0.27[-1.09,0.55]$ \\
\hline P0D 6 & & & $-0.39\left[\begin{array}{ll}-1.06, & 0.28\end{array}\right]$ & P00 6 & - & & $-0.58[-1.39,0.22]$ \\
\hline POD 7 & . & & $-0.38[-1.01,0.26]$ & POD 7 & & & $-0.25[-1.14,0.63]$ \\
\hline-2 & -1 & 0 & $\frac{1}{1}$ & -2 & -1 & & \\
\hline
\end{tabular}

\section{S112}

\section{Analysis of Exhaled Volatile Organic Compounds: A New Frontier in Colon Cancer Screening and Surveillance}

Alexandra Allard-Coutu, Hon, BSc, MDCM, FRCSC ${ }^{1}$; Kevin Singh, Hon, BSc, MDCM, FRCPC $^{2}$; André Lamontagne ${ }^{3}$; Yves Gamache ${ }^{3}$; Barbara Heller, Hon, BSc, MD, FRCSC $1{ }^{1}$ McMaster University; ${ }^{2}$ University of Toronto; ${ }^{3}$ eTraceMedical.

BACKGROUND: Neoplastic processes cause distinct and immediate changes to the body's metabolism, creating unique patterns in the volatile organic compounds (VOCs) being produced and released. Unique VOC profiles have been shown to be diagnostic for certain cancers. Exhaled breath VOC analysis has potential as a pointof-care tool for screening for colon cancers in a single sample. This technology can also be used to monitor response to treatment and applied to post treatment cancer surveillance protocols.

METHODS: An exhaled breath VOC analyzer was developed based on ASDevices and eTrace Medical patented technology. Proof of principle experiments established a detection limit and sensitivity/specificity of the Epd sensing technology. A casecontrol trial is in process to validate the device using 25 exhaled VOCs previously shown to be diagnostic of colon cancer. VOCs aspirated from the colon during colonoscopy in patient with known colon cancer will be also be sampled and compared to the VOC profile of exhaled breath. The ability to reliably detect colon cancer will be evaluated by receiver operating characteristic curve analysis followed by cross-validation by leave-one-out method and stepwise logistic regression analysis.

PRELIMINARY RESULTS: Using representative biomarkers in exhaled breath proof of principle testing confirmed the sensitivity and selectivity of the sensing technology based on a proprietary gas chromatography (GC) method from eTrace Medical (patent pending). Protocol validation included testing of ASDevices technologies (pre-treatment and concentrator, iMov GC platform, uInProve GC valve and Epd sensing technologies) (FIGURE 1A). Targeted molecules (sulfurs, benzene, toluene, xylene) were contained in an air matrix to simulate exhaled breath. This validation system demonstrated that the device can reliably measure targeted molecules at levels within $10 \mathrm{ppb}$ of their nominal value (FIGURE 1B). The detection limit, based on $3 \times$ signal:noise ratio, was between $200-700$ ppt. The system's sensitivity can be further adapted via sample concentration and pretreatment

CONCLUSION: Implementation of VOC analysis for accessible screening and early detection of colon cancer could improve patient outcomes and decrease breastcancer related death, as well as the global disease burden. We propose a proof-ofconcept case-control study to determine the sensitivity and specificity of exhaled breath analysis using a novel point-of care-tool for the diagnosis of breast cancer using previously described VOC breathprints.
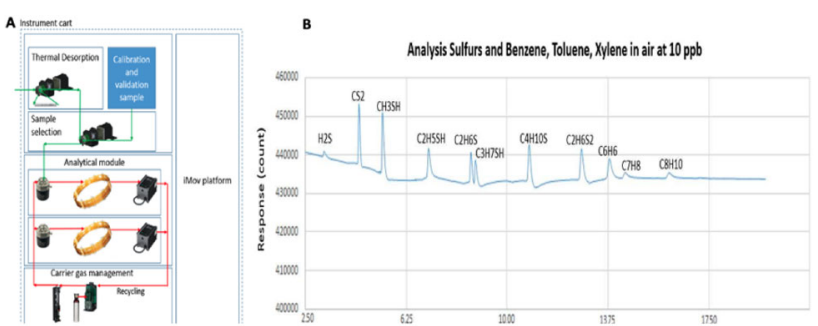

FIGURE 1:

A Components of the prototyp

Targeted molecules (sulfurs, benzene, toluene, xylene) were contained in an air matrix to simulate exhaled breath. This validation system demonstrated that the nominal value. The detection limit, based on $3 x$ signal: noise ratio, was between 
S113

An assessment tool for oncologic quality of robotic proctectomy

Dinallo Anthony, MD; Terrah J Paul Olson, MD; Patrick S Sullivan, MD; Virginia O Shaffer, MD; Jennifer L Williams, MD; Balch C Glen, MD; Rupji Manali, MS; Liu Yuan, PhD; Seth A Rosen, MD; Emory University.

Introduction: The National Accreditation Program for Rectal Cancer requires a synoptic surgical report. Operative reports, however, may not accurately represent surgical events. The pathology report, which is retrospective in nature, is therefore the primary means of assessing quality of rectal cancer surgery. This may be problematic, as more cancer procedures are performed minimally invasively, and specimen injury can occur during extraction through a small incision. Validated scorecards are used to assess surgeon competence, however none specifically assess oncologic quality of an operation. Our primary goal was to create a scorecard to assess oncologic quality of robotic proctectomy for rectal cancer and evaluate its interobserver reliability.

Methods and Procedures: We reviewed literature regarding techniques and goals of rectal cancer surgery. Then, using Delphi method, six colorectal surgeons arrived at consensus on the steps required for curative rectal cancer surgery. Using the results of our iterative process, as well as input from two biostatisticians, we created the Emory University Proctectomy Assessment Tool (EUPAT). From May 1, 2019 through May 1, 2020, all robotic proctectomies were recorded and 40 videos were randomly chosen for editing. Every 30 days, surgeons are sent edited videos to review using the EUPAT. Answers are recorded anonymously. Intra-class correlation coefficients were reported along with $95 \%$ CI as a measure of interrater reliability based on two-way random effects, consistency, and average using the 'icc' R package.

Results: The EUPAT includes 15 questions that assess quality of mesorectal excision, adjacent organ or nerve injury, location of artery ligation and quality of distal margin. Original videos were a mean of $194(S D \pm 64)$ minutes, editing required a mean of $94(\mathrm{SD} \pm 50)$ minutes, and final videos averaged $23(\mathrm{SD} \pm 6)$ minutes. Interim analysis was performed after 60 EUPATs were completed (10 videos X 6 surgeons). We found good inter-rater reliability coefficients for location of inferior mesenteric artery ligation $(0.79[0.49-0.94])$, distal margin adequacy $(0.78$ [0.47-0.94]) and extent of bleeding (0.75 [0.39-0.93]), and moderate inter-rater reliability coefficients for presacral nerve preservation $(0.69$ [0.26-0.91]), distal margin consistency $(0.68[0.18-0.92])$, anterior plane dissection $(0.65[0.16-0.9])$, anterior envelope appearance $(0.72[0.33-0.92])$ and right envelope appearance $(0.65$ [0.17-0.9]).

Conclusions: We created a scorecard to assess oncologic quality of proctectomy for rectal cancer. After interim analysis, our scorecard demonstrated evidence of good and moderate inter-rater reliability. We will continue collecting data regarding interrater reliability as well as intra-rater reliability.

\section{S114}

\section{SAME DAY DISCHARGE DOES NOT LEAD TO WORSE OUTCOMES IN PATIENTS UNDERGOING UNCOMPLICATED HELLER MYOTOMY, NISSEN AND TOUPET FUNDOPLICATION, AND PARAESOPHAGEAL HERNIA REPAIR}

Miriam Brazer, MD; Dylan Russell, MD; Scott Nguyen, MD; Brooke Pati, MD; Christopher Yheulon, MD; Tripler Army Medical Center.

Introduction: With the advancement of minimally invasive surgical techniques surgeons have moved away from open elective foregut surgeries, specifically Heller myotomy, Nissen and Toupet fundoplication, and paraesophageal hernia repairs. This has led to a decrease in patient recovery time and post-operative pain. Patients without life threatening comorbidities who undergo uncomplicated hiatal operations are typically discharged on post-operative day one. Despite studies demonstrating the safety of same day discharge in the appropriate patient population, ambulatory surgery has yet to be established as practice norm for patients undergoing uncomplicated hiatal surgery.

Methods and Procedures: The ACS-NSQIP database was queried from 2005-2018 for patients who had undergone Heller myotomy, Nissen and Toupet fundoplication, and paraesophageal hernia repairs with and without mesh. Patients with concurrent procedures were not included in the analysis. A 3:1 (discharge on post-operative day one:same day discharge) propensity score match was performed for age, sex, ASA classification, operative time, smoking history, hypertension requiring medication, and procedure performed. The primary endpoints in this study were number and severity of complications as classified by the Clavien-Dindo Classification, readmissions, and return to the operating room.

Results: 6893 patients who met the inclusion criteria were identified, $696(10.1 \%)$ of which were discharged on the day of surgery. Patients who were discharged on postoperative day one were matched at a 3:1 ratio producing 2088 comparisons. There was no difference in complications rates as classified by the Clavien-Dindo Classification $(\mathrm{p}=0.264)$, number of readmissions $(\mathrm{OR} 0.849,95 \%$ CI $0.522-1.419, \mathrm{p}=$ $0.534)$, or number of patients who returned to the operating room (OR 1.15, 95\% CI $0.531-2.761, \mathrm{p}=0.855$ ) between the two groups.

Conclusion: While most patients who undergo minimally invasive Heller myotomy, Nissen and Toupet fundoplication, and paraesophageal hernia repairs are discharged on post-operative day one, there is growing evidence to support discharging these patients on the day of surgery if their surgery was uncomplicated and they do not have life threatening comorbidities. Discharge on the day of surgery appears to be safe and is not associated with increased rates of complication, readmission, or return to the operating room. 
S115

\section{IS MINIMALLY INVASIVE SURGERY FOR LARGE GASTRIC GIST SAFE? A COMPARATIVE ANALYSIS OF SHORT- AND LONG-TERM OUTCOMES}

Eduardo A Vega, MD; Onur C Kutlu, MD; Omid Salehi, MD; Vera Kazakova, MD; Sylvia V Alarcon, MD; Olga Kozyreva, MD; Claudius Conrad, MD, PhD, FACS, FSSO; St Elizabeth's Medical Center.

INTRODUCTION: While minimally invasive surgery (MIS) is frequently utilized to surgically remove small gastric gastrointestinal stroma tumors (GIST), using MIS for tumors $\geq 5 \mathrm{~cm}$ remains an area of controversy. In this context, open surgery is recommended by national guidelines due to concerns of feasibility, safety and oncological outcomes. This study investigates the perioperative and long-term outcomes of MIS for T3 gastric GIST measuring 5-10 cm in size.

METHODS AND PROCEDURES: The National Cancer Database (NCDB) 2017 was used as the data source. Patients undergoing gastric GIST (ICD O-3 8936) with tumor $5-10 \mathrm{~cm}$ were identified. Inclusion criteria were known stage, size, comorbidities, grade, lymphovascular invasion, type of surgery, approach, conversion info, margin status, neoadjuvant and adjuvant treatment, hospital stay, readmission, 30and 90-day mortality, complete follow-up, type of institution and hospital gastric surgery case volume. Binary logistic regression was used for 30- and 90-day mortality, readmission and margin status. Linear regression models were used for hospital stay. Kaplan Meier survival analysis and Cox regression models were used to identify the impact of MIS surgery on overall survival (OS).

RESULTS: 3765 patients met inclusion criteria, $49.9 \%$ were male. Mean tumor size was $67.1 \mathrm{~mm}, 22.8 \%$ underwent MIS, and $73.8 \%$ underwent open gastrectomy, $3.4 \%$ were converted to open. Median hospital stay was 4 days for MIS, 5 days for MIS converted to open and 6 days for open gastrectomy groups; Linear regression model significantly favored MIS (beta $0.169, \mathrm{p}<0.001)$. 30-day mortality: $0.9 \%$ MIS vs. $1.6 \%$ open (OR $1.114, \mathrm{p}=0.033$ ), 90-day mortality: $1.4 \%$ MIS $2.2 \%$ vs. open (OR $1.109 \mathrm{p}=0.036)$. R1 margin rate was $2.9 \%$ MIS vs. $3.1 \%$ open $(\mathrm{p}=0.143)$ Unplanned readmission rate was $2.4 \%$ MIS vs. $4.0 \%$ open (OR $1.101, p=0.034$ ). Cox regression models for OS showed no difference in survival between MIS to open and open approaches $(\mathrm{p}=0.137$, HR 0.808).

CONCLUSION: This retrospective, comparative analysis shows that MIS approach for large gastric GIST $>5 \mathrm{~cm}$ is oncologically safe with improved postoperative morbidity (shorter hospital stay, lower readmission rate, improved 30- and 90-day mortality rates, similar margin status). Moreover, as MIS vs. open surgery provides similiar long-term survival, national guidelines may need to incorporate this new information to reflect the data presented here.

\section{S116}

\section{SURGICAL MANAGEMENT OF BARRETT'S ESOPHAGUS IS SUPERIOR TO MEDICAL THERAPY FOR DISEASE PROGRESSION AND REGRESSION: A SYSTEMATIC REVIEW AND META-ANALYSIS}

Hillary Wilson, RD; Warren Sun, MD; Jerry T Dang, MD; Uzair Jogiat, MD Valentin Mocanu, MD; Janice Y Kung, BCom, MLIS; Noah J Switzer, MD, MPH; Clarence K Wong, MD; Shahzeer Karmali, MD, MPH; University of Alberta.

Introduction: Barrett's esophagus (BE) is defined as the presence of intestinal metaplasia within the esophageal epithelium and presents the greatest risk factor for development of esophageal adenocarcinoma. Fundoplication and medical management are the current mainstays for the management of BE, however, evaluation of long-term outcomes between these treatments are lacking. Our aim was to perform a systematic review and meta-analysis to evaluate the efficacy of fundoplication compared to medical therapy in patients with BE.

Methods and Procedures: A comprehensive search in MEDLINE, EMBASE, Scopus, Web of Science and Cochrane Library databases was performed on December 16, 2019, by a university librarian. Two authors independently screened full-text articles. Inclusion criteria included human participants, age $\geq 18, \mathrm{~N} \geq 5$, diagnosis of BE prior to fundoplication, and primary fundoplication. Studies were excluded if they did not evaluate our primary outcomes of interest - histopathologic disease progression and regression. A meta-analysis using the RevMan 5.4.1 software was performed with a Mantel-Haenszel, random-effects model.

Results: A total of 6872 studies were retrieved after initial search with 9 studies $(n=$ 1 randomized trial, $n=7$ prospective cohort studies, $n=1$ retrospective cohort study) meeting final inclusion criteria. Of included studies, 28,535 (96\%) patients received medical management while $1,164(4 \%)$ received surgical management. Patients receiving surgical therapy were less likely to have non-dysplastic BE (70\% vs. $78 \%$ ) and more likely to have low grade dysplasia (29\% vs. $21 \%$ ) than those receiving medical therapy. Medical management included proton pump inhibitors (807 patients; $\mathrm{n}=6$ studies), $\mathrm{H} 2$ receptor antagonists ( 40 patients; $\mathrm{n}=3$ studies), combination therapy (43 patients; $\mathrm{n}=1$ study) and not specified (27,635 patients; $\mathrm{n}=1$ study). When specified, Nissen fundoplication was the most commonly performed type of fundoplication $(\mathrm{n}=356,67 \%)$. Median length of follow up ranged from 18-72 months. Meta-analysis revealed that fundoplication was associated with improved histopathologic regression (OR 6.34; 95\% CI 3.29-12.19; $\mathrm{p}<0.000001$; $\mathrm{I} 2=0 \%$ ) and less disease progression (OR $0.34 ; 95 \%$ CI $0.12-0.96 ; \mathrm{p}=0.04 ; \mathrm{I} 2=$ $0 \%$ ) in comparison to standard medical therapy alone.

Conclusion: Surgical management of BE was associated with significantly higher rates of histopathologic disease regression and less progression to dysplasia compared to standard medical therapy alone. Further large randomized controlled trials which directly compare medical management and surgical intervention are required to further delineate the optimal delivery and timing of these interventions.

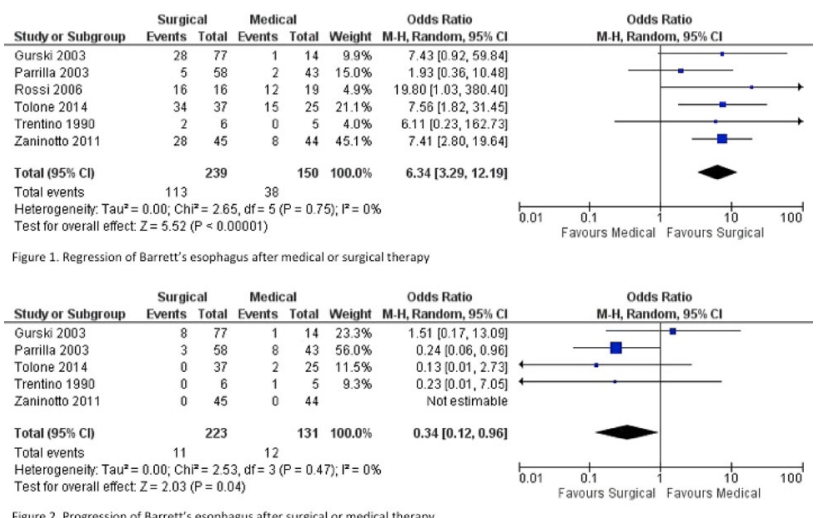


S117

\section{LONG-TERM OUTCOMES OF NISSEN FUNDOPLICATION VERSUS ROUX-EN-Y GASTRIC BYPASS FOR PRIMARY MANAGEMENT OF GASTROESOPHAGEAL REFLUX DISEASE IN OBESE PATIENTS}

Jennifer S Colvin; Anahita D Jalilvand; Phoebe Um; Sabrena F Noria; Bradley J Needleman; Sean M O'Neill; Kyle A Perry; Ohio State University Wexner Medical Center.

INTRODUCTION: Laparoscopic Nissen fundoplication (LNF) is the standard surgical treatment for gastroesophageal reflux disease (GERD); however, its efficacy in obese patients remains controversial. Specifically, concerns regarding long-term symptom control in the setting of obesity has driven interest in laparoscopic Rouxen-Y gastric bypass (RYGB) as a primary reflux operation for these patients. In this retrospective cohort study, we compared the impact of LNF and RYGB on diseasespecific quality of life and anti-secretory medication use in morbidly obese patients with GERD.

METHODS AND PROCEDURES: Patient data are maintained in an IRB approved foregut surgery database. We included all patients with a BMI greater than $35 \mathrm{~kg} / \mathrm{m} 2$ undergoing primary anti-reflux surgery between 2015 and 2019. Followup was completed via telephone interview at a median interval of 42 [19-57] months. Outcome measures included GERD health-related quality of life (GERDHRQL) scores, proton pump inhibitor (PPI) use, endoscopic intervention, and satisfaction with the procedure. Data are expressed as mean $\pm \mathrm{SD}$, median (IQR), or number $(\%)$ as appropriate. A p-value of $<0.05$ was considered statistically significant

RESULTS: Forty-four patients underwent primary anti-reflux surgery during the study period including $20 \mathrm{LNF}$ and $24 \mathrm{RYGB}$. RYGB patients were younger ( $42.8 \pm$ 11.4 versus $51.5 \pm 15.4, \mathrm{p}=0.04)$ and had higher BMI $(45.5[39.2-49.8]$ vs 39.0 $[38.5-40.4], p=0.004)$ compared LNF patients. ASA classification, DeMeester score, presence of hiatal hernia or Barrett's esophagus, baseline PPI use, and baseline GERD-HQRL scores did not differ between groups. Operative time, need for reoperation, and readmission rates were comparable, but 5 patients $(25 \%)$ developed a marginal ulcer following RYGB, and $4(20 \%)$ required endoscopic dilation compared to $3(12.5 \%)$ following LNF $(\mathrm{p}=0.684)$. GERD-HRQL scores improved from 36 [28-43] at baseline to 9 [3.5-16.5] at follow-up following RYGB $(\mathrm{p}<0.001)$ and from 29 [16-37] to $1.5[0-14]$ after LNF $(\mathrm{p}<0.001)$. GERD-HRQL scores $(\mathrm{p}=$ $0.07)$ and PPI useage $(58 \%$ versus $28 \%, \mathrm{p}=0.10)$ and need for redo surgery $(0 \%$ versus $6 \%, \mathrm{p}=0.69$ ) did not differ significantly between groups. Eighty-three percent of patients reported satisfaction with RYGB compared to $78 \%$ following LNF ( $p$ $=0.71$ )

CONCLUSIONS: RYGB and LNF produced similar long-term improvements in disease-specific quality of life following primary anti-reflux surgery with similar rates of complications, side effects, and need for reoperation. This demonstrates that RYGB and LNF represent reasonable options for surgical management of GERD in obese patients, however, the optimal procedure in an individual patient will be heavily influenced my their medical comorbidities and personal preferences.

\section{S118}

Endoscopic Sleeve Gastroplasty for Treatment of Obesity is Safe and Effective: Interim Results from a Prospective National Registry

Jennifer Maranki, MD, MSc ${ }^{1}$; Steven Schwaitzberg, MD, FACS ${ }^{2}$; Michael L Kochman, $\mathrm{MD}^{3}$; ${ }^{1}$ Penn State Hershey Medical Center; ${ }^{2}$ University at Buffalo-The State University of New York; ${ }^{3}$ University of Pennsylvania.

Background: Endoscopic sleeve gastroplasty (ESG) has emerged as a treatment option for obesity, but questions remain about its overall safety and efficacy. We report weight loss and adverse events with 6-month follow-up.

Methods: In this national, prospective, multicenter registry aimed at capturing current use of trans-orifice endoscopic suturing applications, consecutive patients undergoing ESG for treatment of obesity were evaluated.

Results: A total of 80 patients from six sites were enrolled, 62 were female and 18 male, average age of 47.8 years. Baseline body weight was $105.7 \pm 22.7 \mathrm{~kg}$. Percent total body weight loss (\%TBWL) at 30 days, 3 months, and 6 months was $7.83 \pm$ $4.33 \%, 11.41 \pm 3.92 \%$, and $13.86 \pm 6.88 \%$, respectively. Immediate complications occurred in 6 patients $(7.5 \%)$, of which $4(5 \%)$ were considered clinically relevant by the investigators, and consisted of nausea and/or vomiting, abdominal pain, and/or fever. All required either additional medications (1), an ED visit (1), or a hospitalization (2). There were $3(3.75 \%)$ delayed complications, all of which occurred within 30 days. One developed nausea and vomiting, one experienced dehydration, and one developed a leak from dietary indiscretion and was treated conservatively. No patient underwent additional therapeutic endoscopic, IR procedures or surgery. Conclusion: 1. ESG is promising minimally invasive technique for the treatment of obesity, with a favorable adverse event profile. 2. Additional follow-up will determine long-term efficacy.

\section{S119}

Association of Program-Specific Variation in Bariatric Surgery Volume for Medicaid Patients and Access to Care: A Tale of Inequality?

Amy E Somerset, $\mathrm{MD}^{1}$; Aaron J Bonham, $\mathrm{MSc}^{2}$; Arthur M Carlin, $\mathrm{MD}^{3}$; Jonathan F Finks $\mathrm{MD}^{2}$; Amir A Ghaferi $\mathrm{MD}^{2}$; Michael H Wood, $\mathrm{MD}^{4}$; Oliver A Varban, $\mathrm{MD}^{2}$; ${ }^{1}$ Beaumont Health; ${ }^{2}$ University of Michigan; ${ }^{3}$ Henry Ford; ${ }^{4}$ Detroit Medical Center.

BACKGROUND: Requirements for bariatric surgery coverage vary considerably by insurance type which can impact utilization. To date, it is unclear if Medicaid patients who qualify for bariatric surgery experience limited access to surgery as a result of program-specific variation in volume and distribution of cases based upon insurance type. The objective of this study was to evaluate the current structure of bariatric programs to identify the distribution of patients.

METHODS: Using a state-wide bariatric-specific data registry that includes 39 programs performing 97,207 cases between 2006 and 2020, bariatric programs were stratified into quartiles according to the percentage of Medicaid cases performed. Patient characteristics, postoperative outcomes and wait times were compared between programs designated as high or low volume based on percent of Medicaid patients.

RESULTS: A total of 4,780 (4.9\%) bariatric operations were performed for patients with Medicaid during the study period. Program-specific distribution of Medicaid cases varied between $0.69 \%$ to $22.4 \%$. Programs in the top quartile for Medicaid patients $(\mathrm{n}=11)$ performed an overall mean of $13 \%$ Medicaid with a total volume of 18,885 cases. Programs in the bottom quartile $(\mathrm{n}=11)$ performed a mean $1 \%$ of Medicaid cases with a total bariatric volume of 32,447 . Patients undergoing surgery at high-volume Medicaid programs were more likely to be Black $(20.2 \%$ vs $13.5 \%, \mathrm{p}<0.0001)$ and had higher rates of diabetes $(35.1 \%$ vs $29.5 \%$, p $<0.0001)$, hypertension $(55.1 \%$ vs $49.6 \%$, p $<$ $0.0001)$, asthma (24.9 vs $19.3 \%, \mathrm{p}<0.0001)$, sleep apnea $(48.4$ vs $42.0 \%$, p < $0.0001)$, and hyperlipidemia $(47.6 \%$ vs $45.2 \%, \mathrm{p}<0.0001)$ despite clinically similar age and preoperative body mass index (BMI). In addition, high volume Medicaid programs had higher rates of complications ( $8.4 \%$ vs $6.6 \%,<0.0001)$, extended length of stay $(5.6 \%$ vs $4.0 \%, \mathrm{p}<0.0001)$, Emergency Department visits $(8.1 \%$ vs $6.5 \%, \mathrm{p}<0.0001)$ and readmissions $(4.7 \%$ vs $3.9 \%, \mathrm{p}<0.0001)$. Median time from initial evaluation to surgery date was significantly longer for patients with Medicaid than with private insurance (216 days vs 136 days, $\mathrm{p}<0.0001$ ).

CONCLUSIONS: Bariatric surgery programs vary widely in their case volume of Medicaid patients. Centers with high Medicaid case volumes include more Black and low-income patients. These centers also perform fewer operations with substantially longer wait times for Medicaid patients compared to private insurances, leading to a significant disparity in access to care. Moreover, high-volume Medicaid programs treat patients with greater disease severity and more postoperative resource utilization, which may disincentivize any efforts to improve equality in access to care. 
S120

\section{RACIAL INEQUITY IN PRE-OPERATIVE REQUIREMENTS BASED ON INSURANCE AMONG ELIGIBLE PATIENTS FOR BARIATRIC SURGERY}

Ava F Bryan, MD, AM; Caridad McRae, MBA; Mustafa Hussain, MD; Yalini Vigneswaran; The University of Chicago, Department of Surgery.

Introduction: Although Black communities in the United States have a large and disproportionate burden of obesity, it is well established that weight loss surgery is largely performed on non-Hispanic white patients. Differences in insurance-specific pre-operative requirements contributing to these disparities has not been investigated, although the effectiveness of supervised weight loss (SWL) programs has been questioned. Medicaid requires strict adherence to six months of SWL, while private insurance requirements are variable but less rigid. Serving a large Black community, we hypothesized that a racial difference in payer mix could represent a racial disparity in access to weight loss surgery.

Methods: Using an internal institutional all-payer claims database from August 1, 2015 until August 31, 2020 we categorized all patients evaluated in an outpatient clinic under a bariatric surgery provider by payer type (public vs. private). Race and ethnicity were filtered for non-Hispanic white or Black. The resulting data were compared for independence using Chi-square test of independence.

Results: Of 3982 total patients seen in the bariatric surgery outpatient clinics, 2598 $(65 \%)$ were Black and $933(23 \%)$ were non-Hispanic white. The remaining $12 \%$ were Hispanic/Latino white, more than one race, Native Hawaiian/Other Pacific Islander, or of unknown race/ethnicity; these patients were not included in the final analysis. Breakdown of insurance payer type by race is presented in Table 1 . Analysis yielded a Chi-square of $219(\mathrm{p}<0.001)$, indicating a significant association between race and insurance status among the patients seen for bariatric surgery evaluation in our clinic.

Conclusions: Non-Hispanic white patients seen in bariatric clinic were significantly more likely than Black patients to have private insurance, obviating them from extensive, perhaps perfunctory requirements in the absence of a provider requesting such clearance based on clinical judgment. This finding represents a systemic barrier to care experienced by Black patients. We believe that in the absence of clinical data to support the benefit of such preoperative requirements, these unnecessary barriers should be eliminated in order to deliver equitable healthcare in the United States.

Table 1: Insurance distribution by race

\begin{tabular}{llll}
\hline & Private n $(\%)$ & Public n $(\%)$ & Total \\
\hline Black & $1460(56)$ & $1138(44)$ & 2598 \\
White & $779(83)$ & $154(17)$ & 933 \\
Total & 2239 & 1292 & 3531 \\
\hline
\end{tabular}

\section{$\mathbf{S 1 2 2}$}

\section{Racial Variation in Baseline Characteristics and Wait Times Among Patients Undergoing Bariatric Surgery: Not as Simple as Black and White}

Mary R Shen, MD, MS ${ }^{1}$; Aaron Bonham, $\mathrm{MSc}^{1}$; Jonathan $\mathrm{F}_{\text {Finks }}{ }^{1}$; Amir Ghaferi, MD, MS, FACS, FASMBS ${ }^{1}$; Arthur Carlin, MD, FASMBS ${ }^{2}$; Oliver Varban, MD FACS, FASMBS ${ }^{1} ;{ }^{1}$ University of Michigan; ${ }^{2}$ Henry Ford Health System.

Background: Although bariatric surgery is the most effective treatment for obesity and weight-related comorbid diseases, utilization rates are disproportionately low among non-white patients. We sought to understand if variation in baseline characteristics or access to care exists between white and non-white patients.

Methods: Using a statewide bariatric-specific data registry, we evaluated all patients who underwent bariatric surgery between 2006 and 2020 and also completed a preoperative baseline questionnaire, which included a question about self-identification of race $(n=73,141)$. Patient characteristics, comorbidities and time from initial presentation to surgery were compared among racial groups.

Results: A total of $18,741(25.5 \%)$ patients self-identified as non-white, which included Black/African American $(\mathrm{n}=11,904)$, Hispanic $(\mathrm{n}=3,448)$, Asian $(\mathrm{n}=121)$, Native Hawaiian/Pacific Islander $(n=41)$, Middle Eastern $(n=164)$, Multiple $(n=2,047)$ and Other $(n=608)$. Non-white males were the least represented group, accounting for only $4 \%$ of all bariatric cases performed. Non-white patients were more likely to be younger (43.0 years vs 46.6 years, $\mathrm{p}<0.0001)$, disabled $(16 \%$ vs $11.4 \%, \mathrm{p}<0.0001)$ and have Medicaid $(8.4 \%$ vs $3.8 \%, \mathrm{p}<0.0001)$ when compared to white patients, despite having higher rates of college education $(78.0 \%$ vs $76.6, p<0.0001)$. In addition, median time from initial evaluation to surgery was also longer among non-white patients (157 days vs 127 days, $\mathrm{p}<0.0001$ ), despite having higher rates of patients with a body mass index (BMI) above $50 \mathrm{~kg} / \mathrm{m} 2(39.0 \%$ vs $33.2 \%, \mathrm{p}<0.0001)$.

Conclusions: Non-white patients undergoing bariatric surgery represent an extremely diverse group of patients with more socioeconomic disadvantages and longer wait times when compared to white patients in spite of presenting with higher rates of severe obesity. Current guidelines and referral patterns for bariatric surgery may not be equitable and needs further examination when considering the management of obesity within a diverse population. 
S123

\section{Robotic vs. Laparoscopic Gastric Bypass: Comparisson of Postoperative Pain and Outcomes}

Mark J Dudash, MD; Shengxuan Wang, MS; Alicia Johns, PhD; Ryan Colonie, MS; Alexandra Falvo, MD; Ryan Horsley, DO; Jon Gabrielsen, MD, FACS; Mustapha Daouadi, MD; Anthony T Petrick, MD, FACS; David M Parker, MD, FACS; Geisinger Medical Center.

Introduction: The benefit of the laparoscopic approach on postoperative pain and opioid use is well established. There are currently no studies to evaluate postoperative pain and opioid use in robotic bariatric surgery. Robotic port sites have a "remote-center" that is purported to minimize tissue torque on the abdominal wall and thus pain. We aimed to determine if patients who underwent Roux-en-Y gastric bypass by a robotic approach (RA-RYGB) had lower postoperative pain scores and required less opioids than their laparoscopic (L-RYGB) counterparts. Postoperative outcomes including length of stay, operative time, and readmissions were also included.

Methods and Procedures: This was a retrospective cohort study examining data from five surgeons at a single tertiary academic medical center. Adult patients who underwent L-RYGB or RA-RYGB between 5/1/2018-10/31/2019 were included. Cases with concomitant hernia repair $(n=18)$, chronic opioid use $(n=75)$, and those who did not receive a TAP block or multimodal pain control $(n=36)$ were excluded. Baseline demographics were compared. Inpatient and outpatient opioid usage in Morphine Milligram Equivalents (MME) as well as pain scores (10-point Likert scale) were compared between surgical approaches.

Results: There were a total of 573 RYGB patients included (462 L-RYGB) and 111 RA-RYGB). Median and maximum inpatient pain scores were similar for L-RYGB and RA-RYGB ( 3.0 vs $3.1, p=0.878 ; 7.0$ vs $7.0, p=0.688$ ). Median inpatient opioid as well as maximum single day use was also similar for L-RYGB and RA-RYGB (40.0 MME vs. $42.0, \mathrm{p}=0.671 ; 30.0$ vs $30.0, \mathrm{p}=0.648$ ). Patient reported pain at 2-week follow up was significantly higher for RA-RYGB $(68.1 \%)$ compared to L-RYGB $(55.6 \%)(\mathrm{p}=0.030)$. No significant difference in the receipt of outpatient narcotic prescription was noted between L-RYGB and RA-RYGB (50.2\% vs. $42.3 \%$, $\mathrm{p}=0.136$ ). There was no significant difference in MME outpatient opioids at 2 weeks (L-RYGB 30.0 MME vs. 33.8, p = 0.854). The robotic approach was associated with a higher 30 day readmission and longer operative times compared to the laparoscopic approach $(6.3 \%$ vs $13.5 \%, \mathrm{p}=0.010$; median length of $144.5 \mathrm{~min}$ vs $200.0 \mathrm{~min}, \mathrm{p}<0.001)$. The robotic approach had a shorter length of stay $(\mathrm{p}=0.023)$ but this was not clinically significant.

Conclusion: The robotic approach failed to show a benefit in reducing postoperative pain or opioid requirements. Standardized multimodal therapy including routine TAP block demonstrated overall low pain scores and opioid requirements in both approaches.

\section{S124}

\section{MANAGEMENT OF CHOLEDOCHOLITHIASIS FOLLOWING ROUX-EN-Y GASTRIC BYPASS: A SYSTEMATIC REVIEW AND META-ANALYSIS}

Matthew Connell, MD; Warren Sun, MD; Jerry T Dang, MD; Valentin Mocanu, MD; Janice Y Kung, BCom, MLIS; Noah J Switzer, MD, MPH; Daniel W Birch, MD, MSc; Shahzeer Karmali, MD, MPH; University of Alberta.

INTRODUCTION: Choledocholithiasis is common in patients post Roux-en-Y gastric bypass (RYGB), however its post-surgical anatomy poses unique management challenges. Multiple alternatives to traditional endoscopic retrograde cholangio-pancreatography (ERCP) have been proposed, including laparoscopicassisted transgastric ERCP (LAERCP), endoscopic ultrasound (EUS)-directed transgastric ERCP (EDGE), balloon-assisted endoscopy (BAE), intra-gastric singleport ERCP (IGS ERCP), laparoscopic common bile duct exploration (LCBDE), and EUS-guided intra-hepatic puncture with antegrade clearance (EGHAC). The aim of this study was to conduct a systematic review evaluating the efficacy of these strategies in the management of choledocholithiasis post-RYGB.

METHODS: A comprehensive search in MEDLINE, EMBASE, Scopus, Web of Science, and Cochrane Library was performed on July 24, 2019 by a medical librarian. Two authors independently screened full-text articles. Observational and interventional studies reporting on the management of patients with choledocholithiasis post-RYGB including at least 5 patients were eligible for inclusion. Primary outcome was successful stone clearance. Secondary outcomes included procedure duration, length of hospital stay, and adverse events. Pooled rates of success were calculated for the primary outcome and pooled means were calculated for the secondary outcomes.

RESULTS: Of 2662 identified studies, 40 studies ( 31 retrospective cohort studies, 7 case series, and 2 prospective cohort studies) involving 566 patients were included in the final analysis. Included patients had a mean age of $52.6 \pm 6.24$ years, with 78.2 $\pm 14.2 \%$ female, and average BMI of $31.4 \pm 7.86 \mathrm{~kg} / \mathrm{m} 2$. Procedures represented in these studies included LAERCP $(\mathrm{n}=26$ studies, 381 patients), EDGE $(\mathrm{n}=5$ studies, 81 patients), LCBDE ( $\mathrm{n}=3$ studies, 37 patients), BAE ( $\mathrm{n}=3$ studies, 34 patients), IGS ERCP ( $\mathrm{n}=2$ studies, 14 patients), EGHAC ( $\mathrm{n}=2$ studies, 12 patients), and rendezvous guidewire-assisted ERCP ( $\mathrm{n}=1$ study, 7 patients). High rates of successful stone clearance were observed with LAERCP $(92.1 \%$; 95\% CI 88.6-94.6, p $<0.001)$, EDGE $(95.6 \% ; 95 \%$ CI $88.0-96.4, \mathrm{p}<0.001)$, IGS ERCP $(92.9 \%$; 95\% CI 66.3-99.1, $\mathrm{p}=0.009)$, and LCBDE $(96.1 \%$; 95\% CI 82.9-99.2, $\mathrm{p}<0.001)$. Lower rates of stone clearance were observed with $\mathrm{BAE}(61.5 \%$; 95\% CI 44.3-76.3, p = $0.188)$ and EGHAC $(74.0 \%$; 95\% CI 42.9-91.5, $\mathrm{p}=0.124)$. Relative to EDGE, LAERCP was associated with a longer procedure time (133.1 min vs. $67.4 \mathrm{~min}$ ) but lower complication rates $(12.8 \%$ vs. $24.3 \%)$.

CONCLUSIONS: LAERCP and EDGE have high rates of success in the management of choledocholithiasis post-RYGB. LAERCP has fewer complications but is associated with a longer procedure time. BAE has lower success rates than both LAERCP and EDGE. 
S125

\section{Morality and Legitimacy: Understanding Racially Diverse Perceptions of Bariatric Surgery}

Grace F Chao, MD, MS ${ }^{1}$; Mary E Byrnes, PhD, MUP²; Amir A Ghaferi, MD, $\mathrm{MS}^{3} ;{ }^{1}$ Veterans Affairs Ann Arbor; ${ }^{2}$ Center for Healthcare Outcomes and Policy; ${ }^{3}$ Michigan Bariatric Surgery Collaborative.

Background: There exists significant disparities in who receives bariatric surgery. Nationally, $70 \%$ of bariatric surgery patients are White women. However, Black and Hispanic populations are at higher risk for developing obesity. It is unclear why demographics of patients do not match the populations at risk for obesity. Previous studies have focused on physician, insurance, and medical factors that may impact the decision to undergo bariatric surgery. This study is the first qualitative study with a racially and ethnically diverse patient population to understand patient perceptions of bariatric surgery.

Methods: We interviewed 32 individuals who met NIH criteria for bariatric surgery but have never considered bariatric surgery. We purposively sampled to ensure the majority of participants were Black and/or Hispanic. An exploratory, iterative method was used to code transcribed interviews. We then employed an interpretive framework to arrive at our final themes.

Results: Participants self-identified as $88 \%$ female, $75 \%$ Black, 3\% Hispanic, 3\% Pacific Islander, and 19\% White. Three major themes emerged from our data. When examining responses, two themes were consistent across participants regardless of race/ethnicity. First, participants described being overweight as moral failure that should be resolved through discipline of the body. Participants' social context constructs being overweight as a visible sign of weakness, a personal inability to control appetite and diet. Thus, surgery was "cheating," contributing to its being considered one of last resort. Second, bariatric surgery was often not seen as component of mainstream medicine. It was viewed as offered at "places that may not be real hospitals" or something done in a foreign country. An important factor decreasing legitimacy for most participants was that their physicians had never mentioned bariatric surgery. Finally, among Black participants, fear of disfigurement was a major deterrent. These included concerns regarding excess skin, an unhealthy "hollowed out look," and being too small or "skinny skinny."

Conclusions: Though bariatric surgery is a safe, effective, and durable therapy for patients with obesity; the majority of individuals we interviewed face feelings of moral failure and concerns over the legitimacy of bariatric surgery. Thus, healthcare providers may be instrumental in 1) shifting from a discourse of moral failure towards one focused on health and 2) increasing education of providers and the public of bariatric surgery's role in obesity treatment. Healthcare systems may also be able to address post-operative concerns of Black individuals by including more Black bariatric surgery patient experiences in their public outreach efforts.

\section{S126}

\section{Marijuana use does not affect weight loss or complication rate} after bariatric surgery.

Tarin Worrest, MD; Jill Welshans, RN, MSN, AGACNP; Amanda Koonce, MD Elizabeth Dewey, MS; Farah Husain, MD; Oregon Health and Science University.

INTRODUCTION: Marijuana use has become more accepted as it has been legalized in several states. Although marijuana use has been linked to increased appetite due to effects on the cannabinoid receptors, it is unclear if use affects weight loss outcomes or complication rates following bariatric surgery. The purpose of this study was to determine if patients who use marijuana had higher complication rates or lower weight loss compared with non-users.

METHODS: All patients who underwent primary bariatric surgery (Roux-enYgastric bypass or sleeve gastrectomy) between July 2015 and July 2020 at a single institution after the legalization of marijuana within the state. All patients were asked about current or prior marijuana use as part of their referral to our bariatric center. Demographics, weights over time, complications, and marijuana use were abstracted from patient charts in a retrospective fashion. Differences between groups were evaluated with Wilcoxon Rank-Sum tests and Fisher Freeman Halton test. Trends for marijuana use over time was evaluated with simple linear regression on summary data. Significance was assessed at $\mathrm{p}<0.05$, and analysis was conducted in JMP 15.2 and SAS 9.4 (SAS Inc., Cary, NC).

RESULTS: 1107 patients were included within the study. $798(73.3 \%)$ were never users (NU), $225(19.4 \%)$ were previous users (PU), and 84 (7.2\%) were active users (AU). The proportion of AU and PU increased over time, with significantly more prior marijuana use reported in more recent years $(p=0.014)$ (Fig. 1). AU had significantly higher pre-procedural BMIs than NU: 48.7 vs. $46.3(\mathrm{p}=0.03)$. Any marijuana use (AU and PU) was associated with higher preoperative weight compared to NU: $136.4 \mathrm{~kg}$ vs. $130.6 \mathrm{~kg}(\mathrm{p}=0.001)$. Overall complication rate was low in all groups, and there was no difference in the rates of any complications. AU and PU tended to lose less weight than NU, but this was not statistically significant ( $\mathrm{p}=$ $0.17)$

CONCLUSIONS: Active and prior marijuana users tend to have higher BMIs on presentation to our bariatric surgery program, but use is not associated with complications or percent weight loss. The incidence of patient reported marijuana use is increasing in our bariatric surgery population. More studies on the effects of marijuana use in this patient population are warranted.

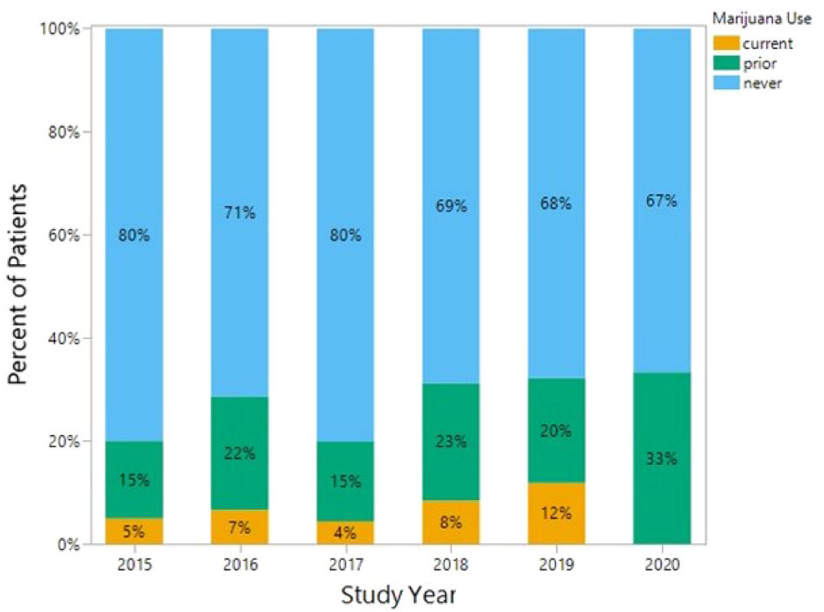

Figure 1: Patient reported Marijuana use over time. Patients reporting prior use increased over the study period $(p=0.014)$. 
S127

Is staple line oversewing in patients on chronic anticoagulation during laparoscopic sleeve gastrectomy necessary? Propensity Score Matching Analysis using the 2015-2018 MBSAQIP

Raul Sebastian, $\mathrm{MD}^{1}$; Jorge Cornejo, $\mathrm{MD}^{1}$; Omar Ghanem, $\mathrm{MD}^{2}$; Thomas Ruttger ${ }^{1}$; Christina Li, MD ${ }^{1}{ }^{1}$ Northwest Hospital; ${ }^{2}$ Mayo Clinic.

Introduction: There is an increased incidence of postoperative bleeding in patients on chronic anticoagulation (CAC), therefore some surgeons propose oversewing the sleeve staple line as a preventive measure. However, there is no clear evidence to assess the effectiveness of oversewing the staple line during laparoscopic sleeve gastrectomy (LSG) in bariatric patients on CAC.

Methods: Using the 2015-2018 Metabolic and Bariatric Surgery Accreditation and Quality Improvement Program (MBSAQIP) database, patients between 18- and 65 -years old who underwent LSG were included. First, to investigate the role of CAC, we performed 1:1 Propensity-Score Matching (PSM) between the CAC and non-CAC patients. Second, to explore the impact of oversewing we focused only on the CAC patients and divided in 2 groups, the oversewing and non-oversewing and 1:1 PSM was performed also. To avoid potential confounders, the PSM analyses in both groups were performed using 22 preoperative characteristics. Then 30-day postoperative outcomes including bleeding and blood transfusion requirements were assessed.

Results: 402,826 patients underwent LSG, $2.3 \%(\mathrm{n}=9148)$ were on CAC. In the CAC cohort (8843 matched cases), the anticoagulated patients showed a significant increase in postoperative bleeding $(1.2 \%$ vs. $0.5 \% ; \mathrm{P}<0.001)$, blood transfusion requirements $(1.7 \%$ vs. $0.7 \%$; $\mathrm{P}<0.001)$, unplanned ICU admissions $(2.0 \%$ vs. $1,3 \% ; \mathrm{P}=0.001)$, interventions $(2.0 \%$ vs. $1.5 \% ; \mathrm{P}=0.015)$ and readmissions $(6.2 \%$ vs. $4.7 \%$; $\mathrm{P}<0.001) .1939(21.2 \%)$ patients on $\mathrm{CAC}$ underwent oversewing during the LSG. The operative time was significantly longer in these patients $(87.11 \pm 40$ vs. $76.19 \pm 37 ; \mathrm{P}<0.001)$. Patients that underwent oversewing showed similar results in 30-day outcomes as those that did not, with no statistical difference including postoperative bleeding $(1.0 \%$ vs. $0.9 \% ; \mathrm{P}=0.8)$ and blood transfusion requirements $(1.4 \%$ vs $1.8 \% ; \mathrm{P}=00.9)$.

Conclusion: Incidence of post-operative bleeding and blood transfusion requirements are higher in patients on CAC during the LSG, oversewing can be performed or omitted without compromising 30-day post-operative outcomes, despite longer operative times in patients on CAC

\section{S128}

\section{ASSESSING RISK OF NARCOTIC OVERDOSE IN PATIENTS UNDERGOING BARIATRIC SURGERY}

Phillip Yang ${ }^{1}$; Aaron J Bonham ${ }^{2}$; Arthur M Carlin ${ }^{3}$; Jonathan F Finks ${ }^{2}$; Amir A Ghaferi ${ }^{2}$; Oliver A Varban ${ }^{2} ;{ }^{1}$ University of Michigan Medical School; ${ }^{2}$ Department of Surgery, Michigan Medicine; ${ }^{3}$ Department of Surgery, Henry Ford Health System.

Introduction: Obesity-related chronic pain can increase the risk of narcotic abuse in bariatric surgery patients. However, assessment of overdose risk has not been evaluated to date.

Methods: A NARxCHECK ${ }^{\circledR}$ overdose score (i.e. "Narx score") was calculated preoperatively on all patients undergoing bariatric surgery $(n=306)$ between 2018 and 2020 at a single center academic bariatric surgery program. The 3-digit score ranges from low risk (000) to high risk (999) and is based on patient risk factors found within the Prescription Drug Monitoring Program. Patient characteristics, comorbidities, length of stay and emergency room (ER) visits were compared between patients with high (score $>200$ ) and low risk (score $=000)$ for overdose Morphine milligram equivalent (MME) prescribed at discharge and refills were also evaluated.

Results: Patients with a Narx score $>200$ represented $28 \%(n=85)$ of all patients undergoing bariatric surgery and compared to the lowest risk group $(\mathrm{n}=101,33 \%)$, were more likely to have depression $(60.0 \%$ vs $38.6 \%, \mathrm{p}=0.005)$, anxiety $(45.9 \%$ vs $30.7 \%, \mathrm{p}=0.0351)$ and bipolar disorder $(7.1 \%$ vs $0.0 \%, \mathrm{p}=0.0083)$. Median MME prescribed at discharge was the same between high and low risk groups (75), however, high risk patients were more likely to be prescribed more than 10 tablets of a secondary opioid $(83.3 \%$ vs $0 \%, p=0.0111)$, which was prescribed by another provider in $66.7 \%$ of the cases. ER visits among patients who did not have a complication or require a readmission was also higher among high risk patients $(8.8 \%$ vs $0 \%, p=0.0083)$. There were no deaths or incidents of mental health related visits to the ER in either group.

Conclusions: Over a quarter of patients undergoing bariatric surgery have an increased risk of narcotic overdose. High risk patients are more likely to have mental health disorders and are also more likely to present to the ER unnecessarily in the setting of standardized opioid prescribing practices. Narx scores can help reduce ER visits by identifying at-risk patients who may benefit from additional clinic or telehealth follow-up. 


\section{S129}

\section{A MODULAR SIMULATION CURRICULUM TO TEACH ENDOSCOPIC STENTING TO PRACTICING SURGEONS: AN “INTO THE FIRE” APPROACH}

Harry J Wong, $\mathrm{MD}^{1}$; Mikhail Attaar, $\mathrm{MD}^{1}$; Hoover $\mathrm{Wu}, \mathrm{MD}^{1}$; Michelle Campbell, $\mathrm{MD}^{1}$; Kristine Kuchta, $\mathrm{MS}^{2}$; Stephen Haggerty, MD, FACS ${ }^{2}$; John Linn, MD, FACS $^{2}$; Woody Denham, MD, FACS ${ }^{2}$; Michael Ujiki, MD, FACS ${ }^{2} ;{ }^{1}$ Northshore University HealthSystem; University of Chicago Medicine; ${ }^{2}$ Northshore University HealthSystem

Introduction: Flexible endoscopy is a valuable tool for the gastrointestinal (GI) surgeon and can provide minimally invasive therapy for surgical problems. There is a need for effective and efficient training curricula in therapeutic endoscopic techniques for trainees and practicing providers. Here, we present a simulation-based modular curriculum using an "into the fire" approach with hands-on pre- and posttesting to teach endoscopic stenting to practicing physicians.

Methods: Two advanced flexible endoscopy courses were taught by expert surgical endoscopists from 2018 to 2019. The stenting module involved using self-expandable metal stents to manage simulated esophageal and gastroduodenal strictures on a non-tissue GI model. Based on the educational theories of inquiry-based learning and guided discovery learning, the simulation curriculum was designed with a series of pre-training tests, didactics, mentored hands-on instructions, and post-training tests. Both pre- and post-testing included a confidence survey, knowledge-based written test, and evaluation form specific to the hands-on performance of the stenting techniques. Wilcoxon signed-rank tests were used to compare pre- and post-tests scores

Results: Twenty-eight practicing physicians with varying endoscopic experiences participated in the course. The majority of the participants $(67.9 \%)$ had completed over 100 upper endoscopic procedures and $57.1 \%$ were certified in Fundamentals of Endoscopic Surgery. After completing the modular curriculum, participant confidence survey scores improved from $11.4 \pm 4.2$ to $20.7 \pm 4.0(\mathrm{p}<0.001)$ Knowledge-based written test scores also improved from $7.1 \pm 1.2$ to $8.4 \pm 0.9$ (p $<$ $0.001)$. In terms of technical performance, overall hands-on performance scores improved from $21.3 \pm 2.7$ to $28.9 \pm 1.2(\mathrm{p}<0.001)$ with significant improvement in each individual component of the assessment (all $\mathrm{p}$ values $<0.01$ ), and the greatest improvement seen in equipment handling (88\%) and flow of procedure (54\%).

Conclusion: Our modular simulation curriculum using an "into the fire" approach to teach endoscopic stenting is effective in improving learner knowledge, confidence and hands-on performance of technical skills. By starting the training with a handson pre-test, our approach allows learners to explore the equipment and technique before an expert-guided discovery learning process, which is both effective, efficient, and adaptable to teaching practicing physicians with varying levels of experience.

\section{S130}

\section{CONSENSUS PRIORITY RESEARCH QUESTIONS IN GASTROINTESTINAL AND ENDOSCOPIC SURGERY IN THE YEAR 2020: RESULTS OF A SAGES DELPHI STUDY}

Richard Garfinkle, MD, $\mathrm{MSc}^{1}$; Boris Zevin, MD, $\mathrm{PhD}^{2}$; Chris DuCoin, $\mathrm{MD}, \mathrm{MPH}^{3}$; Maria S Altieri, MD, $\mathrm{MS}^{4}$; Rajesh Aggarwal, $\mathrm{MD}, \mathrm{PhD}^{5}$; Aurora Pryor, $\mathrm{MD}^{6}$; Rebecca P Petersen, MD, $\mathrm{MSc}^{7} ;{ }^{1}$ Department of Surgery, McGill University, Montreal, QC, Canada; ${ }^{2}$ Department of Surgery, Queen's University, Kingston, ON, Canada; ${ }^{3}$ Division of Gastrointestinal Surgery, University of South Florida, Tampa Bay, FLA, USA; ${ }^{4}$ Department of Surgery, East Carolina University Brody School of Medicine, Greenville, NC, USA; ${ }^{5}$ Department of Surgery, Thomas Jefferson University, Philadelphia, PA, USA; ' ${ }^{6}$ Department of Surgery, Stony Brook Medicine, New York, NY, USA; ${ }^{7}$ Department of Surgery, University of Washington, Seattle, WA, USA.

INTRODUCTION: Given the rapid evolution in gastrointestinal and endoscopic surgery, the identification of important research topics could help concentrate efforts on high-yield endeavours. The objectives of this study were to identify consensus priority research questions according to members of the Society of American Gastrointestinal and Endoscopic Surgeons (SAGES), and to study differences in priorities according to specific membership subgroups.
METHODS AND PROCEDURES: An initial list of research questions was compiled by members of 26 SAGES Committees and Task Forces, and was refined using a nominal group technique by the Delphi Task Force. The questions were divided into five categories: (1) Surgical Outcomes; (2) Education, Training, and Simulation; (3) Health Services Research; (4) New Technology; and (5) Artificial Intelligence. The online survey was distributed to the SAGES membership via email and social media platforms for three consecutive rounds of ranking. Respondents were asked to rank each question with regards to its importance in the field of gastrointestinal and endoscopic surgery (1 - low; 5 - high). "Priority" was defined as a single-round mean score of $\geq 3.5$, and "consensus" as a single-round standard deviation $<1.0$. The Delphi was terminated for any single category after 5 consensus priority questions were achieved, or after a maximum of three rounds. Subgroup analyses were performed according to a priori selected respondent characteristics. RESULTS: Rounds 1, 2 and 3 were disseminated in April, June, and August 2020, respectively. The total number of respondents for each round was: Round $1(\mathrm{n}=$ 407); Round $2(\mathrm{n}=569)$; Round $3(\mathrm{n}=273)$. In each round, the majority of respondents were male (Round 1: 77.4\%; Round 2: 77.1\%; Round 3: 76.7\%), selfidentified as academic (vs. community) surgeons (Round 1: $57.1 \%$; Round 2: $61.1 \%$; Round 3: 60.2\%), and practiced in North America (Round 1: 71.8\%; Round 2: 70.8\%; Round 3: $75.9 \%$ ). A total of 29 out of 122 questions achieved consensus priority (Fig. 1). "Surgical Outcomes" was the only research category to achieve more than 5 consensus priority questions in Round 1, and was omitted from subsequent rounds. Differences in scores for consensus priority questions were reported among several respondent subgroups, including sex (male vs. female), years in independent practice $(<10$ vs. $\geq 10)$, and type of practice (academic vs. community).

CONCLUSION(S): Consensus priority research questions in gastrointestinal and endoscopic surgery were identified across five different research categories. These results can provide direction and areas of interest for funding and investigation for future studies.

Figure 1 - Consensus priority questions by research category

\begin{tabular}{|c|c|}
\hline Research Question & $\begin{array}{l}\text { Mean score } \\
\text { (SD) }\end{array}$ \\
\hline \multicolumn{2}{|l|}{ Surgical Outcomes } \\
\hline 1. How do modifiable risk factors (obesity, tobacco abuse, etc.) affect surgical outcomes? & $4.17(0.96)$ \\
\hline 2. What is the optimal treatment for obesity? & $4.01(0.99)$ \\
\hline $\begin{array}{l}\text { 3. What is the most effective treatment option for: a) GERD alone; b) hiatal hernia with } \\
\text { GERD; c) esophageal dysmotility with GERD and or hiatal hernia? }\end{array}$ & $3.94(0.96)$ \\
\hline 4. What is the optimal education tool to engage patients in their care? & $3.82(0.99)$ \\
\hline 5. Does reporting surgeon outcomes have a measurable effect on patient outcomes? & $3.71(0.99)$ \\
\hline 6. How are risk prediction tools currently used in surgical practice? & $3.50(0.97)$ \\
\hline \multicolumn{2}{|l|}{ Education, Training, and Simulation } \\
\hline 7. How can we best utilize video-based education and coaching to adopt new techniques? & $4.11(0.83)$ \\
\hline $\begin{array}{l}\text { 8. What is the best method to teach newer techniquestechnology to surgeons already in } \\
\text { practice (including those in international, low resource, or community settings)? }\end{array}$ & $3.90(0.99)$ \\
\hline 9. What is the impact of video-based assessment on the surgeon's clinical outcomes? & 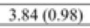 \\
\hline 10. How effective are hands-on courses in transfer to practice? & $3.81(0.98)$ \\
\hline $\begin{array}{l}\text { 11. How much training is needed to aequire proficiency in advanced techniques (e.g, flexible } \\
\text { endoscopy, robotics)? }\end{array}$ & $3.80(0.98)$ \\
\hline 12. What are the advanced endoscopic skills required of general surgeons? & $3.76(0.99)$ \\
\hline $\begin{array}{l}\text { 13. How can we utilize courses and webinars to disseminate new technologies to residency } \\
\text { programs? }\end{array}$ & $3.69(0.99)$ \\
\hline $\begin{array}{l}\text { 14. Can competency in laparoseopic and robotic simulation be aceurately correlated with } \\
\text { operative autonomy and competency in patients? }\end{array}$ & $3.60(0$. \\
\hline $\begin{array}{l}\text { 15. How can we effectively teach and evaluate new technologies through current SAGES } \\
\text { mechanisms (ADOPT, Master's Program, etc.)? }\end{array}$ & $3.52(0.94)$ \\
\hline \multicolumn{2}{|l|}{ Health Services Research } \\
\hline 16. Which elements of enhanced recovery pathways $\mathrm{h}$ & $4.12(0.93)$ \\
\hline $\begin{array}{l}\text { 17. Does enhanced recovery after surgery benefit all patient populations or are there outliers } \\
\text { where there is decreased benefit or harm? }\end{array}$ & $3.88(0.95)$ \\
\hline $\begin{array}{l}\text { 18. Do standardized prehabilitation programs improve outcomes of elective surgical } \\
\text { procedures, and which patients benefit the most? }\end{array}$ & $3.87(0.93)$ \\
\hline 19. How does centralizing care in specialized centers affect patient access to care? & $3.84(0.95)$ \\
\hline 20. What is the relationship between surgical innovation and health care quality and costs? & $3.78(0.93)$ \\
\hline \multicolumn{2}{|l|}{ New Technology } \\
\hline 21 . How can we decrease the rate of staple line leaks wi & $4.20(0.85)$ \\
\hline 22. What outeomes are important to measure after the implementation of new technology? & $4.05(0.84)$ \\
\hline $\begin{array}{l}\text { 23. During cholecystectomy, do visualization techniques (near infra-red cholangiography, } \\
\text { white light and IOC) prevent bile duct injury? }\end{array}$ & $3.78(0.99)$ \\
\hline $\begin{array}{l}\text { 24. Should there be standards of interoperability (set by industry or physician groups) for new } \\
\text { surgical devices, platforms, artificial intelligence, and data collection programs? }\end{array}$ & $3.77(0.99)$ \\
\hline 25. What are the clinical advantages of near-infrared fluorescence in laparoscopy? & $3.60(0.97)$ \\
\hline \multicolumn{2}{|l|}{ Artificial Intelligence } \\
\hline $\begin{array}{l}\text { 26. How can artificial intelligence be used to help with preoperative and intraoperative } \\
\text { decision making for patient safety? }\end{array}$ & $3.85(0.87)$ \\
\hline 27. How can artificial intelligence be used in training and education to improve competency? & $3.79(0.95)$ \\
\hline 28. Can artificial intelligence assist in recognition of pathology in endoscopy? & $3.78(0.99)$ \\
\hline $\begin{array}{l}\text { 29. Can an algorithm be developed to support decision making regarding the management of } \\
\text { high-risk colorectal anastomoses? }\end{array}$ & 6) \\
\hline
\end{tabular}




\section{S131}

\section{Management of the colonic polyps referred for surgery: an opportunity for improvement}

Cecily Stockley, MD, $\mathrm{MPH}^{1}$; Bradley Evans, MD, $\mathrm{MSc}^{2}$; Mark R Borgaonkar, MD, MSc, FRCPC ${ }^{1}$; Muna Lougheed, $\mathrm{MD}^{1}$; Haley Flemming, $\mathrm{MD}^{1}$; Altaf Taher, MDPath, FRCPC, FABP ${ }^{1}$; David Pace, MD, FRCSC ${ }^{1} ;{ }^{1}$ Memorial University of Newfoundland; ${ }^{2}$ Dalhousie University.

Purpose: To understand the decision-making process of endoscopists when referring patients with colonic polyps for surgery and determine the surgical morbidity and mortality associated with these decisions.

Methods: This retrospective cohort study was conducted on patients who underwent colonic resection from January 1, 2014 to July 18, 2019 in our regional health authority. 2500 resections were in the database; 250 cases were identified using the search term "polyp" or "adenoma". Charts were then reviewed using exclusion criteria: cancer diagnosis, emergency surgery, more than one colonic resection, and subtotal colectomy for polyposis. The primary outcome was surgical mortality. Secondary outcomes included surgical morbidity and the decision-making strategies used to determine unresectability.

Results: A total of 106 cases fulfilled eligibility criteria and were included in the study. Mean patient age was 65.3 years $( \pm 8.6$ years) and there were more males (n $=64)$ than females $(n=42)$. Factors used by endoscopists to determine unresectability included polyp size, morphology, and location. Mean polyp size was $32.7 \mathrm{~mm}( \pm 19.5 \mathrm{~mm}) .30$ patients $(28.6 \%)$ had polyps equal to or less than $2 \mathrm{~cm}$. While the vast majority of polyps described were sessile, 13 patients $(12.3 \%)$ had pedunculated polyps and morphology was not described for 15 patients $(14.2 \%)$. Polyps were predominantly located in the cecum $(n=44)$, the right colon $(n=25)$, and at the hepatic flexure $(\mathrm{n}=15)$. An attempt at colonoscopic resection was made in 31 patients $(29.2 \%)$ and five cases $(4.7 \%)$ were referred for a second opinion regarding endoscopic resection. Endoscopists incorrectly felt that polyps were malignant in 62 cases $(58.5 \%)$.

At the time of surgery, the majority of patients had an ASA classification of II $(\mathrm{n}=$ $64,60.4 \%)$ or III $(\mathrm{n}=26,24.5 \%)$. Using the Clavien-Dindo classification of postoperative complications, the majority of patients had either no complications $n=36$ $(34.0 \%)$ or Grade I complications $(\mathrm{n}=41,38.7 \%)$. Twelve patients $(11.3 \%)$ had Grade II complications including infections requiring antibiotics, blood transfusions, or total parental nutrition. Nine patients $(8.5 \%)$ had Grade III complications requiring surgical or endoscopic management. Six patients $(5.7 \%)$ had Grade IV complications requiring ICU admission and two patients (1.9\%) died. Both deaths were in ASA III class patients (moderate-severe systemic disease).

Conclusion: Surgery for benign colonic polyps is associated with significant morbidity and mortality. A minority of patients had an attempt at endoscopic resection and only $4.7 \%$ were referred for a second endoscopic opinion. These findings reveal a gap in endoscopic management of benign colonic polyps.

\section{S132}

\section{A MODULAR SIMULATION CURRICULUM TO TEACH ENDOSCOPIC DILATION TO PRACTICING SURGEONS: AN "INTO THE FIRE" APPROACH}

Harry J Wong, MD ${ }^{1}$; Mikhail Attaar, $\mathrm{MD}^{1}$; Michelle Campbell, $\mathrm{MD}^{1}$; Hoover Wu, $\overline{M D}^{1}$; Kristine Kuchta, $\mathrm{MS}^{2}$; John Linn, MD, FACS ${ }^{2}$; Stephen Haggerty, MD, FACS ${ }^{2}$; Woody Denham, MD, FACS ${ }^{2}$; Michael Ujiki, MD, FACS ${ }^{2} ;{ }^{1}$ Northshore University HealthSystem; University of Chicago Medicine; ${ }^{2}$ Northshore University HealthSystem.

Introduction: With the ability to manage surgical problems minimally invasively, flexible endoscopy has become a vital tool for gastrointestinal (GI) surgeons. However, limited resources exist on effective and efficient training methods for the practicing surgeon. Here, we present a simulation-based modular curriculum using an "into the fire" approach with hands-on pre- and post-testing to teach endoscopic dilation to practicing physicians.

Methods: From 2018 to 2019, two advanced flexible endoscopy courses with multiple modules were taught by practicing gastroenterologists and surgeons. The dilation module focuses on using a through-the-scope balloon dilation technique to dilate simulated esophageal strictures, using ex-vivo porcine models. Using the principles of inquiry-based learning and guided discovery learning, the simulation curriculum is composed of a series of pre-training tests, didactic and mentored hands-on instructions, and post-training tests. Assessment tools included a knowledge-based written test, a confidence survey, and evaluation form specific to the hands-on performance of the dilation technique. Pre- and post-test scores were compared using Wilcoxon signed-rank test.

Results: Out of 28 practicing physicians who completed the course, the majority $(69.7 \%)$ had performed over 100 upper endoscopic procedures and $57.1 \%$ were certified in Fundamentals of Endoscopic Surgery. After completion of the simulation, participants had improved written test scores from $7.1 \pm 1.2$ to $8.4 \pm 0.9$ (p $<$ $0.001)$ and confidence survey scores from $11.4 \pm 4.2$ to $20.7 \pm 4.0(\mathrm{p}<0.001)$ Technical hands-on performance score of endoscopic dilation improved from $22.5 \pm$ 2.8 to $30.0 \pm 0.0(\mathrm{p}<0.001)$. There were significant improvements in all components of the hands-on skill with the greatest improvement noted in knowledge of procedure $(56 \%)$ and appropriate balloon placement (138\%).

Conclusion: Using an "into the fire" approach, the dilation module of our flexible endoscopy simulation curriculum is effective in improving participants' knowledge, confidence and hands-on performance of the technical skills in endoscopic stenting. This unique approach of using a hands-on pre-test helps create a more learnercentered environment and addresses the unique challenges of teaching practicing physicians with varying levels of experience. 
S133

\section{Clean Versus Sterile Operative Field During Percutaneous Endoscopic Gastrostomy: A Cost-Effectiveness Analysis}

David J Morrell; Alexander T Liu; Vamsi V Alli; Eric M Pauli; Joshua S Winder; Penn State Health Milton S. Hershey Medical Center.

Introduction Despite the non-sterile nature of the alimentary tract, percutaneous endoscopic gastrostomy (PEG) insertion is often done under sterile operative conditions to reduce infection risk. Our group has previously demonstrated no difference in the rate of surgical site infection (SSI) following PEG performed with clean or sterile operative fields. The purpose of this study is to evaluate the cost-effectiveness of clean versus sterile operative fields during PEG.

Methods A decision tree model of clean versus sterile operative fields during PEG was created and model parameters were obtained from retrospective review of institutional cost and surgical outcomes databases. Additional model parameters were obtained through literature review. The primary outcome was incremental costeffectiveness ratio (ICER). Costs were estimated from the perspective of the healthcare system provider with the most cost-effective strategy defined as an ICER of $\$ 100,000 /$ quality-adjusted life year (QALY) or less.

Results From the institutional database, 247 patients undergoing clean operative field PEG and 208 patients undergoing sterile operative field PEG were identified with SSI rates of $2.02 \%$ and $1.44 \%$ respectively. In the base case analysis, using our institutional SSI rate, the marginal cost of sterile operative field PEG was $\$ 536.10$ more costly and the marginal effectiveness was 0.00003 QALY resulting in an ICER of $\$ 15,403,937$. On one-way sensitivity analysis holding all other variables constant, clean operative field PEG remained the most cost effective when the cost was $\$ 124$ less than that of sterile operative field PEG or when the SSI rate did not exceed $4.52 \%$. Two-way sensitivity analysis was used to demonstrate whether ICER was sensitive to differences in the rate of SSI after clean and sterile operative field PEG. Clean operative field PEGs remained cost effective when the SSI rate after clean operative field procedures did not exceed $3 \%$ relative to the SSI rate after sterile operative field procedures.

Conclusions PEG procedures performed with a clean operative field are cost effective compared to procedures performed with a sterile operative field. Potential increases in SSI rates that may accompany foregoing a sterile operative field do not offset the cost savings of a clean operative field.

\section{S134}

\section{A PROSPECTIVE CASE SERIES OF ONE HUNDRED TWENTY-SEVEN PATIENTS WITH PERORAL ENDOSCOPIC MYOTOMY (POEM)}

Aman Ali, MD; Flavio Baio, MD; Alfred Lopez, MD; Mahnoor Zia; Lee Morris, MD, FACS; Houston Methodist Hospital.

Background: Achalasia is a very rare disorder with an annual incidence of $1 / 100,000$ people and a prevalence of $10 / 100,000$ in the United States. Perora endoscopic myotomy (POEM), initially described by Dr. Inoue, has been adopted by some as a less invasive alternative to laparoscopic Heller myotomy. Our goal was to evaluate the safety and efficacy of POEM for the treatment of achalasia.

Methods: We performed a single center retrospective review of data collected from patients who had undergone POEM for the treatment of achalasia. The study consisted of 127 patients between September 2013 to August 2020. Patient demographics, procedure details, previous treatment, and postoperative complications were recorded. To assess efficacy, preoperative and postoperative Dysphagia Handicap Index (DHI), and Quality of Life in Reflux and Dyspepsia (QOLRAD) scores were obtained on 78 patients.

Results: Table 1 reports patient demographics, types of achalasia, and previous treatments. Data suggested that $35.4 \%(n=45 / 127)$ of patients had previous procedural intervention for achalasia. Despite relapse of symptoms from prior interventions, POEM was completed in all patients without the need for conversion to laparoscopic Heller myotomy or open surgery. Average preoperative maximum esophageal width was $3.55[2.60-4.50] \mathrm{cm}$. Intra-operative complications were noted in $33.1 \%(n=42 / 120)$ of the patients, these included pneumoperitoneum and unintended mucosotomy. The postoperative DHI scores dropped significantly (58.4 preop and 17.7 postop; $\mathrm{p}$ value $<0.001$ ) and there was improvement in the average QOLRAD scores (4.4 preop and 6.6 postop; $p$ value $<0.001)$ - representing no significant development of gastroesophageal reflux after POEM. Postoperative narcotics were used by only $22.5 \%$ of the patients, and average length of hospital stay was 1-day.

Conclusion: Overall complication rates and postoperative narcotic use were low when compared to literature on laparoscopic Heller myotomy. This case series suggests that POEM can safely be performed in patients with achalasia.

Table 1. Patient Demographics and Outcomes ( $n=127$ )

\begin{tabular}{|c|c|}
\hline Patent Characteristics, $\mathrm{n}=\mathbf{1 2 7}$ & Data \\
\hline Age, y, mean (range) & $57.3 \pm 15.7$ \\
\hline Male $(n, \%)$ & $65(51.6 \%)$ \\
\hline Duration of symptoms, years, mean (range) & $6(0.5-40)$ \\
\hline Narcotic Use (total \# patients with narcotics s/p POEM/total \# of POEM patients, \%) & $\begin{array}{c}18 / 80 \\
(22.5 \%) \\
\end{array}$ \\
\hline Length of hospital stay after POEM, days; median (range) & $1(1-8)$ \\
\hline \multicolumn{2}{|l|}{ Chicago Classifications, $n=94$} \\
\hline Type I & 24 \\
\hline Type II & 49 \\
\hline Type III & 21 \\
\hline \multicolumn{2}{|l|}{ Previous Treatment, $\mathrm{n}=127$} \\
\hline Pneumatic dilation & 31 \\
\hline Botox injection & 21 \\
\hline Heller myotomy & 8 \\
\hline Prior GEJ Procedure & 45 \\
\hline No prior treatment & 82 \\
\hline
\end{tabular}




\section{S135}

\section{Geographic Distribution of Colonoscopy Providers in the United States: An Analysis of Medicare Claims Data}

Heather Carmichael, MD ${ }^{1}$; Jason M Samuels, $\mathrm{MD}^{1}$; Catherine G Velopulos, MD, $\mathrm{MHS}^{1}$; Edward L Jones, $\mathrm{MD}^{2} ;{ }^{1}$ University of Colorado; ${ }^{2}$ University of Colorado Rocky Mountain Veterans' Affairs Medical Center.

Introduction: Screening colonoscopy is one of the few procedures that can prevent cancer. The American Board of Surgery recently required objective testing of graduating chief surgical residents via the SAGES Fundamentals of Endoscopic Surgery curriculum in order to ensure widespread, high-quality access to endoscopy. While the majority of screening colonoscopy is performed by gastroenterologists, general surgeons likely play a key role in at-risk, rural populations. The aim of this study was to examine geographic practice patterns in screening colonoscopy using a nationwide claims database. Our hypothesis is that surgeons perform a majority of screening colonoscopies in non-urban areas.

Methods and Procedures: The 2017 Medicare Provider Utilization and Payment database was used to identify providers performing screening colonoscopy. Providers performing less than 10 procedures are not included in the dataset; the remaining "high volume" providers were classified as gastroenterologists, surgeons, ambulatory surgical centers (ASCs) or other providers. Rural-Urban Commuting Area classification at the zip code level was used to determine whether the practice location for an individual provider was in a rural area/small town $(<10,000$ people $)$, micropolitan area (10-50,000 people), or metropolitan area ( $>50,000$ people).

Results: Claims data from 765,666 screening colonoscopy procedures on Medicare patients were included; of these, 677,902 were performed by high-volume providers $(88.5 \%)$. The majority of procedures were performed by gastroenterologists $(49.0 \%)$ and ASCs (37.5\%). Surgeons performed $9.7 \%$ of cases overall. However, in some states, surgeons performed more than a third $(35 \%)$ of all included procedures (Fig. 1). When examined at a zip code level, surgeons performed $56.6 \%$ of procedures in small towns/rural areas and $27.1 \%$ of procedures in micropolitan areas. Individual surgeons performed fewer annual procedures as compared to gastroenterologists (median 26 vs. $39, \mathrm{p}<0.001$ ).

Conclusions: Surgeons perform the majority of screening colonoscopies in rural zip codes on Medicare patients. High quality, surgical training in endoscopy is essential to ensure access to colonoscopy for patients outside of major metropolitan areas.

\begin{tabular}{lllll}
\hline & Gastroenterologists & Surgeons & ASC & Other \\
\hline $\begin{array}{l}\text { Total } \\
\text { procedures }\end{array}$ & $330,641(49.0 \%)$ & $65,421(9.7 \%)$ & $252,777(37.5 \%)$ & $25,904(3.8 \%)$ \\
\hline Total providers & $6,759(61.9 \%)$ & $1,897(17.4 \%)$ & $1,581(14.5 \%)$ & $675(6.2 \%)$ \\
\hline $\begin{array}{l}\text { Median } \\
\text { procedures per } \\
\text { provider [IQR] }\end{array}$ & $39[20-64]$ & $26[16-43]$ & $117[53-221]$ & $26[15-48]$ \\
\hline $\begin{array}{l}\text { Metropolitan } \\
\text { area }\end{array}$ & $294,641(51.1 \%)$ & $33,968(5.9 \%)$ & $231,455(40.1 \%)$ & $16,922(2.9 \%)$ \\
\hline $\begin{array}{l}\text { Micropolitan } \\
\text { areas }\end{array}$ & $32,742(40.5 \%)$ & $21,930(27.1 \%)$ & $20,397(25.2 \%)$ & $5,872(7.3 \%)$ \\
\hline $\begin{array}{l}\text { Small town or } \\
\text { rural area }\end{array}$ & $3,258(19.4 \%)$ & $9,523(56.6 \%)$ & $925(5.5 \%)$ & $3,110(18.5 \%)$ \\
\hline
\end{tabular}

Percent of Screening Colonoscopies Performed by Surgeons

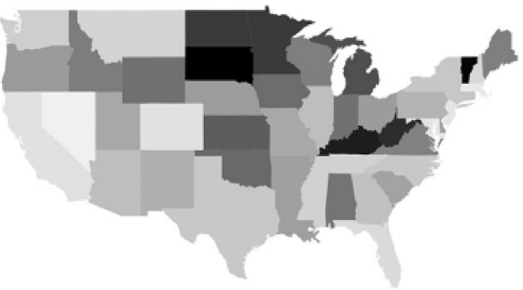

\section{S136}

Does your team know how to respond safely to an operating room fire? Outcomes of a virtual reality, AI-enhanced simulation training

Hung P Truong, MD, MS ${ }^{1}$; Di Qi, $\mathrm{PhD}^{2}$; Adam Ryason, PhD, BS ${ }^{2}$; Amy M Sullivan, $\mathrm{EdD}^{3}$; Jaime Cudmore'; Samuel Alfred, $\mathrm{PhD}^{2}$; Stephanie B Jones, $\mathrm{MD}^{4}$; Jose $\mathrm{M}$ Parra, MPH, BA ${ }^{3}$; Suvranu De, Sc, $\mathrm{D}^{2}$; Daniel B Jones, MD, MS ${ }^{1} ;{ }^{1}$ Department of Surgery, Beth Israel Deaconess Medical Center, Harvard School of Medicine; ${ }^{2}$ Center for Modeling, Simulation, and Imaging in Medicine, Rensselaer Polytechnic Institute; ${ }^{3} \mathrm{Carl}$ J. Shapiro Institute for Research and Education, Beth Israel Deaconess Medical Center, Harvard Medical School; ${ }^{4}$ Department of Anesthesia and Critical Care, Albany Medical Center.

Introduction: Operating room (OR) fires are rare but devastating events requiring immediate and effective response. Virtual Reality (VR) simulation training can provide a safe environment for the deliberate practice of skills in such highly stressful situation. This study assessed interprofessional participants' ability to respond to VR simulated OR fire scenarios, attitudes, numbers of attempt of the VR simulation do participants need to successfully respond to OR fires and does prior experience, confidence level, or professional role predict the number of attempts needed to demonstrate safety and pass the simulation.

Methods: 180 surgical team members volunteered to participate in this study at Beth Israel Deaconess Medical Center, Boston, MA. Each participant completed five VR OR simulation trials; the final two trials incorporated AI assistance. The AI-guided VR OR fire simulator was validated through a human-subject study conducted at the SAGES 2019 Learning Center. Primary outcomes were performance scores, number of attempts needed to pass, and pre- and post-survey results describing participant confidence and experiences. Differences across professional or training role were assessed using chi-square tests and analyses of variance. Differences in pass rates over time were assessed using repeated measures logistic regression.

Results: 180 participants completed simulation testing; 170 (94.4\%) completed surveys. Participants included surgeons (17.2\%), anesthesiologists (10.0\%), allied health professionals $(41.7 \%)$, and medical trainees $(31.1 \%)$. Prior to training, $45.4 \%$ of participants reported feeling moderately or very confident, $29.2 \%$ only a little confident, $24.6 \%$ not at all confident in their ability to respond to an OR fire. Eight participants $(4.4 \%)$ responded safely on the first simulation attempt. Forty-three participants (23.9\%) passed by the third attempt (VR only); an additional 97 participants $(53.9 \%)$ passed within the 4th-5th attempt (VR with AI assistance).

Conclusions: Providers are unprepared to respond to OR fires. VR-based training provides a practical platform for individuals to improve their knowledge and performance in high acuity situations such as the management of OR fires. With repetitive practice within a VR OR and with AI assistance, $78 \%$ of participants in our study are able to achieve competency. VR simulation with AI guidance is a feasible, innovative, and an effective approach for individuals to assess their knowledge and improve performance of OR fire management in a stressful and realistic environment. 
S137

\section{CONSTRUCT VALIDITY OF SIMULATION-BASED OBJECTIVE STRUCTURED CLINICAL EXAMINATION SCENARIOS FOR EVALUATION OF SURGICAL SKILLS IN TRAUMA.}

Catalina Ortiz, $\mathrm{MD}^{1}$; Francisca Belmar, $\mathrm{MD}^{1}$; Ivan Paul, MD ${ }^{1}$; Javier Vela, MD ${ }^{1}$; Caterina Contreras, $\mathrm{MD}^{1}$; Cristian Jarry, $\mathrm{MD}^{1}$; Martin Inzunza, $\mathrm{MD}^{1}$; Ruben Avila, $\mathrm{MD}^{1}$; José Luis Quezada, $\mathrm{MD}^{1}$; Rodrigo Tejos, $\mathrm{MD}^{1}$; Pablo Ottolino, $\mathrm{MD}^{2}$; Julian Varas, MD ${ }^{1}$; Nicolás Jarufe, $\mathrm{MD}^{1}$; Pablo Achurra, $\mathrm{MD}^{1}$; ${ }^{1}$ Pontificia Universidad Católica de Chile; ${ }^{2}$ Hospital Dr. Sótero del Río.

Introduction: Trauma is one of the main causes of death worldwide and appropriate surgical care is crucial to impact mortality. However, resident performed trauma cases have descended in the last 10 years. Simulation has demonstrated efficiency in training and evaluation of practical skills but suitable validation for trauma surgery simulation models' is lacking.

Objectives: The aim of this study was to develop a construct-validated OSCE scenario for the training and evaluation of advanced surgical skills in trauma and emergency surgery.

Methods: We developed an OSCE with 6 stations for the evaluation and training of basic and advanced surgical skills based on simulation, all stations used real animal tissue. 1. Basic knots and sutures; 2. Bowel resection and anastomosis 3. Vascular end-to-end anastomosis 4 . Lung injury repair 5. Cardiac injury repair 6. Laparoscopic suturing of bowel injury.

Postgraduate year 2 (PY-2); recently graduated surgeons (RGS) and experts were assessed in each station. All procedures were blindly evaluated by experts using a validated general rating scale (OSATS) and time to complete the procedure. OSATS max. score was 25 for each station (150 for the complete OSCE). Mann-Whitney and Kruskal-Wallis tests were used to compare each specific nonparametric variable within each group. $\mathrm{p}<0.05$ was considered statistically significant.

Results: Eight PY-2, 8 RGS and 3 experts were assessed. Median OSATS was 78 for PY2, 113 for RGS and 150 for experts $(p<0.01)$. Average procedure time was $98 \mathrm{~min}$ for PY2, $68 \mathrm{~min}$ for RGS and $35 \mathrm{~min}$ for expert surgeons $(\mathrm{p}<0.01)$ Conclusion: The OSCE proved to adequately discriminate between levels of expertise, and therefore construct validity was established. The results show it is a valid tool for the assessment of practical skills in trauma procedures.

\section{S138}

Uncovering patient safety considerations in laparoscopic cholecystectomy using cognitive task analysis

Michael E Villarreal, MD, MBA; Clayton Rothwell, PhD; Emily Huang, MD, MA; The Ohio State University Wexner Medical Center.

Introduction: Educating residents on laparoscopic operations requires both direct teaching and deliberate practice. Attending surgeons are often systematic when performing surgery, which creates a challenge when instructing surgery residents. The aim of this study was to use cognitive task analysis to expand a laparoscopic cholecystectomy into microsteps reflecting expert surgeon cognition (perceptions, assessments, decisions, etc.) throughout the operation such that these could be better formalized and conveyed to both residents, in educational materials or assessments, and to attending surgeons, as teaching scripts.

Methods and Procedures: One surgeon, a surgical resident, and a human factors specialist conducted cognitive task analyses with three expert general surgeons and one hepatobiliary surgeon using semi-structured interviews. These interviews expanded an existing task model of laparoscopic cholecystectomy to specifically develop one centered around patient safety aspects including injury prevention, risk management, and complication detection for each step. Interview analysis resulted in an expanded task diagram.

Results: Cognitive task analysis expanded the current laparoscopic cholecystectomy task model from 19 to 97 microsteps. In addition to microsteps, an additional major step was identified, the planning step or step zero. Steps with the greatest number of microsteps included dissection with 15 microsteps and intraoperative cholangiogram with 10 microsteps.

Conclusion: Laparoscopic cholecystectomies are complex operations with several microsteps. Identification of these steps can lead to explicit strategies that can improve training of surgeons, with an end towards efficacy and patient safety. Interventions for surgical training and evaluation of competency can be developed from this analysis.

\section{S139}

\section{Blinded Intraoperative Skill Evaluations to Avoid Gender-Based} Bias

Poppy Addison, $\mathrm{MD}^{1}$; D’Artagnan Debow, $\mathrm{MD}^{1}$; Sam Dechario ${ }^{2}$; Gregg Husk $\mathrm{MD}^{1}$; Mark Jarrett, $\mathrm{MD}^{1}$; Julio Teixeira, $\mathrm{MD}^{1}$; Anthony Antonacci, $\mathrm{MD}^{1}$; Filippo Filicori, MD ${ }^{1} ;{ }^{1}$ Lenox Hill Hospital; ${ }^{2}$ Institute for Spine and Scoliosis, Lawrenceville, $\mathrm{NJ}$

Introduction: Gender bias has been identified consistently in written performance evaluations, resulting in men tending to receive more positive evaluations. Qualitative performance tools may provide a standardized way to evaluate surgical skill and minimize gender bias. Video-based assessment is often blinded to surgeonidentifying information, further minimizing potential bias. We hypothesized that there is no difference in operative time or GEARS scores in robotic hysterectomy for surgeons who are women vs men.

Methods: Patients undergoing robotic hysterectomies performed between June 2019 and March 2020 at 8 hospitals within the same hospital system were captured into a retrospective database. GEARS scores were assigned by crowd-sourced evaluators by a third party blinded to any surgeon- or patient-identifying information. Surgeon gender was determined from the preferred pronoun on the hospital website. The mean operative time and GEARS scores were determined for each surgeon, and a $\mathrm{t}$-test was used to compare the mean values for each group. Two-tailed p-value $<$ 0.05 was considered significant.

Results: Seventeen women and 13 men performed a total of 188 hysterectomies; women performed $34(18 \%)$ and men performed $153(81 \%)$. Women surgeons had a higher mean operative time $(133 \pm 58$ vs $86.3 \pm 46 \mathrm{~min}, \mathrm{p}=0.024)$. There was no significant difference between the genders in total GEARS score $(20.0 \pm 0.77$ vs $20.2 \pm 0.70, p=0.42$ ) or GEARS subcomponent scores: bimanual dexterity (3.98 \pm 0.03 vs $4.00 \pm 0.03, \mathrm{p}=0.78$ ); depth perception ( $4.04 \pm 0.04$ vs $4.05 \pm 0.02, \mathrm{p}=$ $0.87)$; efficiency $(3.79 \pm 0.02$ vs $3.82 \pm 0.02, \mathrm{p}=0.54)$; force sensitivity ( $4.01 \pm$ 0.04 vs $4.05 \pm 0.05, \mathrm{p}=0.58)$; or robotic control $(4.16 \pm 0.03$ vs $4.26 \pm 0.01, \mathrm{p}=$ $0.09)$

Conclusion: Despite a significantly higher operative time, there was no difference in GEARS score between surgeons who are women vs. men performing robotic hysterectomies. Qualitative, video-based blinded assessment of skills may minimize potential gender biases when evaluating surgical skill for competency evaluation and credentialing. 
S140

\section{Development of a formative feedback tool for transanal total mesorectal excision}

Alen Antoun, MD; Faisal Al Rashid, MD; Noura Alhassan, MD; Carlos GomezGaribello, PhD; Julio F Fiore Jr, PhD; Liane S Feldman, MD; Lawrence Lee, MD, $\mathrm{PhD}$; Carmen L Mueller, MD, MEd; McGill University.

Introduction Transanal total mesorectal excision (TaTME) is a novel procedure in the treatment of rectal cancer, which provides a technical challenge that can lead to a higher risk of complications. Current training models for TaTME suggests a period of proctored cases, but no structured feedback tool exists for formative use during a trainee's learning phase. The objective of this study was to survey an international group of TaTME experts and develop a formative feedback tool for the transanal portion of TaTME

Methods An itemized list of TaTME steps was created using review of pre-recorded TaTME videos, literature search and consultation with a board-certified colorectal surgeon with formal training in TaTME. A two-round Delphi study was conducted to determine the items to be included in the final formative feedback tool. Participants in the Delphi study were surgeon members of the Canadian TaTME Collaborative $(\mathrm{CaTaCo})$ or experts nominated by members of the $\mathrm{CaTaCo}$. Participants rated each step using a Likert scale from 1 (Not relevant) to 5 (Very relevant) with the option to suggest additional steps. Responses to the first round were summarized and integrated into the next round of the study, in which participants rated the revised list of steps. Consensus was defined as $>80 \%$ of participants rating the step as 4 or 5 out of 5. Items were combined when appropriate to avoid redundancy and rating anchors describing performance for each item on a 5-point scale were developed for all steps to complete the final feedback tool (Fig. 1). The final tool was sent to a group of colorectal surgeons along with a survey on its feasibility and usefulness.

Results Twenty-six TaTME experts were contacted for participation. Fifteen experts $(58 \%)$ participated in the first round of the study, and eleven $(42 \%)$ participated in the second round. Participants included surgeons from Canada (10,67\%), USA (4, $27 \%$ ) and Belgium $(1,7 \%)$, and the majority $(14,93 \%)$ had completed fellowship training in colorectal surgery. The first round of the Delphi study contained 34 items, and 32 items met inclusion criteria after the second round. Redundant items were combined into 14 items that comprised the final tool. Out of eight respondents to the feasibility survey, all believed the feedback tool enhances the feedback of learners and would use it for training purposes if available.

Conclusion This work describes the development of a novel formative feedback tool specific to TaTME based on expert consensus.

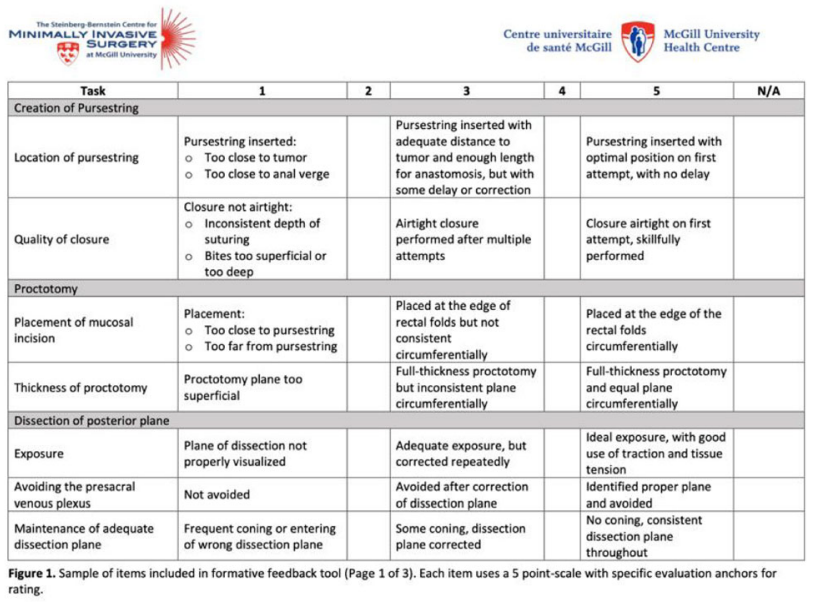

S141

\section{Validation Study of the Fundamentals of Laparoscopic Surgery} Program

Maryam Wagner, $\mathrm{PhD}^{1}$; Carlos Gomez-Garibello, $\mathrm{PhD}^{1}$; Neal Seymour, $\mathrm{MD}^{2}$; Allan Okrainec, MD ${ }^{3}$; Melina M Vassiliou, $\mathrm{MD}^{1} ;{ }^{1} \mathrm{McGill}$ University; ${ }^{2}$ University of Massachusetts; ${ }^{3}$ University of Toronto.

Introduction: The purpose of this research was to conduct a validation study of the FLS Program using an argument-based approach. The FLS program teaches and assesses the fundamental knowledge and skills required in basic laparoscopy. Since its initiation 15 years ago, over 10,000 candidates have completed the program across 30 countries. This contemporary approach to validity afforded the opportunity to generate claims about FLS by examining the past and present uses and interpretations of FLS, and synthesizing the findings to guide the future of FLS and maintain the value of the program.

Methods: The validation design needed to capture the complexities of the context, construct, and the perspectives of numerous stakeholders including surgeons, residents, program directors, SAGES staff, and exam proctors. We applied an argumentbased validity approach by: 1) Identifying validity assumptions underlying the uses of FLS and the generation of information from it; 2) Evaluating validity assumptions by directing interpretation towards the uses and effects of the program; 3) Developing research questions directed towards the overall inquiry goal; 4) Gathering data to answer research; 5) Generating validity claims based on evidence; and 6) Identifying counterclaims and presenting rebuttals. Evidence was generated through a literature review; proctor-training observations, semi-structured interviews; thinkaloud interviews; analysis of test performance data ( $>13,500$ test-takers); and retreats with experts. Various analytic approaches were employed including inductive and deductive coding, task analyses, and psychometric analyses. A mixedmethods approach was used to integrate findings across data sources.

Results: Six validity claims were generated about the contributions of FLS to education, definitions of laparoscopic surgery and its underlying competencies, tes design characteristics and uses of test information: 1) FLS is highly valuable and has potential for residency training; 2) The definition of"Fundamental" has changed since the program's inception; 3 ) The manual skills are an integral part of FLS, but some components need to be revised; 4) The metrics used in the manual skills should be revised; 5) The cognitive exam measures knowledge in laparoscopy, but the assessment may need to be more challenging, and 6) There is limited integration between the components of FLS.

Conclusions: All sound educational decisions emanating from assessments depend on trustworthy information generated from robust validation studies. This study employed a contemporary approach that generated validity evidence about the uses and interpretations of FLS. These findings will be used to update FLS and ensure that the program remains at the forefront of surgical education. 
S142

Development of the Comprehensive Operating Room Ergonomics (CORE) program: Preventing Surgeon Work-Related Musculoskeletal Disorders

Pamela C Hess ${ }^{1}$; Dimitrios I Athanasiadis, $\mathrm{MD}^{2}$; Sara Monfared, $\mathrm{MD}^{2}$; Penelope A Moyers ${ }^{3}$; Nicole K Lee, $\mathrm{MD}^{2}$; Dimitrios Stefanidis, $\mathrm{MD}, \mathrm{PhD}^{2}$; ${ }^{1}$ Department of Rehabilitation Services, Indiana University, Indianapolis, IN, USA; ${ }^{2}$ Department of Surgery, Indiana University School of Medicine, Indianapolis, IN, USA; ${ }^{3}$ School of Occupational Therapy, Indianapolis University, Indianapolis, IN, USA

Objective of the study: Work-related musculoskeletal disorders (WMSD) have been increasingly recognized to affect surgeons. Several interventions have been proposed to reduce WMSD incidence, but few follow a holistic, occupational therapy-oriented approach that addresses biomechanical, psychophysical, and psychosocial risk factors. The aim of this study was to design, implement, and assess the effectiveness of the Comprehensive Operating Room Ergonomics (CORE) program for surgeons, a novel evidence-based ergonomics intervention grounded on sound occupational therapy principles.

Methods and procedures: A six-step theory-based intervention mapping protocol guided the design and implementation of the CORE program (Fig. 1). CORE program outcomes were assessed via a mixed-methods approach. Qualitative data (semi-structured interviews and OR observations of surgeons) were coded to produce themes while for quantitative data (postural assessments using the Rapid Upper Limb Assessment-RULA) a linear regression mixed-effect model was applied.

Results: Six surgeons, three females and three males, with an average of $11 \pm$ 6.7 years of attending-level experience were included in this study. The baseline observations were 12 (two observations for each participant), while the post-CORE implementation observations were 20 (at least two for each participant). The change in RULA scores between the pre- $(5.0 \pm 1.1)$ and post-intervention time periods (4.0 $\pm 1.3)$ showed a significant estimated effect of $0.996(p=0.03)$. Thematic analysis of surgeon feedback, which was overwhelmingly positive, identified five themes: 1) enhanced postural awareness, 2) improved realization of the importance of the OR environment setup, 3) identification of postoperative discomfort as a consequence of certain incorrect intraoperative postures, 4) agreement on the need of formal surgical ergonomics training, and 5) interest in implementing ergonomics into everyday life. Conclusion: The CORE program, an evidence-based ergonomic holistic intervention, was successfully developed, and implemented with practicing surgeons and was shown to effectively decrease ergonomic risk factors that may limit a surgeon's optimal occupational performance in the operating room.

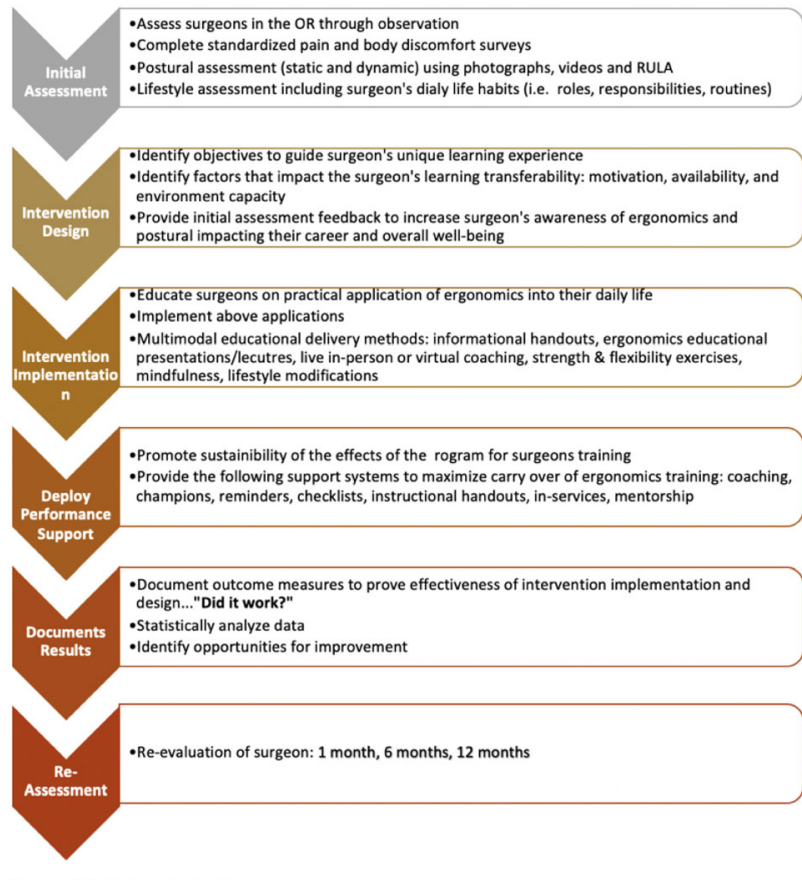

Figure 1. CORE Intervention Model
S143

\section{Hernia-To-Neck Ratio May Be Associated With Emergent Ventral Hernia Repair}

Tayler J James, MD; Paul Won, BS; Jessica Wu, MD; Lauren Hawley, MD; James D Nguyen, MD; Adrian Dobrowolsky, MD; Kamran Samakar, MD; University of Southern California.

Background: The ratio of hernia size to fascial defect size, known as the hernia-toneck ratio (HNR), has been proposed as a novel predictive factor for ventral hernia complications. A HNR $\geq 2.5$ has been suggested to warrant operation due to association with strangulation, incarceration, and necrosis. The aim of this study was to evaluate the association between HNR and emergent ventral hernia repair at our institution.

Methods: A retrospective cohort study was performed of consecutive patients who underwent emergent ventral hernia repair from 2017-2019. This group was compared to patients with ventral hernias followed in clinic who did not require emergent repair over an average of 20 months. HNR was calculated using a previously described method: maximal hernia sac size and maximal fascial defect size (termed "hernia neck size") were measured in the sagittal plane on CT scan. Data are described as mean \pm standard deviation.

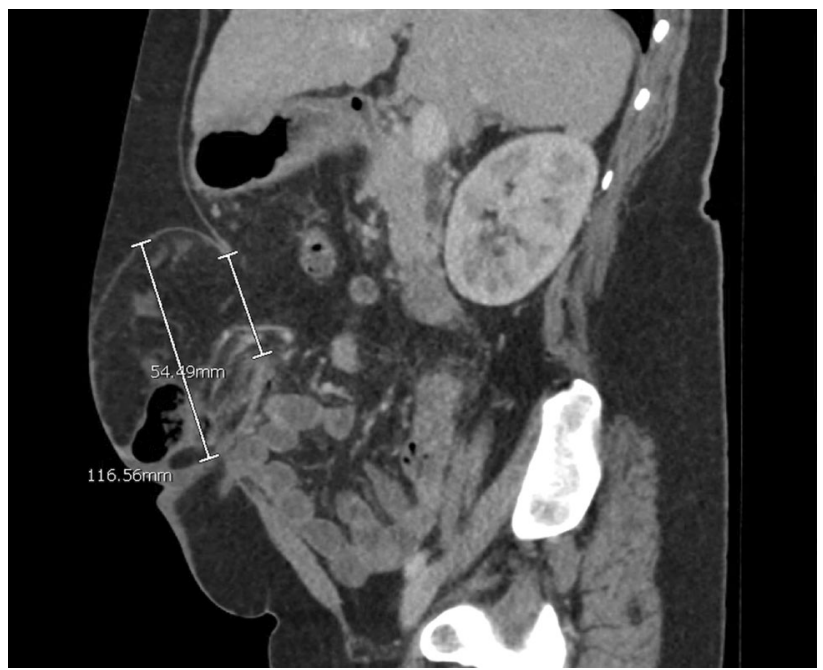

Results: 166 patients were included: 84 in the emergent repair group and 82 in the no repair group. The emergent group differed significantly from the no repair group in gender $(52.4 \%$ male vs. $34.3 \%, \mathrm{p}=0.036)$, cirrhosis $(21 \%$ vs. $8.8 \%, \mathrm{p}=0.007)$, and BMI $(34.4 \pm 9.3$ vs. $39.2 \pm 5.7, \mathrm{p}<0.001)$. The mean hernia sac and neck sizes were significantly smaller in the emergent repair group (Table). The mean hernia-toneck ratio was significantly larger in the emergent repair group at $2.6 \pm 1.1$ vs. $2.0 \pm$ $1.1, \mathrm{p}=0.001$.

CT scans were compared across time in patients in the emergent group who had a previous scan in addition to the scan at time of surgery $(n=65)$ to assess for change in HNR over time. There was no difference in mean HNR over the average time between scans of 17.3 months $(2.5 \pm 11$ vs. $2.6 \pm 1.1, \mathrm{p}=0.296)$.

No repair $(n=82) \quad$ Emergent repair $(n=84) \quad$ p value

\begin{tabular}{lccc}
\hline Mean hernia sac size $(\mathrm{mm})$ & $81.2 \pm 61.6$ & $61.9 \pm 36.3$ & $\mathbf{0 . 0 3 8}$ \\
Mean hernia neck size $(\mathrm{mm})$ & $48.9 \pm 46.6$ & $27.3 \pm 16.0$ & $\mathbf{0 . 0 0 1}$ \\
Mean hernia-neck-ratio & $2.0 \pm 1.0$ & $2.6 \pm 1.1$ & $\mathbf{0 . 0 0 1}$ \\
\hline
\end{tabular}

Conclusion: Our study suggests that HNR may be associated with emergent hernia repair, in line with prior studies proposing a HNR cutoff of $\geq 2.5$. Additionally, HNR may not significantly change over time, suggesting a higher HNR might warrant surgical intervention regardless of patient symptoms. Larger studies are warranted to evaluate the true predictive value of HNR in hernia complications and emergent repair. 
S144

\section{IMPACT OF DELAYING COLONOSCOPIES DURING THE COVID-19 PANDEMIC ON COLORECTAL CANCER DETECTION AND PREVENTION}

Jessica Holland; Natasha Caminsky; Michelle Cwintal; Carol-Ann Vasilevsky; Nancy Morin; Gabriela Ghitulescu; Julio Faria; Allison J. Pang; Marylise Boutros; Jewish General Hospital.

Introduction: The COVID-19 pandemic required strict prioritization of health care resources, which resulted in a partial/total shutdown of endoscopy in many healthcare centers worldwide. This study aims to quantify the impact of colonoscopy shutdown on colorectal cancer detection and screening.

Methods \& Procedure: After institutional ethics approval, the endoscopy database at a tertiary-care center was queried for all colonoscopies performed from MarchJune 2020, corresponding to the first wave of the pandemic, and colonoscopies performed in March-June 2019 (non-pandemic period). The indications, cancer and adenoma detection rates, as well as the prioritization of urgent procedures were compared between the two periods. Multivariable logistic regressions were used to study the association of the pandemic period with the outcomes of interest.

Results/Outcomes: In the non-pandemic period, 2,515 colonoscopes were performed while only 462 were performed during the pandemic period, representing an absolute reduction by $82 \%$. Surveillance colonoscopies in high-risk patients was reduced from 848 to 114 colonoscopies, representing an $87 \%$ absolute reduction. For screening colonoscopies in high-risk patients, only 17 patients were screened compared to 303 in 2019, a 94\% reduction. Overall, screening and surveillance were reduced from 61 to $47 \%$ ( $\mathrm{p}<0.0001$ ) of colonoscopies performed. Emergency access to colonoscopy was preserved with a median time to endoscopy of $<1$ day (IQR 0,1). An increase in proportion of colonoscopies performed in the pandemic period for urgent indications $(4.7 \mathrm{vs} 27.1 \%(\mathrm{p}<0.001))$ and as an inpatient $(0.5 \% \mathrm{vs}$. $13.6 \%$, $(\mathrm{p}<0.0001))$ was observed. In the non-pandemic period, $44(1.7 \%)$ patients were diagnosed with cancers and adenomas were removed in 766 patients $(30.5 \%)$; whereas during the pandemic period, $18(3.9 \%)$ cancers were diagnosed and 142 patients $(30.7 \%)$ had adenomas, resulting in an absolute reduction of 26 cancers and adenomas in 624 patients. On multivariate regression, the pandemic era remained independently associated with increased cancer detection (OR 2.12, 95\% CI 1.18-3.80) and an urgent colonoscopy (OR 6.58, 95\% CI 4.81-9.09), but was not associated with adenoma detection (OR $1.06,95 \%$ CI $0.84-1.35$ ).

Conclusions: Restriction of access to colonoscopy has resulted in a significan reduction in screening and surveillance even in high risk patients. While prioritization has increased the rate of cancers detected, there remains a significant reduction in cancer detection resulting in a delay in the diagnosis for these patients. Future studies will determine the ramifications of the decrease in surveillance on patients whose colonoscopies were delayed because of the pandemic

\section{S145}

Identification of 13 Novel Susceptibility Loci for Diaphragmatic Hernia Development and Their Biological and Clinical Implications: Results from the UK Biobank

Michelle Campbell, MD; Jun Wei, PhD; Mikhail Attaar, MD; Hoover Wu, MD Harry J Wong, MD; Michael Ujiki, MD; Jianfeng Xu, DrPH; NorthShore University Health System.

Introduction: Genetic contributions to hernia development are incompletely understood. To date, there is no existing published genome-wide association study (GWAS) for diaphragmatic hernia. The purpose of this study is to perform the first comprehensive GWAS for diaphragmatic hernia using a large population-based cohort in the UK Biobank (UKB).

Methods and Procedures: A two-stage GWAS (discovery and confirmation) was performed for diaphragmatic hernia from the UKB. Briefly, 275,549 and 91,850 subjects were randomly selected for association tests in Stages 1 and 2, respectively. Association tests between 8,568,156 SNPs (genotyped or imputed with MAF $>0.01$ ) in the autosomal genome and diaphragmatic hernia were performed in Stage 1. SNPs with $p<1 \times 10-5$ were selected for confirmation in Stage 2, and those with $p<0.05$ and with the same direction of association as Stage 1 were selected for combined association tests in subjects of these two stages; SNPs with combined $p<5 \times 10-8$ were considered as GWAS-significant. LD clumping analysis was performed to identify genetically independent chromosomal regions (loci). A genetic risk score (GRS) was used to measure the cumulative risk of independent SNPs in 91,849 additional subjects using odds ratios (ORs) from Stages 1 and 2 .

Results: A total of 36,351 patients were identified with the diagnosis of diaphragmatic hernia (ICD-10 K44); $43.25 \%$ of patients were male. In the Stage 1 analysis comprising $60 \%$ of the cohort, 2,654 SNPs (with $p<1 \times 10-5$ ) were identified as associated with diaphragmatic hernia. Of these, 338 SNPs (with $p<0.05$ ) were confirmed in Stage 2. In combined analysis, 245 SNPs reached GWAS significance. LD clumping analysis revealed 13 independent loci associated with diaphragmatic hernia. Among these loci, two have been previously reported associating with inguinal hernia at 2p16 (rs181661155, in the regions of EFEMP1) and at $11 \mathrm{p} 13$ (rs5030123, in the regions of WT1). eQTL analysis suggested that gene expressions of CRLF1, UBA52, and CALD1 are significantly associated with these loci and might be involved in pathogenesis of diaphragmatic hernia. GRS from these loci showed significant increase in cases compared to controls $(p<1 \mathrm{e}-16)$, and is associated with increased risk of diaphragmatic hernia $(p<1 \mathrm{e}-7)$.

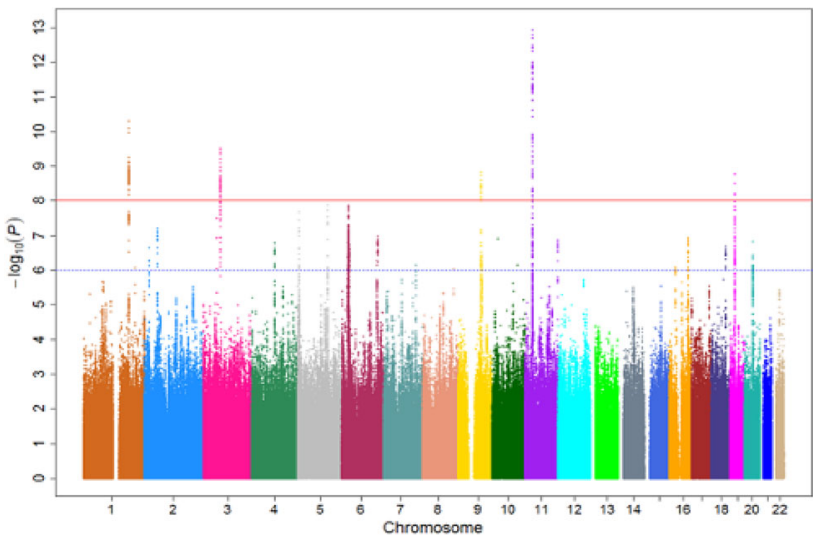

Figure 1 Manhattan plot depicting loci associated with diaphragmatic hernia Conclusions: We identified 245 SNPs at 13 novel susceptibility loci associated with diaphragmatic hernia in a large population-based cohort. The relevance of these results is two-fold. First, offering biological insight into pathogenetic mechanisms of diaphragmatic hernia development. Second, providing clinical utility in the form of genetic risk scores which may be used in risk-stratification and other clinical prediction models. 


\section{S146}

\section{Gastroesophageal junction compliance using Impedance Planimetry (EndoFLIP ${ }^{\mathrm{TM}}$ ) predicts dysphagia after fundoplication}

Hoover $\mathrm{Wu}, \mathrm{MD}^{1}$; Mikhail Attaar, MD ${ }^{1}$; Harry J Wong, MD ${ }^{1}$; Michelle Campbell, $\mathrm{MD}^{1}$; Kristine Kuchta, $\mathrm{MS}^{2}$; Woody Denham, $\mathrm{MD}^{2}$; Steven Haggerty, $\mathrm{MD}^{2}$; John Linn, $\mathrm{MD}^{2}$; Michael B Ujiki, $\mathrm{MD}^{2}$; ${ }^{1}$ Department of Surgery, University of Chicago; ${ }^{2}$ Department of Surgery, NorthShore University HealthSystem, Evanston, IL.

Introduction: Compliance is defined as the ability of a hollow organ to dilate and increase in volume with an increase in pressure, an accurate representation of food bolus transit through the gastroesophageal junction (GEJ). This can be calculated as change in volume over change in pressure, whereas distensibility is calculated by dividing cross sectional area over pressure. The functional luminal imaging probe (FLIP) can be used to describe GEJ compliance during anti-reflux surgery. We hypothesize that GEJ compliance is a better predictor of long term outcomes than distensibility index (DI).

Methods and Procedures: A retrospective review of a prospectively maintained quality database was performed. Patients with FLIP measurements during laparoscopic fundoplication between August 2018 and March 2020 were included. GEJ Compliance and DI were measured without pneumoperitoneum with a $40 \mathrm{~mL}$ balloon fill after hernia reduction, cruroplasty, and fundoplication. Dysphagia was measured using patient surveys up to two years after surgery. A scatter plot was used to identify correlation between compliance and DI. Comparisons of measurements between time points were made using paired t-tests. Spearman's correlation coefficients $(\rho)$, Wilcoxon rank-sum and chi-square tests were used to evaluate associations between FLIP measurements and outcomes.

Results: Eighty patients underwent laparoscopic fundoplication (29\% Nissen, $71 \%$ Toupet) and had FLIP measurements. Compliance is strongly associated with DI $(\mathrm{r}=$ 0.94) and comparison of measurements showed similar trends at specific time points during the operation (Table 1). Compliance at the GEJ after hernia reduction was $170 \pm 71 \mathrm{~mm}^{3} / \mathrm{mmHg}$, cruroplasty $80 \pm 26 \mathrm{~mm}^{3} / \mathrm{mmHg}$, and fundoplication $89 \pm$ $30 \mathrm{~mm}^{3} / \mathrm{mmHg}$ (all comparisons $\mathrm{p}<0.05$ ). Compliance at fundoplication was inversely associated with dysphagia at two years $(\rho=-0.39, p=0.039)$. DI was also inversely associated with dysphagia $(\rho=-0.39, p=0.039)$. Patients with a compliance of $\leq 54 \mathrm{~mm}^{3} / \mathrm{mmHg}$ at fundoplication reported more severe dysphagia compared to those with compliance of 54.1-86.0 and $>86(1.5 \pm 0.5$ vs. $1.1 \pm 0.3$ vs. $1.0 \pm 0.0, p=0.017)$, as well as any symptoms of dysphagia ( $p=0.011$, Fig. 1$)$ Conclusions: GEJ compliance is not a better predictor of long term outcomes compared to DI. Compliance and DI are strongly associated with each other and equally predict post-operative dysphagia at two years. A compliance of greater than $54 \mathrm{~mm}^{3} / \mathrm{mmHg}$ at fundoplication is potentially ideal to avoid bothersome dysphagia. GEJ compliance is an underutilized FLIP measurement warranting further investigation during anti-reflux surgery.

Table 1.

\begin{tabular}{|ccc|}
\hline FLIP Measurements & & \\
\hline & 40ml without pneumoperitoneum \\
\hline & Compliance & DI \\
\hline & Mean \pm SD & Mean \pm SD \\
\hline Hernia Reduction (N=63) & $170 \pm 71$ & $6.7 \pm 3.3$ \\
Crural Closure (N=69) & $80 \pm 26$ & $2.9 \pm 1.3$ \\
p-value vs. Hernia Reduction & $<.0001$ & $<.0001$ \\
Fundoplication (N=73) & $89 \pm 30$ & $3.6 \pm 1.3$ \\
p-value vs. Hernia Reduction & $<.0001$ & $<.0001$ \\
p-value vs. Crural Closure & 0.0456 & 0.0002 \\
Change from HR to Fundo (N=62) & $-85 \pm 73$ & $-3.2 \pm 3.2$ \\
\hline
\end{tabular}

Compliance $\left(\mathrm{mm}^{3} / \mathrm{mmHg}\right)$

$\mathrm{DI}\left(\mathrm{mm}^{2} / \mathrm{mmHg}\right)$
Figure 1

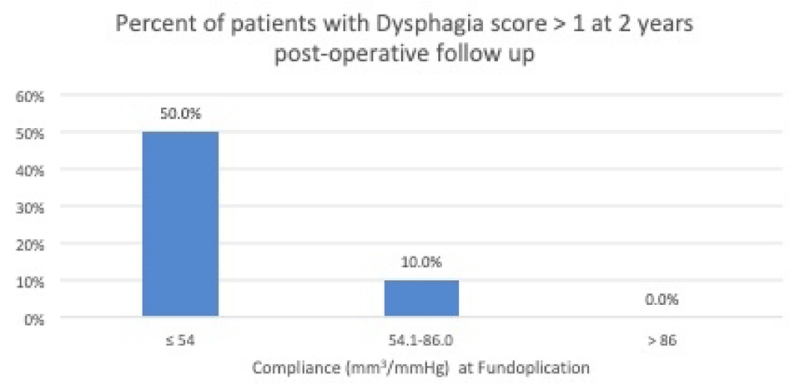

\section{S147}

\section{Anatomic location of hiatal hernia recurrence: a video-based assessment}

Maria E Linnaus, MD; Shanley Deal, MD; Jon C Gould, MD, MBA; Medical College of Wisconsin.

Intro: Hiatal hernia recurrence following surgical repair is common. Surgeons employ various techniques attempting to decrease recurrence. There is growing discussion about whether an anteriorly-oriented U-shaped mesh reinforces the part of the hiatus at highest risk for failure. We sought to define the most common anatomic location for hiatal failure to inform technical strategies to decrease recurrence rates. Methods: Retrospective chart review and video analysis were performed for all recurrent hiatal hernia operations performed by a single surgeon (JG) between January 2013 and April 2020. All procedures were video recorded and retrospectively analyzed for hiatal recurrence location. Hiatal recurrences were defined by anatomic quadrants as left-anterior, right-anterior, right-posterior, and right-anterior Recurrences on both left and right on either the anterior or posterior portion of the hiatus were simply classified as 'anterior' or 'posterior' respectively. Three or more quadrants were defined as circumferential. Descriptive statistics and chi-square analysis were utilized.

Results: One-hundred-and-thirty patients met inclusion criteria. Median age was 58 years (IQR49-66) and 78\% $(\mathrm{n}=102)$ were female. Median time to recurrence from previous operation was 60 months (IQR19.5-132). First-time recurrent repairs were most common (97 first-time (74\%); $23(18 \%)$ second-time; and $10(8 \%)$ three or more recurrences). Non-mutually exclusive indications for reoperation were GERD $(\mathrm{n}=100,76 \%)$, dysphagia $(\mathrm{n}=57,44 \%)$, and epigastric/chest pain $(\mathrm{n}=8$, $6 \%$ ). Twenty-one $(16 \%)$ patients had previously placed mesh at the hiatus. Al reoperative cases were completed laparoscopically. Patients underwent recurrent hiatal hernia repair and reoperative Toupet $(57 \%)$; hiatal hernia repair and reoperative Nissen (18\%), hiatal hernia repair, takedown of fundoplication and conversion to Roux en Y gastric bypass (20\%), and gastropexy alone (4\%). Median operative time was $198 \mathrm{~min}$ (IQR153-237). Forty-three (33\%) patients had a bioabsorbable mesh placed at the hiatus posteriorly (U-shaped, opening anteriorly) during their reoperative procedure for crural reinforcement. Video analysis revealed anterior recurrences were most common $(n=87,66 \%)$, followed by circumferential $(n=38$, $29 \%)$. Two patients had a single-quadrant left-anterior recurrence $(2 \%)$, while two had a purely posterior recurrence $(2 \%)$. One had a right-sided recurrence (right anterior and posterior). The prior number of hiatal surgeries did not predict anatomic recurrence location $(\mathrm{p}=0.91)$. Mesh placement at prior hiatal repair did not influence the location of recurrence $(\mathrm{p}=0.25)$.

Conclusion: When hiatal hernias recur, the anterior portion of the hiatus is most likely to fail. Outcomes following techniques designed to reinforce the anterior hiatus should be explored. 


\section{S148}

\section{LONG-TERM PATIENT-REPORTED OUTCOMES OF LAPAROSCOPIC MAGNETIC SPHINCTER AUGMENTATION VERSUS NISSEN FUNDOPLICATION: A 5-YEAR FOLLOW-UP STUDY}

Sean M O'Neill, MD, PhD; Anahita D Jalilvand; Jennifer S Colvin, MD; Kelly R Haisley, MD; Kyle A Perry, MD; The Ohio State University Wexner Medical Center.

INTRODUCTION: Laparoscopic Magnetic Sphincter Augmentation (MSA) has emerged as an alternative to Laparoscopic Nissen Fundoplication (LNF) for the management of gastroesophageal reflux disease (GERD). While short-term outcomes of MSA compare favorably to those of LNF, direct comparisons of long-term outcomes are lacking. We hypothesized that the long-term patient-reported outcomes of MSA would be similar to those achieved with LNF.

METHODS: We tested this hypothesis in a retrospective cohort who underwent primary LNF or MSA at a single institution between March 2013 and July 2015. Patients with hiatal hernias larger than $5 \mathrm{~cm}$ or significant esophageal dysmotility were excluded. Baseline demographics, preoperative testing, GERD-HRQL scores, and follow-up data were compiled in an institutional review board-approved database. The primary outcome was GERD-Health Related Quality of Life (GERDHRQL) score. Secondary outcomes included dysphagia and bloating scores, protonpump inhibitor (PPI) cessation, reoperations, and overall satisfaction with surgery. Data are expressed as mean (SD), median (IQR), or number (\%) as appropriate. For bivariate comparisons, a p-value of $<0.05$ was considered statistically significant. RESULTS: 70 patients (45 LNF and 25 MSA) met criteria for study inclusion. LNF patients had higher baseline BMI (30.4 [26.4-32.8] versus 27.1 [22.7-29.9], $\mathrm{p}=$ $0.02)$, GERD-HRQL (34 [25-40] versus 26 [19-32], $\mathrm{p}=0.02)$, and dysphagia (3 $[1-4]$ versus $2[0-3], p=0.02)$ scores. Presence of hiatal hernia, esophagitis, or Barrett's esophagus and esophageal acid exposure did not differ between groups. Long-term follow up rate (LNF: $\mathrm{n}=36(80 \%)$; MSA: $\mathrm{n}=20(80 \%), \mathrm{p}=1.0)$ and median (IQR) follow-up interval in months (LNF: 65 (62-69); MSA: 68 (65-74), p = 0.08 ) were similar in both groups. GERD-HRQL scores improved from 34 [25-40] at baseline to $7.5[2.5-14]$ at follow-up following LNF $(\mathrm{p}<0.01)$ and from 26 [19-33] to 9.0 [4-14] after MSA ( $p<0.001)$. Long-term GERD-HRQL scores $(\mathrm{p}=$ $0.68)$, PPI usage (33\% versus $40 \%, \mathrm{p}=0.62)$ and need for redo surgery $(8 \%(\mathrm{n}=3)$ versus $24 \%(\mathrm{n}=5), \mathrm{p}=0.10)$ did not differ significantly between groups. Dysphagia scores were similar $(0[0-2]$ versus $1[0-2], \mathrm{p}=0.96)$, and bloating was more common after LNF (3.0 [1-4] versus $1.5[0.5-3.0], \mathrm{p}=0.08)$, although this did not reach statistical significance. Eighty-nine percent of LNF patients reported satisfaction with the procedure, compared to $70 \%$ of MSA patients $(\mathrm{p}=0.09)$.

CONCLUSIONS: Magnetic sphincter augmentation appears to offer similar longterm improvement in disease-specific quality of life as LNF. For MSA, there was a trend toward reduced long-term bloating compared to LNF, but need for reoperation and device removal may be associated with patient dissatisfaction.

\section{S150}

\section{Finding Relief for the Self-Conscious Esophagus: LARS} and EHAS

Charles Hill, MD; Tom Crijns, MD; Yousef Nofal; Stephanie Doggett, PA; Katherine Walsh; Derek Yan; Jeremiah Alexander; Cole Holan; Elisa Furay, MD; F. P Buckley, MD; Dell Seton Medical Center at Univ of Texas at Austin.

Introduction: Measures of mood and effective coping strategies have notable correlations with quality of life and treatment responses. There is evidence that patients with previously diagnosed anxiety disorders have less improvement in patient-reported outcomes (PROs) after laparoscopic anti-reflux surgery (LARS) and that objective pathology does not correlate well with symptom severity. We were interested in investigating whether anxiety and hypervigilance, as measured preoperatively with the Esophageal Hypervigilance Anxiety Scale (EHAS), is associated with the improvement in GERD-specific PROs and EHAS scores 6 months after LARS.

Methods: We performed a retrospective cohort study of 102 adult patients (31\% men, average age 64) who underwent LARS. In the preoperative evaluation, baseline Gastroesophageal Reflux Disease-Health Related Quality of Life (GERD-HRQL), Laryngopharyngeal Reflux Symptom Index (LPR-RSI) and EHAS scores were collected in addition to the standard reflux workup, including endoscopy, manometry, barium swallow, and $\mathrm{pH}$ study. At 6 months postoperatively, patients completed repeat GERD-HRQL, LPR-RSI, and EHAS surveys. We then analyzed for surgical and patient-related factors associated with improvement in the 6-month postoperative GERD-HRQL and LPR-RSI scores.

Results: There was a statistically significant decrease in the GERD-HRQL ( 25 vs 2 , $\mathrm{p}<0.001)$, LPR-RSI (17 vs 3, p < 0.001) and EHAS (34 vs $15, \mathrm{p}<0.001) 6$ months after LARS. On multivariable linear regression, a higher baseline EHAS score was independently associated with a greater improvement in GERD-HRQL $(\beta 0.35, \mathrm{p}<$ $0.001)$ and LPR-RSI $(\beta 0.19, \mathrm{p}=0.03) 6$-months after LARS. Additionally, the degree of improvement in EHAS, GERD-HRQL, and LPR-RSI was not influenced by the type of LARS performed or by the severity of disease.

Conclusion: These findings are consistent with literature suggesting that measures of psychoemotional health correlate better with symptom intensity than objective pathology. Our previous findings with this cohort showed that patients with a higher EHAS score have greater symptom severity and lower quality of life at baseline. Novel findings to this study are that patients with a higher preoperative EHAS, a measure of psychoemotional health, actually benefitted more from surgery and not less, which has been the traditional view in the literature. Future studies are warranted to establish directionality and explore the role of preoperative cognitive behavioral therapy with LARS for patients with significant symptoms of hypervigilance and anxiety. 
S151

\section{Impedance Planimetry (EndoFLIP ${ }^{\mathrm{TM}}$ ) after Magnetic Sphincter Augmentation (LINX@)}

Hoover Wu, MD ${ }^{1}$; Mikhail Attaar, MD ${ }^{1}$; Harry J Wong, MD ${ }^{1}$; Michelle Campbell, $\mathrm{MD}^{1}$; Kristine Kuchta, $\mathrm{MS}^{2}$; Woody Denham, $\mathrm{MD}^{2}$; Steven Haggerty, $\mathrm{MD}^{2} ;$ John Linn, $\mathrm{MD}^{2}$; Michael B Ujiki, $\mathrm{MD}^{2} ;{ }^{1}$ Department of Surgery, University of Chicago, $\mathrm{IL} ;{ }^{2}$ Department of Surgery, NorthShore University HealthSystem, Evanston, IL.

Introduction: The use of functional luminal imaging probe (FLIP) during laparoscopic fundoplication (LF) for gastroesophageal reflux disease is well described. However, there is a lack of data on FLIP measurements during magnetic sphincter augmentation (MSA). The aims of the present study are to report our institutional experience in performing FLIP during MSA and compare these measurements to those obtained during LF.

Methods and Procedures: A retrospective review of a prospectively maintained quality database was performed. Patients who underwent MSA or LF and had FLIP measurements between April 2018 and June 2020 were included. FLIP measurements at the gastroesophageal junction (GEJ) were recorded without pneumoperitoneum at $40 \mathrm{~mL}$ balloon fill after hernia reduction, cruroplasty, and MSA or fundoplication. Reflux Symptom Index (RSI), GERD-HRQL, and Dysphagia Score were collected up to two years. Group comparisons were made using two-tailed Wilcoxon rank-sum and chi-square tests, with statistical significance of $\mathrm{p}<0.05$.

Results: Nineteen patients underwent MSA and 76 patients underwent LF (68\% Toupet, $32 \%$ Nissen). At hernia reduction and cruroplasty, MSA and LF measurements were similar $(\mathrm{p}>0.05)$. Minimum diameter (Dmin), balloon pressure, cross sectional area (CSA), and distensibility index (DI) were lower after MSA device placement compared to LF $(\mathrm{p}<0.05)$ (Table 1). Post-operative follow-up for each group had no differences in RSI, GERD-HRQL, and Dysphagia Score ( $\mathrm{p}>0.05)$. Conclusions: Intraoperative impedance planimetry provided objective information regarding the geometry of the GEJ during MSA. The ring of magnetic beads restores the anti-reflux barrier and transiently opens with food bolus and belching. The magnetic force of the beads may explain why the DI after MSA is lower yet quality of life and Dysphagia Score are no different than LF.

Table 1.

\begin{tabular}{|c|c|c|c|}
\hline & LINX & LF & p-value \\
\hline \multicolumn{4}{|l|}{ Hernia Reduction } \\
\hline Dmin, $\mathrm{mm}$ & $12.9 \pm 2.8$ & $13.7 \pm 2.7$ & 0.367 \\
\hline Pressure, $\mathrm{mmHg}$ & $26 \pm 7$ & $26 \pm 8$ & 0.755 \\
\hline $\mathrm{CSA}_{\mathrm{p}} \mathrm{mm}^{2}$ & $136 \pm 56$ & $151 \pm 64$ & 0.441 \\
\hline $\mathrm{Dl}_{r} \mathrm{~mm}^{2} / \mathrm{mmHg}$ & $5.5 \pm 2.9$ & $6.3 \pm 2.9$ & 0.284 \\
\hline \multicolumn{4}{|l|}{ Crural Closure } \\
\hline Dmin, mm & $10.1 \pm 1.8$ & $10.3 \pm 2.0$ & 0.860 \\
\hline Pressure, $\mathrm{mmHg}$ & $34 \pm 7$ & $32 \pm 7$ & 0.249 \\
\hline $\mathrm{CSA}_{p} \mathrm{~mm}^{2}$ & $83 \pm 27$ & $87 \pm 34$ & 0.860 \\
\hline $\mathrm{Dl}_{r} \mathrm{~mm}^{2} / \mathrm{mmHg}$ & $2.6 \pm 1.0$ & $2.9 \pm 1.3$ & 0.598 \\
\hline \multicolumn{4}{|l|}{ LINX/LF } \\
\hline Dmin, mm & $9.6 \pm 1.6$ & $12.0 \pm 2.5$ & $<0.001$ \\
\hline Pressure, $\mathrm{mmHg}$ & $39 \pm 7$ & $34 \pm 9$ & 0.030 \\
\hline $\mathrm{CSA}_{\mathrm{i}} \mathrm{mm}^{2}$ & $74 \pm 24$ & $119 \pm 46$ & $<0.001$ \\
\hline $\mathrm{Dl}_{r} \mathrm{~mm}^{2} / \mathrm{mmHg}$ & $2.0 \pm 0.7$ & $3.5 \pm 1.3$ & $<0.001$ \\
\hline
\end{tabular}

\section{S152}

3D-thoracoscope improves mediastinal lymph node dissection and prognosis in the patients underwent prone position thoracoscopic esophagectomy

Kazuo Koyanagi, MD, PhD; Soji Ozawa, MD, PhD; Yamato Ninomiya, MD, PhD; Kentaro Yatabe, MD; Miho Yamamoto, MD, PhD; Tadashi Higuchi, MD; Kohei Tajima, MD; Kohei Kanamori, MD; Department of Gastroenterological Surgery, Tokai University School of Medicine.

Introduction: Aim of the study was to assess the superiority of lymph node dissection (LND) and surgical outcomes after prone-position thoracoscopic esophagectomy (TE) using 3D flexible thoracoscope.

Methods: The 338 patients who underwent TE for esophageal cancer between 2009 and 2017 were enrolled. All patients underwent 3-field LND. 2D flexible thoracoscope was used in 184 cases (2D) and 3D flexible thoracoscope in 154 cases (3D). Surgical outcomes, number of lymph node (LN) retrieved, and prognosis were compared between the 3D group and the $2 \mathrm{D}$ group.

Results: The patients consisted of 288 males and 50 females, with a median age of 68 years. Tumor location, histology, and stage were not different between the 3D group and the $2 \mathrm{D}$ group. Thoracoscopic bleeding was significantly lower in the $3 \mathrm{D}$ group $(\mathrm{p}<0.001)$. Thoracoscopic operative time was shorter in the 3D group $(\mathrm{p}=$ 0.02 ). Total number of retrieved mediastinal LNs was significantly higher in the $3 \mathrm{D}$ group $(\mathrm{p}<0.001)$. The number of retrieved LNs in the upper thoracic zone was significantly higher in the 3D group $(\mathrm{p}<0.001)$. The number of retrieved No. 109L and No. 112 stations was significantly higher in the 3D group $(\mathrm{p}=0.001$ and $\mathrm{p}<$ $0.001)$. On the other hand, the number of retrieved No. 101L/R stations was significantly lower in the 3D group $(\mathrm{p}=0.004)$. There was no difference in the incidence of recurrent laryngeal nerve palsy, chylothorax, or postoperative hospital stay. In 331 patients excluding 7 who underwent R2 resection of primary tumor, LN recurrence occurred in $51(28 \%)$ and $31(21 \%)$ patients in the 3D group and the 2D group. Recurrence at No. 109L station was significantly lower in the 3D group $(\mathrm{n}=$ $4,2.7 \%)$ than that in the $2 \mathrm{D}$ group $(\mathrm{n}=14,7.7 \%)(\mathrm{p}=0.02)$. In the 290 patients excluding 41 who died of other diseases, OS was significantly better in the 3D group than that in the $2 \mathrm{D}$ group $(\mathrm{p}=0.01)$.

Conclusions: Prone-position TE using 3D thoracoscope can improve the accuracy of mediastinal LND and postoperative survival without increasing the postoperative complications in esophageal cancer patients. 


\section{$\mathbf{S 1 5 3}$}

\section{Assessment of Muscle Activity and Fatigue during Laparoscopic Surgery}

Tegan J Thurston ${ }^{1}$; James P Dolan, $\mathrm{MD}^{2}$; Farah Husein, $\mathrm{MD}^{3}$; Kenneth Funk, $\mathrm{PhD}^{4}$; Charlie Borzy, $\mathrm{BS}^{2}$; Xinhui Zhu, $\mathrm{PhD}^{4} ;{ }^{1}$ School of Chemical, Biological, and Environmental Engineering, Oregon State University, Corvallis, OR, USA; ${ }^{2}$ Division of Gastrointestinal and General Surgery, Department of Surgery, Oregon Health and Science University, Portland, OR, USA ${ }^{3}$ Division of Bariatric Surgery, Department of Surgery, Oregon Health and Science University, Portland, OR, USA; ${ }^{4}$ School of Mechanical, Industrial, and Manufacturing Engineering, Oregon State University, Corvallis, OR, USA.

Background: Cumulative musculoskeletal stress during operative procedures can contribute to the development of chronic musculoskeletal injury among surgeons. This is a particular concern in laparoscopic specialties, where trainees may be at a detriment by learning poor operative posture or technique early in training. The aim of this study was to conduct an initial investigation of the physical stress encountered during the conduct of foregut laparoscopic surgery. These results might better inform surgical training programs in regard to specific areas of physical stress during foregut laparoscopic procedures.

Methods: Subjects were divided into two groups based on their experience level. The high experience (HE) group consisted of two academic laparoscopic foregut surgeons with over 30 combined years of advanced laparoscopic practice. The low experience group (LE) consisted of two minimally-invasive fellows (one year advanced laparoscopic practice) and a surgical chief resident (no advanced laparoscopic practice). Nine distinct foregut laparoscopic procedures were observed for data collection within these groups. Individual electromyographic activity was collected at ten different anatomic locations, including the bilateral neck, shoulders, biceps, triceps, and lower back for each procedure. Physical workload was measured using percent reference volunteer contractions (\% RVC) for each surgeon's muscle activities. Fatigue development was assessed by the changes of median frequencies of electromyography (EMG) data between two consecutive cases. At the end of surgery, all subjects completed a North American Space Administration-task load index (NASA-TLX) survey.

Results: All surgeons were right-hand dominant. LE surgeons experienced higher levels of \% RVC in the lower back muscles compared to the HE surgeons ( $96 \%$ vs. $38 \%$ on the right and $208 \%$ vs. $49 \%$ on the left). LE fatigue level was also higher than the HE surgeons across most muscle groups. Fatigue development after performing two consecutive cases indicated a trend in median frequency decrease for six of the ten muscle groups, the largest decrements being in the biceps and triceps. Conclusions: Surgeons were found to have high risk of musculoskeletal disorders/ injuries and develop fatigue in consecutive cases while performing MIS. Also, the higher the experience of surgeons, the lower the physical workload. Meanwhile, surgeons with different experience level would use their muscles differently. The new knowledge reported in this study can be used to design ergonomic interventions to reduce surgeons' workload. It can also alert the surgical training program of the importance of ergonomic body movements and potentially include it in their training curriculum.

\section{S154}

\section{EVALUATION OF ADDITIONAL SURGERY AFTER NONCURATIVE ENDOSCOPIC SUBMUCOSAL DISSECTION FOR EARLY GASTRIC CANCER}

Shinichiro Makimoto; Hiroyuki Yoshitake; Yutaka Mushiake; Hirofumi Shintani; Tomoya Takami; Tomoyuki Yamaguchi; Naoki Kataoka; Shouji Oura; Kishiwada Tokushukai Hospital.Background: The additional surgery after noncurative endoscopic submucosal dissection (ESD) for early gastric cancer is controversial. The present study aims to evaluate the validity of this particular surgery. Whereas the additional surgery is generally recommended for noncurative resection, there are certain patients, such as the older adults, who may be unsuitable for this procedure. We intend to clarify the factors related to the residual cancer, and, in light of this, determine whether the additional surgery would be helpful.

Methods: A total of 1483 patients underwent ESD for early gastric cancer between January 2012 and March 2020 at Kishiwada Tokushukai Hospital. Of these, 103 who underwent additional gastrectomy after noncurative ESD were retrospectively analyzed.

Results: In total, 103 patients (79 male, 24 female; median age 70 years, interquartile range [IQR] 64-75 years)were included in our study. Median tumor size was $24 \mathrm{~mm}$ (IQR $17.5-37.5 \mathrm{~mm}$ ). Factors pertaining to subjects' noncurative resection included the following: positive lymphatic invasion, $44(42.7 \%)$ patients; positive vascular invasion, $22(21.4 \%)$; positive horizontal margin, $6(5.8 \%)$; positive vertical margin, 20 (19.4\%); and submucosal invasion over $0.5 \mathrm{~mm}$ (SM2), 81 (78.6\%); and muscularis propria invasion(MP), 2(1.9\%). Distal gastrectomy (open/ laparoscopic) was performed in $60(3 / 57)$ patients, total gastrectomy (open/laparoscopic) in $29(4 / 25)$, and proximal gastrectomy (open/laparoscopic) in $11(4 / 7)$. The surgical specimen showed lymph node metastasis (LNM) in $14(13.6 \%)$ patients, and local residual cancer $(\mathrm{RC})$ in $6(5.8 \%)$. However, neither of these malignancies were observed in $85(82.5 \%)$ patients. Multivariate analysis revealed that positive lymphatic invasion $(\mathrm{P}=0.012)$ and positive ulceration $(\mathrm{P}=0.036)$ were independent risk factors for LNM, whereas positive horizontal margin $(P=0.020)$ and positive ulceration $(\mathrm{p}=0.041)$ were independent risk factors for local RC. The median follow-up period was 46 months (IQR 23-62 months). During follow-up, 3(2.9\%)patients had recurrences in the peritoneum, bone, and lymph nodes, respectively, 2 $(1.9 \%)$ patients died of gastric cancer, and $6(5.8 \%)$ died of other diseases.

Conclusion: Based on our results, we recommend additional gastrectomy with lymph node dissection for patients after noncurative ESD for early gastric cancer. Additional surgery under these circumstances had proven effective and had longterm outcomes. However, we find that it may be acceptable to follow up carefully without additional surgery for certain patients with severe comorbidity as well as older adults. 


\section{$\mathbf{S 1 5 5}$}

\section{Social Support Provided to Bariatric Surgery Patients Through a Facebook Group May Improve Weight Loss Outcomes}

Dimitrios I Athanasiadis, $\mathrm{MD}^{1}$; Rosalie A Carr, $\mathrm{MD}^{1}$; Craig Smith, $\mathrm{MD}^{2}$; Rebecca C Dirks, $\mathrm{MD}^{1}$; William Hilgendorf, $\mathrm{PhD}^{3}$; Don Selzer, $\mathrm{MD}^{1}$; Dimitrios Stefanidis, $\mathrm{MD}, \mathrm{PhD}^{1} ;{ }^{1}$ Department of Surgery, Indiana University School of Medicine, Indianapolis, IN, USA; ${ }^{2}$ Bon Secours Mercy Health, VA, USA; ${ }^{3}$ Indiana University Health North Hospital, Indianapolis, IN, USA.

Objective: Patient social support has been shown to impact outcomes after bariatric surgery. We have previously shown that a Facebook group administered by bariatric providers offers an effective alternative social support mechanism to in-person support groups with higher patient participation. Our aim was to determine whether participation in this Facebook group could improve patient outcomes after bariatric surgery.

Methods and procedures: After IRB approval, our center's Facebook group members were electronically surveyed about their perceived value of group participation and their usage frequency. We also collected patient age, sex, insurance, preoperative weight, type of procedure, hospital stay, postoperative complications, and weight loss from the electronic medical record. To assess the impact of Facebook group participation we compared patient outcomes between "frequent users" (checking the Facebook group activity at least once a week), "infrequent users", and a control group of all patients operated on during the year prior to the establishment of the Facebook group. The groups were compared using ANOVA and chi-square analyses. A multivariable analysis was undertaken to control for confounders such as age, sex, and type of procedure.

Results 250 out of 1400 Facebook group patients responded to the survey (18\%). 195 patients were frequent and 55 were infrequent users. Outcomes were compared with 211 control patients. Facebook users reported that group participation helped them do well postoperatively (3.9/5 Likert) and they felt understood by their peers (4.1/5 Likert). The groups did not differ in their baseline characteristics apart from their sex. Frequent users had a higher weight loss compared to the other groups up to 2 years postop (Table 1). On multivariable analysis, frequency of Facebook use was the main factor associated with $0.5,1$, and 2 -year weight loss.

Conclusions: Frequent participation in a Facebook support group after bariatric surgery was associated with improved early weight loss outcomes. If additional longer-term studies confirm our findings, offering similar social support groups may become essential after bariatric surgery, especially during times of social isolation when in-person social support meetings may not be feasible.

\begin{tabular}{|c|c|c|c|c|c|}
\hline & & $\begin{array}{l}\text { FREQUENT USERS } \\
(\mathrm{N}=195)\end{array}$ & $\begin{array}{l}\text { INFREQUENT USERS } \\
(\mathrm{N}=55)\end{array}$ & $\begin{array}{l}\text { CONTROL } \\
(\mathrm{N}=\mathbf{2 1 1})\end{array}$ & P-VALUE \\
\hline \multirow{2}{*}{\multicolumn{2}{|c|}{$\begin{array}{l}\text { AGE (MEAN } \pm \text { SD) } \\
\text { FEMALES (\%) }\end{array}$}} & $45.1 \pm 11.2$ & $43.8 \pm 10.2$ & $45.6 \pm 11.2$ & 0.582 \\
\hline & & $92.7 \%$ & $87.3 \%$ & $81.2 \%$ & $<0.001$ \\
\hline \multicolumn{2}{|c|}{ PRIVATE INSURANCE } & $67.0 \%$ & $67.4 \%$ & $63 \%$ & 0.667 \\
\hline & LRYGB & $128(65.6 \%)$ & $35(63.6 \%)$ & $137(64.9 \%)$ & \\
\hline PROCEDURE & LSG & $58(29.7 \%)$ & $13(23.6 \%)$ & $57(27 \%)$ & 0.281 \\
\hline (\%) & other & $9(4.6 \%)$ & $7(12.7 \%)$ & $17(8.1 \%)$ & \\
\hline \multicolumn{2}{|c|}{$\begin{array}{c}\text { HOSPITAL STAY (MEAN } \\
\pm \text { SD) }\end{array}$} & $2.0 \pm 1.4$ & $1.9 \pm 1.6$ & $2.3 \pm 2.0$ & 0.051 \\
\hline \multicolumn{2}{|c|}{ PREOPERATIVE BMI } & $47.0 \pm 8.1$ & $45.6 \pm 6.1$ & $45.9 \pm 7.6$ & 0.262 \\
\hline \multirow{2}{*}{\multicolumn{2}{|c|}{$\begin{array}{l}\text { 6-MONTH } \triangle B M I \\
1^{\text {ST }} \text { YEAR } \triangle B M I\end{array}$}} & $11.7 \pm 2.9(\mathrm{~N}=166)$ & $10.6 \pm 3.2(\mathrm{~N}=39)$ & $10.7 \pm 3.2(\mathrm{~N}=166)$ & 0.046 \\
\hline & & $14.8 \pm 4.7(\mathrm{~N}=122)$ & $13.1 \pm 4.4(\mathrm{~N}=39)$ & $13.2 \pm 4.7(\mathrm{~N}=149)$ & 0.017 \\
\hline \multicolumn{2}{|c|}{$2^{\text {NO }}$ YEAR $\triangle B M I$} & $14.3 \pm 5.0(\mathrm{~N}=47)$ & $12.2 \pm 6.0(\mathrm{~N}=26)$ & $12.7 \pm 5.7(\mathrm{~N}=128)$ & 0.048 \\
\hline \multicolumn{2}{|c|}{$3^{\text {RD }}$ YEAR $\triangle B M I$} & $12.3 \pm 4.9(\mathrm{~N}=21)$ & $10.0 \pm 7.0(\mathrm{~N}=15)$ & $11.0 \pm 5.8(\mathrm{~N}=108)$ & 0.226 \\
\hline \multicolumn{6}{|c|}{$\begin{array}{l}\text { BMI, body-mass-index; LRYGB, laparoscopic Roux-en-Y gastric bypass; LSG, laparoscopic sleeve } \\
\text { gastrectomy; SD, standard deviation }\end{array}$} \\
\hline
\end{tabular}

\section{S156}

\section{Greater Sustained Improvement in GERD-HRQL after Gastric} Bypass than Sleeve Gastrectomy

Jessica Blumhagen, MD; Paige Martinez, MS; Ellen Morrow, MD, MS; Eric Volckmann, MD; Anna Ibele, MD; University of Utah.

Background: Gastroesophageal Reflux Disease (GERD) is present in $50-70 \%$ of patients with obesity. Roux-en-Y Gastric Bypass (RYGB) has demonstrated effectiveness as treatment for GERD in patients with obesity, but the effect of sleeve gastrectomy (SG) on GERD remains controversial. The GERD-Health-Related Quality of Life (GERD-HRQL) questionnaire has been utilized as a reliable validated measure of GERD symptom severity for two decades and is a useful tool in assessing patient reported outcomes following conventional anti-reflux surgery. Objective: To determine the effects of RYGB and SG on GERD-HRQL.

Methods: Retrospective analysis of prospectively collected data was performed on 381 consecutive patients who underwent bariatric surgery between March 2015-January 2019. GERD-HRQL scores were measured preoperatively and at 6-months and 12-months post-operatively. Multivariate logistic regression and t-tests were used to compare patient GERD-HRQL changes from preoperative baseline. Results: $71.3 \%$ of patients received a RYGB; $29.7 \%$ received SG. RYGB patients had higher mean baseline GERD-HRQL scores: 8.67 for RYGB group, 4.76 for SG group $(\mathrm{p}=0.001)$. RYGB patients experienced a mean improvement of 4.8 points at 6 months postoperatively (95\% CI 3.85-6.4), compared to no significant change in the SG group (95\% CI -2.18-0.91). In spite of higher preoperative baseline scores, at 12 months postoperatively RYGB patients had significantly lower GERD-HRQL scores than SG, with mean scores of 3.54 for RYGB, 5.4 for SG $(p<0.001)$.

Conclusion: Gastric bypass is an effective treatment for GERD symptoms with sustained improvement in GERD-HRQL scores in our patient population. Sleeve gastrectomy did not significantly change symptoms of GERD.

\section{S157}

A structured early intervention program in patients with predicted poor long-term outcome following bariatric surgery: a prospective randomized study.

Panagiotis Drakos, MD; Panagiotis Volteas, MD; Kevin Seeras, DO; Shabana Humayon; Benjamin Flink, MD; Konstantinos Spaniolas, MD; Mark Talamini, MD; Aurora Pryor, MD; Stony Brook University.

Background: Early post-operative weight loss can be predictive of one-year outcomes. It is unclear if poor performers identified in the first post-operative month can have improvement in outcomes with additional support and education.

Purpose: Evaluate the impact of a structured targeted support program for patients with lower than average early post-operative weight loss on 1-year outcomes.

Method: This was a prospective randomized study of bariatric surgery patients who experienced less than 50th percentile excess body weight loss (\%EWL) at 3-weeks. Subjects with EWL $<18 \%$ were randomized into two groups: an intervention (IV) arm or a control (NI, no intervention) arm. The IV arm was offered a program with 7 weekly behavioral support sessions. NI patients received routine post-operative care. Results: 128 patients were randomized: 65 NI and 63 IV. In the IV group: 20 attended all sessions, 7 attended $<4,36$ did not participate. There was no difference in baseline demographics, procedure type, or BMI. At 1-year, there was no difference in \%EWL (ratio $0.993,95 \%$ CI $0.873,1.131$ ), \%EBMIL (ratio 0.997, 95\%CI $0.875,1.137$ ) and \%TWL (ratio $1.016,95 \%$ CI $0.901,1.146$ ) between groups. A subgroup analysis including only the subjects who participated in all seven sessions, showed similar results.

Conclusion: Patients who present with suboptimal weight loss early after bariatric surgery do not experience a significant weight loss improvement with a structured behavioral support program. Importantly, despite being alerted to their poor early weight loss, patients demonstrated poor adherence to the proposed intervention. 


\section{S158}

\section{Elevated Hemoglobin A1c level and Bariatric Surgery Complications}

Mahnoor Zia ${ }^{1}$; Ugoeze J Nwokedi ${ }^{2}$; Susan $\mathrm{Xu}^{2}$; Archana Sadhu $^{2}$; Bita Kash ${ }^{2}$; Vadim Sherman $^{2}$; Nabil Tariq ${ }^{2}$; 1Texas A\&M University; ${ }^{2}$ Houston Methodist Hospital.

Introduction: Literature in cardiac and orthopedic surgery suggests that elevated glycosylated hemoglobin (HbAlc) is a modifiable risk factor for postoperative complications. However, in bariatric surgery, there is insufficient evidence to assess the association of preoperative HbAlc with postoperative complications. Poor glycemic control is a major comorbidity in bariatric patients. The objective of this study was to assess the impact of $\mathrm{HbAlc}$, a measure of chronic glycemic control, on early postoperative outcomes in bariatric surgery patients.

Methods: Patients were selected from the Metabolic and Bariatric Surgery Accreditation and Quality Improvement Program (MBSAQIP) database. Between 2017 and 2018, 405,211 patients had undergone a bariatric surgery procedure. 140,601 patients had preoperative $\mathrm{HbAlc}$ recorded. Retrospective review was restricted to patients who had undergone Laparoscopic Sleeve Gastrectomy and Laparoscopic Roux-En-Y Gastric Bypass, excluding revisions, $n=118,742$ patients. Early post-operative complications were defined as those occurring within 30-days postoperative. Propensity score matching (PSM) using the nearest neighbor algorithm with one to one matching was used to balance differences between baseline demographic and clinical characteristics of patients between the groups defined by HbA1c with 31,554 patients. The complication rates before and after PSM were calculated and assessed by Fisher's exact test and conditional logistic regression, respectively. Analysis focused on data after PSM.

Results: At comparison group $\mathrm{A}, \mathrm{HbA} 1 \mathrm{c} \leq 8 \%$ versus $\mathrm{HbA} 1 \mathrm{c}>8 \%$, individual occurrence of superficial incisional, deep incisional and organ space infections did not have a significant difference. However, HbAlc $>8 \%$ was associated with higher incidence of any surgical site infection (SSI) when all SSIs combined $(0.95 \%$ vs. $0.69 \%$ P-value $=0.011)$. Readmission to intensive care unit $(1.06 \%$ vs. $0.82 \%, p=$ 0.032 ) and Emergency Department (ED) visits were more common with $\mathrm{HbAlc} \leq$ $8 \%(7.64 \%$ vs. $6.90 \%, \mathrm{p}=0.011)$. Comparison group $\mathrm{B}, \mathrm{HbA} 1 \mathrm{c} \leq 10 \%$ versus $\mathrm{HbA} 1 \mathrm{c}>10 \%$, showed a higher incidence of urinary tract infections $(0.42 \%$ vs $0.22 \%, \mathrm{p}=0.022)$, pulmonary emboli $(0.12 \%$ vs $0.02 \% \mathrm{p}=0.038)$, ED visits $(7.46 \%$ vs. $5.97 \%, \mathrm{p}<0.01)$, readmissions $(3.97 \%$ vs. $3.35 \%, \mathrm{p}=0.036)$, and interventions needed within 30 -days (Int30day) $(1.12 \%$ vs. $0.77 \% \mathrm{p}=0.021)$ at HbAlc $>10$. When comparing HbA1c $\leq 8 \%$ vs HbAlc $>10 \%$, ED visits $(7.18 \%$ vs $5.97, \mathrm{p}=$ $0.002)$ and Int30day $(1.13 \%$ vs $0.77 \%, p=0.016)$ were increased in HbAlc $\leq 8 \%$ while there was no difference in the other outcomes.

Conclusion(s): Elevated $\mathrm{HbAlc}$ is not associated with increased overall early postoperative complications in this large MBSAQIP dataset except when all SSI combined. Lower HbA1c is associated with some major adverse outcomes in this bariatric dataset. Further studies are needed to investigate these paradoxical findings.

\section{S159}

Complications Associated with Impaired Functional Health Status in Patients Undergoing Weight Loss Surgery: An Analysis of the Metabolic and Bariatric Surgery Accreditation Improvement Project (MBSAQIP)

Timothy Ritchie, BS; Zachary Wargel, BS; Andrew A Wheeler, MD; University of Missouri-Columbia.

INTRODUCTION: Weight loss surgery has been shown to be highly effective for losing weight and improving comorbid medical conditions. Patients with morbid obesity often suffer from impaired functional health and obesity can further hinder their activities of daily living (ADLs). Whether or not patients with impaired functional health represent a higher-risk group when having weight loss surgery is not known. Our aim is to understand the extent that impaired functional health status may independently contribute to postoperative complications in weight loss surgery patients.

METHODS: MBSAQIP patient use files, 2015-2018, were queried for all patients having undergone a primary laparoscopic Roux-enY gastric bypass (LRYGB) or laparoscopic sleeve gastrectomy (LSG). Complications were compared based on preoperative level of impairment of ADLs categorized as independent, partially dependent, or totally dependent. Patient characteristics were analyzed using Chisquare test to look at categorical variables. Logistic regression modeling was used to control for confounding variables. Significance was considered at $\mathrm{p}<0.05$.

RESULTS: 575,787 patients were identified who underwent primary LRYGB or LSG. After controlling for confounding variables, ADLs impairment was associated with increased risks of complications including: myocardial infarction, unplanned intubation, unplanned admission to ICU, and 30-day rates for death, readmission, reoperation, and intervention required.

CONCLUSION: LRYGB and LSG are safe procedures even when performed on patients who are have impaired ADLs. However, this group is at higher risk for complications and this must be considered when counseling these patients considering weight loss surgery. 
S160

\section{Post-Op Bariatric Patients During the Pandemic: Weight Loss} Falls off the Wagon

Rachel R Blitzer, MD; Arielle M Lee, MD; Estella Y Huang, MD; Jonathan Z Li, MD; Ryan C Broderick, MD; Bryan J Sandler, MD; Santiago Horgan, MD; Eduardo Grunvald, MD; Garth R Jacobsen, MD; UC San Diego.

Introduction: The coronavirus pandemic and measures to contain its spread have become a worldwide health and economic crisis. Bariatric patients are a particularly vulnerable population; they require extensive preoperative workup and management as well as close postoperative follow up, which may be less feasible under pandemic precautions. COVID-19 and isolation measures can impact many weight loss influences including stress, maintenance of routine, diet, substance use, mood disorders, and physical activity. This study assesses the pandemic's impact on weight loss in our pre- and postoperative bariatric patients.

Methods: A retrospective review of a prospectively maintained database identified patients who had undergone either sleeve gastrectomy (SG) or Roux-en-Y gastric bypass (RYGB) at a single institution from April 2019 - April 2020. Patients undergoing a medically supervised diet (MSD) were also identified; demographic and surgical data was obtained from both groups. Patient data were compared with institutional pre- and post- operative bariatric patient measurements prior to the pandemic. Patients were surveyed regarding pre- and post-COVID-19 weight measurement, dietary and physical activity habits, substance habits, and medical comorbidities.

Results: 184 patients were reachable by phone and surveyed; 127 patients were able to provide weight measurements from their home scale. Of these, 59 were undergoing MSD and 68 were postoperative bariatric patients (48 SG and 20 RYGB). Mean patient age was 43.7 years and mean body mass index (BMI) at last encounter was $40.7 \mathrm{~kg} / \mathrm{m} 2.85 .9 \%$ of patients were female. Average time from date of surgery to follow up call for post operative SG patients was 5.44 months (range $1.32-$ 31.23 months) and 7.53 months (range 2.23 - 19.16 months) for RYGB patients. SG weight loss rates were reduced from 12 to $3 \%$ EWL / month $(\mathrm{p}<0.001)$ while excess weight loss rates for RYGB patients decreased from 14 to $5 \%(\mathrm{p}<0.001)$. MSD patients had an insignificant reduction in EWL/month $(2.0 \%$ vs $1 \% \mathrm{p}=0.396)$ (Fig. 1). $60.9 \%$ of all patients endorsed decreased physical activity habits and $41.3 \%$ of patients stated their diet had worsened during COVID-19.

Conclusion: Social isolation measures during COVID-19 result in a significant reduction in weight loss rates for post-bariatric surgery patients. Medical weight loss patients did not experience similar effects, however all groups showed increased difficulty maintaining positive lifestyle modifications.

Figure 1: Excess Weight Loss Rates Pre- and Post Covid Restrictions

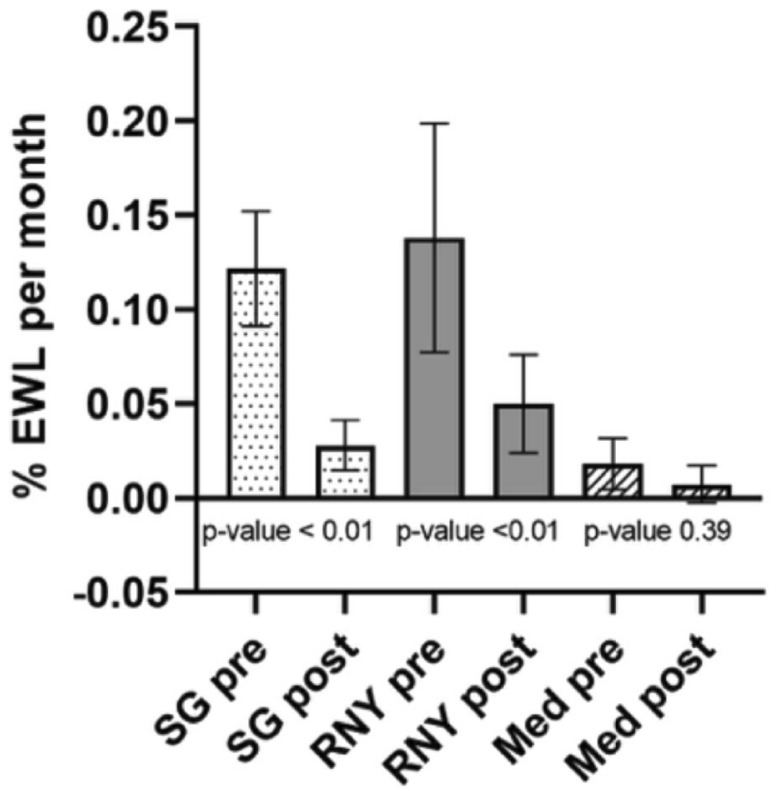

S161

Insulin-Like Growth Factor Binding Protein 2 Knockout Mice (Igfbp2-/-) Are Protected Against Trabecular Bone Loss After Vertical Sleeve Gastrectomy

Benjamin T Harris, $\mathrm{BSc}^{1}$; Phuong T Le, $\mathrm{MS}^{2}$; Alison Pletch, $\mathrm{MD}^{2}$; Clifford J Rosen, $\mathrm{MD}^{2} ;{ }^{1}$ University of New England College of Osteopathic Medicine; ${ }^{2}$ Maine Medical Center Research Institute.

Introduction: Bariatric surgery is a common procedure performed in the United States to treat obesity and its associated co-morbidities. It results in significant and sustained weight loss, as well as significant improvement in diabetes and cardiovascular disease. Likewise, unintended side effects of this surgery like accelerated and sustained bone loss are also not well understood. Insulin-like growth factor binding protein (IGFBP-2) may play a role in bone loss post vertical sleeve gastrectomy (VSG). In human studies at the Joslin Clinic, proteomics have revealed that IGFBP-2 is the most highly expressed protein in the serum within one week after VSG. In mice, VSG results in rapid depletion of bone and bone marrow adipose tissue has been associated with an increase in neutrophils and G-CSF in the circulation. Taken together, these data suggest there may be an acute inflammatory response in the marrow post VSG and that IGFBP-2 may play an early and essential role. It is unknown how VSG affects inflammation in Igfbp2 null mice (Igfbp2-/-), therefore, serum inflammatory markers such as IL-6, resistin, adiponectin, leptin and gene products of Sost, F4/80, Tnf-a, Il-6, Sema3E, and PlexinD1 were assessed. Methods: Twelve Igfbp $2+/+$ mice (control) and twelve Igfbp2-/- underwent either VSG or a sham surgery after 8 weeks on high fat diet. Whole femurs and blood serum were collected 4 weeks post-surgery. The serum was evaluated for IL- 6 , resistin, adiponectin, and leptin utilizing ELISAs. Whole femurs were analyzed for the gene expression levels of Sost, F4/80, Tnf-a, Il-6, Sema3E, and PlexinD1.

Results: A significant rise in serum IL-6 levels were observed post-operatively in the control VSG mice that was not seen in Igfbp2-/- mice $(p=0.0077)$. Additionally, resistin levels increased in the control mice after the sham surgery $(\mathrm{p}=0.0157)$. Conversely, resistin levels significantly decreased in Igfbp2-/- after surgery ( $\mathrm{p}=$ 0.0081 ). Serum levels of leptin and adiponectin significantly decreased after VSG ( $\mathrm{p}$ $=0.039$ in control mice for adiponectin; and $p=0.0224$ in control mice, $p=0.0036$ in Ifgbp2-/- for leptin). Surprisingly, gene expression did not produce any significant findings.

Conclusion: Bone loss in post-operative VSG patients has been associated with marrow inflammation. The blunted rise of IL-6 in the Ifgbp2-/- cohort and increased resistin levels in the control mice are indicative of an inflammatory response after VSG that is prevented in the Ifgbp2-/- mice. Thus, limiting circulating levels of IGFBP-2 in post-operative VSG patients may improve long term bone health. 
S162

\section{Underutilization of Roux-en-Y Gastric Bypass among Black Patients in the Metabolic and Bariatric Surgery Accreditation and Quality Improvement Program}

Benjamin J Flink, MD, MPH; Panagiotis Drakos, MD; Kevin Seeras, DO; Salvatore Docimo, DO, MS; Konstantinos Spaniolas, MD; Aurora D Pryor, MD, MBA; Stony Brook University.

Introduction: Among the two main surgical options for patients with obesity, Rouxen-Y gastric bypass (RYGB) is associated with greater weight loss and higher diabetes remission than sleeve gastrectomy (SG). We aim to assess for racial differences in use of RYGB compared to SG in the Metabolic and Bariatric Surgery Accreditation and Quality Improvement Program (MBSAQIP).

Methods: All patients with a BMI $>35$ undergoing non-emergent laparoscopic or robotic RYGB and SG in the MBSAQIP Participant User Files for 2015 to 2018 were included in the study. Patients were compared using Chi-squared, t-tests, and logistic regression.

Results: 603,469 patients were identified, of which $80 \%$ were female, $17.9 \%$ were Black, $13.8 \%$ were Hispanic, $73.8 \%$ had an ASA-class of 3 and the average BMI was 45.1. Overall, $28.9 \%$ of patients underwent RYGB, with the rate being significantly less for Black patients at $23.7 \%$ compared to $29.8 \%$ for White and $35 \%$ for Other race patients (all $\mathrm{p}<0.0001$ ). The significantly lower utilization of RYGB persisted on multivariate logistic regression accounting for preoperative patient characteristics (OR 0.7 [0.69-0.71], p < 0.0001).

Conclusions: RYGB is significantly underutilized compared to SG in Black patients, even when accounting for pre-operative patient characteristics. As a consequence of lower utilization of RYGB, Black patients may experience less weight loss and resolution of comorbidities. This study highlights the need for further investigation into the underlying cause of this racial disparity and to take steps to appropriately provide treatment to all patients regardless of race.

\section{S163}

Bariatric surgery and cardiovascular outcomes in patients with obesity and cardiovascular disease: A population-based cohort study

Aristithes G Doumouras, MD, $\mathrm{MPH}^{1}$; Yung Lee, $\mathrm{MD}^{1}$; Jorge Wong, MD, $\mathrm{MPH}^{1}$; Michael Paterson, $\mathrm{MSc}^{2}$; Branavan Sivapathasundaram, $\mathrm{MPH}^{1}$; Jean-Eric Tarride, $\mathrm{PhD}^{1}$; Lehana Thebane, $\mathrm{PhD}^{1}$; Dennis Hong, $\mathrm{MD}, \mathrm{MSc}^{1}$; Salim Yusuf, MBBS, DPhil ${ }^{1}$; Mehran Anvari, MD, $\mathrm{PhD}^{1} ;{ }^{1}$ McMaster University; ${ }^{2}$ University of Toronto.

Background: Bariatric surgery has been shown to significantly reduce cardiovascular risk factors. However, whether surgery can reduce major adverse cardiovascular events (MACE), especially in patients with established cardiovascular disease (CVD), remains poorly understood. We aim to determine the association between bariatric surgery and MACE among patients with cardiovascular disease and severe obesity.

Methods: This was a population-based, matched cohort study using province-wide multiple-linked administrative databases in Ontario, Canada. Patients with previous CVD who received bariatric surgery were matched on age, sex, heart failure history to controls from a primary care medical record database in a 1:1 ratio. Adjustments were made using the confounders such as patient socioeconomic status, comorbidities, smoking status, previous malignancy, cancer screening, healthcare utilization, and psychiatric history. The primary outcome was the incidence of extended MACE (first occurrence of all-cause mortality, myocardial infarction (MI), CABG/percutaneous coronary intervention, cerebrovascular events, and heart failure hospitalization). Secondary outcome included three-component MACE (MI, ischemic stroke, and mortality). Outcomes were evaluated through a combination of matching via propensity scores and subsequent multivariable adjustment

Results: 2,638 patients ( $n=1,319$ in each group) were included with a median follow-up time of 4.6 years. The primary outcome occurred in $11.5 \%$ of the surgery group and $19.6 \%$ of the controls (adjusted hazard ratio [HR] 0.58 ; $95 \%$ confidence interval $[\mathrm{CI}$, $0.48-0.71, \mathrm{P}<0.001)$. The observed effect was profound for those with heart failure (HR 0.44; 95\% CI, 0.31-0.62, $\mathrm{P}<0.001$; absolute risk reduction [ARR] $19.3 \%, 95 \% \mathrm{Cl}$ $12.0 \%-26.7 \%$ ). Those with ischemic heart disease also saw a substantial benefit (HR 0.60, $95 \%$ CI 0.48 to $0.74, \mathrm{P}<0.001$; ARR $7.5 \%, 95 \%$ CI $4.7 \%-10.5 \%$ ). Surgery also was associated with lower risk of the secondary outcome (HR $0.66,95 \% \mathrm{CI} 0.52-0.84, \mathrm{P}=$ 0.001 ) and cardiovascular mortality (HR $0.35,95 \% \mathrm{CI} 0.15-0.80, \mathrm{P}=0.001)$.

Conclusions: Bariatric surgery was associated with a large reduction in risk MACE in patients with CVD and obesity and requires confirmation by a large-scale randomized trial.

\section{S164}

Impact of Hiatal Hernia Repair Technique on Patient-Reported Gastroesophageal Reflux Symptoms following Laparoscopic Sleeve Gastrectomy

Anne P Ehlers, MD, MPH ${ }^{1}$; Aaron J Bonham, $\mathrm{MSc}^{1}$; Amir A Ghaferi, MD, MS ${ }^{1}$; Jonathan F Finks, MD ${ }^{1}$; Arthur M Carlin, MD ${ }^{2}$; Oliver A Varban, MD ${ }^{1} ;{ }^{1}$ University of Michigan; ${ }^{2}$ Henry Ford Health System.

Introduction: Repairing a hiatal hernia at the time of laparoscopic sleeve gastrectomy (LSG) may help resolve pre-existing symptoms of gastroesophageal reflux disease (GERD) or reduce the risk for de novo GERD symptoms. However, different hiatal hernia repair techniques exist and their impact on symptom control is unclear. Methods and Procedures: Surgeons $(n=75)$ participating in a statewide quality collaborative were surveyed on their technique for repair of hiatal hernias during LSG. Options included anterior repair (AR), posterior repair (PR) and posterior repair with mesh (PRM). We used the GERD health-related quality of life (GERDHRQL) survey to evaluate GERD symptoms at baseline and one year following LSG among patients who underwent concurrent hiatal hernia repair. We compared patient-reported GERD-HRQL symptom scores, total weight loss at one year, and 30-day complications among patients according to their surgeon's operative technique (AR $n=866$ patients, $P R n=6,131$ patients, $P R M n=886$ patients).

Results: According to surgeon surveys, the most commonly reported technique for hiatal hernia repair was PR $(n=64,85.3 \%)$, followed by PRM $(n=7,9.3 \%)$ and AR $(n=4$, $5.3 \%$ ). Patients who underwent LSG by surgeons who perform AR were less likely to have a baseline diagnoses of GERD (AR 55.3\%, PR 59.5\%, PRM 64.8\%, p <0.01), and were modestly more likely to experience worsening GERD symptoms at one year (AR $29.8 \%$, PR $28.7 \%$, PRM $28.2 \%, \mathrm{p}<0.0001$ ), despite experiencing similar total body weight loss (AR $29.8 \%$, PR $28.7 \%$, PRM $28.2 \%, \mathrm{p}=0.08$ ). There was no significant difference in satisfaction rates with GERD symptoms at one year (AR 73.2\%, PR 76.3\%, PRM 75.7\%, p = 0.43), and risk-adjusted 30-day rates of hemorrhage or leak were similar among all groups.

Conclusions: Patients undergoing LSG with concurrent hiatal hernia repair by surgeons who typically perform an anterior repair of a hiatal hernia were somewhat more likely to report worsening GERD at one year after surgery despite excellent weight loss. However, overall satisfaction with GERD symptoms after LSG is high, regardless of technique, indicating that symptom control for GERD may be multifactorial.

\section{S165}

Title: Re-Sleeve Gastrectomy for Failed Primary Laparoscopic Sleeve Gastrectomy: A Single Institution Case Series.

Jean B Guerrier, $\mathrm{MD}^{1}$; Erica Amianda, $\mathrm{PA}^{1}$; Thomas Gavigan, $\mathrm{MD}^{2}$; Douglas Ewing, MD ${ }^{1}$; Hans Schmidt, MD ${ }^{1}$; Sebastian Eid, $\mathrm{MD}^{1}$; ${ }^{1}$ Hackensack University Medical Center; ${ }^{2}$ Atrium Health-Carolinas Medical Center.

Background: Laparoscopic sleeve gastrectomy (LSG) is associated with excellent weight loss, low morbidity and mortality. However, weight regain or inadequate weight loss with resulting recurrence of comorbidities after LSG is not uncommon in the long term. Laparoscopic revisional sleeve gastrectomy or laparoscopic re-sleeve gastrectomy (LRSG) is gaining popularity to address this problem.

Objectives: The purpose of this study is to report the outcomes of revision sleeve gastrectomy at a single academic institution.

Method: Retrospective review of a single-center prospectively maintained database. All the patients who underwent LRSG between August 2017 and September 2019 for weight regain or inadequate weight loss were included. Preoperative patient's demographics, comorbidities, and volumetric studies if performed, excess weight loss percentage, were recorded. Primary outcome include excess weight loss 6 months, 1 year, and 2 years after surgery. Secondary outcomes include serious complications, and reoperation.

Results: Twenty-one patients underwent LRSG during the period examined. The mean weight at the time of LRSG was $126 \mathrm{~kg}$ (BMI of 42.7). Ninety percent of LRSG were performed for inadequate weight loss, and $80 \%$ of those patients who had a CT volumetric study of their stomach demonstrated volumes greater than $500 \mathrm{cc}$. The median change in BMI after LRSG was $7.1(3.3-7.8)$ and median $\%$ EWL was $27.2(20.3-48.6)$. The post-operative BMI $(\mathrm{M}=37.2, \mathrm{SD}=8.6)$ was not significantly lower than the preoperative BMI $(M=44.7, \mathrm{SD}=9.8) ; \mathrm{t}(20)=0.22$, $\mathrm{p}=0.83$. Nine patients $(43 \%)$ had major complications, and $24 \%$ required a reoperation.

Conclusion: LRSG is an acceptable option for weight loss failure after primary sleeve gastrectomy for a few, carefully selected patients. Additional, prospective studies are needed to prove its long-term efficacy. 
S168

Enhancing robotic efficiency through the eyes of robotic surgeons

Courtney A Green, MAEd, MD ${ }^{1}$; Joseph Lin, MD ${ }^{2}$; Emily Huang, MAEd, MD 3 ; Patricia O'Sullivan, EdD ${ }^{2}$; Rana Higgins, $\mathrm{MD}^{4}$; ${ }^{1}$ Mayo Clinic; ${ }^{2}$ University of California San Francisco; ${ }^{3}$ Ohio State University Medical Center; ${ }^{4}$ Medical College of Wisconsin.

Introduction: Robotic technology affords surgeons many novel features-unfortunately the exponential decision tree can easily overwhelm operators and distract from case progression. Throughout the popularization of robotic surgery, two stereotypes have prevailed: robotic surgery is expensive and inefficient. Through repetition and expertise, data suggests surgeons can overcome these challenges, allowing efficient utilization of this technology to improve patient outcomes and decrease economic burden. In an effort to identify educational opportunities and improve operative efficiency, we performed a sub-analysis of expert observation of robotic surgery.

Methods: Expert robotic surgeons, identified through high case volumes and contributions to the surgical literature, reviewed 8 anonymous video clips portraying key portions of two robotic general surgery procedures. While watching, surgeons commented on what they saw on the screen. All interactions with participants were in person, recorded, transcribed, and analyzed at a later date. Prior to watching the videos, surgeons completed a demographic questionnaire. Using content analysis, four researchers double-coded each transcript applying a consensus developed codebook.

Results: Seventeen surgeons, from 13 different US states, participated. The average participant was male $(82.4 \%), 46.76(\mathrm{SD}=6.64)$ years old, had $13.2(\mathrm{SD}=8.23)$ years of teaching experience, worked in urban academic hospitals $(64.7 \%)$ and performed $643(\mathrm{SD}=467)$ robotic operations at the time of interviews working with multiple levels of trainees using robotic technology (64.7\%). Emphasis on efficiency (or lack thereof) surfaced across three main themes: overall case progression, robotic capabilities and instrumentation. Regarding progression, experts verbally rewarded purposeful and "ergonomically sound" movements. Alternatively, language such as "flirting," "digging," or "playing with the tissue," reflected impatience with repetitive and indecisive movements, which was attributed to presumed inexperience. Efficiency of robotic capabilities included enhanced visualization, use of the third arm for improved exposure and the wristed components compared to the "straight stick" approach. Compared to laparoscopy, experts cited these features resulting in "a much more delicate, precise operation. Just ... just way more elegant." Finally, experts discussed instrumentation choice, highlighting limitations and versatility with bipolar and monopolar energy.

Conclusions: Improving efficiency is critical to optimizing robotic technology; surgeons must navigate the operation itself while simultaneously managing multiple platform components and instrumentation features. This study highlights three areas for improved efficiency: case progression, robotic capabilities and instrumentation. Development of education materials that focus on these themes could help robotic surgeons and surgical educators overcome one of robotic technology's persistent challenges.

\section{S169}

\section{Laparoscopic vs Robotic Inguinal Hernia Repair: A Single- Center, Case-Matched Study}

Sullivan A Ayuso; Bola G Aladegbami, MD; Tanu Prasad, MA; Vedra A Augenstein, MD; Carolinas Medical Center.

Introduction: Robotic inguinal hernia repair (RIHR) is gaining popularity and is the minimally invasive alternative to laparoscopic inguinal hernia repair (LIHR). Thus far, there is little data directly comparing LIHR and RIHR. The purpose of this study will be to compare outcomes for LIHR and RIHR at a single center.

Methods: A prospective institutional hernia database was queried for patients who underwent LIHR or RIHR from 1999 - 2020. The patients were then matched based on the surgeon performing the operation and unilateral vs bilateral repair. Standard descriptive statistics were used, and a univariate analysis was performed between the LIHR and RIHR groups.

Results: There were 236 patients who met criteria for the study, 118 LIHR and 118 RIHR; 24 patients $(20.3 \%)$ in each group had a bilateral repair. LIHR patients were slightly younger $(54.4 \pm 15.6$ vs $58.6 \pm 13.8 ; \mathrm{p}=0.03)$ but similar in terms of BMI $(27.1 \pm 5.1$ vs $29.1 \pm 2.1 ; \mathrm{p}=0.70)$ and number of comorbidities $(2.9 \pm 2.5$ vs $2.6 \pm$ $2.2 ; \mathrm{p}=0.59)$. All patients had a CDC $1(99.3 \%$ vs $98.6 \%)$ or CDC $2(0.7 \%$ and $1.4 \%$ ) wound class. Synthetic mesh was placed in every case, and there were no intraoperative complications. Operative time was not found to be significantly different between the two groups $(109.1 \pm 50.0$ vs $100.6 \pm 38.3 \mathrm{~min} ; \mathrm{p}=0.36)$. Postoperative urinary retention was similar $(7.8 \% \mathrm{vs} 5.7 \% ; \mathrm{p}=0.59)$. There was only one wound infection, which was in the robotic group. There were a comparable number of seromas $(6.6 \%$ vs $8.1 \% ; \mathrm{p}=0.65)$ but none required intervention. No patients required return to the operating room for complications relating to their surgery. There were five hospital admissions following the operation ( $1.4 \%$ vs $2.1 \%$; $\mathrm{p}=0.68$ ), no 30-day readmissions were in the LIHR group and three 30-day readmissions in the RIHR group $(\mathrm{p}=0.28)$. One of the readmissions was for wound infection, one for a bowel obstruction, and another for a rash related to the prep that was used. Recurrence rates were low for both groups $(0.7 \%$ vs $1.4 \% ; \mathrm{p}=0.38)$ with a mean follow-up time $13.0 \pm 13.3$ months.

Conclusions: LIHR and RIHR are both performed with low morbidity and have comparable overall outcomes. Operative time was comparable between the groups. Either LIHR or RIHR may be considered when performing inguinal hernia repair and should depend on surgeon and patient preference.

\section{S170}

\section{A comparison of laparoscopic and robotic ergonomic risk: pick your poison}

Luke Umana, $\mathrm{MD}^{1}$; Sara Monfared, $\mathrm{MD}^{1}$; Dimitrios Athanasiadis, $\mathrm{MD}^{1}$; Hamed Asadi, $\mathrm{MS}^{2}$; Cameron Colgate, $\mathrm{MS}^{1}$; Denny $\mathrm{Yu}, \mathrm{PhD}^{2}$; Dimitrios Stefanidis, MD, $\mathrm{PhD}^{1} ;{ }^{1}$ Indiana University School of Medicine; ${ }^{2}$ Purdue University.

INTRODUCTION: Work related injuries in minimally invasive surgery (MIS) are common because of the strains placed on the surgeon's or assistant's body by the technology interface. The objective of this study was to compare surgeon ergonomic risk between robotic and laparoscopic surgery.

METHODS AND PROCEDURES: Objective and subjective ergonomic data were recorded from surgeons and surgical trainees during robotic and laparoscopic procedures. Perceived discomfort questionnaires were obtained before and after each operation. Intraoperatively, biomechanical loads were objectively captured using motion tracking sensors and electromyography (EMG) sensors. Perceived discomfort, body position and muscle activity were compared between robotic and laparoscopic procedures using a linear regression model.

RESULTS: Ergonomic data were recorded from 20 surgeons and surgical assistants participating in 29 robotic and 49 laparoscopic procedures. For laparoscopic cases we identified a significant postoperative increase in right finger numbness $(p=0.073)$ and right shoulder stiffness $(p=0.018)$. For robotic cases we found an increase in back stiffness $(p=0.046)$. Linear regression analysis demonstrated robotic surgery led to increased right and left hand/shoulder pains (coefficient $0.8 ; p=0.032$ and $0.22 ; \mathrm{p}<0.001$, respectively) but lower right deltoid $(-1.68 ; \mathrm{p}=0.008)$ and right trapezium $(-2.18 ; \mathrm{p}=0.001)$ muscle activity recordings. No differences were seen in the respective muscle groups of the left side. Demanding and static positioning were similar between the two methods except for more frequent static neck positions recorded during robotic operations $(7.73 \mathrm{p}=0.014)$.

CONCLUSION: Both the laparoscopic and robotic techniques are associated with ergonomic risk but affect different muscle groups. Given the frequency of musculoskeletal injuries among MIS surgeons this knowledge can help develop programs of ergonomic risk prevention that may increase their longevity. 


\section{S171}

Multimodal Mapping for Uro-Oncology Robotic Surgery: Fusing Rapid Evaporative Ionisation Mass Spectrometry (REIMS) with freehand 3D Ultrasound Imaging in a Robust Tracking Framework

Erik Mayer; Philip Pratt; Eftychios Manoli; Afeez Adebesin; Petra Paizs; James Kinross; Justin Vale; Zoltan Takats; Ara Darzi; Imperial College London.

Aim To develop a single technology platform combining real-time freehand 3D ultrasound-based intraoperative image guidance with intelligent Knife (iKnife) tissue diagnostics, delivering contemporaneous 'disease maps' that could be leveraged by autonomous surgical robotic systems.

Methods 1) Instrument and hardware development: Computer Aided Designs for a grasper attachment were 3D-printed and integrated with the Intuitive Surgical da Vinci Cadiere forceps. This was wrapped around an existing iKnife electrocautery tip (Fig. 1). The surgical aerosols were aspirated into the Xevo G2-XS QToF mass spectrometer (Waters Corporation). 2) Algorithm development: Vision-based tracking and kinematic chain updates were integrated via the da Vinci API; the software supporting semi-automated hand-pattern calibration for estimating the spatial relationship between the frame defined by the tracking pattern and the most distal frame of the robot arm (hybrid tracking framework). The OpenIGTLink interface was used for displaying REIMS tissue classifications. 3) Clinical feasibility testing: Spectral data were collected from four human radical nephrectomy cases for cancer resulting in a total of 115 samples. The relative abundance of cellular metabolites in the $\mathrm{m} / \mathrm{z}$ range of 150-1000 was subjected to univariate (ANOVA) and multivariate statistical analyses including principle components analysis and orthogonal partial least squares discriminant analysis.

Results Successful end-to-end system integration tests using animal tissue phantoms (porcine/chicken liver) demonstrated the applicability of the combined systems, with data visualisation displaying tissue classification events (Fig. 2).

The human renal samples were analysed and the environment of sampling points was examined resulting in 538 histologically annotated spectra (Capsule $=17$; Cortex $=$ $67 ;$ Medulla $=120 ; \mathrm{PF}=91 ; \mathrm{PUJ}=46 ;$ Tumour $=189)$. The OPLS-DA model (Fig. 3 ) showed significant separation between disease and non-disease samples (R2Y = 0.931; Q2Y $=0.763$ ) based on the different metabolic profiles observed.

A novel phantom kidney was made using porcine kidney with tumours fashioned from ballistic gel and incorporating two different types of gelatine (porcine and vegetable-based), melted onto the surface so as to be almost invisible to the naked eye, to simulate positive margins left behind after simulated tumour resection (Fig. 4). This will assess, in the laboratory setting, a surgeon's ability to perform an accurate 3D-ultrasound scan, followed by careful surveillance of the resulting defect by means of iKnife tissue classification.

Conclusion This feasibility study has successfully fused REIMS technology with $3 \mathrm{D}$-volumetric imaging and a surgical robot to create a tissue sampling platform and has demonstrated the diagnostic precision of robotic REIMS in human renal tissues. Ongoing work is developing autonomous operation under computer control with input from the iKnife and the endoscopic camera.

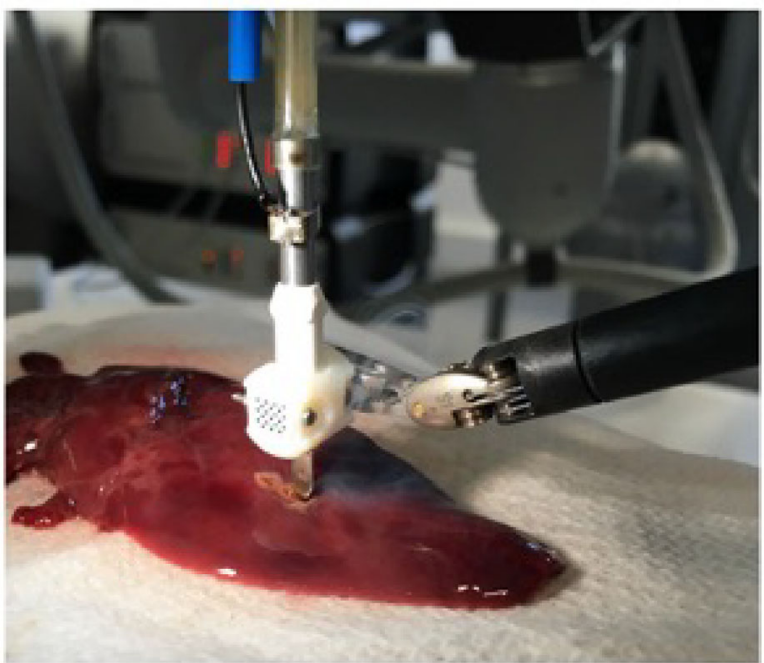

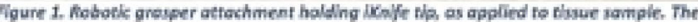
imake plume can be iesun to the fight of the firive turn uiter.

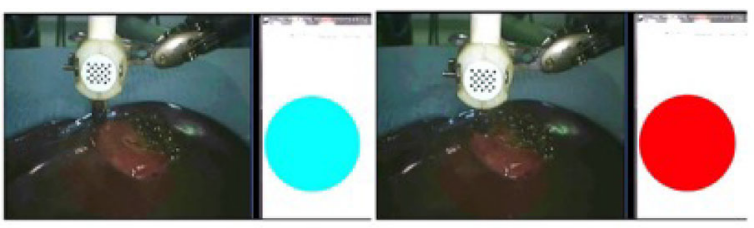

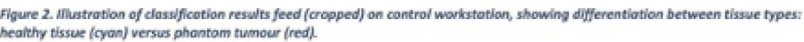

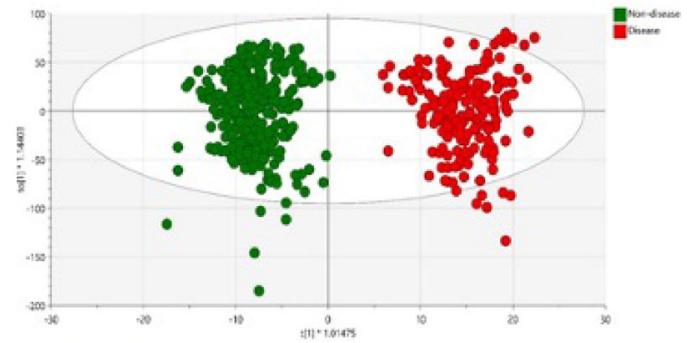

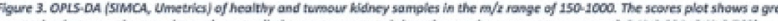

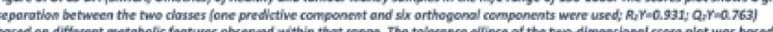

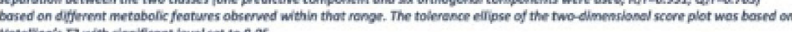
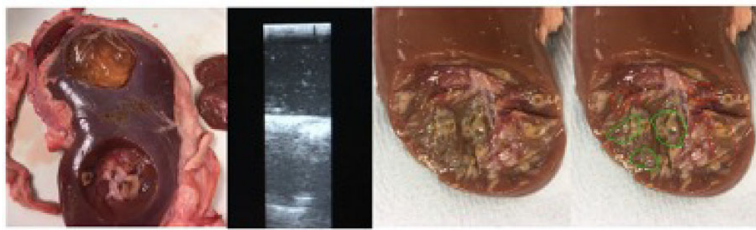

Figure 4. Parcine kildney with balibstic gel tumour phantam (left). Resectian defect with phantom positive margins added using poreine (red) and wegetable-based (green) gelatine (middile right and right). Uitrasound image ocqulred with the 30 inger orefloy for the simulated resection of tumour (middie left). 
S172

Use of humanoid robots and tablets to increase the efficiency of informed consent in Ivor-Lewis esophagectomy-a prospective randomized study

Rabi R Datta, MD; Justus Toader, MD; Dolores T Müller, MD; Thomas Dratsch; Celena Kazikci; Sabrina Turanli; Julia Peschka; Alexander R Stier; Matthias Scholz; Isabell Wahler, MD; Benjamin Babic, MD; Wolfgang Schröder, MD; Christiane J Bruns, MD; Hans F Fuchs, MD; University of Cologne, Department for General, Visceral, Cancer and Transplant Surgery.

Introduction: Telemedicine includes a variety of novel technologies and is becoming more and more essential in the clinical routine. The term telemedicine relates to the implementation of telecommunication technology in the context of medical care. This includes virtual or robotic assisted patient contact and may lead to remote-controlled surgical operations. Particularly in times of the SARS-CoV-2 pandemic, there was an urgent need for a reduction of live patient contact while still offering the best possible medical therapy in order to interrupt infection chains. Preoperative patient counseling is indispensable. In order to make informed consent as effective as possible, it can be useful to provide the patient with additional information using humanoid robots or tablets.

Aim of the study: The aim of this study was to investigate how useful a preoperative information of patients by a humanoid robot (Pepper) is compared to information with a tablet only.

Methods: The study was performed at the University Hospital of Cologne as part of regular preoperative counseling before esophagectomy with gastric pull-up (IvorLewis). After randomization, patients received a medical briefing either with a tablet or the humanoid robot before counseling was performed by the doctor. The information provided was identical in both cases. Patients evaluated their experience with a questionnaire. In addition, patients answered 11 multiple-choice questions in order to measure the information given by the tablet and the robot.

Results: A total of 28 patients ( 22 male, 6 female; mean age: 65.0 , range: $47.0-80.0$ ) participated in the study. Fifteen patients completed informed consent using the tablet, 13 patients using the humanoid robot. There were no significant differences between the informed consent with the tablet or the robot. There was no significant difference in satisfaction regarding informed consent $(p=0.301)$ and preoperative anxiety $(\mathrm{p}=0.649)$. The knowledge test also showed no significant differences between informed consent using the tablet (mean score: $78.7 \%$ ) or the robot (mean score: $75.5 \%)(\mathrm{p}=0.558)$.

Conclusion: Support of a medical briefing using humanoid robots or tablet computers prior to an Ivor-Lewis esophagectomy was very well received in our clinic by patients and staff. Additional information transfer by humanoid robots and tablets may be possible. Measures do not differ between the two compared machines. New technologies should be more routinely utilized to make informed consent more effective.

\section{S173}

The evolution of the general surgery resident operative case experience in the era of robotic surgery

Nnenna S Nwaelugo; Matthew I Goldblatt, MD; Jon C Gould, MD; Rana M Higgins, MD; Medical College of Wisconsin.

Introduction: As robotic-assisted surgical procedures increase, more general surgery residency programs need established robotic training curricula. In this era of minimally invasive surgery, concerns exist regarding the impact of newer technologies on the residents' operative case distribution, specifically laparoscopic and open surgery. The objective of this study was to analyze the impact of a growing robotic operative case volume and established robotic surgery training curriculum on resident operative case experience.

Methods: The robotic surgery training curriculum at a single academic institution was established in 2017. Resident ACGME operative case logs from academic years (AY) 2014 to 2020 were analyzed to determine participation in open, laparoscopic and robotic cases for three years prior to and three years after institution of the curriculum. Case categories included were alimentary tract, abdomen, endocrine, thoracic, pediatric and trauma. Overall cases, as well as participation by case type, post-graduate year (PGY) level, resident role and institution type were analyzed. A one-way analysis of variance (ANOVA) was used to analyze differences within and across each category throughout the six academic years.

Results: Operative case logs from 77 residents were analyzed, with a total of 34,757 overall cases: 20,604 (59.3\%) open, 13,768 (39.6\%) laparoscopic and $385(1.1 \%)$ robotic. Between 2014 and 2020, there was no significant change in the residents' number of open or laparoscopic cases. However, there was a significant increase in robotic cases, from $18(0.3 \%)$ overall cases in the 2014-15 AY, to $201(3.7 \%)$ in the 2019-20 AY ( $\mathrm{p}=0.01$ ) (Fig. 1). The operative case categories with significan increases in robotic cases were foregut $(1.1 \%$ to $5.1 \%, \mathrm{p}=0.01)$, intestinal $(0.2 \%$ to $1.8 \%, \mathrm{p}=0.03)$ and hernia $(0.3 \%$ to $8.6 \%, \mathrm{p}=0.003)$. There was a significant increase in the number of residents' robotic cases at academic $(0.2 \%$ to $3.0 \%, \mathrm{p}=$ $0.01)$ and veterans' hospital $(0.0 \%$ to $2.0 \%, \mathrm{p}=0.01)$ institutions. The resident role with a significant increase in robotic cases was first assistant $(1.8 \%$ to $9.8 \%, \mathrm{p}=$ 0.004), with no changes as a surgeon junior or chief. There was no significant difference across PGY levels by surgical approach.

Conclusion: This study highlights the effectiveness of a standardized robotic surgery training curriculum at increasing resident participation in robotic cases without detrimental effects on participation in open and laparoscopic cases. As robotic cases continue to increase, the impact on laparoscopic and open case volumes needs to be carefully monitored to ensure a well-balanced resident training experience.
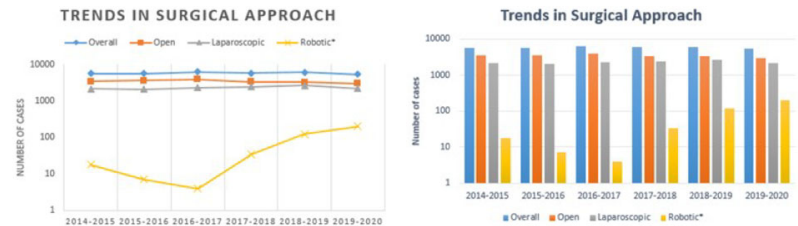

Figure 1. Trends in surgical approach (open, laparoscopic and robotic) across academic years 2014-2020. * (Asterisk) represents $p<0.05$. 
S174

\section{Robotic Stray Energy with Constant Voltage versus Constant Power Regulating Electrosurgical Generators}

Krzysztof J Wikiel, MD; Teresa S Jones, MD; Franklin J Powlan; Thomas N Robinson, MD; Edward L Jones, MD; University of Colorado.

Introduction: Stray energy from surgical energy instruments can cause unintended thermal injuries. There are no published data regarding electrosurgical generators and their influence on stray energy transfer during robotic surgery. There are two approved generators for the DaVinci Xi robotic platform: a constant-voltage regulating generator (cVRG) and a constant-power regulating generator (cPRG). The purpose of this study was to quantify and compare stray energy transfer in the robotic $\mathrm{Xi}$ platform using a cVRG versus a cPRG.

Methods: An ex vivo bovine model was used to simulate a standard multiport robotic surgery. The DaVinci Xi (Intuitive Surgical, Sunnyvale, CA) robotic platform was attached to a trainer box using robotic ports. A $5 \mathrm{~s}$, open air activation of the monopolar scissors was done at various settings using a cPRG (ForceTriad, Covidien-Medtronic, Boulder, CO) and cVRG (ERBE VIO dV 2.0, ERBE USA, Marietta, GA). Experiments were repeated 10 times similar to previous studies. Stray energy transfer was quantified with a thermal camera as temperature change of the tissue $\left({ }^{\circ} \mathrm{C}\right.$ ) adjacent to the tip of the assistant grasper (which was not in direct contact with the active monopolar scissors).

Results: Stray energy transfer occurred with both generators. Utilizing common, comparable settings for standard coagulation, less stray energy was transferred with the cVRG versus cPRG $\left(4.4 \pm 1.6^{\circ} \mathrm{C}\right.$ vs. $\left.41.1 \pm 13.0^{\circ} \mathrm{C}, \mathrm{p}<0.001\right)$. Similarly, less stray energy was transferred using cut modes with the cVRG compared to the cPRG $\left(10.0 \pm 12.3^{\circ} \mathrm{C}\right.$ vs. $\left.33.9 \pm 18.4{ }^{\circ} \mathrm{C}, \mathrm{p}<0.001\right)$. This trend persisted even at high coagulation settings with the cVRG increasing temperatures less than the cPRG $\left(15.8 \pm 7.7^{\circ} \mathrm{C}\right.$ vs. $\left.45.8 \pm 22.2^{\circ} \mathrm{C}, \mathrm{p}<0.001\right)$.

Conclusions: Stray energy transfer occurs during surgery with the DaVinci Xi robotic platform. This stray energy can increase tissue temperature more than $45^{\circ} \mathrm{C}$ and could result in unintended burn or tissue necrosis. A cVRG generator, on average, transfers nearly $10 \times$ less stray energy than a cPRG on comparable coagulation mode settings. Similar reductions in stray energy transfer are seen in other comparable energy mode outputs.

\section{S175}

\section{Impact of BMI on Outcomes in Veterans Undergoing Robotic} Inguinal Hernia repair

Justine O Chinn, $\mathrm{BS}^{1}$; Rene Telez, $\mathrm{MD}^{2}$; Bunchhin Huy, $\mathrm{BS}^{3}$; Cyrus Farzaneh, $\mathrm{MD}^{2}$; Ashton Christian, $\mathrm{MD}^{2}$; Jay Ramsay, $\mathrm{BS}^{1}$; Hubert Kim, $\mathrm{MD}^{4}$; Marcelo Hinojosa, $\mathrm{MD}^{2}$; Brian $\mathrm{R}$ Smith, $\mathrm{MD}^{2} ;{ }^{1}$ University of California, Irvine School of Medicine; ${ }^{2}$ University of California, Department of Surgery; ${ }^{3}$ University of California, Irvine; ${ }^{4}$ Veterans Affairs Long Beach Healthcare System.

Introduction: Body mass index (BMI) is a significant risk factor for recurrence and post-operative complications of both open and laparoscopic inguinal hernia repair. Robotic-assisted inguinal hernia repair (RIHR) has been demonstrated to be a safe and viable option for inguinal hernia repair. The objective of this study is to determine how BMI influences operative times, clinical outcomes, and recurrence after RIHR.

Methods: We performed a retrospective review of patients who underwent RIHR between 2012 and 2019. The operating times, clinical outcomes, recurrence rate, and morbidity for RIHR were compared between three BMI Groups. Patients were divided into normal weight (BMI $<25$ ), overweight (BMI 25-29.9), and obese (BMI $>30$ ). Analysis was performed using pairwise comparisons t-test with Benjamini \& Hochberg adjustment method and Pearson's Chi-square test with Yates' continuity correction.

Results: A total of 304 patients underwent RIHR, of which 102 patients were normal weight, 120 were overweight, and 82 were obese. Of these patients, $99 \%$ were male, and the mean age was 59. Demographics and comorbidities did not differ significantly, except for a higher rate of smoking in the normal weight group when compared to overweight and obese groups $(\mathrm{p}<0.05)$. For bilateral inguinal hernia operating time was significantly lower in the normal weight group $(83.5 \mathrm{~min})$ compared to the overweight group $(98.4 \mathrm{~min})(\mathrm{p}<0.01)$ and obese group $(97.8 \mathrm{~min})$ $(\mathrm{p}<0.01)$. For unilateral repair, the average operating time by group was significantly longer in the obese group $(85.6 \mathrm{~min})(\mathrm{p}<0.01)$ compared to both the normal weight group $(65.2 \mathrm{~min})$ as well as the overweight weight group $(70.9 \mathrm{~min})(\mathrm{p}=$ 0.01 ). There was no significant difference in clinical outcomes including reoperation, recurrence, readmission, and overall morbidity and mortality between BMI groups. Conclusion: Operative times in overweight and obese patient undergoing RIHR were significantly longer than that of normal weight patients. Despite these increased operative times, there was no difference in postoperative complications or recurrence in the higher BMI patient when compared to the normal weight patients. Although obesity increases the risk for recurrence and post-operative complications in hernia repairs, our results suggest that the robotic approach may mitigate these risks. 


\section{IMPLICATIONS OF NEAR-INFRARED IMAGING AND INDOCYANINE-GREEN ON OUTCOMES FOLLOWING ELECTIVE COLORECTAL SURGERY: A SYSTEMATIC REVIEW AND META-ANALYSIS}

Kevin K Verhoeff, MD; Valentin Mocanu, MD; Breanna Fang; Jerry Dang, MD; Janice Y Kung, MLIS; Noah J Switzer, MD, MPH; Daniel W Birch, MD, MSc; Shahzeer Karmali, MD, MPH; University of Alberta.

Introduction: Anastomotic leaks are amongst the most feared complications following colorectal surgery with rates remaining high despite advances in surgical technique and perioperative care. Indocyanine green (ICG) angiography is a costeffective, safe, and readily available technology enabling intraoperative assessment of anastomotic perfusion. However, a paucity of comprehensive reviews currently exists precluding consensus regarding its utilization.

Methods and Procedures: A comprehensive search of MEDLINE, Embase, Scopus, and Web of Science databases was conducted on July 2020 by a medical librarian. Studies were reviewed and data extracted independently by two reviewers following PRISMA guidelines. Inclusion criteria were ICG use in colorectal surgery, studies with comparison groups, and studies with $>5$ patients. Abstracts, non-English, and pediatric studies were excluded. Our primary outcome was evaluating anastomotic leaks with and without ICG. Secondary outcomes included differences in intraoperative anastomotic revisions, ureteric injuries, reoperations, length of stay, and operative time.

Results: A total of 1586 studies were retrieved with 26 meeting final inclusion criteria ( 2 randomized control trials; 2 prospective cohorts; 21 retrospective cohorts, and 1 retrospective case control). Included studies comprised 7888 patients of which $3587(45.5 \%)$ received ICG. No differences in basic demographics were observed between ICG and control cohorts with respect to age (63.6 vs 64.0 years; $p=0.98)$, sex $(41.0 \%$ vs $43.4 \%$ female; $p=0.73)$, smoking status $(22.5 \%$ vs $24.2 \%$ active smokers, $\mathrm{p}=0.81)$, or diabetes $(13.8 \%$ vs $14.1 \% ; \mathrm{p}=0.96)$. Technical factors such as anastomotic distance from the anal verge $(8.0 \mathrm{~cm}$ vs $8.3 \mathrm{~cm} ; \mathrm{p}=0.37)$, diversion $(55.5 \%$ vs $58.9 \% ; \mathrm{p}=0.58)$, and anastomotic technique $(78.7 \%$ stapled vs $75.0 \%$ stapled; $\mathrm{p}=0.59$ ) were not different between ICG and controls respectively. Metaanalysis demonstrated that ICG was associated with reduced odds of anastomotic leak (OR 0.42 ; 95\% CI $0.34-0.53 ; \mathrm{p}<0.001$ ), an effect that was more pronounced in subgroup analysis of rectal cancer studies (OR $0.34,95 \%$ CI $0.24-0.48 ; p<0.001$ ). No differences in reoperation rates, length of stay, or operative time were observed between groups. Other secondary outcomes such as ureteric injury and long-term complications could not be evaluated due to study heterogeneity.

Conclusions: ICG presents a promising strategy to address anastomotic leaks, yet ongoing prospective randomized controlled trials which characterize the full spectrum of complications are needed to further delineate the role of routine ICG use in colorectal surgery.
Quadruple purse-string suture for single stapling technique following transanal total mesorectal excision for rectal cancer.

Shinichiro Mori; Yoshiaki Kita; Masumi Wada; Takako Tanaka; Soichi Satake; Ken Sasaki; Takaaki Arigami; Hiroshi Kurahara; Takao Ohtsuka; Department of Digestive Surgery, Breast and Thyroid Surgery Graduate School of Medicine, Kagoshima University.

Purpose: The aim of this study was to compare the completeness of resected rings of the quadruple purse-string sutures (PSS) and the double PSS for single stapling technique (SST) following transanal total mesorectal excision (taTME) for rectal cancer.

Methods: Consecutive patients who underwent taTME with SST for low rectal cancer from November 2014 to October 2020 were enrolled. Primary outcome was the rate of completeness of resected rings. The technique was evaluated in terms of the full thickness of the resected ring of the colonic and the rectal stump. We also assessed intraoperative adverse events, blood loss, anastomotic leakage and the other short-term outcomes, length of hospital stay, quality of TME. Quadruple PSS consisted of double PSS for the colonic stump and double PSS for the rectal stump. Double PSS consisted of single PSS for the colonic stump and single PSS for the rectal stump. We defined the resected ring after cutting $0-0$ polypropylene as complete (intact of the resected ring), nearly complete (intact of the mucosa and defect of the muscularis propria), or incomplete (defects of mucosa and muscularis propria of the resected ring).

Results: A total of 47 patients were included in the study, 27 in the quadruple PSS group and 20 in the double PSS group. The resected ring for the rectal stump was complete in $88.9 \%$ of the quadruple PSS cases and in $70.0 \%$ of the double PSS cases. The resected ring for the colonic stump was complete in $92.6 \%$ of the quadruple PSS cases and in $75.0 \%$ of the double PSS cases. The resected ring for both stump was complete in $85.2 \%$ of the quadruple PSS cases and in $50.0 \%$ of the double PSS cases $(\mathrm{p}=0.012)$. Intraoperative adverse events were comparable between the two groups. The rate of complications in quadruple PSS cases and in double PSS cases were $22.2 \%$ and $30.0 \%$, respectively. The rate of anastomotic leak in quadruple PSS cases and in double PSS cases were $3.7 \%$ and $10.0 \%$, respectively. Other clinical outcomes were comparable between the two groups. The mesorectum specimen was complete in all patients except one patient of nearly complete.

Conclusions: Quadruple PSS for SST following TaTME for rectal cancer is a useful technique for completeness of resected rings to minimize the anastomotic-related morbidity.

\section{S178}

Defining The Burden of Diverticular Fistula Through Inpatient Admissions: A National Population Study In The United States

Joshua M Underhill, MD, MPH; Miles W Grunvald, MD; Ethan M Ritz, MS; Adan Z Becerra, PhD; Anuradha R Bhama, MD; Henry R Govekar, MD; Theodore J Saclarides, MD; Dana M Hayden, MD, MPH; Rush University Medical Center.

Introduction: Diverticular fistula, a pathologic connection from the colon to the skin or another organ, is an uncommon sequela of diverticular disease. It is generally considered an indication for surgery. The current literature is extremely limited in terms of defining the epidemiology of this disease process. This analysis aims to define the prevalence and demographics of fistulous diverticular disease on a national population level.

Methods: A retrospective review of the 2017 National Inpatient Sample (NIS) was conducted, using ICD-10-CM and ICD-10-PCS codes for diverticular disease, diverticularassociated fistulas, and associated surgeries in order to identify patients with diverticular disease both with and without associated fistulas. All available demographic factors were compared between groups, and several sub-group analyses were performed.

Results: A total of 7,159,694 discharges were recorded in the 2017 NIS database. 114,508 $(1.60 \%)$ patients had diverticular disease without fistula, while 3,515 (0.05\%) had diverticular-associated fistulas. Patients with diverticular fistula were more likely to be younger $(64.7$ v 67.9 years, $\mathrm{p}<0.0001)$ and female $(57.3 \%$ v $55.1 \%, \mathrm{p}=0.011)$ than patients with non-fistulizing disease. They were also more likely to undergo surgery ( $65.4 \% \mathrm{v} 26.1 \%, \mathrm{p}$ $<0.0001)$, to be admitted electively $(46.0 \% \mathrm{v} 12.7 \%, \mathrm{p}<0.0001)$, and to have a longer length of stay (LOS) (mean 8.42 v 5.22 days, $\mathrm{p}<0.0001)$. 2,299 of 3,515 patients $(65.4 \%)$ with diverticular fistula underwent surgery during their admission. Patients who underwent surgery were more likely to be male $(44.6 \%$ v $39.0 \%, p=0.001)$, to be admitted electively $(65.8 \%$ v $8.5 \%, \mathrm{p}<0.0001)$, and to have longer LOS (mean 9.10 v 7.13 day, $\mathrm{p}<0.0001$ ) relative to those that received medical treatment alone.

Conclusion: Diverticular fistula is a rare diagnosis for admission to the hospital, accounting for only $0.05 \%$ of total admissions and $2.98 \%$ of admissions for diverticular disease. However, this is more common than the previously reported rate of $<$ $0.1 \%$ of diverticular disease admissions. While surgery is generally indicated for patients with diverticular fistula, only $65.4 \%$ of patients underwent surgical treatment. Although this study is limited by its retrospective nature and use of administrative data, our findings help to elucidate the prevalence and patterns of inpatient admissions for diverticular fistula in the United States. 
S179

\section{CONSTRUCT VALIDITY AND RESPONSIVENESS OF THE DUKE ACTIVITY STATUS INDEX (DASI) AS A MEASURE OF RECOVERY AFTER COLORECTAL SURGERY}

Makena Pook, BHSc ${ }^{1}$; Hiba Elhaj, $\mathrm{MSc}^{2}$; Charbel El Kefraoui, $\mathrm{MSc}^{2}$; Saba Balvardi, $\mathrm{MD}^{3}$; Nicolo Pecorelli, MD, $\mathrm{MSc}^{2}$; L Lee, MD, $\mathrm{PhD}^{2}$; Liane S Feldman, $\mathrm{MD}^{2}$; Julio F Fiore Jr, PT, MSc, $\mathrm{PhD}^{2} ;{ }^{1}$ McGill University, Montreal, Quebec, Canada; ${ }^{2}$ Steinberg-Bernstein Centre for Minimally Invasive Surgery and Innovation, McGill University Health Centre, Montreal, Quebec, Canada; ${ }^{3}$ Department of Surgery, McGill University, Montreal, Quebec, Canada.

INTRODUCTION: Recent literature supports that preoperative self-reported functional capacity, as measured using the Duke Activity Status Index (DASI, a 12-item questionnaire), is a strong predictor of postoperative outcomes after major surgery. While the DASI may be a valuable tool to assess postoperative functional capacity, its measurement properties in the context of surgical recovery are yet to be determined. This study aimed to assess construct validity and responsiveness of the DASI as a measure of recovery after colorectal surgery.

METHODS AND PROCEDURES: We analyzed data from a clinical trial on early mobilization after colorectal surgery. Patients completed the DASI questionnaire preoperatively and at postoperative weeks 2 and 4 . Construct validity was assessed by testing the primary hypothesis that DASI scores at 2 and 4 weeks after surgery are higher in patients without postoperative complications vs with complications. Exploratory analyses were conducted on the association between DASI scores and (1) preoperative physical status, i.e. higher $(\mathrm{ASA} \leq 2)$ vs lower (ASA $>2)$, (2) postoperative stoma, i.e. without vs with stoma, (3) age, i.e. younger ( $\leq 75$ years) vs older ( $>75$ years), (4) time to readiness for discharge (TRD), i.e. shorter ( $<4$ days) vs longer ( $>4$ days), and (5) surgical approach, i.e. laparoscopic vs open. Responsiveness was assessed by testing the hypotheses that DASI scores are higher (1) preoperatively vs 2 weeks after surgery, (2) at 4 weeks vs 2 weeks after surgery. All hypotheses were informed by previous literature, defined a priori, and tested according to the Consensus-based Standards for the Selection of Health Measurement Instruments (COSMIN) criteria on measurement properties. Mean differences in DASI scores were obtained using linear regression.

RESULTS: One hundred consecutive patients undergoing colorectal surgery were included (mean age $65 ; 57 \%$ male; $81 \%$ laparoscopic; $37 \%$ rectal surgery). Mean DASI scores were $47.9 \pm 12.1$ preoperatively, $22.4 \pm 12.7$ at two weeks, and $33.2 \pm$ 15.7 at four weeks. The data supported our primary construct validity hypothesis and $3 / 5$ exploratory hypotheses. The two hypotheses tested for responsiveness were supported (Table).

CONCLUSIONS: The DASI discriminated between groups expected to have different levels of postoperative functional capacity and was responsive to the expected trajectory of recovery up to 4 weeks after colorectal surgery. Our findings support that the DASI questionnaire can be a simple and effective tool to assess recovery of functional capacity after colorectal surgery for research and clinical practice.

\begin{tabular}{|c|c|c|}
\hline \multirow{2}{*}{ Hypotheses tested for construct validity } & \multicolumn{2}{|c|}{ Mean Difference (95\% Cl) } \\
\hline & 2 Weeks & 4 Weeks \\
\hline \multicolumn{3}{|l|}{ Primary hypothesis } \\
\hline & $7.3(1.2$ to 13.4$)$ \\
\hline 1. Higher $(A S A \leq 2)$ vs lower physical status $(A S A>2)$ & $0.7(-6.5$ to 7.9$)$ & $-4.8(-14.6$ to 5.1$)$ \\
\hline 2. No postoperative stoma vs postoperative stoma & $6.6(1.2$ to 11.9$)$ & $10.0(3.2$ to 16.9$)$ \\
\hline 3. Younger ( $\leq 75$ years) vs older ( $>75$ years) & $3.2(-3.7$ to 10.1$)$ & $3.4(-5.5$ to 12.4$)$ \\
\hline 4. Shorter TRD ( $\leq 4$ days) vs longer TRD ( $>4$ days) & $6.6(0.3$ to 12.9$)$ & $8.2(0.4$ to 16.1$)$ \\
\hline 5. Laparoscopic vs open surgery & $7.2(1.0$ to 13.4$)$ & $3.6(-4.4$ to 11.7$)$ \\
\hline Hypotheses tested for responsiveness & \multicolumn{2}{|c|}{ Mean Difference $(95 \% \mathrm{Cl})$} \\
\hline 1. Baseline vs POW2 & \multirow{2}{*}{\multicolumn{2}{|c|}{$\begin{array}{c}25.1(21.6 \text { to } 28.6) \\
9.1(4.8 \text { to } 13.3)\end{array}$}} \\
\hline 2. POW4 vs POW2 & & \\
\hline
\end{tabular}

voldedity analysis warences above the minimal important difference of 6 points $(1 / 2 \mathrm{SD})$ considered meaningful. Construct
S180

\section{EMERGENCY COLECTOMIES IN THE NOAC ERA: A NATION-WIDE ANALYSIS DEMONSTRATING INCREASED COMPLICATIONS AND NEED FOR TRANSFUSIONS}

Jeongyoon Moon; Maryam Al Farsi; Mohammed AlQahtani; Daniel Marinescu; Carol-Ann Vasilevsky; Nancy Morin; Allison J. Pang; Marylise Boutros; Jewish General Hospital.

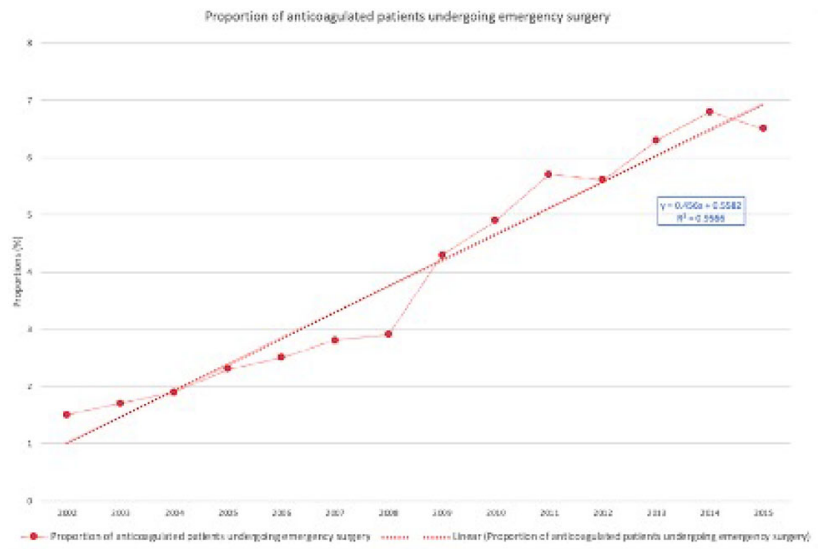

Introduction: Novel Oral Anticoagulants (NOAC) have been increasingly used since their introduction in 2010. The lack of available reversal agents poses a challenge for surgeons. The aim of this study was to evaluate the outcomes of emergency colorectal operations performed in patients on NOACs.

Methods: All adult patients on chronic anticoagulation who underwent emergency colorectal operations were identified from the National Inpatient Sample (NIS) database from 2002-2015. Chronic anticoagulation was defined using ICD-9/10 codes (V58.61/Z79.0). Two cohorts were compared: anticoagulated patients in the non-NOAC era (before 2010) and anticoagulated patients in the NOAC era (2010-2015). Patients only on aspirin, those who underwent elective procedures, and patients treated conservatively were excluded from the analysis. Outcomes of interest were postoperative surgical complications (defined as ileus, bowel obstruction, anastomotic leak, wound complications and surgical infections), mortality and need for transfusion. Multivariable regression was used to identify predictors of these outcomes.

Results: Of 35,726 chronically anti-coagulated patients,13,354 patients were in the non-NOAC group and 22,372 in the NOAC-group. Over the study period, there was a significant increase in the proportion of chronically anticoagulated patients undergoing emergency colorectal procedures $(\mathrm{R}=0.96)$ (Fig. 1). There was no difference in the mean age between the two groups ( $73 \pm 13$ years vs $73 \pm$ 12.4 years, $\mathrm{p}=0.505)$, but there were more males $(52 \%$ vs $50 \%, \mathrm{p}<0.001)$ in the NOAC group. Patients in the NOAC group were also medically more comorbid with higher percentages of patients with: congestive heart failure $(28 \%$ vs $23 \%$, p < $0.001)$, chronic lung disease $(27 \%$ vs $22 \%, \mathrm{p}<0.001)$, diabetes $(34 \%$ vs $26 \%$, p < $0.001)$, hypertension ( $74 \%$ vs $60 \%, \mathrm{p}<0.001)$, and renal failure $(24 \%$ vs $12 \%, \mathrm{p}<$ 0.001). The most common indication for anticoagulation in both groups was atrial fibrillation, followed by valvular heart disease, thrombophilia, and thromboembolic disease. On crude comparison, the rate of postoperative surgical complications and transfusions was higher in the NOAC-group (32\% vs $21 \%$, p $<0.001$ and $49 \%$ vs. $44 \%, \mathrm{p}<0.001$, respectively), but there was no significant difference in mortality between the two groups $(2.3 \%$ vs $2.2 \%, \mathrm{p}=0.368)$. On multivariable regression, after accounting for clinically significant covariates, anticoagulation in the NOAC era remained an independent predictor of postoperative surgical complications (OR $1.6195 \%$ CI 1.52-1.71) and transfusions (OR $1.0895 \%$ CI 1.04-1.14).

Conclusion: Anticoagulation is an important factor to consider in patients undergoing emergency colorectal surgeries. Specifically, anticoagulation in the NOAC era is associated with higher risk of postoperative surgical complications and need for transfusion. 


\section{S181}

\section{COST ANALYSIS OF INDOCYANINE GREEN FLUORESCENCE ANGIOGRAPHY FOR PREVENTION OF ANASTOMOTIC LEAKAGE IN COLORECTAL SURGERY}

Rachel Q Liu, MD, MSc ${ }^{1}$; Ahmad Elnahas, MD, MSc ${ }^{1}$; Nawar A Alkhamesi, MD, $\overline{\mathrm{PhD}}^{1}$; Jeffrey Hawel, MD, $\mathrm{MSc}^{1}$; Ephraim Tang, MD, MSc ${ }^{1}$; Abdulaziz Alnumay ${ }^{2}$; Christopher M Schlachta, MD, $\mathrm{CM}^{2} ;{ }^{1}$ Department of Surgery, Schulich Medicine and Dentistry, Western University, London, ON, Canada; ${ }^{2}$ CSTAR (Canadian Surgical Technologies \& Advanced Robotics), London Health Sciences Centre, London, ON, Canada.

Introduction: Indocyanine green, near infrared, fluorescence angiography (ICGFA) has been increasingly employed in colorectal surgery to assess tissue perfusion intraoperatively, with the goal of reducing the rate of anastomotic leakage. However, the economic impact of this intervention has not been investigated. The purpose of this study was to perform a cost analysis of the routine usage of ICG-FA in colorectal surgery from the hospital payer perspective.

Methods: A decision analysis model was developed for patients undergoing colorectal resection considering two scenarios: a) resection without the use of ICG-FA, b) resection with intra-operative ICG-FA for assessment of anastomotic perfusion (Fig. 1). Incorporated in the model were the current costs of ICG agent, fluorescence angiography equipment, surgery, and the probability of anastomotic leak with and without the use of ICG-FA. Estimates of anastomotic leak rate and effectiveness of ICG-FA were derived from a recent meta-analysis. Direct costs of surgical intervention and anastomotic leakage were obtained from a recent population-based administrative analysis. The base case was intentionally selected to represent the most conservative case costing estimates.

Results: Routine use of ICG-FA for colorectal surgery is cost-saving when cost analysis is performed using the following data: $8.6 \%$ (range $1.2 \%-21.2 \%$ ) risk of anastomotic leakage without ICG-FA, odds ratio of 0.46 (95\% CI: 0.34-0.62) for reduction of leak with ICG-FA, cost of uncomplicated laparoscopic colorectal resection surgery of $\$ 8,575.29$ (95\% CI: $\$ 6,263.88-11,126.12)$, cost of ICG-FA of $\$ 250$, and cost of anastomotic leakage not requiring re-operation of $\$ 9,934.50$ (95\% CI: $\$ 9,340.32-10,523.24)$

In one-way sensitivity analyses, routine use of ICG-FA was cost-saving as long as the cost of an anastomotic leak is more than \$5852.59, the cost of ICG-FA is less than $\$ 598.72$, the anastomotic leak rate (without ICG-FA) is higher than 5.3\%, and the odds ratio for reduction of leak with ICG-FA is less than 0.68

Conclusion: Routine use of ICG-FA in colorectal surgery was found to be a costsaving measure from a payor's perspective using the most conservative base case values derived from recent publications. However, the overall quality of available evidence is low. There is an urgent need for prospective, randomized controlled trials.

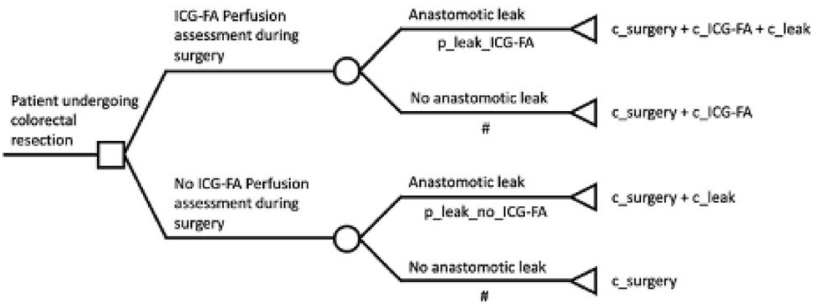

Figure 1 Decision tree depicting the possible perfusion assessment and outcome pathways for patients undergoing colorectal resection

\section{$\mathbf{S 1 8 3}$}

Colorectal Cancer in the 45 to 50 Age Group in the United States: A National Cancer Database (NCDB) Analysis

Thais Reif de Paula, MD ${ }^{1}$; Eric M Haas, MD ${ }^{1}$; Deborah S Keller, MD, $\overline{\mathrm{MS}^{2} ;{ }^{1} \text { University of Houston; }}{ }^{2} \mathrm{UC}$ Davis.

Introduction: Amidst increasing awareness of young-onset colorectal cancer (CRC), all guidelines in the United States (US) recently lowered the recommended CRC screening age from 45 to 50 in average-risk individuals. There is little data on the number of patients in this age group diagnosed with CRC prior to these changes. Our objective was to audit the historic US cases and trends of CRC in the 45-to-50year-old category prior to current screening recommendations.
Methods: All cases of colorectal adenocarcinoma in 45-to-50-year-old patients were queried from the NCDB from 2004-2017. Cases were stratified by colon, rectosigmoid junction (RSJ), and rectum. Patient and provider demographics and clinical data were analyzed. After standardization, a time series exploratory analysis was performed for the percent change/year by site with predictive forecasting. The main outcome measure was the trend in CRC by site over time and potential cases without screening guideline amendment in the 45 -to-50 cohort.

Results: We identified 56,130 CRC cases in the 45-to-50 demographic- $64.7 \%$ ( $\mathrm{n}=$ $36,293)$ colon, $25.1 \%(\mathrm{n}=14,076)$ rectal, and $10.3 \%(\mathrm{n}=5,761)$ rectosigmoid junction. Of these cases, $53.2 \%(n=39,410)$ were male and $85.9 \%(n=63,586)$ had no other comorbidities. At diagnosis, 32\% $(\mathrm{n}=17,946)$ had pathologic Stage III and $22.7 \%(\mathrm{n}=12,749)$ Stage IV disease. The absolute rate of all sites increased steadily over the time period. From the 5 and 10 -year forecast modeler analysis, there would be an estimated $11.4 \%$ increase in $2022(\mathrm{n}=3,249,95 \%$ CI $3,072-3,425)$ and $21.2 \%$ increase by 2027 ( $\mathrm{n}=3,535,95 \%$ CI $3,354-3,716)$ for colon cancer and an $11.6 \%$ increase in $2022(\mathrm{n}=1,315,95 \% \mathrm{CI} 1,196-1,434)$ and $28.6 \%$ increase by $2027 \mathrm{n}=$ $1,451,95 \%$ CI $1,328-1,574)$ in rectal cancer incidence.

Conclusion: Young-onset CRC has been steadily increasing in the US, specifically in the 45-to-50 age group. This demographic also had high rates of advanced stage disease at diagnosis. Without any intervention, the 5-year incidence rates for colon and rectal cancer would increase to an estimated $111.4 \%$ and $111.6 \%$, respectively, and the 10-year incidence rates would increase to $121.2 \%$ and $128.6 \%$, respectively. Thus, there is great potential benefit in lowering the recommended routine CRC screening age to 45 . Further study is needed to determine the actual impact these changes had on the US incidence and survivorship to both prevent and improve the quality of surveillance in CRC patients.
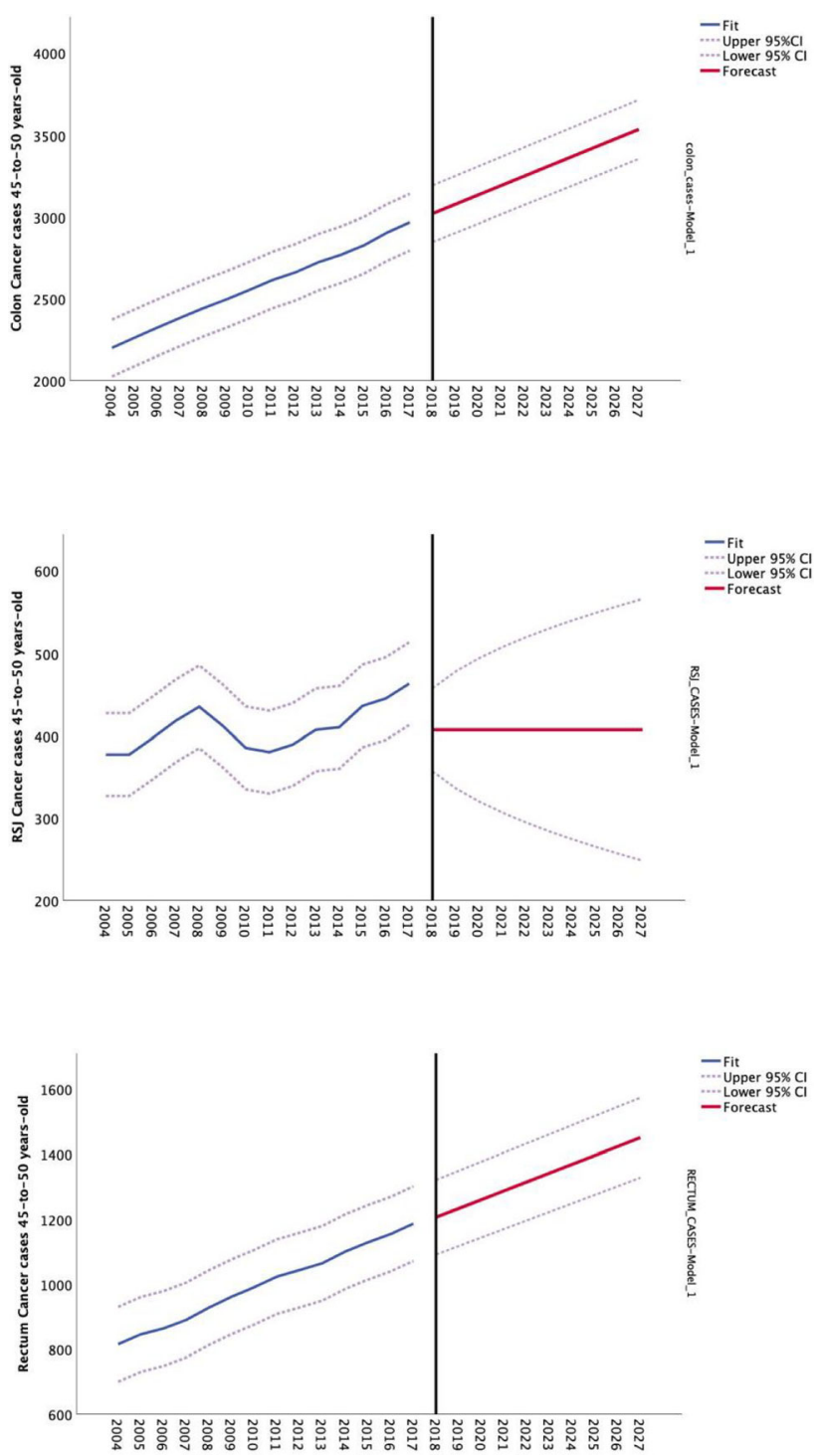


\section{S184}

Preoperative sarcopenia is associated with worse short-term outcomes following transanal total mesorectal excision (TaTME) for rectal cancer.

Jeremy E Springer, MD, MSc; Derek Chicarilli, MD; Danielle Coderre, BS; Catherine Beauharnais, MD, MS, MPH; Allison Crawford, MS; Jennifer A Baima, MD; Lacey J McIntosh; Jennifer S Davids, MD; Paul R Sturrock, MD; Justin A Maykel, MD; Karim Alavi, MD; University of Massachusetts.

Introduction: Malnutrition and deconditioning are known to significantly impact postoperative morbidity and mortality. Sarcopenia, measured by computed tomography (CT) body composition variables, is a marker of nutritional status and conditioning. The objective of this study is to evaluate the impact of sarcopenia on patient outcomes following transanal total mesorectal excision (TaTME) for rectal cancer.

Methods/Procedures: This was an institutional retrospective cohort analysis of consecutive patients who underwent TaTME for rectal cancer (Stage 1-4) from April 2014 to May 2020. Psoas muscle index (PMI) was calculated from diagnostic CT scans by measuring psoas muscle area at the third lumbar level using Terarecon Aquarius software@ (Fig. 1). Based on previous studies, patients in the lowest PMI tertile by gender were considered sarcopenic. Fisher's exact test and the MannWhitney U test were used to compare categorical and continuous variables respectively. Readmission rates and postoperative complications were compared between groups. Backwards selection was used to fit a logistic regression model determining the association of sarcopenia and any 30-day postoperative complication

Results: A total of 85 patients were analyzed; $63 \%$ were male, median age was 59 (IQR 51-65), and median BMI was 28 (IQR 24-32). Of the entire cohort, 34\% ( $\mathrm{n}=$ 29) were sarcopenic (median PMI 5.39 IQR 4.49-6.71). There was no significant difference in baseline characteristics between the sarcopenic and nonsarcopenic group when adjusting for comorbidities, ASA, preoperative and pathologic TNM stage and use of neoadjuvant chemoradiotherapy. Fifty-five percent of sarcopenic patients had a complication within 30 days compared to $24 \%$ of nonsarcopenic patients $(p=0.01)$. When comparing nonsarcopenic and sarcopenic groups, there was no significant difference in anastomotic leaks, surgical site infection or ileus ( $3.7 \%$ vs $0 \%, p=0.54 ; 3.7 \%$ vs $6.9 \%, p=0.61 ; 9.3 \%$ vs $24 \%, p=0.1$ respectively). Forty one percent of sarcopenic patients required hospital readmission within 30 days compared to $17 \%$ of their nonsarcopenic counterparts $(p=0.014)$. Sarcopenic patients also experienced significantly higher rates of post-operative small bowel obstruction $(10 \%$ vs $0 \%, p=0.04)$. Multivariable analyses revealed that sarcopenic patients have a fourfold increase in odds of experiencing a 30-day postoperative complication (OR 4.44, 95\% CI 1.6-12.4, $\mathrm{P}<0.05)$ after adjusting for gender.

Conclusion: In our retrospective study, preoperative sarcopenia was associated with increased 30-day postoperative complications following TaTME for rectal cancer. Postoperative complications may have serious oncologic implications by delaying adjuvant chemotherapy. Recognition of sarcopenia prior to undergoing TaTME for rectal cancer may provide an opportunity for early intervention with prehabilitation programs to reduce postoperative morbidity.

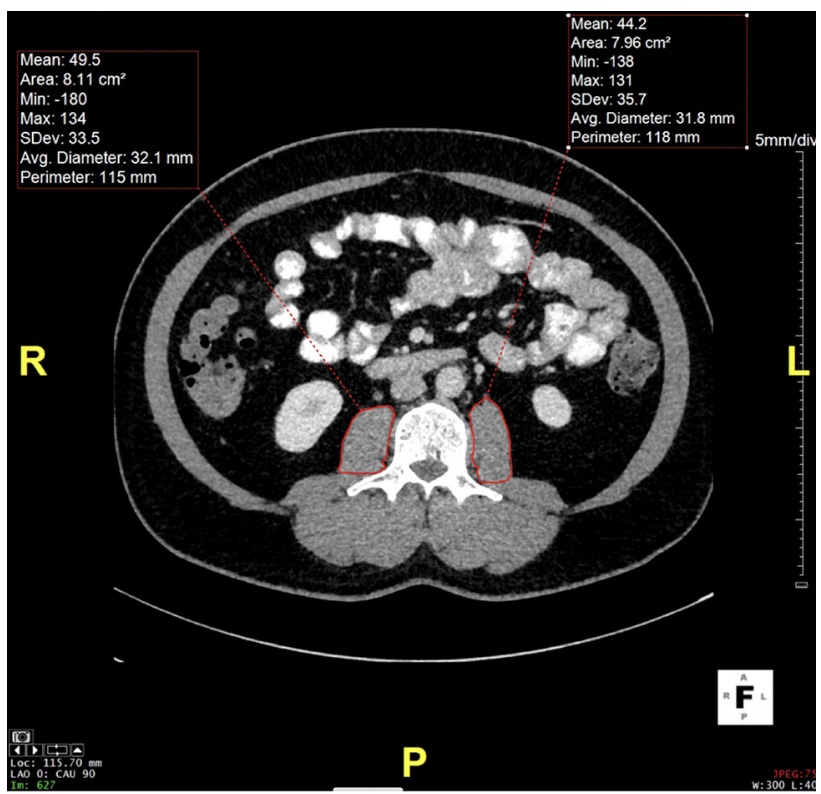

Representative psoas muscle index measurement. 


\section{S185}

\section{Morbidity following emergency and elective cholecystectomy: a retrospective comparative cohort study}

James Lucocq; Ganesh Radhakishnan; John Scollay; Pradeep Patil; NHS Tayside.

Introduction: Emergency laparoscopic cholecystectomies (LC) performed during an acute episode can be challenging due to acute inflammation but reduces the risk of further biliary pathology. The World Society of Emergency Surgery (WSES) and the updated Tokyo guidelines advise early cholecystectomy in emergency patients and for mild acute cholecystitis. A comprehensive comparison of post-operative outcomes between emergency and elective LCs has never been conducted and the relative morbidity associated with early cholecystectomies remains uncertain. Our aim was to compare post-operative outcomes between emergency and elective LCs to evaluate current practice.

Method: Emergency and elective LCs performed for biliary pathology across three surgical units between January 2015 and January 2020 were included in the study. The surgical units were located in a defined geographical region with a stable population of more than 416,090 people, based on mid-year 2017 population estimates, and less than a $1 \%$ migration rate. An emergency cholecystectomy was defined as an immediate, urgent $(<24 \mathrm{~h})$ or expedited $(<5$ days $)$ cholecystectomy with reference to the NCEPOD classification. Data was collected retrospectively from multiple regional databases using a deterministic records-linkage methodology. Complications, further imaging and interventions were recorded for post-operative stay and readmissions over a 100-day follow-up period and rates of outcomes were compared between emergency and elective groups using univariate analysis.

Results: Over the five-year period, 2775 cholecystectomies were performed (median age, 53 years; range, 13 - 92; Male:Female, 1:2.7). Emergency and elective cholecystectomies were performed in $20.5 \%$ and $79.5 \%$ of cases, respectively. The rate of post-operative complications in the emergency and elective cholecystectomy groups were $14.1 \%$ and $6.8 \%$, respectively ( $\mathrm{p}<0.00001$ ) (retained stones, $\mathrm{p}<$ 0.00001 ; collections, $\mathrm{p}=0.02$; bile leaks; $\mathrm{p}=0.0003$ ). Median post-operative stay in the emergency group was longer $(\mathrm{p}<0.00001)$ and the emergency group had a higher rate of prolonged stay ( $\geq 3$ days; $p<0.00001$ ). Fifteen percent of patients were re-admitted in the emergency group versus $6.1 \%$ in the elective group ( $p<$ $0.00001)$. Patients in the emergency group were more likely to require post-operative imaging and intervention $(\mathrm{p}<0.00001)$.

Conclusions: Despite current guidance, an emergency cholecystectomy remains a morbid procedure and we demonstrate an increased risk of emergency LC versus elective LC. The increased risk of an emergency LC needs to be weighed up against the risk of further attacks from biliary pathology until elective surgery. Our data indicates that we need to readjust our selection criteria for the 'emergency cholecystectomy patient' and identify patients who will specifically benefit from earlier surgery.

\section{S186}

\section{Do Normal Gallbladder Ultrasounds Rule out Acute Cholecystitis?}

Ahmed I Abdalla; Alikhan Karimi; Clarissa Ramirez; M H Almekdash, MA, MS PhD; Hassan Ahmed, MD, MRCSI; M H Nazim, MD; Texas Tech University HSC SOM.

Introduction: This study is designed to assess the reliability of ultrasound in identifying acute cholecystitis (AC) accurately based on the results of the histological specimen, which is considered the gold standard of diagnosis.

Methods: A retrospective chart review was conducted on 1000 patients, between the ages of 10 and 93, who underwent cholecystectomy surgeries between July 2016 and December 2018. Patients that had a gallbladder ultrasound (U/S) within 10 days of surgery that showed the presence of gallstones or sludge were included. A binomia logistic regression model was used to predict the likelihood of participants having positive pathological result. U/S results and the variables used to predict U/S such as: Gallbladder wall thickening $>4 \mathrm{~mm}$, Pericholecystic Fluid, and Sonographic Murphy's sign were included in the model while controlling for all other variables (Age, Gender, Race, Ethnicity, BMI, WBC count....etc.).

Results: A total of 444 patients met the inclusion criteria of the study. $214 \mathrm{U} / \mathrm{S}$ were negative. Of those, $70(32.3 \%)$ reports were found to be missed AC on pathology. The predictiveness of 230 positive U/S reports was like a coin toss. However, four variables were statistically significant predictors: Age, WBC count on admission, wall thickening $>4 \mathrm{~mm}$, and pericholecystic fluid. An increase in these variables resulted in a $2 \%, 11 \%, 200 \%$, and $300 \%$ increase in the likelihood of positive pathologic result, respectively. The logistic regression model was statistically significant, $\chi^{2}(81.53), \mathrm{p}<0.05$

Conclusions: Ultrasound screening criteria for $\mathrm{AC}$ are not significant predictors of pathology and should not be relied upon solely for the diagnosis. Parameters such as Age and WBC count should be considered in conjunction with ultrasound to increase the predictive power of the diagnostic method. 


\section{S187}

\section{Intraoperative Complexity and Risk Factors Associated with Conversion to Open Surgery During Laparoscopic Cholecystectomy in Eight Hospitals in Mexico City}

Gabriel Rangel-Olvera, MD, $\mathrm{MSc}^{1}$; Bianca Alanis Rivera, $\mathrm{MD}^{2}$; Jose Trejo-Suarez ${ }^{3}$; Jorge Yolik Ramirez-Marin ${ }^{4}$ J Jose Nicolas Garcia-Martin del Campo ${ }^{5}$; Jose Luis Beristain-Hernandez; ${ }^{6}$ Ignacio Del Rio-Suarez ${ }^{7}$ Adolfo Cuendis-Velazquez ${ }^{7}{ }^{1}$ Hospital General "Dr. Manuel Gea Gonzalez"/ Hospital General Milpa Alta; ${ }^{2}$ Hospital de Especialidades Dr. Belisario Domínguez/ Hospital Central Sur de Alta Especialidad PEMEX; ${ }^{3}$ Hospital General Xoco; ${ }^{4}$ Hospital General La Villa; ${ }^{5}$ Hospital General Ticoman; ${ }^{6}$ Centro Medico Nacional La Raza; ${ }^{7}$ Hospital General "Dr. Manuel Gea Gonzalez".

Background: The laparoscopic cholecystectomy (LC) is one of the most performed elective (ELS) or emergency surgery (ES) around the world. Conversion to open surgery $(\mathrm{COS})$ is reported to to occur at a rate of $10-15 \%$; it has been associated to a higher mortality and morbidity like bile duct injury (BDI), bile leak and bleeding, requiring transfusion or reintervention. Studies have postulated some related factors that influence a higher conversion rate (male gender, age, and comorbidities); however few studies have evaluated the association of this factors and the transoperative complexity with the conversion rate. The aim of the study is to evaluate the preoperative risk factors and trasnoperative complexity using the Parkland grading scale (PGS) with the conversion of LC to open surgery.

Methods: A retrospective, cross-sectional study was perform evaluating the patients and the videos of the LC from 8 different teaching hospitals of Mexico City from December of 2018 to January of 2020. Demographic and surgical variables were recorded (PGS, Total Laparoscopic Time-TT, Time before the clipping of the cystic structures-TCS, time to conversion-TTC, Estimated Blood Loss-EBL, BDI). The evaluation of the PGS was done by 2 surgeon (one MI and one HPB surgeon); this scale was also categorized as Non Complex Laparoscopic Cholecystectomy-nCLC and a Complex Laparoscopic Cholecystectomy-CLC by a PGS $>$ or $=$ to 3 ,. Logistic regression for univariate and multivariate analysis, using backwards stepwise strategy for the creation of the model.

Results: 430 LC were analyzed; 358(78.61\%) were women, $261(60.7 \%)$ were ELS and $169(39.3 \%) \mathrm{ES}$, the mean age was $44.06(\mathrm{SD} \pm 13.16)$ years. $21(4.8 \%) \mathrm{LC}$ were COS; the mean age of this group was $55(\mathrm{SD} \pm 12.95), 3(0.7 \%)$ were nCLC and 18 (4.19\%) CLC, with a mean PGS of 3.76(SD \pm 1.09 ), the mean TTC was 48.67 (SD $\pm 41.9)$, the EBL was $258(\mathrm{SD} \pm 260.22)$ and $6(1.4 \%)$ transoperative BDI were recognized on this group. The correlation coefficient between evaluators for the PGS was 0.892 . The univariate analysis showed a significant association with the conversion to open surgery with male gender, older age, age $>45$ years, presence of comorbidities, CLC and a higher EBL, the multivariate analysis produced a model using male gender, age, type of surgery, presence of comorbidities and a CLC with a 0.809 area under the ROC curve.

Conclusion: The recognition of the associated risk factors and a CLC can guide the surgeon to establish preoperative and bailout strategies during the procedure, recognizing a higher risk of COS and its higher morbidity.

\section{S189}

Post-operative recovery following laparoscopic cholecystectomy: a need to redefine the consent for cholecystectomy

James Lucocq; Ganesh Radhakishnan; John Scollay; Pradeep Patil; NHS Tayside.

Introduction: Laparoscopic cholecystectomy (LC) is the first-line treatment option for symptomatic gallstone disease and is one of the most frequently performed surgical procedures, with 409,612 being performed between 2009 and 2014 in the UK. Despite laparoscopic cholecystectomies being a common operation, the listed risks and incidences on consent forms vary greatly and generally lack adequate detail. Understanding the risks of LC is necessary before patients can make an informed decision regarding operative management. Our primary aim was to provide a comprehensive analysis of the post-operative course of these patients with detailed descriptions of post-operative complications, imaging, intervention, prolonged admissions and readmissions.

Method: Emergency and elective LCs performed for all biliary pathology across three surgical units between January 2015 and January 2020 were included in the study. We retrospectively collected data and followed each patient up for 100 days post-operatively using data-linkage methodology. Data collected included demographic data, operative data, post-operative recovery, imaging, additional interventions and re-admissions. Elective re-admissions under other medical specialties and emergency admissions unrelated to the cholecystectomy were excluded Results: A total of 2775 patients were identified (median age, 53 years (range 13-92); M:F, 1:2.7). There were no deaths in the cohort; however, $18.4 \%$ had a complicated postoperative period requiring either prolonged hospital stay, further imaging, additional interventions or re-admission. Two hundred and ninety-nine patients $(10.8 \%)$ had a prolonged post-operative stay and 289 patients $(10.4 \%)$ required post-operative imaging or intervention (e.g. CT, $4.6 \%$; MRCP, $4.6 \%$; USS, $2.9 \%$; ERCP, $1.7 \%$; Laparoscopy, $1.0 \%$ ). The rate of post-operative complications was $7.9 \%$ (220 patients; e.g. collections, $2.5 \%$; bile leaks, $2.0 \%$; retained stones, $1.6 \%)$. Two hundred and five patients $(7.4 \%)$ were re-admitted for assessment related to the LC (median admission length, 3 days; IQR, 4; median number of days following discharge, 9 days; IQR, 28) and $80.5 \%$ required imaging or intervention. Conclusion: Rates of complications, adverse post-operative recovery and re-admission are important indicators of morbidity that should inform patient and practitioner decision-making. Although having the reputation of largely an uncomplicated procedure, our data supports and illustrates the substantive morbidity associated with the operation. Referring physicians and patients should be counselled about the high morbidity rates so that they are well informed of their possible postoperative outcomes. This involves patient education and improved consent which should help decrease litigation. Our data should also prompt surgeons to take a more judicious approach when offering the procedure, particularly when the indication is insubstantial. 


\section{S191}

\section{Understanding Patient Preferences for Management of Symptomatic Gallbladder Disease}

Alex Lois; David Droullard, MD; Jenney Lee, MA; David Flum, MD, MPH; University of Washington.

Introduction: One in five people with gallstones ( $\sim 13$ million in the US) will develop symptoms in their lifetime yet only a fraction undergo cholecystectomy. The frequency of and factors associated with patients choosing medical management for gallbladder disease are not well understood. The goal of this study was to assess patient decision-making regarding cholecystectomy, experiences with medical therapies for gallbladder disease, and interest in a clinical trial comparing cholecystectomy with medical management for gallbladder disease.

Methods: We conducted a cross-sectional survey from January 2020 through May 2020 using Amazon's crowdsourcing platform, MTurk. Respondents completed a screening survey to determine gallbladder disease history and cholecystectomy status for a US $\$ 0.10$ stipend. Eligible participants without prior cholecystectomy were invited to complete the full survey, for an additional US $\$ 2.50$.

Results: There were 148 completed responses to the survey (93.7\% of attempted). Respondents were $54.1 \%$ female with a mean age of 36.2 (SD 11.3) years. $35.8 \%$ of respondents reported trying medications or supplements to manage their symptoms with $17.2 \%$ using lecithin and $21.6 \%$ using ursodeoxycholic acid (Ursodiol ${ }^{\circledR}$ ). $84.5 \%$ had attempted lifestyle modifications to manage their symptoms. Surgery was recommended for $59.5 \%$ of respondents of which $43.2 \%$ were planning for cholecystectomy. Among those recommended for operation, the most commonly cited reasons for pursuing surgery were symptom severity $(50.0 \%)$ and inability to care for family due to symptoms $(42.1 \%)$ while the most common reasons for not pursuing surgery were risk of surgery/anesthesia $(56.0 \%)$, trialing alternative therapies $(50.0 \%)$, and symptom severity $(38.0 \%)$. Factors involved in decision-making are detailed in Fig. 1. Interest in a clinical trial comparing medical alternatives to surgery was high with $67 \%$ of respondents willing to participate.

Discussion: A significant portion of patients with symptomatic gallbladder disease choose not to undergo cholecystectomy. One of the most common reasons for avoiding surgery was the use of alternative therapies, despite limited evidence to support their use. The lack of prospective comparison of cholecystectomy to medical therapies for the management of gallbladder disease, along with patient interest, support the need for a clinical trial.

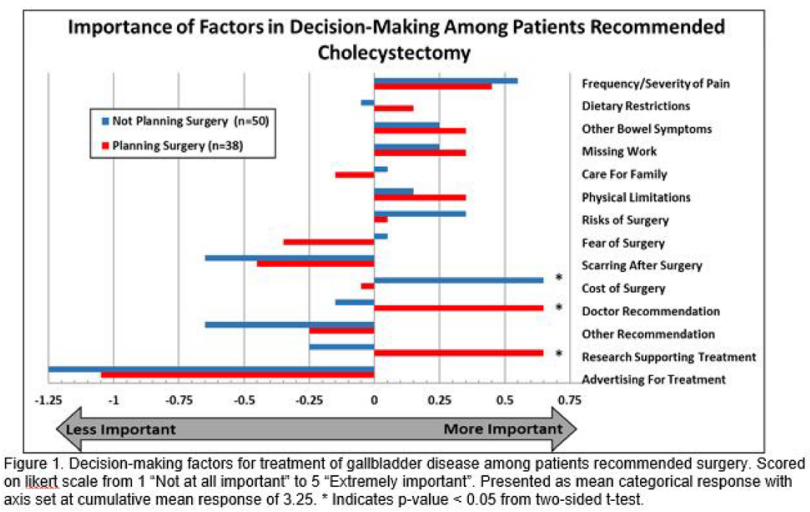

S192

Bile duct exploration with primary closure of duct without T-tube should be preferred in patients with failed endoscopic clearance of choledocholithiasis.

Dr. Vikram Singh Sodha, MBBS, MS, Mch, SGE trainee; Dr. Vivek Gupta, MBBS MS, ASSOCIATE PROFESSOR DEPT OF SGE; Prof. Abhijit Chandra, MBBS, MS, Mch, HOD and Professor DEPT OF SGE; KGMU Lucknow U.P. India.

Introduction: Preoperative endoscopic retrograde cholangiography (ERC) with bile duct clearance followed by laparoscopic cholecystectomy is the most common treatment for common bile duct (CBD) stones. However CBD stones that cannot be retrieved by ERC need surgical CBD exploration. Such stones are usually large, impacted and multiple with an inflammed $\mathrm{CBD}$ and often require open surgical exploration followed by primary CBD repair or T-tube insertion. The aim of study is to compare outcomes of primary CBD repair versus T-tube insertion in such cases. Patients and Methods: This was a combined retrospective $(2010-2019)$ and prospective (2019 to August 2020) observational study. Patients who underwent surgery for CBD stones after failed ERC clearance were included and divided into two groups, primary CBD repair or T-tube placement. The choice of procedure was as per surgeon's discretion. Patients clinical data, operative time, complications (Clavien-Dindo $3 \& 4)$, length of hospital stay and long term outcomes were compared.

Results: A total of 102 patients underwent surgical exploration of CBD. In primary repair groups $(\mathrm{n}=57), 40$ procedures were open, 10 laparoscopic converted to open and 7 laparoscopic. In T-tube groups $(n=45)$ there were 40 open and 5 laparoscopic procedures. In order to compare between homogenous groups, open procedures in primary CBD repair ( $n=40$, group A) were compared with open procedures with T-tube $(n=40$, group B). There was no difference in age, gender, ECOG status, stone size $(14.6 \pm 5.86 \mathrm{~mm}$ vs $14.3 \pm 6.70 \mathrm{~mm} \mathrm{p}=0.832)$ and number of stones $(2.05 \pm 1.15$ vs $2.47 \pm 1.04 \mathrm{p}=$ 0.09 ) between group $\mathrm{A}$ and $\mathrm{B}$. Operative time was significantly less in group A than group B $(2.5 \pm 0.50 \mathrm{~h}$ vs $3.15 \pm 0.38 \mathrm{~h} \mathrm{p}<0.01)$. Duration of hospital stay in group A was shorter than group $B(8.03 \pm 3.64$ days vs $12.15 \pm 5.50$ days, $\mathrm{p}<0.01)$. There was no difference in complications. T-tube group required two additional visits, one to get a tube cholangiogram and second to remove T-tube (mean of $15.15 \pm 2.86$ days after surgery). No evidence of stone recurrence or CBD stricture was observed in either group. Conclusions: Primary closure of CBD without T-tube is safe, preferred and translates into fewer hospital visits and lesser cost to the patient. 


\section{S194}

\section{Efficacy of endoscopic gallbladder drainage in patients with acute cholecystitis}

Anri Kaneta; Hirotaka Sasada; Tsuyoshi Sakai; Rin Itokawa; Shuichi Sato; department of surgery, Kensei Hospital.

Background: Early cholecystectomy is recommended for patients with acute cholecystitis. However, emergency surgery may not be indicated due to complications and severity. Patients requiring drainage are usually treated with percutaneous transhepatic gallbladder drainage (PTGBD) whereas patients with biliary duct stones undergo endoscopic removal of stones followed by endoscopic gallbladder drainage (EGBD). Herein, we investigated the efficacy of EGBD.

Methods: Overall, 101 patients who underwent laparoscopic cholecystectomy between September 2019 and September 2020 in our department were retrospectively analyzed.

Results: The patients $(n=101)$ were divided into three groups: control group that did not undergo drainage $(n=67)$, a group that underwent EGBD $(n=7)$, and a group that underwent PTGBD $(\mathrm{n}=27)$. Average operative time was 124.0, 191.9, and $150.7 \mathrm{~min}$, respectively. The control group had a significantly shorter operative time whereas there was no significant difference between the EGBD and PTGBD groups. The average amount of bleeding was $11.8 \mathrm{~g}, 7.1 \mathrm{~g}$, and $30.6 \mathrm{~g}$, respectively, and the control group had significantly less bleeding than the PTGBD group. We further divided patients into the following subgroups: patients requiring a 5-mm clip to ligate the cystic duct, patients requiring a 10-mm clip due to the thickness of the cystic duct, patients requiring an automatic suturing device, and patients undergoing subtotal cholecystectomy as cystic duct ligation was not possible. In the control group, the 5-mm clip was used more significantly and significantly fewer patients required an automatic suturing device and subtotal cholecystectomy. There was no significant difference between the EGBD and PTGBD groups in terms of the clip used or the need for an automatic suturing device and subtotal cholecystectomy. Conclusion: There was no significant difference between the EGBD and PTGBD groups in terms of operative time or bleeding amount when surgery was performed after gallbladder drainage for acute cholecystitis. The number of patients in whom cystic duct ligation with a 5-mm clip was not possible due to dilation was not significantly different between the EGBD and PTGBD groups. Therefore, EGBD showed similar clinical efficacy as PTGBD in addition to not requiring a drainage bag.

\section{S195}

\section{Predictors for Failure to Complete Laparoscopic Total Cholecystectomy}

Miya C Yoshida, DO; Takuya Ogami, MD; Eileen Bui, MD; Shahenda Khedr; Ken Hine, MD; Eduardo J Morales-Vicente, MD; Chun-Cheng Chen, MD, PhD; New York-Presbyterian/Queens.

Introduction: Laparoscopic total cholecystectomy (LTC) is considered the standard of care for gallbladder disease. It is now well known that in a difficult gallbladder LTC may be unsafe, resulting in a "bailout" surgery, which includes a subtotal excision or open conversion when unable to obtain the critical view of safety. We aim to characterize within a single-institutional experience the patient characteristics that predict need for bailout.

Methods: A retrospective chart review was conducted in our hospital from January 2016 to August 2019. We identified all patients who underwent an initially planned laparoscopic cholecystectomy during this time and separated patients into completed LTCs versus bailout procedures. Any planned open cholecystectomy or conversion as a consequence of confirmed bile duct injury was excluded from the analysis. Multivariable analysis was performed to identify independent factors predicting bailout cholecystectomy.

Results: We identified 2792 patients who underwent an initially planned laparoscopic cholecystectomy during this period: 2573 (92.2\%) were LTC and $219(7.8 \%)$ bailout. The latter group consisted of laparoscopic subtotal cholecystectomy ( $\mathrm{n}=$ $146,5.2 \%)$, conversion to open total cholecystectomy $(n=43,1.5 \%)$, and conversion to open subtotal cholecystectomy $(\mathrm{n}=30,1.1 \%)$. Complications were observed in 244 patients $(\mathrm{LTC}=175,6.8 \%$ vs. bailout $=69,31.5 \% ; \mathrm{p}<0.001)$. Specific complications included (all with $\mathrm{p}<0.01$ ): retained choledocholithiasis $(\mathrm{LTC}=45$, $1.8 \%$ vs. bailout $=10,4.6 \%)$; surgical site infection $(\mathrm{LTC}=22,0.9 \%$ vs. bailout $=$ $25,11.0 \%)$; and bile leak (LTC $=9,0.3 \%$ vs. bailout $=30,13.7 \%$ ). Median age was higher in the bailout cohort $(62$ vs $48, \mathrm{p}<0.001)$ and the bailout cohort had more comorbidities, including coronary artery disease $(13.2 \%$ vs $4.9 \%$ in LTC, p $<0.001)$ and diabetes $(28.8 \%$ vs. $14.2 \%, \mathrm{p}<0.001)$. The results of the multivariable analysis are listed in the table below.

Conclusion: Failure to achieve LTC occurred in $8 \%$ of planned cholecystectomies and the complication rate was higher compared to LTC. ASA classification $\geq 3$, male gender, history of smoking, and presence of adhesions were independently associated with conversion to any bailout procedure.

\begin{tabular}{lcc}
\hline \multicolumn{2}{l}{ Independent Factors Associated with Bailout Cholecystectomy } & \\
\hline & OR (95\% CI) & p-value \\
\hline ASA classification $\geq 3$ & $2.04(1.24-3.37)$ & 0.005 \\
Male gender & $1.83(1.10-3.03)$ & 0.020 \\
History of smoking & $2.08(1.20-3.59)$ & 0.009 \\
Intraoperative adhesions & $5.28(3.17-8.79)$ & $<0.001$
\end{tabular}


S196

Foregut Surgery is not associated with Chronic Opioid Use Postoperatively: An Analysis of the MarketScan Database

Ivy N Haskins, $\mathrm{MD}^{1}$; Emilie D Duchesneau, $\mathrm{BA}^{2}$; Tony Murante, $\mathrm{MD}^{1}$; Joyce Jhang, $\mathrm{MD}^{1}$; Paula D Strassle, $\mathrm{PhD}^{2}$; Timothy M Farrell, MD, FACS ${ }^{2} ;{ }^{1}$ University of Nebraska Medical Center; ${ }^{2}$ University of North Carolina.

Introduction: Postoperative opioid prescribing practices have decreased in recent years in an effort to combat the opioid epidemic in the United States. Currently, it is unknown if patients who undergo foregut general surgery operations are at risk for progressing to persistent postoperative opioid use. Therefore, the purpose of our study was to determine if patients who undergo foregut general surgery operations progress to persistent postoperative opioid use and to identify any patient- and surgery-specific factors associated with persistent postoperative opioid use following foregut general surgery operations.

Methods: Adult patients who underwent elective laparoscopic fundoplication, hiatal hernia repair, or Heller myotomy from 2010 to 2018 were identified within the IBM ${ }^{\circledR}$ MarketScan ${ }^{\circledR}$ Research Database. Patients with preoperative opioid use or those who underwent a concomitant procedure at the time of their foregut operation were removed from our analysis. Daily drug logs during the perioperative period, defined as between 14 days before and 14 days after surgery, and the postoperative period, defined as between 15 days and 365 after surgery, were derived based on National Drug Codes and days' supply information available from the outpatient prescription claims. Persistent postoperative opioid use was defined as at least 33\% of the proportion of days covered by opioid prescriptions during the following follow-up periods: 30, 90, 180, and 365 days. Patient demographic information (age, gender, region of residency) and clinical (procedure performed, indication for surgery, history of mental illness, medical comorbidities, and 30-day outcomes) risk factors for persistent postoperative opioid use at 365 days postoperatively were estimated using log-binomial regression.

Results: A total of 17,530 patients met inclusion criteria; 2,692 (15\%) underwent fundoplication, 13,438 (77\%) underwent hiatal hernia repair, and 1,400 (8\%) underwent Heller myotomy. 9,653 (55\%) patients had at least one prescription filled in the perioperative period (fundoplication: $60 \%$; hiatal hernia repair: $54 \%$; and Heller myotomy: 52\%). The number of patients with persistent postoperative opioid use declined from $978(6 \%)$ patients at 30 days postoperatively to $65(0.4 \%)$ at 365 days postoperatively. No patient or surgery risk factor was found to be significantly associated with persistent postoperative opioid use at 365 days postoperatively.

Conclusions: Only half of patients undergoing foregut general surgery operations require opioid prescriptions postoperatively. Furthermore, the risk of progression to chronic postoperative opioid use is less than $1 \%$. These findings further support currently guidelines that limit the number of opioid pills prescribed following general surgery operations.

\section{S198}

Self-Identified Black Patients Have No Difference in Access to Minimally Invasive Esophagectomy for Cancer Patients But Face High Incidence of Death with Open Approach

Syed F Haider, MD ${ }^{1}$; Weiyi Xia, MS ${ }^{2}$; Kasey L Wood, BS ${ }^{3}$; Patrick L Quinn, MD ${ }^{4}$; Ravi J Chokshi, MD, MPH, FACS ${ }^{5}$; ${ }^{1}$ Department of Surgery, Rutgers New Jersey Medical School; ${ }^{2}$ Rutgers School of Public Health, Rutgers University; ${ }^{3}$ University of Wisconsin School of Medicine and Public Health; ${ }^{4}$ The Ohio State University Wexner Medical Center; ${ }^{5}$ Department of Surgical Oncology, Rutgers New Jersey Medical School.

Background: Esophageal cancer is a common cancer globally. Advances in minimally invasive techniques have revolutionized surgical care. However, limited literature exists about the association between race and access to minimally invasive esophagectomy (MIE). This study investigated differences in access and clinical outcomes between open and MIE amongst black and white patients.

Methods: IRB-approved retrospective study utilizing data from the American College of Surgeons National Surgical Quality Improvement Program (ACS-NSQIP). Propensity score matching algorithm, Mantel-Haenszel tests and logistic regression were implemented.

Results: 6556 open and MIE cases were queried between 2010-2017. 96.75\% selfreported as white and $3.25 \%$ of patients self-reported as black. Multivariant analysis identified no statistically difference in access to MIE between black and white populations (OR [95\% Cl]: 0.59 [0.29, 1.21], p = 0.15). Similarly, no difference in access was observed after propensity matching (OR [95\% Cl]: $0.69[0.32,1.47] \mathrm{p}=$ 0.43 ). Additionally, no clinical outcomes in MIE were statistically significant due to limited sample size of black patients with MIE. However, black patients had a higher incidence of death (OR [95\% CI]: 1.94 [1, 3.610], $\mathrm{p}=0.053)$ with an open surgical resection.

Conclusion: Esophageal cancer carries a high morbidity and mortality. Both minimally invasive and open approach are used for patients that can undergo potentially curative. We demonstrated no statistically racial disparities in access to MIE. However, we observed significant risk for death with open surgical resection approach. This warrants further investigation into the barriers for black patients for MIE approach. 
S199

\section{Changes in Impedance Planimetry (EndoFLIP) Measurements at Follow-up after Peroral Endoscopic Myotomy (POEM)}

Mikhail Attaar, MD ${ }^{1}$; Harry J Wong, MD ${ }^{1}$; Hoover Wu, MD ${ }^{1}$; Michelle Campbell, $\mathrm{MD}^{1}$; Kristine Kuchta, $\mathrm{MS}^{2}$; Woody Denham, $\mathrm{MD}^{1}$; Steven Haggerty, MD ${ }^{1}$; John Linn, $\mathrm{MD}^{1}$; Michael B Ujiki, $\mathrm{MD}^{1} ;{ }^{1}$ NorthShore University HealthSystem; ${ }^{2}$ NorthShore University Research Institute.

Introduction: Numerous studies show changes in functional lumen imaging probe (FLIP) measurements after peroral endoscopic myotomy (POEM), but few report on FLIP measurements at follow-up esophagogastroduodenoscopy (EGD). The purpose of this study was to compare FLIP measurements after myotomy to those at followup EGD.

Methods and Procedures: Patients who underwent POEM with FLIP in the operating room and POEM patients who had EGD with FLIP at follow-up were included. FLIP measurements, including minimum diameter (Dmin), pressure, cross sectional area (CSA) and distensibility index (DI), were analyzed at a $30 \mathrm{~mL}$ balloon fill. Differences between measurements at different timepoints were assessed using a two-tailed Wilcoxon signed-rank test, with statistical significance set at $\mathrm{p}<0.05$. Results: A total of 80 patients who underwent POEM and 19 who underwent EGD with FLIP were analyzed. The average age was $64 \pm 16$ years and $49.1 \%$ of the patients were male. Mean preoperative Eckardt score was $6.8 \pm 5.5$, decreasing to $1.4 \pm 1.8$ at first follow-up and $1.2 \pm 1.6$ among patients who underwent EGD postoperatively. EGDs were performed at a median of 17 months after surgery. When compared to mean measurements obtained post-myotomy, at the time of EGD, pressure was found to be significantly lower $(\mathrm{p}=0.024)$ and DI significantly higher $(\mathrm{p}=0.049)$ (Table 1). Of the patients who underwent EGD, 64.3\% had an increase in DI, 59\% had evidence of reflux esophagitis and $79 \%$ met diagnostic criteria for reflux on esophageal $\mathrm{pH}$ monitoring. However, there was no correlation with development of esophagitis or reflux and whether patients had an increase or decrease in DI at follow-up.

Conclusions: There are significant changes in FLIP measurements between the time of surgery and at follow-up EGD. These results suggest that esophageal remodeling causes changes in LES geometry after POEM and post-operative FLIP targets immediately post-myotomy may need to be adjusted to account for these changes. Table 1

\begin{tabular}{llll}
\hline & Post-Myotomy & Follow-up & $\boldsymbol{p}$-value \\
\hline Total Patients & 80 & 19 & \\
Dmin (mm) & $11.1 \pm 1.8$ & $11.6 \pm 2.0$ & 0.751 \\
Pressure (mmHg) & $25 \pm 8$ & $22 \pm 6$ & $\mathbf{0 . 0 2 4}$ \\
Cross-sectional Area $\left(\mathrm{mm}^{2}\right)$ & $99 \pm 31$ & $110 \pm 39$ & 0.678 \\
Distensibility Index $\left(\mathrm{mm}^{2} / \mathrm{mmHg}\right)$ & $4.3 \pm 1.7$ & $5.0 \pm 1.7$ & $\mathbf{0 . 0 4 9}$ \\
\hline
\end{tabular}

\section{S200}

There is no correlation between a delayed gastric emptying of the gastric conduit and the occurrence of an anastomotic leakage after Ivor-Lewis esophagectomy

Benjamin Babic, $\mathrm{MD}^{1}$; Lars M Schiffmann, $\mathrm{MD}^{1}$; Christoph Mallmann, MD ${ }^{1}$; Dolores T Müller, $\mathrm{MD}^{1}$; Petra Schiller ${ }^{2}$; Seung-Hun Chon, MD ${ }^{1}$; Hans F Fuchs, $\mathrm{MD}^{1}$; Wolfgang Schröder, $\mathrm{MD}^{1}$; Christiane J Bruns, $\mathrm{MD}^{1}$; ${ }^{1}$ University of Cologne, Department for General, Visceral, Cancer and Transplant Surgery; ${ }^{2}$ Institute of Medical Statistics and Computational Biology (IMSB), University of Cologne, Germany.

Introduction: Thoracoabdominal esophagectomy with gastric pull-up is the gold standard for surgical therapy of esophageal cancer. Delayed gastric emptying of the gastric conduit (DGCE) is the most common functional disorder after Ivor-Lewis esophagectomy. Patients with DGCE significantly more often develop a pneumonia. It remains unclear, if there is a correlation between DGCE and the occurrence of an anastomotic leakage (AL). Aim of our retrospective analysis of more than 800 patients was to analyze if an anastomotic leakage is more likely to happen in patients with DGCE.

Methods: A total of 816 patients met inclusion criteria. All patients underwent an Ivor-Lewis esophagectomy due to esophageal or esophagogastric junction cancer between 2013 and 2018 at our center. Esophagogastric end-to-side anastomosis has been performed with a circular stapling device in all patients. The collective was divided into two groups, depending on the occurrence of an AL. AL occurred in 47 Patients (Group I) and was treated successfully in all patients by endoluminal therapy (ECCG grade II). Group I was compared to 769 patients (Group II) without $\mathrm{AL}$ regarding the occurrence of DGCE. In addition, pretherapeutic risk factors were compared among the two groups. DGCE was diagnosed if patients suffered from regurgitation on the 5 th postoperative day and if a dilatation of the gastric conduit was visible in chest $\mathrm{x}$-ray.

Results: $27.7 \%$ of all patients suffered from DGCE postoperatively. Female patients had a significantly higher chance to develop DGCE than male patients (34.4\%vs.26.2\% vs., $\mathrm{p}=0.040)$. Pneumonia was more common in patients with DGCE $(13.7 \%$ vs.8.5\%, $\mathrm{p}=0.025)$. Furthermore hospitalization was longer in these patients (median $17 \mathrm{~d}$ vs. $14 \mathrm{~d}, \mathrm{p}<0.001$ ). There was no difference in the rate of anastomotic leakage in patients with and without a DGCE and thus equal in both groups (5.8\%). Subgroup analysis showed that ASA $>3(7.6 \%$ vs. $4.4 \%, \mathrm{p}=0.05)$ and a squamous cell carcinoma $(9.8 \% \mathrm{vs} .4 .7 \%, \mathrm{p}=0.01)$ were independent risk factors for the occurrence of an anastomotic leakage.

Conclusion: Our study confirms that DGCE after Ivor-Lewis esophagectomy is a common finding in a standardized collective of patients in a high-volume center. This functional disorder is associated with a higher rate of pneumonia. Still, there is no correlation between DGCE and the occurrence of an anastomotic leakage after esophagectomy. The hypothesis, that an DGCE leads to a higher pressure on the anastomosis and leads to an $\mathrm{AL}$ in consequence, can be refuted. DGCE is no pathogenetic factor for an AL. 
S201

\section{A Dedicated Feeding Tube Clinic Reduces Emergency} Department Utilization for Feeding Tube Complications

Amber M Moyer, $\mathrm{MD}^{1}$; Teresa S Jones, $\mathrm{MD}^{2}$; Theresa L Morin, $\mathrm{MA}^{2}$; Krzystof J Wikiel, $\mathrm{MD}^{2}$; Carlton C Barnett, $\mathrm{MD}^{2}$; John T Moore, $\mathrm{MD}^{2}$; Thomas N Robinson, $\mathrm{MD}^{2}$; Edward L Jones, $\mathrm{MD}^{2} ;{ }^{1}$ University of Colorado School of Medicine, Department of Surgery; ${ }^{2}$ Denver VA Medical Center and University of Colorado School of Medicine, Department of Surgery.

INTRODUCTION: Feeding tubes (FT) can be placed endoscopically, surgically, or radiographically. They carry a significant risk of complication including infection, bleeding, leakage and unintentional removal. Routine follow-up is limited with inconsistent care guidelines and instructions from a variety of teams. To address this, we established a dedicated FT clinic to provide education, support and prophylactic replacement. We hypothesized that patients followed in FT clinic would reduce Emergency Department (ED) utilization.

METHODS: A retrospective review of patients who underwent FT placement at a Veterans Affairs hospital from 2010 to 2020. Baseline demographics, indications for placement, the number and reason for ED visits and the use of the FT clinic were recorded.

RESULTS: Ninety-three patients underwent FT placement during the study period. The majority were male $(89,96 \%)$ with an average age of $65(27-90)$. The most common indications were head and neck malignancy $(49,53 \%)$, malnutrition $(15$, $16 \%)$, neuromuscular disorders $(12,13 \%)$, and GI malignancy $(9,19 \%)$. The FT clinic began June 2016 and managed 18 patients, the majority of whom $(11,61 \%)$ had their FT placed prior to enrollment. Four patients (22\%) in the FT clinic group required ED visits for FT complications compared to $47(55 \%)$ in the standard-ofcare group $(\mathrm{p}=0.02)$. There was an average $0.8 \mathrm{ED}$ visits per patient (range $0-7)$ in the FT clinic group vs. 1.6 ED visits (range $0-20)$ in the standrad-of-care group $(\mathrm{p}=$ 0.28 ).

CONCLUSIONS: A dedicated FT clinic reduces ED visits by more than 50\%. No standardized care guidelines after FT placement exist, likely due to the involvement of multiple teams managing the patient and device, as well as the myriad of different tubes available. Biannual follow-up and prophylactic replacement decreases ED visits and should be considered at facilities that care for patients with feeding tubes.

\begin{tabular}{llll}
\hline ED Visits & & $\begin{array}{l}\text { Standard- } \\
\text { of-care n }=135\end{array}$ & $\begin{array}{l}\text { FT Clinic } \\
\mathrm{n}=15\end{array}$ \\
\hline Indications for ED Visit & $\begin{array}{l}\text { Tube Dislodgement } \\
\text { Leakage }\end{array}$ & $\begin{array}{l}47(35 \%) \\
19(15 \%)\end{array}$ & $6(40 \%)$ \\
& Clogged & $15 \%)$ \\
& Infection & $15(11 \%)$ & $4(27 \%)$ \\
& Tube Fracture & $14(10 \%)$ & $0(0 \%)$ \\
& Bleeding & $13(10 \%)$ & $0(0 \%)$ \\
& Pain & $7(5 \%)$ & $2(13 \%)$ \\
& Request Replacement & $0(0 \%)$ & $1(7 \%)$ \\
& Undocumented & $14(10 \%)$ & $0(0 \%)$ \\
FT Replaced & & $59(44 \%)$ & $6(40 \%)$ \\
\hline
\end{tabular}

\section{S202}

\section{POST-POEM REFLUX PATTERNS COMPARED TO GERD PATIENTS WITH NORMAL MOTILITY}

Brenda M Zosa, MD; Megan Murphy Belcaster, BS; Nathan Torell; Luke Funk, MD; Anne Lidor, MD; Amber Shada, MD; University of Wisconsin.

Introduction: Per-oral endoscopic myotomy (POEM) is gaining popularity as a treatment for achalasia, but has been associated with increases in gastroesophageal reflux (GERD) when compared to other treatment options. The incidence of GERD after POEM is $20-45 \%$, but most patients remain asymptomatic. Although the increased rate of GERD after POEM has been well established, the specific reflux pattern has not yet been characterized. This study serves to describe the specific acid reflux pattern after POEM as a treatment for achalasia, compared to patients with acid reflux and normal esophageal motility. We hypothesize that patients with GERD after POEM are more likely to have characteristics of stasis, such as greater reflux while supine and longer reflux episodes.

Methods: This was a retrospective analysis of prospectively collected data. Only patients with elevated DeMeester scores were included in this study. We compared reflux patterns between patients who were post-POEM and those with GERD, but normal motility. Variables including demographics, baseline Eckardt scores, and BRAVO tracings were gathered. Data between the two groups were compared using chi-square and students t-tests.

Results: A total of 40 patients were included in the study (17 post-POEM and 23 GERD patients). There were no significant differences in age, gender, or BMI between the two groups. Post-POEM and normal motility GERD patients had comparable DeMeester scores, but post-POEM patients had higher baseline Eckhardt scores. Post-POEM patients had fewer total reflux episodes and less percentage of post-prandial reflux, however had increased percentage of reflux while supine and mean duration of longest episode of reflux was double that of GERD patients. (Table 1).

Conclusion: GERD after POEM has a specific reflux pattern that is distinguishable from GERD in a patient with normal motility. Understanding what drives the reflux of post-myotomy patients is crucial to tailored treatment for patients experiencing GERD after treatment with POEM.

\begin{tabular}{|l|c|c|c|}
\hline Table 1 & Post-POEM n= 17 & GERD n=23 & $\boldsymbol{p}$ \\
\hline Age & $53 \pm 16$ & $51 \pm 14$ & 0.6 \\
\hline Male & $64.7 \%$ & $39.1 \%$ & 0.2 \\
\hline DeMeester Score & $42.7 \pm 28.2$ & $42 \pm 25.3$ & 0.9 \\
\hline Baseline Eckhardt Score & $6.9 \pm 2.7$ & $3.6 \pm 2.5$ & 0.001 \\
\hline Total Reflux Episodes & $59 \pm 53$ & $106 \pm 60$ & 0.02 \\
\hline \% Total time in Reflux & $11 \% \pm 6$ & $13 \% \pm 8$ & 0.43 \\
\hline \% Supine time in reflux & $19 \% \pm 13$ & $10 \% \pm 7$ & 0.01 \\
\hline \% Post-prandial time in reflux & $8 \% \pm 7$ & $14 \% \pm 8$ & 0.03 \\
\hline Longest episode (min) & $66 \pm 48$ & $33 \pm 14$ & 0.02 \\
\hline
\end{tabular}


$\mathbf{S 2 0 3}$

\section{Can Subjective Symptoms Predict Objective Findings in Gastroesophageal Reflux Patients?}

M Rasmussen, MD; M Ward, MD; L Hansen; K Chin; C Sanchez; G Ogola; S Leeds, MD; Baylor University Medical Center.

Introduction-Medical therapy is the first line treatment for gastroesophageal reflux disease, but surgical options are available and shown to be effective in those who fail medical management. There is no consensus for when a surgical evaluation is indicated. We set out to determine if the GERD HRQL questionnaire scores correlate to objective findings found in patients undergoing anti-reflux surgery as a way to predict when surgical consultation is warranted.

Methods-An institutional approved prospectively gathered database was used to look at all patients undergoing anti-reflux surgery from January 2014 to September 2020. Inclusion criteria included a diagnosis of gastroesophageal reflux disease by either ambulatory $\mathrm{pH}$ monitoring or identified LA grade $\mathrm{C} / \mathrm{D}$ esophagitis on endoscopy. Patients must have undergone a complete comprehensive esophageal workup that included taking the GERD-HRQL questionnaire, upper endoscopy, barium esophagram, esophageal manometry, and ambulatory $\mathrm{pH}$ monitoring. Analysis of the GERD-HRQL scores were compared to the objective endpoints to see correlation and predictability. Logistic regression analysis with restricted cubic splines was used to assess relationship between presence of objective findings and GERD HRQL questionnaire scores.

Results-There were 246 patients ( 165 female, 81 male) with a mean age of 54 . All patients had a comprehensive preoperative esophageal evaluation as well as a preoperative GERD HRQL questionnaire score. There was no significant correlation between GERD HRQL questionnaire score and their DeMeester score (correlation coefficient $=0.23$ ), or the presence or absence of a hiatal hernia regardless of size ( $\mathrm{p}$ $=0.89$ ). Patients with esophagitis had significantly higher average GERD HRQL questionnaire scores beyond those without esophagitis ( $40.1 \pm 18.9$ vs $30.4 \pm 19.1$, p $<0.0001)$. Patients with a score of 40 or greater had a $42 \%$ to $65 \%$ probability of having esophagitis as opposed to a score of 30 or less lowered the chances of having esophagitis to less than $35 \%$. An increased GERD-HRQL score was associated with a higher probability of having esophagitis (Fig. 1).

Conclusion-Usage of a GERD HRQL questionnaire score can potentially predict the chances of having erosive esophagitis on medical therapy which could help referring physicians identify those patients that are failing medical therapy and allow for prompt referral for surgical evaluation.

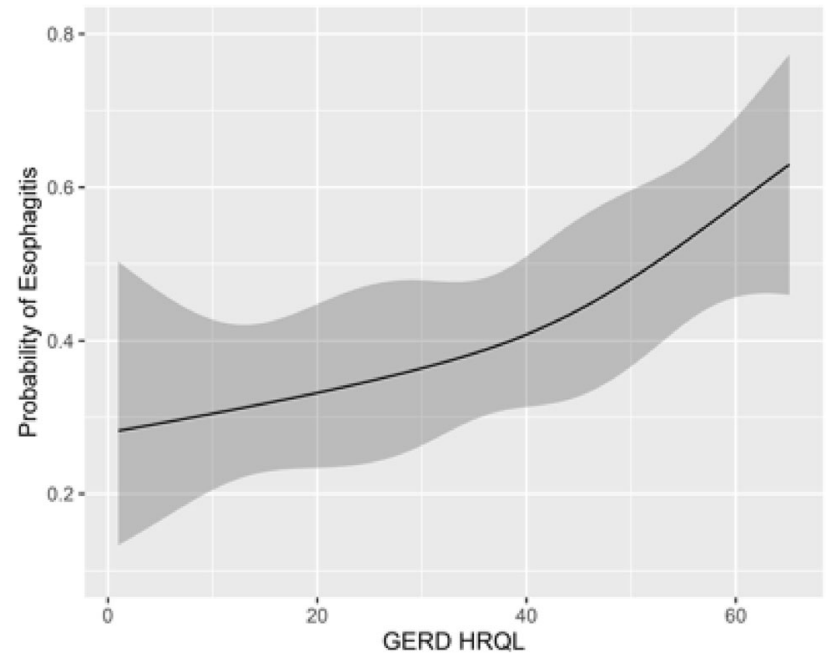

S204

\section{COMPARISON OF THE QUALITY OF LIFE BETWEEN LAPAROSCOPIC PROXIMAL GASTRETOMY WITH DOUBLE TRACT RECONTRUCTION WITH LAPAROSCOPIC TOTAL GASTRECTOMY FOR UPPER GASTRIC CANCER: A PROPENSITY SCORE MATCHING (PSM) ANALYSIS}

Mira Yoo, MD; So Hyun Kang, MD; Sang Jun Lee, MD; Yongjoon Won, MD Young Suk Park, MD; Sang-Hoon Ahn, MD; Yun-Suhk Suh, MD, PhD; Hyung-Ho $\mathrm{Kim}, \mathrm{MD}, \mathrm{PhD}$; Department of Surgery, Seoul National University Bundang Hospital, Seongnam, Korea.

INTRODUCTION: Laparoscopic proximal gastrectomy (LPG) with double tract reconstruction (DTR) is an emerging function-preserving surgery for upper one-third gastric cancer.

This is the first study that evaluates the quality of life of the patients who underwent LPG with DTR compared to laparoscopic total gastrectomy (LTG) by PSM.

METHODS AND PROCEDURES: We retrospectively collected 35 LPG with DTR and 35 LTG using PSM between January 2014 and December 2018 at Seoul National University Bundang Hospital. The patient's quality of life was analyzed using the European Organization for the Research and Treatment of Cancer (EORTC) Core Quality of Life (QoL) Questionnaire and the EORTC QoL Questionnaire-Stomach module from prospectively maintained database and hospital electronic medical chart.

Each QoL scale of both groups was compared at preoperatively, 3 months, 12 months, and 24 months after surgery. The surgical outcomes, postoperative complications, iron deficiency anemia, vitamin B12 replacement, and nutritional parameters were also analyzed. The gastric emptying scan was performed at postoperative 4 months, 12 months, and 24 months in the LPG with DTR group to evaluate the proportion of food passing through the remnant stomach versus the proximal jejunum.

RESULTS: There were no significant differences in the surgical outcomes and postoperative complications between the two groups. The LTG group had a higher level of C-reactive protein (CRP) postoperatively than the LPG with DTR group. (p $=0.017$ ).

The anemia and nutritional parameters were not different statistically between the two groups. However, vitamin B12 was more frequently replaced in the LTG group than the LPG with DTR group (68.6\% vs. $8.6 \%$, respectively) within the first 2 years after surgery. $(\mathrm{p}<0.001)$.

The LPG with DTR group showed higher physical function scores within the first year after surgery on QoL analysis. $(\mathrm{p}<0.001)$ The delta deviance in physical function and abdominal pain scales were more favorable in the LPG with DTR group than the LTG group within the first year after surgery. $(\mathrm{p}<0.001, \mathrm{p}=0.038$, respectively).

The gastric emptying scan showed that the food inflow to the remnant stomach increased over time $\left(r^{2}=0.958, p=0.014\right)$. It was correlated with improving postgastrectomy symptoms such as dysphagia, abdominal pain, reflux, and eating restriction.

CONCLUSION: The LPG with DTR showed better QoL with comparable surgical outcomes and complications over LTG for preventing vitamin B12 deficiency and improving life quality. 
S205

\section{REVIEW OF MEDICAL MALPRACTICE RELATED TO ANTI-REFLUX OPERATIONS: WHAT 20 + YEARS OF MEDICOLEGAL DATA HAS TAUGHT US?}

Asad J Choudhry, MD ${ }^{1}$; Bilal Malik, MD ${ }^{1}$; Jay Patel, MD ${ }^{1}$; Rashid Ahmed, MD ${ }^{1}$; Rami Abd-Rabu, MD ${ }^{2}$; Jason M Wallen, MD ${ }^{1}$; ${ }^{1}$ SUNY Upstate Medical University; ${ }^{2}$ Mayo Clinic, Rochester, MN.

Objective: Gastro-esophageal reflux disease (GERD) is common disorder with increasing prevalence in the US population. Anti-reflux surgery such as the Nissen fundoplication has an established role in the treatment of GERD. Through advancements in technique and approach, it has become a relatively safe procedure with a low complication rate; however medical errors, defined as adverse events do occur and affect patient care. There is very little data looking into reasons for litigation related to anti-reflux operations. Our aim was to understand the setting, contributing characteristics and outcomes of litigation targeting such procedure.

Methods: Westlaw Next (Thomson Reuters, New York, NY), an online medicolegal dataset with publicly available records from jurisdictions throughout the USA was queried. Using a Boolean surgery strategy with the terms medical malpractice and terms inclusive of anti-reflux operations we searched all medical malpractice cases. Only cases where the primary target for litigation was directly related to the anti-reflux operation were included. Remaining cases not directly targeting such procedures or repeat cases were excluded from our final analysis. Case information included: patient demographics, physician specialty, procedural characteristics, alleged reason for the malpractice claim and outcome(s) of trial.

Results: The search criteria yielded 226 case briefs; 62 cases met inclusion criteria. Dates of cases ranged from 1999 to 2019. Most of the patients involved in cases were female $24(58 \%)$; mean patient age was $49 \pm 20$. Failure to obtain adequate informed consent was noted in $8(13 \%)$ cases. The states of New York and California had the highest number of medical malpractice cases reported $10(16 \%)$. The most common alleged reason for litigation was related to a complication arising from the procedure. Overall $51 \%$ of cases were decided in favor of the defendant (physician). Median plaintiff award payout was $\$ 1,299,339$.

Conclusion: Although a majority of cases were decided in the favor of the defendant; settlements and cases with a jury verdict in favor of the plaintiff were shown to be costly. Surgical complications played a significant role in outcomes of malpractice litigation related to anti-reflux operations. Through understanding medical errors leading to physicians facing malpractice related to such procedures the future burden of litigation may be lessened if measures are enacted to mitigate such adverse events.

\section{S206}

Laparoscopic Revision Paraesophageal Hernia Repair; A 16-year Experience at a Single Institution.

Alex J Addo, MD, MPH ${ }^{1}$; Dylan Carmichael, $\mathrm{DO}^{1}$; Kelley Chan ${ }^{2}$; Andrew Broda ${ }^{3}$; Gabriel Mekel, MD ${ }^{1}$; Brian Dessify, $\mathrm{DO}^{1}$; Mustapha Daouadi, $\mathrm{MD}^{1}$; Jon Gabrielsen, $\mathrm{MD}^{1}$; Anthony Petrick, MD ${ }^{1}$; David M Parker, $\mathrm{MD}^{1}$; 1 GeisingVer Medical Center; ${ }^{2}$ Geisinger Commonwealth School of Medicine; ${ }^{3}$ University of Central Florida.

Background: Laparoscopic paraesophageal hernia repair (PEHr) is a safe and effective procedure for relieving symptoms associated with paraesophageal hernia $(\mathrm{PEH})$. Nonetheless, it is estimated that about $10-15 \%$ of patients will have symptomatic recurrence requiring additional surgical intervention. Revision surgery is technically demanding and may be associated with a higher rate of morbidity and poor patient reported outcomes. We present the largest study of perioperative and quality-of-life outcomes among patients who underwent laparoscopic revision PEHr.

Methods: A retrospective review of all patients who underwent laparoscopic revision paraesophageal hernia repair between February 2003 and October 2019, at a single institution was conducted. All revision of Type I hiatal hernias was excluded. The following validated surveys were used to evaluate quality of life outcomes: Reflux Symptom Index (RSI), Gastroesophageal Reflux Disease HealthRelated QOL (GERD-HRQL). Patient demographic, perioperative, and QOL data was?analyzed using univariate analysis.

Results: One hundred ninety patients were included in the final analysis $(63.2 \%$ female, $90.5 \%$ single revision, $9.5 \%$ multiple revisions) with a mean age, BMI and age adjusted Charleston score of $56.6 \pm 14.7$ years, $29.7 \pm 5.7 \mathrm{~kg} / \mathrm{m} 2$ and $2.04 \pm$ 1.9 respectively. The study cohort consisted of type II $(49.5 \%)$, III $(46.3 \%)$ and IV hiatal hernia $(4.2 \%)$ respectively. Most patients underwent either a complete $(68.7 \%)$ or partial $(27.7 \%)$ fundoplication. A Collis gastroplasty was performed in $14.7 \%$ of patients. The median follow-up was 17.6 months. The overall morbidity and mortality rate were $15.8 \%$ and $1.1 \%$ respectively. The 30 -day readmission rate was $9.5 \%$. Although there was no significant improvement in GERD-HRQL scores $(0.68 \%, \mathrm{p}>0.05)$ at latest follow-up, there was significant improvement in mean RSI score $(46.4 \%, \mathrm{p}<0.001)$ from baseline within the study population at latest follow-up. Furthermore, there was no significant difference in QOL between patients who had a single or multiple revision at latest review. The recurrence rate was $13.9 \%$ with $6.3 \%$ requiring a surgical revision.

Conclusion: Laparoscopic revision PEHr is safe and effective at a high-volume center. Revisions are associated with long-term improvement in QOL outcomes, despite the high rate of long-term antireflux medication use. The rate of recurrent paraesophageal hernia remains low with few patients requiring a second revision. 


\section{S207}

\section{Evaluation of Post-Operative Dysphagia Following Anti-Reflux Surgery}

Jacques A Greenberg, MD ${ }^{1}$; Dessislava I Stefanova, MD ${ }^{1}$; Fernando Valle Reyes, $\mathrm{MD}^{2}$; Jessica W Thiesmeyer, MD ${ }^{1}$; Caitlin Egan, MD ${ }^{1}$; Mengyuan Liu, MD Brendan M Finnerty, MD ${ }^{1}$; Thomas J Fahey III, MD ${ }^{1}$; Rasa Zarnegar, MD ${ }^{1}$; ${ }^{1}$ New York-Presbyterian Hospital / Weill Cornell Medical Center; ${ }^{2}$ Weill Cornell Medical Center.

Introduction: Endoscopic functional lumen imaging probe (EndoFLIP) technology is gaining traction in the performance of anti-reflux surgery (ARS). The factors affecting the development of post-operative dysphagia are poorly understood. Postprocedure distensibility index (DI) $<2 \mathrm{~mm}^{\wedge} 2 / \mathrm{mmHg}$ has been identified as a predictor of post-operative dysphagia. We aim to investigate the correlation of intraoperative esophagogastric junction (EGJ) characteristics and procedure type with post-operative dysphagia following ARS.

Methods: Robotic ARS was performed on 177 consecutive patients with pathologic reflux utilizing EndoFLIP technology. Intraoperative EGJ measurements including DI, cross-sectional area (CSA), and high-pressure zone (HPZ) length were collected. Dysphagia was assessed post-operatively using the Bazaz Score.

Results: The median pre-operative DI for all procedures was $2.6 \mathrm{~mm}^{\wedge} 2 / \mathrm{mmHg}$. There was no difference in post-operative DI between procedures (Hill: $1.0 \mathrm{~mm}^{\wedge} 2$ / mmHg, Nissen: $1.0 \mathrm{~mm}^{\wedge} 2 / \mathrm{mmHg}$, Toupet: $1.2 \mathrm{~mm}^{\wedge} 2 / \mathrm{mmHg}$, Linx: $1.0 \mathrm{~mm}^{\wedge} 2 /$ $\mathrm{mmHg}, \mathrm{p}=0.24)$. The median pre-operative HPZ length was $2 \mathrm{~cm}$. Post-operative HPZ length differed among procedures (Hill: $3 \mathrm{~cm}$, Nissen: $3.5 \mathrm{~cm}$, Toupet: $3 \mathrm{~cm}$, Linx: $2.5 \mathrm{~cm}, \mathrm{p}=0.02)$. The overall mean change in pre-operative DI compared to post-operative DI differed among procedures (Hill: $-2.76 \mathrm{~mm}^{\wedge} 2 / \mathrm{mmHg}$, Nissen: $1.1 \mathrm{~mm}^{\wedge} 2 / \mathrm{mmHg}$, Toupet: $-2.2 \mathrm{~mm}^{\wedge} 2 / \mathrm{mmHg}$, Linx: $-1.8 \mathrm{~mm}^{\wedge} 2 / \mathrm{mmHg}, \mathrm{p}<0.01$ ), but overall mean change in HPZ length did not (Hill: $1.5 \mathrm{~cm}$, Nissen: $1.3 \mathrm{~cm}$, Toupet: $1.5 \mathrm{~cm}$, Linx: $1.1 \mathrm{~cm}, \mathrm{p}=0.27)$. The change in HPZ length between post$\mathrm{HH}$ repair and post-procedure was significantly less for Linx than for the other procedures (Hill: $1.0 \mathrm{~cm}$, Nissen: $0.7 \mathrm{~cm}$, Toupet: $0.5 \mathrm{~cm}$, Linx: $0 \mathrm{~cm}, \mathrm{p}=0.02$ ). Seventy-nine patients had pre-operative dysphagia. Thirteen patients developed new or worsening post-operative dysphagia at 3-month follow-up (Hill: 1/17 [5.9\%], Nissen: $3 / 54$ [5.7\%], Toupet: 4/92 [4.4\%], Linx: $5 / 14$ [35.7\%], $\mathrm{p}<0.01)$. The median pre-operative and post-operative DI of patients that developed dysphagia was $2.0 \mathrm{~mm}^{\wedge} 2 / \mathrm{mmHg}$ and $1.2 \mathrm{~mm}^{\wedge} 2 / \mathrm{mmHg}$, and that of those who did not develop postoperative dysphagia was $2.5 \mathrm{~mm}^{\wedge} 2 / \mathrm{mmHg}$ and $0.9 \mathrm{~mm}^{\wedge} 2 / \mathrm{mmHg}(\mathrm{p}=0.464$ and 0.090). The mean overall change in DI and HPZ length were similar between those who developed post-operative dysphagia and those who did not (dDI $-1.1 \pm$ $1.2 \mathrm{~mm}^{\wedge} 2 / \mathrm{mmHg}$ vs $-1.8 \pm 1.7 \mathrm{~mm}^{\wedge} 2 / \mathrm{mmHg}, \mathrm{p}=0.29 . \mathrm{dHPZ} 1.2 \pm 1.0 \mathrm{~cm}$ v $1.4 \pm$ $0.8 \mathrm{~cm}, \mathrm{p}=0.22$ )

Conclusions: Post-operative DI was similar between procedures, and there was no correlation with dysphagia. Linx placement was associated with higher rates of postoperative dysphagia despite a shorter post-procedure HPZ length and similar postoperative DI when compared to other methods of LES augmentation. Further investigation into the etiology of dysphagia driven by Linx is necessary, as it appears to be independent of EGJ physiology.

\section{S208}

\section{Long-Term Outcomes Following Esophagectomy Between Older and Younger Adults with Esophageal Cancer}

Aaron R Dezube, MD; Lisa Cooper, MD; Emanuele Mazzola, PhD; Daniel P Dolan, MD; Suden Kucukak, MD; Luis E De-Leon, MD; Clark Dumontier, MD; Bayonle Ademola, BS; Emily Polhemus, BS; Raphael Bueno, MD; Abby White, DO; Scott J Swanson, MD; Michael T Jaklitsch, MD; Laura Frain, MD; Jon O Wee, MD; Brigham and Women's Hospital.

Objective: Recovery from esophagectomy in older adults is associated with increased risk of decline in functional status and weight loss. This may lead to differences in survival as a function of age, but the pattern of mortality has not been carefully studied.
Methods: Retrospective analysis of all patients with esophageal cancer undergoing esophagectomy from 2005-2020. Differences in outcomes were stratified according to 2 age groups: $<75$ and $\geq 75$ years old.

Results: 1135 patients were included: $979(86.3 \%)$ patients were $<75(86.3 \%)$, and $156(13.7 \%)$ were $>75$ years-old. Baseline characteristics were similar aside from higher median BMI, albumin level and active smoking history, as well as lower rates of CAD, HTN and median ECOG scores in the younger cohort (all $\mathrm{p}<0.001$ ).

Younger patients received more neoadjuvant chemoradiation $(83.4 \%$ vs. $70.5 \%$; $p<$ $0.001)$ and adjuvant therapy ( $14 \%$ vs. $4.6 \% ; \mathrm{p}=0.002$ ). Neoadjuvant chemoradiation utilization significantly increased for both age groups in 2 epochs: 2005-2014 and 2015-2020 (Range: $79.6 \%$ to $88.7 \%$ in patients $<75$ years old, $62.2 \%$ to $79.7 \%$ in those $\geq 75$ years old; both $\mathrm{p}<0.05$ ).

The only differences noted in tumor characteristics with age were lower rates of lymphovascular invasion $(18.3 \%$ vs. $25.6 \%$ respectively; $p=0.04)$, proximal $(0.92 \%$ vs. $3.9 \%)$ and mid esophageal tumors $(10.2 \%$ vs. $12.8 \% ; \mathrm{p}=0.007)$, and higher rates of signet cell $(14.5 \%$ vs. $8.3 \%$ respectively; $p=0.05)$ in the younger group. Clinical and pathologic stages were similar between groups (all p >0.05). Operative approaches were similar, but younger patients had higher rates of McKweon esophagectomy $(48.9 \%$ vs. $39.7 \%$; $=0.02)$.

Ninety-day survival was $97.2 \%$ vs. $92 \%$ for younger vs. older adults $(\mathrm{p}<0.05)$ 1-, 3- and 5- year survival (Fig. 1 ) were $84.3 \%$ vs. $71 \%, 62.5 \%$ vs. $37.4 \%$, and $50.7 \%$ vs. $32.3 \%$ respectively for younger vs. older adults (all $\mathrm{p}<0.05$ ), with a median survival of 62.2 vs. 21.5 months respectively $(p<0.05)$. KM curve deviates for the first 18 months, and then becomes parallel, suggesting differences in survival were due to factors in that time period.

Our multivariable Cox model showed older age ( $\geq 75$-year-old) was associated with reduced overall-survival (HR $2.0495 \%$ CI 1.5-2.8; p < 0.001) but not diseasefree survival (HR $1.195 \%$ CI $0.78-1.6$; $\mathrm{p}=0.54$ ).

Conclusions: Despite similar disease-free survival rates, long-term survival was decreased in the older age group as compared to younger patients, with a drop in mid- term survival. This may be related to unmeasured factors such as frailty, treatment toxicity and competing causes of death, for which further study is warranted.

Figure 1: Kaplan-Meier Survival overall survival curve by age group in patients with esophageal cancer undergoing esophagecton

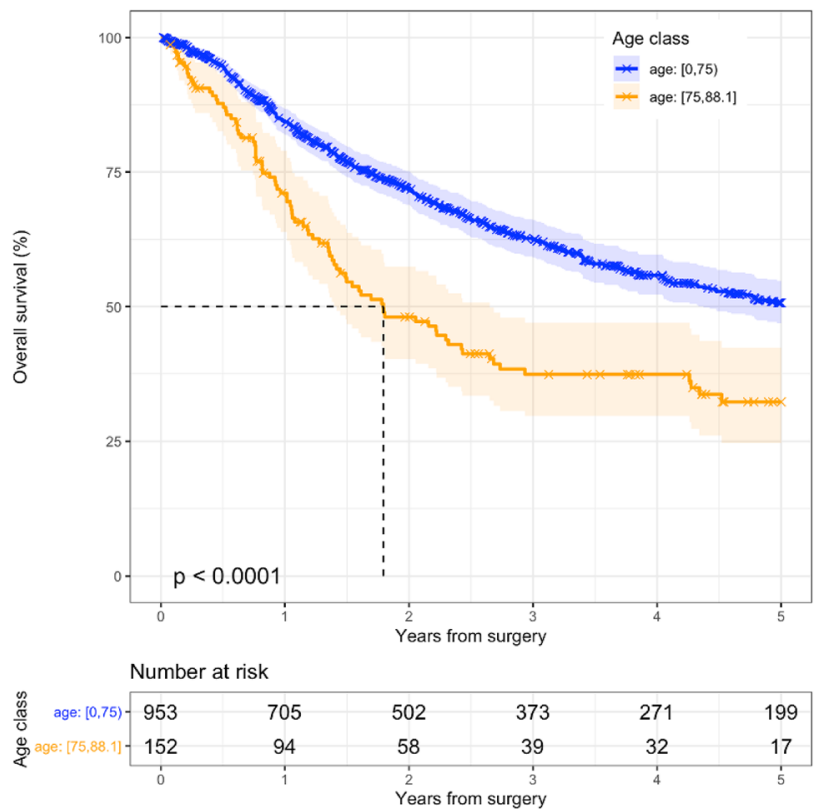


S209

\section{An Analysis of Predictive Factors and Clinical Outcomes of Pleural Tears During Laparoscopic Esophageal Surgery}

Matthew M Snyder, MD, MS ${ }^{1}$; Zachary M Tyerman, MD, MSc ${ }^{1}$; Paul J Devlin, $\mathrm{MD}^{1}$; Ezra N Teitelbaum, MD ${ }^{1}$; Thomas K Varghese, MD, $\mathrm{MS}^{2}$; Eric S Hungness, $\mathrm{MD}^{1}$; Nathaniel J Soper, $\mathrm{MD}^{3} ;{ }^{1} \mathrm{McG}$ aw Medical Center of Northwestern University; ${ }^{2}$ University of Utah Health Hospitals and Clinics; ${ }^{3}$ University of Arizona College of Medicine- Phoenix.

INTRODUCTION: Paraesophageal hernia (PEH) repair, Nissen fundoplication, and Heller myotomy are three common laparoscopic esophageal surgeries that involve dissection of the diaphragmatic crura and mobilization of the mediastinal esophagus. Tears in the mediastinal parietal pleura can occur during these maneuvers, resulting in capnothorax and potential hemodynamic and respiratory instability. This study analyzes predictive factors and clinical outcomes of pleural tears that occur during laparoscopic esophageal surgery for benign disease.

METHODS: A single-surgeon prospective database of laparoscopic esophageal operations was analyzed. The presence of pleural tear was recorded for each operation, as were any resultant hemodynamic or respiratory changes. Additional data, including primary operator, procedure duration, need for adhesiolysis, EBL, and length of stay were all recorded prospectively.

RESULTS: 382 laparoscopic operations were performed: 64 PEH repairs, 199 Nissen fundoplications, and 119 Heller myotomies. 57 cases were reoperations. Pleural tears occurred in 44 (12\%) cases, of which 13 (30\% of pleural tears, $3 \%$ of all cases) resulted in transient hemodynamic or respiratory instability. All 13 cases were resolved successfully by non-invasive maneuvers. Comparing cases with and without pleural tear, there were no differences in rates of other complications ( 5 vs. $12 \% ; \mathrm{p}>$ 0.05 ) or length of stay (mean 1.4 vs. 1.3 days; $p>0.05$ ). The incidence of pleural tears was significantly different for each procedure: PEH repair $(26 \%)$, Nissen $(11 \%)$ and Heller $(3 \%)$ ( $\mathrm{p}<0.05$ for each paired comparison). Primary and re-operations had a similar incidence of pleural tears $(12 \%$ vs. $11 \%$; $p>0.05)$. The level of training of the primary operator did not affect the pleural tear rate (resident: $8 \%$, fellow: $14 \%$, attending: $12 \%, \mathrm{p}>0.05$ ). When patient demographics were compared between cases with and without pleural tears there were no differences in gender distribution, age, or BMI ( $\mathrm{p}>0.05$ for all paired comparisons). In addition, cases with and without pleural tears did not differ in terms of length, EBL, or need for adhesiolysis ( $\mathrm{p}>0.05$ for all paired comparisons).

CONCLUSION: In this series of laparoscopic esophageal operations, pleural tears occurred in $12 \%$ of cases with transient hemodynamic or respiratory instability occurring in only $3 \%$ of all cases. All tears and their sequelae were managed intraoperatively without the need for tube thoracostomy. There were no demographic or operative factors associated with pleural tears, and their occurrence did not affect short term outcomes in any patient group. Our analysis concludes that, while there are no predictive factors, intraoperative pleural tears have no significant impact on post-operative outcome.

\section{S210}

\section{Intraoperative diagnosis and treatment of achalasia using EndoFLIP during Heller Myotomy and Dor fundoplication}

Yi Y Law, MD; Duc T Nguyen, MD, PhD; Leonora M Meisenbach, DNP, RN, ACNPBC; Ray Chihara, MD, PhD; Edward Y Chan, MD, FACS; Edward Graviss, PhD, MPH, FIDSA; Min P Kim, MD, FACS; Houston Methodist Hospital.

Introduction: Manometry is the gold standard diagnostic test for achalasia. However, there are incidences where manometry cannot be obtained preoperatively or the results of manometry is inconsistent with patient's symptomatology. We aim to determine if intraoperative use of EndoFLIP can provide diagnosis of achalasia and provide objective information during Heller myotomy and Dor fundoplication. Methods: To determine the intraoperative diagnostic EndoFLIP values for patients with achalasia, we obtained initial EndoFLIP values on our cohort of patients with definitive diagnosis of achalasia and another cohort with definitive diagnosis of hiatal hernia. We determined the optimal cut off points of the distensibility index (DI) using the receiver operating characteristic analysis with Youden index. We then analyzed patients without definitive diagnosis of achalasia or diagnosed with achalasia on manometry with symptoms inconsistent for achalasia. To evaluate usefulness of EndoFLIP values during Heller myotomy and Dor fundoplication, we obtained a cohort of patients with EndoFLIP values obtained after Heller myotomy and after Dor fundoplication as well as Eckardt score before and after surgery.

Results: Our analysis of 169 patients (133 hiatal hernia and 36 achalasia) showed that patients with DI $<0.8$ have a $>99 \%$ probability of having achalasia, while D $>2.3$ have a $>99 \%$ probability of having hiatal hernia. Patients with a DI $0.8-1.3$ have a $95 \%$ probability of having achalasia, and patients with a DI $1.4-2.2$ have a $94 \%$ probability that the patients of having hiatal hernia. There were six patients without definitive or questionable diagnosis, two of the patients had DI $<$ 0.8 and had surgical treatment for achalasia and four of the patients had DI $>2.3$ and had surgical treatment for hiatal hernia. All of them had good clinical outcome. There were 40 patients in the cohort to determine objective data during heller myotomy and Dor fundoplication. The DI increased from a median of 0.7 to 3.2 after myotomy and decreased to 2.2 after Dor fundoplication $(\mathrm{p}<0.001)$. The median Eckardt score went down from a median of 4.5 to $0(\mathrm{p}<0.001)$ after surgery.

Conclusion: Our study shows that intraoperative use of EndoFLIP can facilitate diagnosis of achalasia, and used as an adjunct to diagnose achalasia when symptoms are inconsistent. The routine use of EndoFLIP during Heller myotomy and Dor fundoplication provides objective data during the operation in a group of patients with resultant excellent short term outcomes.

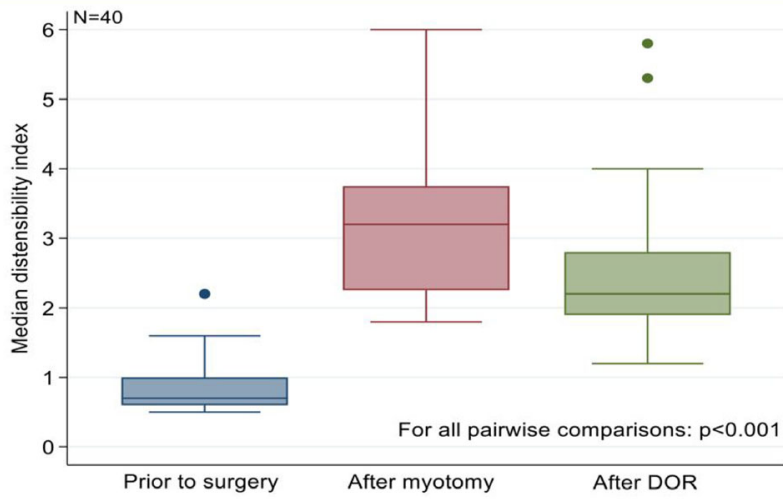

Figure 1. Comparison of distensibility index (DI) prior to surgery, after myotomy and after Dor fundoplication 
S211 Evaluation Of Gastro-Esophageal Reflux In Morbidly Obese
Patients Before And After Laparoscopic Sleeve Gastrectomy Using 24 h Multichannel Intraluminal Impedance pH Monitoring

Gyan Saurabh, MS, FRCS, Glasg; Suruchi Kumari; Manoj Andley, MS, FRCS Glasg; Aparna Agrawal, MD; Lady Hardinge Medical College and Associated Hospitals.

Aims and Objectives: The aim of this prospective observational study was objective analysis of Gastro-esophageal reflux (GER) by $24 \mathrm{~h}$ Multichannel Intraluminal Impedance (MII) pH monitoring, Upper Gastro-intestinal endocscopy ( UGIE) and GERD-Q Questionnaire before and after Laparoscopic Sleeve Gastrectomy (LSG). Methods: A total of 42 morbidly obese patients who underwent LSG were included in the study. Patient were evaluated preoperatively and 3 month postoperatively with UGIE, $24 \mathrm{~h}$ MII pH manometry and GERD-Q Questionnaire. Study was registered in Clinical Trials Registry, India with registration number CTRI/2019/06/019705.

Results: The mean age of our 42 patients ( $73.8 \%$ females) was 38.8 yrs. Mean BMI of patients preoperatively was $43.5 \mathrm{~kg} / \mathrm{m} 2$ which deceased to $32.73 \mathrm{~kg} / \mathrm{m} 2$ (p < 0.001 )postoperatively with \% Excess weight loss (\%EWL) of 25.56 postoperatively. In UGIE, 8 patients $(19.05 \%)$ developed denovo esophagitis, 9 patients $(21.43 \%)$ patients showed worsening of esophagitis, while 3 patients $(7.14 \%)$ showed improvement in esophagitis and 22 patients $(52.38 \%)$ showed no change Overall there is a significant increase in esophagitis ( $\mathrm{p}$ value $=0.014$ )

In $24 \mathrm{~h}$ MII pH monitoring, mean DeMeester score pre-operatively and post operatively were $15.61( \pm 22.35)$ and $19.03( \pm 30.44)$ respectively with the rise being statistically non- significant $(\mathrm{p}$ value $=0.547$ ). Based on DeMeester score $>$ 14.7, preoperatively 12 patients had GER while post-operatively 17 patients had GER ( $\mathrm{p}$ value $=0.251$ ). $24 \mathrm{~h}$ MII pH showed development of denovo GER in 9 patients $(21.43 \%)$. There is significant decrease in mean total acidic reflux (TAR) from $37.26 \pm 34.58$ to $30.12 \pm 30.81(\mathrm{p}$ value $=0.017) 0.27(64.28 \%)$ patients showed decrease in TAR while $14(33.33 \%)$ patients showed increase in TAR. There was an increase in the non-acidic reflux (NAR) from $40.00 \pm 39.56$ to $42.26 \pm 43.74$ $(\mathrm{p}=0.831) 0.17(40.47 \%)$ patients showed increase in NAR. Mean amplitude pressure increased from $45.15 \pm 26.27 \mathrm{mmHg}$ to $58.82 \pm 17.32 \mathrm{mmHg}$ postoperatively, $(\mathrm{p}<0.001)$. Mean LES resting pressure decreased from $27.76 \pm 13.80 \mathrm{mmHg}$ to $25.54 \pm 8.19 \mathrm{mmHg}$ postoperatively $(\mathrm{p}=0.220)$. Mean pre-operative GERD-Q score was $7.31 \pm 1.84$, while mean post-operative GERD-Q score was $6.86 \pm 1.22$, ( $\mathrm{p}$ value $=0.087)$.

Conclusion: Considering the rise in DeMeester score and non acidic reflux after LSG, there need to be careful evaluation and follow up when LSG is contemplated. Long term effect of LSG on GER needs to be further evaluated as many studies have shown biphasic effect.

\section{S212}

Should we perform total omentectomy for advanced gastric cancer?: Reappraisal of the role of partial Omentectomy during laparoscopic gastrectomy for advanced gastric cancer

Sangjun Lee, MD; Yunsuk Suh, MD, PhD; So Hyun Kang, MD; Yongjoon Won; Young Suk Park; Sang-Hoon Ahn; Hyung-Ho Kim; Seoul National University Bundang Hospital.

Objective: In the era of minimally invasive surgery, partial omentectomy (PO) has been widely spread as curative surgical procedure for early gastric cancer. However, scientific evidence of the extent of omentectomy still remains unclear especially for advanced gastric cancer (AGC). The aim of this study is to compare the oncologic efficacy and safety between partial omentectomy (PO) and total omentectomy (TO) during laparoscopic surgery for AGC.

Method: We retrospectively reviewed 672 consecutive patients who underwent laparoscopic gastrectomy for AGC with curative intent between 2014 and 2018 in Seoul National University Bundang Hospital. The patients were divided as PO and TO groups. Surgical outcome and postoperative prognosis was compared after 2:1 propensity score matching including age, sex, BMI, tumor size, $\mathrm{T}$ stage, $\mathrm{N}$ stage, gastrectomy type, and clinically EGC/AGC(T1 vs T2-T4)as covariates.

Result: After matching, there was no significant difference of age, sex, BMI, comorbidity, TNM stages, tumor size, tumor location between PO $(\mathrm{N}=254)$ and TO $(\mathrm{N}=177)$ groups. Operation time was significantly shorter in PO group (195.8 \pm 66.6 vs. $248.1 \pm 68.2 \mathrm{~min}, \mathrm{P}<0.001$ ). Pulmonary complication within postoperative 30 days was significantly lower in $\mathrm{PO}$ group $(3.9 \%$ vs. $9.7 \%, \mathrm{P}=0.035)$. However, there were no difference in other complications $(9.4 \%$ vs. $14.1 \%, \mathrm{P}=0.133)$ and length of stay $(6.9 \pm 4.0$ vs. $7.4 \pm 4.4$ days, $P=0.212)$. 3-year overall survival rate (OS) and recurrence-free survival rate (RFS) was not significantly different between TO and PO groups. ( $83.9 \%$ vs. $81.0 \%, \mathrm{P}=0.854$ for $\mathrm{OS}, 74.2 \%$. Vs. $66.5 \%, \mathrm{P}=$ 0.152 for RFS). Overall recurrence pattern was also not significantly different between two groups $(20.4 \%$ vs. $23.7 \%), \mathrm{P}=0.481)$. In Borrman type IV AGC, RFS for peritoneal seeding was significantly longer in PO than TO (median 43.4 vs. 19.8 months, $\mathrm{P}=0.020$ ), but OS was not different. Cox regression model revealed that TO was the independent risk factor for peritoneal seeding in Borrman type IV AGC $(H R=2.960,95 \%$ C.I $=1.043-8.402, \mathrm{P}=0.041)$.

Conclusion: In laparoscopic gastrectomy for AGC, PO demonstrated significantly shorter operation time, less pulmonary complication, and similar 3-year overall/ recurrence-free survival rate compared with TO. In Borrman type IV AGC, PO provided significantly longer RFS for peritoneal seeding than TO. 
S213

Association of Socioeconomic Status with 30- and 90-Day Readmission Following Open and Laparoscopic Hernia Repair: A National Readmissions Database Analysis

James W Feimster, MD; Brandt D Whitehurst, MD; Adam J Reid, MD; Steve Scaife,

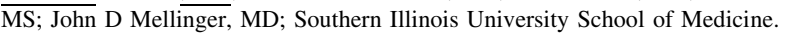

Introduction: Socioeconomic disparities have been associated with outcomes in many medical conditions. The association of socioeconomic status (SES) with readmissions after ventral and inguinal hernia repairs has not been well studied on a national level. This study aims to evaluate the association of SES with readmission as a significant outcome and cost metric in patients undergoing ventral and inguinal hernia repair. We hypothesize that socioeconomic status will be an independent predictor of readmission, and may be the strongest predictor of readmission after these commonly performed operations.

Methods: A retrospective cohort study was performed evaluating patients undergoing ventral hernias and inguinal hernias repair with 1:1 matching using the National Readmissions Database (2016-2017). Patients were selected using ICD-10 codes corresponding to open and laparoscopic ventral and inguinal hernia repair. Both 30- and 90-day readmissions were examined. After matching, a multivariate logistic regression analysis was performed using confounding variables including hospital setting, key comorbidities, urgency of repair, sociodemographic status, and payor. Likelihood of 30- and 90-day readmission was reported in odds ratio form, and P-values were considered significant if less than 0.05 .

Results: Readmission rates were $9.32 \%(19,429 / 208,466)$ and $16.64 \%(25,040 /$ 150,442 ) for $30-\& 90$-day readmissions, respectively. The leading diagnosis at readmission was post-operative infection. Patients with Medicaid and in the lower income quartile were more likely to present in an emergent fashion for hernia repair. After matching and performing a multivariate logistic regression analysis a laparoscopic approach (OR $0.646 \& 0.641$ ), elective admission (OR $0.824 \& 0.779$ ), care in teaching hospitals (OR $0.784 \& 0.798$ ) and socioeconomic status (OR $1.250 \&$ 1.229) were statistically significant independent predictors of readmission at both 30 \& 90-days, respectively.

Conclusion: This analysis of the National Readmissions Database supports the hypothesis that a patient's SES is an independent predictor of readmission at 30- \& 90-days following open and laparoscopic ventral and inguinal hernia repair. However, SES was not the strongest predictor of readmission. Rather, a laparoscopic approach was determined to be the strongest predictor. Patients with a lower SES, as represented by patients with Medicaid or those in the lower income quartile, were more likely to undergo hernia repair in the emergent setting. We find that SES status is independently associated with poorer outcomes in patients undergoing hernia repair. Additional studies are needed to identify modifiable factors, which may include access for elective and laparoscopic repair, in order for surgeons to improve outcomes in this disadvantaged population.

\section{S214}

\section{Overcoming the Learning Curve for Robotic Inguinal Hernia Repair}

Cyrus A Farzaneh; Rene Tellez, MD; Bunchhin Huy, BS; Ashton Christian, MD; Jay Ramsay, BS; Justine Chinn, BS; Brian R Smith, MD; Hubert Kim, MD; Marcel W Hinojosa, MD; University of California, Irvine Medical Center.

Introduction: Robotic inguinal hernia repair is a new surgical approach that has gained popularity for the repair of inguinal hernias. Despite the advantages of robotic surgery, it is not the technique of choice for surgeons, given the time it takes to achieve proficiency. The objective is to investigate the learning curve of a minimally invasive trained surgeon when beginning robotic transabdominal pre-peritoneal (TAPP) hernia repairs.

Methods: We retrospectively identified patients that underwent robotic TAPP hernia repairs between 2012 and 2019 at a single institution. The learning curve was evaluated using the cumulative sum (CUSUM) method.

Results: A total of 258 patients were identified (98.4\% male), of which $124(48.1 \%)$ underwent unilateral and $134(51.9 \%)$ underwent bilateral hernia repair. The CUSUM learning curve model illustrated three unique phases of skill acquisition. The first phase was characterized by initial acclimation, with mean operative times of $78.1 \mathrm{~min}$ and $97.9 \mathrm{~min}$ for unilateral and bilateral cases, respectively. The second phase was exemplified by surgical proficiency, with mean operative times of $61.9 \mathrm{~min}$ and $77.7 \mathrm{~min}$ for unilateral and bilateral cases, respectively. The third phase, occurring after 74 cases for unilateral and after 90 cases for bilateral repairs, represented technical mastery, with more complex cases being tackled. Perioperative outcomes and long-term hernia recurrence were similar for patients in each phase of the learning curve.

Conclusions: Our CUSUM analysis identified three stages of the learning curve for robotic TAPP hernia repairs. Our data demonstrates that proficiency and expertise were achieved within 74 cases for unilateral repairs and within 90 cases for bilateral repairs. Despite learning a new modality, perioperative outcomes and long-term hernia recurrence were not affected by the learning curve. 


\section{S216}

\section{Patient Preference Using a HIPPA Compliant Healthcare Texting App Over Conventional Telecommunication}

Kevin V Benavente, MS ${ }^{1}$; Cory Banaschak, DO $^{2}$; Paul Szotek, $\mathrm{MD}^{3}$; ${ }^{1}$ Marian University College of Osteopathic Medicine; ${ }^{2}$ St. Vincent General Surgery Residency; ${ }^{3}$ Indiana Hernia Center.

The ability to communicate with patients effectively continues to be a major hinderance for physicians and physician offices. However, the advent of numerous technologic advancements and software solutions have offered opportunities to enhance the accessibility of healthcare providers with their patients. We investigated patient preference using a smartphone, HIPPA compliant healthcare texting app, compared to conventional telecommunication with the physician office, in perceived quality of care, as well as surveyed the impact that these interfaces have on preventing unwarranted ER visits. This study utilized a retrospective review from a single private-practice surgeon specializing in hernia repair in Indianapolis, Indiana. Patients with access to a smart phone who received care between July 2017- March 2020 were offered care with the direct-to-provider texting healthcare app, Klara. The Klara patient communication app allows for direct-to-provider text messaging, and was developed in 2016. An anonymous, depersonalized link was distributed to all patients with instructions to complete an online survey, assessing satisfaction using the app compared to their cumulative previous experience calling a physician's office, and whether they preferred using the texting app vs. conventional telecommunication. Patient demographic information was surveyed, and were also asked if they considered presenting to the emergency department due to concerning symptoms. Those who replied yes were then surveyed whether using the app helped avoid an unnecessary ED visit. 90 patients successfully completed the entirety of the survey, median age range 50-60 years old, with the highest category of respondents being between $60-70$ years old. $97 \%$ of respondents confirmed the app was noninferior to conventional telecommunication mediums, with a majority $(75 \%)$ experiencing an relatively improved quality of care. Additionally, $9 \%$ of all respondents had an unnecessary ED visit avoided after consulting their physician through Klara. These results demonstrate that patients find communication with their physician via mobile texting to be superior to the conventional method of phone calls to the physicians office. These results were apparent despite the predominance of older respondents included in this study, which challenges conventional notions that modern technological innovations may be too complicated or inaccessible to this population of patients. However, a discrepancy between those who perceived the quality of care with the app to be comparable or superior to conventional telecommunication (97\%), compared to those who would overall prefer the mobile app over calling the office $(86 \%)$ may signify the momentum of conventional telecommunication making adoption of contemporary technology slower.

\section{S217}

\section{Decision Regret Following Elective Inguinal Hernia Repair}

Ryan Howard, MD; Anne Ehlers, MD, MPH; Lia Delaney, BS; Dana Telem, MD, $\overline{\mathrm{MPH}}$; Laura Mazer, MD, MHPE; University of Michigan.

Introduction: Inguinal hernia repair is an extremely common general surgery operation. Although patients typically report improved quality of life, there are potential complications including chronic pain that might lead patients to regret their decision to undergo surgery. Regret is not commonly included in studies of patientreported outcomes, and the incidence of decision regret among patients undergoing inguinal hernia repair is unknown.

Methods: This cross-sectional study analyzed the Michigan Surgical Quality Collaborative, a statewide registry of patient-reported and clinical outcomes from 73 hospitals. Included patients underwent elective inguinal hernia repair between January 2017-March 2020 and completed validated post-discharge surveys of regret to undergo surgery. Regret was scored on a scale from 1 (absolutely regret surgery) to 5 (absolutely no regret) and dichotomized into "some regret" for scores of 1-4 and "no regret" for a score of 5. 30-day outcomes included complications, emergency department utilization, readmission, and reoperation. Multivariable logistic regression examined the association of regret with age, sex, race, insurance status, ASA, tobacco use, diabetes, admission status, surgical approach, year, and outcomes.

Results: 6156 patients were included. Median follow-up was 55 days. Mean age was $61 \pm 15$ years and $579(9 \%)$ patients were female. $2977(48 \%)$ patients underwent open repair, 2252 (37\%) patients underwent robotic repair, and $927(15 \%)$ underwent laparoscopic repair. Postoperatively, $304(5 \%)$ patients visited an emergency department, $72(1 \%)$ were readmitted, and $63(1 \%)$ underwent reoperation. Overall, $533(9 \%)$ patients reported some regret to undergo surgery. On multivariable logistic regression, regret was associated with visiting an emergency department (OR 2.02, 95\% CI 1.43-2.86), readmission (OR 4.16, 95\% CI 2.32-7.49), female sex (OR 1.42, 95\% CI 1.07-1.88), and tobacco use (OR 1.39, 95\% CI 1.09-1.76). Compared to patients who underwent open repair, patients who underwent minimally invasive repair were less likely to regret surgery (OR $0.77,95 \%$ CI $0.63-0.93$ ). This was driven by less regret among robotic repair (OR $0.70,95 \%$ CI $0.56-0.86$ ) compared to laparoscopic repair (OR 0.94, 95\% CI 0.72-1.22).

Conclusion: Decision regret following inguinal hernia repair affects 1 in 10 patients and is more common than traditional 30-day clinical complications. Visiting an emergency department, being readmitted, female sex, and open approach were the major factors associated with regret after surgery. Robotic repair was associated with lower incidence of regret. Understanding how patient characteristics influence postoperative regret may better enable surgeons to counsel patients and improve their recovery experience. 
S218

\section{Robotic Posterior Plication of Diastasis Recti: Early Experience and Lessons Learned}

Xiaoxi Feng, MD, $\mathrm{MPH}^{1}$; Negin Fadaee, $\mathrm{BS}^{2}$; Shirin Towfigh, $\mathrm{MD}^{2} ;{ }^{1}$ Cedars Sinai Medical Center; ${ }^{2}$ Beverly Hills Hernia Center.

INTRODUCTION: Diastasis recti (DR) is a thinning and widening of the linea alba, often resulting in an abnormal abdominal wall contour. This can lead to core instability, back pain, decreased quality of life and patient dissatisfaction. Though abdominoplasty is the standard of care for this problem, it is often not an option in patients without associated skin laxity. We share our experience with robotic posterior plication for DR in patients who are not candidates for abdominoplasty. METHODS: The robotic approach to DR is transabdominal preperitoneal posterior plication of the rectus fascia. This is performed with 3 arms via left-sided access. Once the peritoneum is taken down, the posterior rectus fascia is plicated with at least two layers of non-absorbable suture. Any associated midline hernia is sutured closed. Mesh may be placed. The peritoneum is then closed. No drains are necessary. Postoperatively, patients are instructed to wear a binder and limit their activities for 6 weeks. Pre-, intra-, and post-operative data was collected on patients who underwent this procedure from 2015-2020.

RESULTS: Over five years, 16 patients (11 male, $69 \%$ ) underwent robotic posterior plication of their DR. Average age was 58 years (range 37-79) with average BMI $30.5 \mathrm{~kg} / \mathrm{m} 2$ (range 22.8-38.6). The average width of the DR was $4.6 \mathrm{~cm}$ (range $2-6 \mathrm{~cm})$. Ten $(63 \%)$ had a concurrent umbilical hernia repair, and $5(31 \%)$ required mesh in addition to the plication. There were no $(0 \%)$ perioperative complications or surgical site occurrences. Median postoperative reduction of abdominal girth was $5 \mathrm{~cm}$ (range 0-7.6), as documented in 6 patients. Average postoperative pain score at 2 weeks followup was $2 / 10$ (median $1 / 10$ ). Two patients (13\%) had early postoperative recurrence of their diastasis at 2 and 4 months; one underwent recurrent DR closure, with mesh.

CONCLUSIONS: In patients who are not candidates for an abdominoplasty, robotic posterior plication is an alternative treatment for DR closure. Most of our patients were males with a small umbilical hernia within their DR. These patients can expect an average $5 \mathrm{~cm}$ reduction in their abdominal girth. Recovery is well tolerated. All patients were highly satisfied with their abdominal contour and results, including those $13 \%$ who had recurrences. To help reduce the risk of recurrence, we have changed our practice to include mesh in patients with wider diastases or with larger abdominal girths.

\section{S219}

\section{REVIEW OF SAGES GERD GUIDELINES AND RECOMMENDATIONS}

Cassandra A Mohr, BS ${ }^{1}$; Hailie N Ciomperlik, BS ${ }^{1}$; Naila Dhanani, MD ${ }^{1}$; Oscar A Olavarria, MD ${ }^{1}$; Craig Hannon, $\mathrm{BS}^{1}$; William W Hope, $\mathrm{MD}^{2}$; Scott J Roth, MD Mike K Liang, MD ${ }^{1}$; Julie L Holihan, $\mathrm{MD}^{1} ;{ }^{1}$ McGovern Medical School at UTHealth; ${ }^{2}$ Department of Surgery, New Hanover Regional Medical Center, Wilmington, North Carolina, USA; ${ }^{3}$ Department of Surgery, Division of General Surgery, University of Kentucky.

INTRODUCTION: SAGES developed evidence-based guidelines for the management of patients with gastroesophageal reflux disease (GERD). The aim of this study is to evaluate guidelines lacking agreement amongst experts (grades B-D).

METHODS AND PRODEDURES: From the SAGES guidelines, six guidelines were chosen for evaluation. A retrospective review of a multicenter database of patients undergoing fundoplication surgery for treatment of GERD between 2015-2020 was performed. Patients that underwent a concurrent gastrectomy or were diagnosed with pre-operative achalasia were excluded. Data were collected on demographics, preoperative, intraoperative, and postoperative variables. Postoperative outcomes were evaluated based on selected SAGES guidelines. Outcomes were assessed using multivariable regression for each guideline.

RESULTS: A total of 444 patients from four institutions underwent surgery for the management of GERD with a median (interquartile range) follow-up of 16 (13) months. Guidelines supported by our data were (1) mesh reinforcement may be beneficial in decreasing the incidence of wrap herniation, (2) robotic assistance is more expensive with similar short-term outcomes to laparoscopic assistance, (3) following laparoscopic antireflux surgery, dysphagia has been reported to significantly improve from preoperative values, and (4) outcomes in older patients are similar to outcomes of younger patients undergoing antireflux surgery. Guidelines that were not supported were (1) the long-term effectiveness of fundoplication in obese individuals (BMI $>30$ ) has been questioned due to higher failure rates and (2) a bougie has been found to be effective.

CONCLUSION: Many SAGES GERD guidelines not receiving Grade A recommendation are supported by large, multicenter database findings. However, further studies at low risk for bias are needed to further refine these guidelines.

\begin{tabular}{|c|c|c|}
\hline SAGES Guidelines under review & Outcome & $\begin{array}{l}\text { Variable Odds Ratio (95\% } \\
\text { Cl), p-value }\end{array}$ \\
\hline $\begin{array}{l}\text { 1. Mesh vs. suture reinforcement: Mesh } \\
\text { reinforcement may be beneficial in decreasing the } \\
\text { incidence of wrap herniation (Grade B). }\end{array}$ & Wrap herniation & $\begin{array}{l}\text { Mesh: } 0.78(0.40-1.51) \text {, } \\
p=0.464\end{array}$ \\
\hline $\begin{array}{l}\text { 2. Robotic vs. laparoscopic assistance: Robotic } \\
\text { assistance is more expensive with similar short- } \\
\text { term outcomes (Grade B). }\end{array}$ & $\begin{array}{l}\text { Dindo clavien } \\
\text { complications }\end{array}$ & $\begin{array}{l}\text { Approach: } \\
\text { Robotic- ref } \\
\text { Laparoscopic: } 1.48 \text { (0.16- } \\
\text { 13.66), } p=0.727 \\
\text { Converted to open: } 8.90 \\
(0.23-333.27), p=0.237\end{array}$ \\
\hline $\begin{array}{l}\text { 3. Pre-operative dysphagia: Following laparoscopic } \\
\text { antireflux surgery, dysphagia has been reported to } \\
\text { significantly improve from preoperative } \\
\text { values (level II-III). }\end{array}$ & Dysphagia & $\begin{array}{l}\text { No dysphagia pre-operative: } \\
14 \% \text { developed post- } \\
\text { operative dysphagia } \\
\text { Dysphagia present pre- } \\
\text { operative: } 84 \% \text { resolved } \\
\text { dysphagia post-operative } \\
p=0.556\end{array}$ \\
\hline $\begin{array}{l}\text { 4. Patient age: }>65 \text { vs. }<65 \mathrm{y} \text { : Outcomes in older } \\
\text { patients are similar to outcomes of younger } \\
\text { patients undergoing antireflux surgery (Grade C). }\end{array}$ & $\begin{array}{l}\text { Dindo clavien } \\
\text { complications }\end{array}$ & $\begin{array}{l}\text { Age } 1.00(0.97-1.03) \text {, } \\
p=0.950\end{array}$ \\
\hline $\begin{array}{l}\text { 5. Patient BMI: The long-term effectiveness of } \\
\text { fundoplication in obese individuals (BMI }>30 \text { ) has } \\
\text { been questioned due to higher failure rates (level } \\
\text { II-III). }\end{array}$ & Wrap herniation & $\begin{array}{l}\text { BMI } 1.00(0.95-1.06) \\
p=0.910\end{array}$ \\
\hline $\begin{array}{l}\text { 6. Bougie efficacy: A } 56 \text { French bougie has been } \\
\text { found to be effective (Grade C). }\end{array}$ & $\begin{array}{l}\text { Post-operative } \\
\text { dysphagia }\end{array}$ & $\begin{array}{l}\text { Bougie } 1.10(0.33-3.26) \\
p=859\end{array}$ \\
\hline
\end{tabular}


S220

\section{Effect of various abdominal wall reconstruction skin closure techniques on surgical site occurrences}

Bryana Baginski ${ }^{1}$; Regan van Metre ${ }^{1}$; Jeremy Warren, $\mathrm{MD}^{2}$; Alex Ewing, $\mathrm{PhD}^{2}$; William Cobb, $\mathrm{MD}^{2}$; Alfredo Carbonell, $\mathrm{DO}^{2} ;{ }^{1}$ University of South Carolina School of Medicine-Greenville; ${ }^{2}$ Department of Surgery, Prisma Health-Upstate.

Introduction: The effect on skin closure technique and its relation to hernia repair technique on surgical site occurrences (SSO) and surgical site infection (SSI) is largely unknown. We hypothesize that layered subcuticular skin closure with cyanoacrylate skin adhesive is protective of surgical site infection compared to standard stapled closure.

Methods: A retrospective review utilizing the Abdominal Core Health Quality Collaborative (ACHQC) database of all patients at Prisma Health - Upstate. All patients with open abdominal wall reconstruction (AWR) of midline incisional hernia defects with retromuscular polypropylene mesh placement from January 2013-February 2020 were included. Patient demographics, comorbidities, type of hernia repair with mesh location, method of skin closure, and SSOs were collected. Skin closure method was divided into two groups, reflecting a temporal change in practice: staples (historical control group) versus subcuticular suture with cyanoacrylate skin adhesive (study group). Primary endpoint was SSI and SSO. Secondary endpoints were SSO or SSI requiring procedural intervention (SSOPI / SSIPI). Standard statistical methods were utilized.

Results: A total of 834 patients were analyzed, with 263 treated with stapled skin closure, 571 with subcuticular and adhesive closure. On univariate analysis, incidence of SSI was significantly lower in the study group ( 11.8 vs $6.8 \% ; \mathrm{p}=0.002)$, as was the need for SSIPI ( 11.8 vs $6.7 \%$; $p=0.015)$. Rate of SSO was not significantly different between groups ( $28.1 \mathrm{vs} 27.2 \%)$, but the rate of SSO requiring intervention was lower in the study group (14.1 vs $9.3 \%$; $p=0.045)$.

Conclusions: Layered skin closure technique, including subcuticular closure and adhesive, may reduce the risk of surgical site infection after open AWR. A prospective randomized trial is planned to confirm these findings.

\section{S221}

Variation in Technique for Ventral and Incisional Hernia Repair Across a Statewide Collaborative

Anne P Ehlers, MD, MPH; Ryan Howard, MD; Laura M Mazer, MD, MHPE; Lia Delaney; Quinton Solano; Michael J Englesbe, MD; Justin B Dimick, MD, MPH; Jennifer F Waljee, MD, MPH, MS; Dana A Telem, MD, MPH; University of Michigan.

Introduction: While wide variation in technique is anecdotally accepted for ventral and incisional hernia repair (VIHR), no study to date has characterized this variation at a population-level. Understanding variation in care is the first step in designing targeted interventions to improve outcomes. Within this context, we sought to characterize practice patterns using the first and only clinically-nuanced US hernia registry to inform future quality improvement efforts.

Methods and Procedures: Adult patients undergoing elective VIHR from January 1-October 7, 2020 in the Michigan Surgical Quality Collaborative (MSQC), a statewide multi-payer registry that includes 73 hospitals with a goal of using data to improve patient outcomes, were included in this study. In January 2020, this registry was enhanced to include clinically-nuanced variables pertaining to hernia characteristics including hernia size, planned approach (e.g. open, laparoscopic, robotic), use of mesh, and mesh location. Patients were stratified by hernia width $(\leq 2 \mathrm{~cm}$, $2-5 \mathrm{~cm}, 5-10 \mathrm{~cm}, \geq 10 \mathrm{~cm}$ ).

Results: Among 526 patients, $143(27 \%)$ had a hernia $\leq 2 \mathrm{~cm} ; 242(46 \%) 2-5 \mathrm{~cm}$; $96(18 \%) 5-10 \mathrm{~cm}$; and $45(9 \%) \geq 10 \mathrm{~cm}$. Open surgery was more common for smaller hernias ( $80 \%$ for hernia $\leq 2 \mathrm{~cm}$ vs $58 \%$ for hernia $\geq 10 \mathrm{~cm}, \mathrm{p}<0.001$ ).

When a minimally invasive approach was selected, the robotic approach was more common in all cohorts except hernia $\leq 2 \mathrm{~cm}$. Mesh was used in $78 \%$ of patients and was used almost uniformly for larger hernias $(96 \%$ for hernia $>10 \mathrm{~cm})$ while use in small hernias was variable ( $53 \%$ for hernia $\leq 2 \mathrm{~cm}, \mathrm{p}<0.001)$. Myofascial release was specified in $<1 \%$ of hernias $\leq 2 \mathrm{~cm}$ but in $38 \%$ of hernias $\geq 10 \mathrm{~cm}(\mathrm{p}<0.001)$. Complete data is demonstrated in the Table.

Conclusions: This is the first study to characterize population-level variability in approach to hernias of different sizes. Wide variability was noted with lack of consensus about the best technique, particularly for small hernias. Future work will correlate VIHR technique to patient outcomes to inform best practices and promote right-sized interventions for this common and morbid disease.

\begin{tabular}{|c|c|c|c|c|c|}
\hline & $\begin{array}{c}\leq 2 \mathrm{~cm} \\
(\mathrm{~N}=143)\end{array}$ & $\begin{array}{c}2-5 \mathrm{~cm} \\
(\mathrm{~N}=242)\end{array}$ & $\begin{array}{l}5-10 \mathrm{~cm} \\
(\mathrm{~N}=96)\end{array}$ & $\begin{array}{l}\geq 10 \mathrm{~cm} \\
(\mathrm{~N}=45)\end{array}$ & p-value \\
\hline \multicolumn{6}{|l|}{ Approach } \\
\hline Open & $114(80)$ & $149(62)$ & $60(63)$ & $26(58)$ & \multirow{3}{*}{0.001} \\
\hline Laparoscopic & $8(6)$ & $57(24)$ & $20(21)$ & $14(31)$ & \\
\hline Robotic & $20(14)$ & $30(12)$ & $15(16)$ & $5(11)$ & \\
\hline Mesh used & $76(53)$ & $209(86)$ & $80(83)$ & $43(96)$ & $<0.001$ \\
\hline \multicolumn{6}{|l|}{ Mesh location } \\
\hline Onlav & $26(43)$ & $52(31)$ & $23(34)$ & $8(24)$ & \multirow{3}{*}{0.500} \\
\hline Inlay & $4(7)$ & $11(7)$ & $6(9)$ & $4(12)$ & \\
\hline Sublay & $30(50)$ & $103(62)$ & $39(57)$ & $21(64)$ & \\
\hline \multicolumn{6}{|l|}{ Sublay location } \\
\hline Retromuscular. & $5(17)$ & $13(13)$ & $16(42)$ & $7(33)$ & \multirow{3}{*}{0.001} \\
\hline Preperitoneal & $14(48)$ & $46(46)$ & $8(21)$ & $3(14)$ & \\
\hline Intraperitoneal & $10(34)$ & $42(42)$ & $14(37)$ & $11(52)$ & \\
\hline \multicolumn{6}{|l|}{ Type of mesh } \\
\hline Synthetic (Non-Abs) & $44(67)$ & $107(58)$ & $22(31)$ & $8(21)$ & \multirow{5}{*}{$<0.001$} \\
\hline Synthetic (Abs) & $14(21)$ & $55(30)$ & $38(54)$ & $25(64)$ & \\
\hline Biosynthetic & $5(8)$ & $14(8)$ & $4(6)$ & $3(8)$ & \\
\hline Biological & $1(2)$ & $5(3)$ & $5(7)$ & $2(5)$ & \\
\hline Other & $1(2)$ & $3(2)$ & $1(1)$ & $0(0)$ & \\
\hline Myofascial Release & $1(<1)$ & $9(4)$ & $11(11)$ & $17(38)$ & $<0.001$ \\
\hline
\end{tabular}


S222

\section{COMPARATIVE ENVIRONMENTAL EFFECTS AND DISABILITY-ADJUSTED LIFE-YEAR IMPACTS OF ROBOTIC AND OPEN VENTRAL HERNIA REPAIR}

Joseph M Blankush, $\mathrm{MD}^{1}$; Andrea Gardiner, $\mathrm{PhD}^{2}$; Vikram Tiwari, $\mathrm{MBA}^{3}$; Marcus Jordan ${ }^{1} ;$ Hiba Baroud, $\mathrm{Ph}^{2} ;$ David Koester, $\mathrm{PhD}^{2}$; Douglas Adams, $\mathrm{PhD}^{2}$; Joseph $\mathrm{R}$ Broucek, $\mathrm{MD}^{1} ;{ }^{1}$ Vanderbilt University Medical Center; ${ }^{2}$ Vanderbilt University School of Engineering; ${ }^{3}$ Owen School of Management.

OBJECTIVE: By 2030, 250,000 deaths will be attributable annually to global climate change. The global healthcare sector accounts for $4.4 \%$ of global $\mathrm{CO} 2$ emissions, and as a nation would be the fifth largest emitter. The US healthcare sector's environmental impact causes 614,000 years of healthy life lost annually, or disability-adjusted life-years (DALYs). Conversely, 400,000 ventral hernia repairs (VHR) are completed annually in the US, and previous studies have suggested that up to 9 DALYs are averted with a single hernia repair. Little research exists, however, identifying specific environmental impacts of common general surgery procedures such as VHR and the associated downstream impacts of procedureassociated products and processes.

METHODS: 25 cases each of robotic (rVHR) and open ventral hernia repair (oVHR) inclusive in each case of myofascial flap creation and mesh implantation were inventoried and analyzed using both Life-Cycle Assessment (LCA) and Economic Input-Output Life-Cycle Assessment methodologies (EIO-LCA, database: www.eio-lca.net). Point-of-care cost and associated economic sectors for surgical instruments, medical supplies, intra-operative energy use, device maintenance, and waste processing/disposal were included in the analysis. LCA analysis was carried out using SimaPro software (Pré Sustainability, Amersfoort, Netherlands). TRACI 2.1 and Impact $2002+$ impact assessment databases were used to characterize and quantify cradle to grave environmental impacts of each product or process.

RESULTS: rVHR demonstrates significantly larger impacts across all measured environmental impact categories (Fig. 1). On average, each rVHR generates 144\% (609 kg CO2eq) more greenhouse gas (GHG) emissions over oVHR. GHG emissions associated with supply chain transportation account for $26 \%$ and $24 \%$ of rVHR- and oVHR-associated emissions respectively. Energy-associated extraction, refinery and generation accounts for 39\% of rVHR CO2eq emissions and $45 \%$ of oVHR emissions. Embodied energy attributable to rVHR is $143 \%$ greater - an additional 1.4 barrel of oil equivalents per procedure. rVHR resulted in $18 \%$ additional municipal solid waste per case. Downstream environmental effects cause an incremental $1.9 \times 10-3$ DALYs per rVHR, $4.7 \times 10-3$ DALYs overall per procedure.

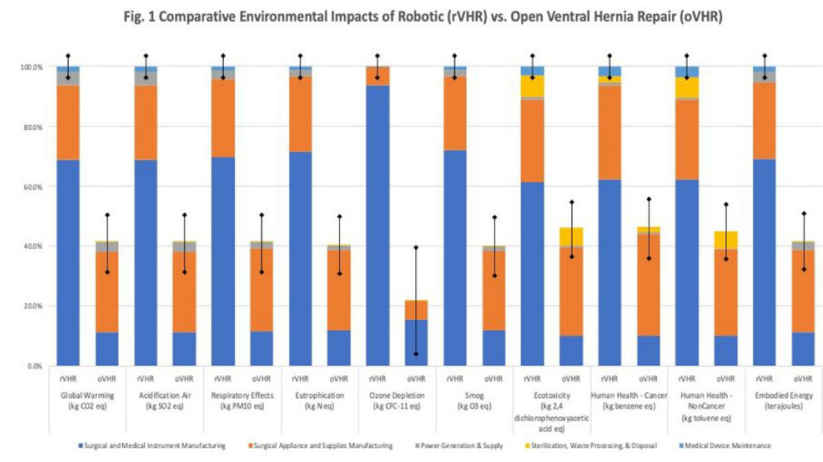

CONCLUSION: rVHR produces significantly higher environmental impacts compared to oVHR. Data to date suggests that the net DALY avoidance per VHR patient far outweighs the public health impact of each procedure's environmental impact, but the effect on population health is not insignificant. Surgeons, administrators, and policymakers should partner with suppliers to explore materials and process alternatives, especially in the sector's supply chain and energy sources, to avoid or mitigate climate-related social and public health effects during widespread adoption of robotic surgery.

\section{S223}

\section{DECONSTRUCTING THE OPERATIVE DURATION OF ROBOTIC PANCREATICODUODENECTOMY}

Alexander Rosemurgy, MD, FACS; Abigail Espeut, MS, MBA; Padma Vasanthakumar; Timothy Bourdeau, BS; Valerie Przetocki, BS; Iswanto Sucandy, MD, FACS; Sharona Ross, MD, FACS; AdventHealth Tampa.

Introduction: Despite the numerous benefits of the robotic platform, robotic operations take longer than 'open' operations. With a goal of decreasing the duration of robotic pancreaticoduodenectomy, this study was undertaken to determine to what degree each operative step contributes to operative duration of robotic pancreaticoduodenectomy.

Method: With IRB approval, the operative duration of 74 robotic pancreaticoduodenectomies were deconstructed; for 44 operations the duration of 19 key steps were recorded (Figure). The beginning of each operative step was called out by the surgeons and recorded by circulating nurses. Data are presented as median(mean \pm $\mathrm{SD})$. For each operative step regression analysis determined the impact of our experience.

Results: Patients were $70(69 \pm 12.3)$ years-old; $50 \%$ were men. Length of stay was $4(75.1)$ days. 65 ( $88 \%)$ had neoplastic disease; R0 resections were achieved in 62 $(96 \%)$ of 65 patients. Time between patient in-and-out of operating room was $466(478 \pm 89.1)$ minutes. Total operative duration was $397(414 \pm 89.2)$ minutes. Total console time was $293(287 \pm 58.4)$ minutes. The duration of each step did not decrease with experience.

Conclusion: A third of operative duration was spent off the console. The three longest steps of the operations were the removal of the specimen off the portal vein/ SMV/SMA, division of CHD, and cholecystectomy; construction of the duodenojejunostomy; and closure. Over a third of the steps required more than $20 \mathrm{~min}$ each to complete. Robotic Surgery is a burgeoning approach that we believe is associated with a shorter LOS, decreased EBL, fewer complications, and longer survival. Further salutary benefit may be gained by decreasing operative duration. Operative duration is an important metric that needs to be addressed by all stakeholders (e.g., surgeons, anesthesiologists, nurses, pathologists). Operative duration needs to be addressed through a team approach to reach optimal efficiency and salutary benefit.

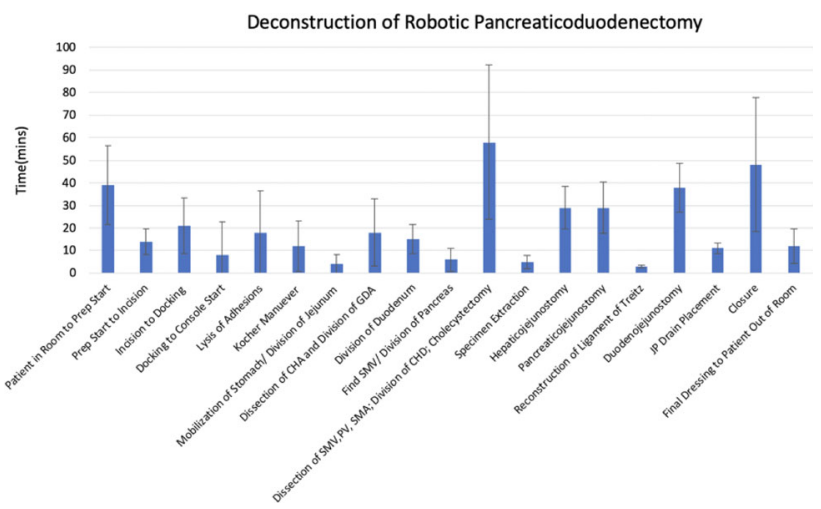


S224

\section{What a Relief: Decreased Urinary Retention with ERAS Protocol for Minimally Invasive Inguinal Hernia Repair}

Ryan C Broderick, MD; Rachel R Blitzer, MD; Jonathan Z Li, MD; Alice Race, MD; Gene Yang, MD; Bryan J Sandler, MD; Santiago Horgan, MD; UC San Diego.

Background: Minimally invasive inguinal hernia repair (IHR) is one of the most common general surgery procedures. Though these repairs tend to be low-risk outpatient surgeries, potential side effects include post-operative urinary retention (POUR), with $1-20 \%$ of patients requiring indwelling catheter placement. POUR is known to be influenced by time under anesthesia, perioperative fluid volume, and opiate administration. An Enhanced Recovery After Surgery (ERAS) protocol was developed at our institution for outpatient inguinal hernia repairs, which aims to mitigate contributors to delayed surgical recovery; implementation began in early 2018, with an emphasis on reducing postoperative narcotic prescriptions. This study assesses the impact of ERAS measures on urinary retention and uncontrolled pain after minimally invasive inguinal hernia repair.

Methods: A retrospective review of a prospectively maintained database identified patients that underwent minimally invasive inguinal hernia repair at a single institution. Patient demographic data including age, sex, and previous surgical history were recorded. Exclusion criteria were open or concurrent procedures. Outcomes studied include complications, postoperative pain, and urinary retention. Patients whose surgeries occurred after 2018 were in the ERAS cohort; a preference for nonnarcotic post-operative treatment was also employed. Patients who had uncontrolled post-operative pain were defined as those who required additional narcotics, admission for pain, or who returned to the emergency department (ER) for treatment of pain. POUR was defined as patients requiring an indwelling urethral catheter at discharge or who reported to the ER for treatment of urinary retention.

Results: Between January 2008 and August 2020, 1032 patients who underwen minimally invasive inguinal hernia repair were identified. The majority $(90.8 \%)$ of these procedures were laparoscopic, the rest robotic. Average patient age was 57.2 years and $93.9 \%$ were male. Patients receiving care under ERAS protocol were significantly less likely to experience POUR when compared to their prior counterparts $(0.10 \%$ vs. $4.3 \% \mathrm{p}=0.014)$; they were also less likely to experience uncontrolled pain $(0.2 \%$ vs. $2.9 \% \mathrm{p}=0.003)$. Of note, the subgroup of patients on ERAS protocol and were not prescribed post-op narcotics $(n=116)$ had $0 \%$ POUR. Patients with POUR were significantly older than those without $(67.9$ vs. 56.9 years, $\mathrm{p}<0.0001$ ). Within the ERAS group, $71.8 \%$ of patients received postoperative narcotics while $100 \%$ of the pre-ERAS group were prescribed opiate pain medications.

Conclusion: Outpatient ERAS protocols and limitation of post-op narcotic prescriptions significantly decrease the risk of urinary retention for minimally invasive inguinal hernia repair.

Figure 1: Postoperative pain and Urinary Retention in Minimally Invasive Hernia Repair

\begin{tabular}{|l|l|l|l|}
\hline & $\begin{array}{l}\text { Narcotics } \\
(n=916)\end{array}$ & $\begin{array}{l}\text { No Narcotics } \\
(n=116)\end{array}$ & p-value \\
\hline Postoperative pain & $21(2.29 \%)$ & $1(0.86 \%)$ & 0.499 \\
\hline Urinary retention & $29(3.17 \%)$ & 0 & 0.066 \\
\hline & & & \\
\hline & $\begin{array}{l}\text { No ERAS } \\
(n=620)\end{array}$ & $\begin{array}{l}\text { ERAS } \\
(n=412)\end{array}$ & \\
\hline Post Op Pain & $19(2.9 \%)$ & $3(0.2 \%)$ & 0.014 \\
\hline Urinary Retention & $25(4.3 \%)$ & $4(0.10 \%)$ & 0.0033 \\
\hline Postoperative Narcotics & $620(100 \%)$ & $296(71.8 \%)$ & $<0.001$ \\
\hline & & & \\
\hline
\end{tabular}

\section{S227}

Minimally invasive versus open distal pancreatectomy:

Dylan M Russell, MD ${ }^{1}$; Alexandra Adams, MD $^{2}$; Timothy Vreeland, MD $^{2}$; Daniel Nelson, $\mathrm{MD}^{2}$; Christopher Yheulon, $\mathrm{MD}^{\mathrm{I}}$; ${ }^{\mathrm{I}}$ Tripler Army Medical Center; ${ }^{2}$ Brooke Army Medical Center.

Background: Minimally invasive distal pancreatectomy (MIDP) is gaining popularity due to improved perioperative outcomes over open distal pancreatectomy (ODP). However, patient selection often differs between approaches. The purpose of this study is to compare outcomes of MIDP and ODP using propensity-score matched patients within a nationwide cohort.

Methods: The American College of Surgeons' National Quality Improvement Program (ACS-NSQIP) (2005-2018) was used to evaluate incidence of post-operative pancreatic fistula (POPF) as well as 30-day composite major morbidity for patients undergoing MIDP vs ODP. Matching was performed for demographic characteristics, pre-operative risk factors, and benign versus malignant pathology using a genetic matching algorithm implemented via the MatchIt package. Unadjusted and adjusted odds ratio estimates for binomial outcomes with $95 \%$ confidence intervals were generated via weighted multiple logistic regression.

Results: A total of 3940 patients underwent distal pancreatectomy (1978 MIDP 1962 ODP). Prior to matching, the MIDP patients had significantly higher body mass index, lower ASA class, and less pre-operative weight loss whereas ODP patients had significantly higher rates of malignant pathology and receipt of chemotherapy and radiation. After matching, 2985 patients were included (1978 MIDP, 1007 ODP). The rates $(8.65 \%$ MIDP ys. $9.76 \%$ ODP, $p=0.37)$ and odds of major morbidity $(0.88[0.66,1.18], \mathrm{p}=0.386)$ were similar between groups. The MIDP group was found to have significantly decreased length of stay (5.6 vs. 7 days, $p=<$ $0.001)$, but greater rates $(12.54 \%$ MIDP vs. $9.35 \%$ ODP, $\mathrm{p}=0.02)$ and odds $(1.40$ $[1.06,1.85], \mathrm{p}=0.018)$ of post-operative fistula.

Conclusions: When matched for baseline patient characteristics, MIDP was associated with shorter length of hospitalization with similar rates of composite major morbidity compared to ODP. Despite quicker recovery, MIDP was associated with $40 \%$ increased risk of clinically significant POPF. Further studies are needed to investigate this difference in POPF rate, and how to optimize MIDP surgical technique to reduce the risk. 


\section{A SURVEY OF PATIENT AND SURGEON PREFERENCE FOR EARLY ILEOSTOMY CLOSURE FOLLOWING RESTORATIVE PROCTECTOMY AND PROTECTIVE ILEOSTOMY FOR RECTAL CANCER - WHY AREN'T WE DOING IT?}

Natasha Caminsky ${ }^{1}$; Jeongyoon Moon ${ }^{1}$; Fateme Rajabiyazdi ${ }^{2}$; Sami A. Chadi ${ }^{3}$; Karim Alavi ${ }^{4}$; Ian M. Paquette ${ }^{5}$; Tony MacLean ${ }^{6}$; Steven D. Wexner ${ }^{7}$; A. Sender Liberman $^{2}$; Scott Steele ${ }^{8}$; Jason Park'; Sunil Patel ${ }^{10}$; Liliana G. Bordeianou ${ }^{11}$; Rebecca C. Auer ${ }^{12}$; Patricia Sylla ${ }^{13}$; Nancy Morin ${ }^{1}$; Marylise Boutros ${ }^{1} ;{ }^{1}$ Jewish General Hospital; ${ }^{2}$ McGill University Health Center; ${ }^{3}$ University Health Network and Princess Margaret Hospital; ${ }^{4}$ University of Massachussetts; ${ }^{5}$ University of Cincinnatti; ${ }^{6}$ Foothills Medical Centre, University of Calgary; ${ }^{7}$ Cleveland Clinic Florida; ${ }^{8}$ Cleveland Clinic; ${ }^{9}$ St. Boniface General Hospital, University of Manitoba; 10Queens University; ${ }^{11}$ Massachusetts General Hospital and Harvard Medical School; ${ }^{12}$ The Ottawa Hospital; ${ }^{13}$ Icahn School of Medicine at Mount Sinai.

INTRODUCTION-Early ileostomy closure (EIC), reversal of a defunctioning stoma $\leq 2$ weeks from creation at the index surgery, is a relatively new practice that has been shown to be safe, feasible, and cost-effective in Europe. However, to our knowledge, this is not routine practice, nor has it been studied, in North America. The objective of this study was to assess patient and surgeon opinions about EIC and assess interest for future research and implementation.

METHODS - After institutional ethics review board approval, we conducted a mixed-methods, cross-sectional study of patients and surgeons. Rectal cancer survivors from a tertiary-care practice in Canada were considered for inclusion if they had undergone restorative proctectomy with diverting loop ileostomy and subsequent reversal within the last 4 years. North American surgeons with high rectal cancer volumes ( $>20$ cases/year) were included. Structured (for patients) and semi-structured (for surgeons) interviews were performed by telephone or Zoom-Pro. Grounded theory was used to thematically analyze the transcribed interviews using MAXQDA.

RESULTS - A total of 24 patients were interviewed. Mean age was $65 \pm 13$ years (54\% female). Patients had their stomas for a median of 7.2 months (IQR 4.6-9.9) and found it difficult/very difficult to live with their stoma in $46 \%(\mathrm{~N}=11)$ of cases. The most important advantages of EIC perceived by patients were an improvement in quality of life and quicker return to normal function, whereas the most important disadvantage was that two operations in two weeks would be too taxing on the body. The majority of patients $(63 \%, \mathrm{~N}=15)$ would have chosen EIC had it been offered to them. Surgeon interviews $(N=13)$ revealed 4 overarching themes: (1) many barriers to implementing EIC exist including logistical difficulty, concerns for patient outcomes, and resistance to change; (2) only motivated patients with an uncomplicated peri-operative course should be eligible for EIC; (3) successful implementation will require a strategic approach including multidisciplinary and institutional buy-in; (4) there are many benefits to EIC including avoiding stoma-related complications and reducing financial burden to patients and the healthcare system. The majority of surgeons $(\mathrm{N}=11,85 \%)$ said they would definitely want to participate in a randomized controlled trial.

CONCLUSIONS - Although EIC has been proven safe, it has not been implemented in North America because of many logistical challenges. Both patients and surgeons are interested in further exploring this approach and believe it warrants a North American randomized trial to motivate a change in practice.

\section{OPIOID USE DISORDER ASSOCIATED WITH INCREASED ANASTOMOTIC LEAK AND MAJOR COMPLICATIONS AFTER COLORECTAL SURGERY}

Jessica Holland; Jeongyoon Moon; Daniel Marinescu; Nancy Morin; Gabriela Ghitulescu; Allison J. Pang; Carol-Ann Vasilevsky; Marylise Boutros; Jewish General Hospital.

Introduction: Opioid use disorders (OUD) have been associated with an increased risk of complications, length of stay and cost in many surgical procedures. This study aims to determine their impact on the outcomes of colorectal resections.

Methods \& Procedure: Using the National Inpatient Sample database, all admissions for colorectal resections between 2000-2016 were identified using ICD-9/10 codes. Patients with a history of OUD were identified using ICD-9/10 codes. The outcomes of interest were mortality, post-operative complications, length of stay and cost of admission. Patient and disease factors associated with OUD were studied using multivariable logistic regression. Multivariable logistic and linear regression models were used to study the association of OUD and the outcomes of interest. Covariates for the models were chosen a priori and included age, sex, steroid use, smoking, indication for surgery, type of resection, select comorbidities, select socioeconomic factors as well as others factor judged to be potential clinically relevant.

Results/Outcomes: Of 1,599,767 admissions for colorectal resections, 5,953 $(0.37 \%)$ patients had an OUD. Patients with OUDs were younger $(56.0+16.1 \mathrm{vs}$ $62.2+17.1, \mathrm{p}<0.01)$, more likely to be smokers $(27.6 \%$ vs. $21.6 \%, \mathrm{p}<0.001)$, overweight or obese $(10.9 \%$ vs. $8.6 \%, \mathrm{p}<0.001)$ and less likely to be undergoing elective surgery $(33.1 \%$ vs. $49.1 \%, \mathrm{p}<0.001)$. These patients also had a high proportion of mental health disorders $(34.1 \%$ vs $11.6 \%, \mathrm{p}<0.001)$ and other substance use $(11.9 \%$ vs $1.6 \%, \mathrm{p}<0.001)$. On crude analysis, OUD was associated with increased overall complication $(34.0 \%$ vs $48.3 \%, p<0.0001)$ and anastomotic leak rates $(5.4 \%$ vs $8.28 \%, \mathrm{p}<0.001)$. OUD was also associated with a longer length of stay, $(8.83+11.5$ vs. $10.74+13.6$ days, $p<0.01)$ and increased cost of admission $(\$ 66,494+114,444$ vs. $\$ 72,258+195,513, \mathrm{p}<0.01)$. On multivariate logistic regression, OUD remained significantly associated with overall operative complications (OR 1.54; 95\% CI 1.34, 1.77) and anastomotic leak (OR 1.29; 95\% CI 1.08, 1.55). However, OUD did not have a significant impact on mortality (OR $0.90,95 \%$ CI $0.64,1.25)$. On multivariable linear regression, OUD remained associated with an increased hospitalization cost of $\$ 26,365.59$ (95\% CI $26365.50,26,365.50)$ and increased length of stay of 2.60 days (95\% CI 2.54, 2.65).

Conclusions: OUD represents a significant predictor of overall complications, anastomotic leak, increased cost, and length of stay in major colorectal surgery. This patient group should receive dedicated pre-operative optimization and careful postoperative monitoring in order to improve their outcomes. 


\section{S233}

\section{GASTRIC ISCHEMIC CONDITIONING REDUCES ANASTOMOTIC COMPLICATIONS IN PATIENTS UNDERGOING ESOPHAGECTOMY: A SYSTEMATIC REVIEW AND META-ANALYSIS}

Uzair Jogiat, MD; Warren Sun, VMD; Jerry T Dang, MD; Valentin Mocanu, MD; Janice Y Kung, BCom, MLIS; Shahzeer Karmali, MD, MPH; Simon R Turner, MD, MEd; Noah J Switzer, MD, MPH; University of Alberta.

Introduction: Esophageal resection with gastric conduit reconstruction remains the mainstay of surgical management for esophageal cancer, yet it is associated with high rates of complications such as anastomotic leak, gastric conduit ischemia and anastomotic stricture. Gastric ischemic conditioning (GIC) is a novel strategy to promote neovascularization of the gastric conduit in order to reduce the risk of anastomotic complications. We aimed to systematically review the literature to determine the impact of GIC on outcomes following esophagectomy.

Methods and Procedures: A systematic comprehensive search in MEDLINE, EMBASE, Scopus, Web of Science and Cochrane Library was performed on February 5,2020 , by a university librarian. Two authors independently screened fulltext articles. Inclusion criteria included human participants undergoing esophagectomy with gastric conduit reconstruction, age $\geq 18, \mathrm{~N} \geq 5$, GIC performed prior to esophagectomy. Our primary outcomes of interest were anastomotic leaks, gastric conduit ischemia, and anastomotic strictures. Our secondary outcome included mortality. A meta-analysis using RevMan 5.4.1 was performed using a MantelHaenszel, fixed-effects model.

Results: A total of 1712 preliminary studies were identified with 1071 abstracts screened after removal of duplicates and 23 studies (15 retrospective chart reviews, 2 retrospective studies, 2 prospective studies, and 2 non-randomized trials) included for final review. A total of 2203 patients, the majority of which were male ( $\mathrm{n}=1515$, $69.8 \%$ ) with a mean age of 63 years \pm 7.1 were included. GIC was performed in $1178(53.5 \%)$ patients with a mean time from GIC to surgery of 28 days \pm 37.3 . Surgical complications included 47 deaths (2.2\% GIC vs $2.1 \%$ control), 215 anastomotic leaks ( $7.1 \%$ GIC vs $13.1 \%$ control), 30 ischemic conduits $(0.9 \%$ GIC vs $1.9 \%$ control), and 212 anastomotic strictures (3.0\% GIC vs $17.7 \%$ control). Metaanalysis revealed significant improvements for gastric conduit ischemia (OR 0.48; 95\% CI: 0.29-0.80; $\mathrm{p}=0.005$ ), anastomotic leaks (OR 0.67; 95\% CI 0.46-0.97; $\mathrm{p}=$ $0.03)$, and anastomotic strictures (OR $0.48 ; 95 \%$ CI $0.29-0.80 ; \mathrm{p}=0.005)$ in patients undergoing preoperative GIC. Trends towards improved mortality (OR 0.54; $95 \%$ CI: $0.29-1.02, \mathrm{p}=0.06$ ) were also observed though not achieving statistical significance.
Conclusion: GIC prior to esophagectomy is associated with significant improvements in conduit ischemia, anastomotic leaks, and anastomotic strictures. GIC is promising technique that may improve outcomes and decrease surgical morbidity in patients undergoing esophagectomy. Prospective data in the form of a randomized control trial evaluating the aforementioned outcomes is needed before definitive conclusions can be drawn.

A

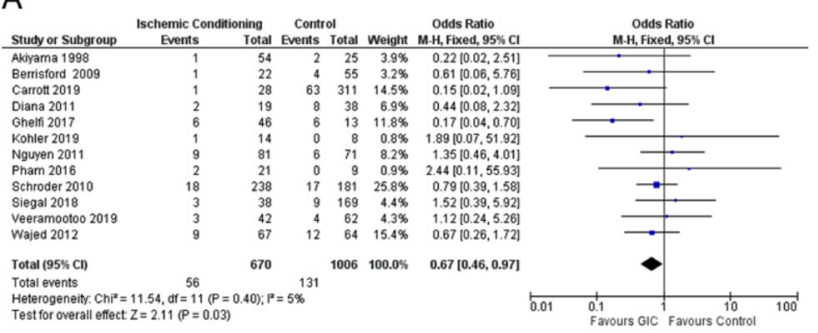

B

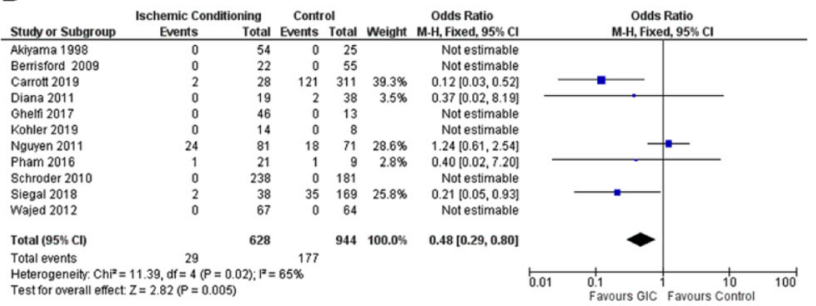

Figure 1. Preoperative GIC via laparoscopy or embolization versus no GIC in Patients undergoing Esophagectomy.

Panel A: Incidence of post-operative anastomotic leaks amongst patients undergoing gastric ischemic conditioning versus controls.

Panel B: Incidence of post-operative anastomotic strictures amongst patients undergoing gastric ischemic conditioning versus controls.

Publisher's Note Springer Nature remains neutral with regard to jurisdictional claims in published maps and institutional affiliations. 